\title{
Flows, morphology, and memory: study of a living network
}

\author{
Dissertation for \\ the award of the degree \\ "DOCTOR RERUM NATURALIUM" \\ of the \\ GeOrg-August-Univeristät Göttingen \\ within the doctoral programme \\ Physics of Biological and Complex Systems \\ of the \\ Göttingen Graduate School of Neurosciences, Biophysics, and Molecular \\ BIOSCIENCES (GGNB) \\ of the \\ Georg-August University School of Sciences (gauss) \\ submitted by \\ Mirna Elizabeta Kramar \\ from Zabok, Croatia
}

Göttingen, 2020 


\section{Thesis Advisory Committee}

\section{Prof. Dr. Karen Alim}

Biological Physics and Morphogenesis

Max Planck Institute for Dynamics and Self-Organization \& Technische Universität München

\section{Dr. Florian Rehfeldt}

Cell and Matrix Mechanics

$3^{\text {rd }}$ Institute of Physics at the Georg-August-University Göttingen \& Universität Bayreuth

\section{Prof. Dr. Fred Wolf}

Theorectical Neurophysics

Max Planck Institute for Dynamics und Self-Organization

Prof. Dr. Helmut Grubmüller

Theoretical and Computational Biophysics

Max Planck Institute for Biophysical Chemistry

\section{EXAMINATION BOARD}

Prof. Dr. Karen Alim (Referee)

Biological Physics and Morphogenesis

Max Planck Institute for Dynamics and Self-Organization \& Technische Universität München

\section{Dr. Florian Rehfeldt (Referee)}

Cell and Matrix Mechanics

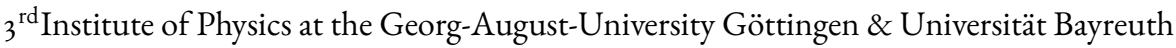

\section{Prof. Dr. Fred Wolf}

Theorectical Neurophysics

Max Planck Institute for Dynamics und Self-Organization

Prof. Dr. Helmut Grubmüller

Theoretical and Computational Biophysics

Max Planck Institute for Biophysical Chemistry

Prof. Dr. Andreas Dillmann

Institute of Aerodynamics and Flow Technology

German Aerospace Center

Prof. Dr. Andreas Janshoff

Biophysikalische Chemie

Institut für Physikalische Chemie 


\section{Abstract}

The complex behaviour of the slime mould Physarum polycephalum, a simple eukaryote, has been puzzling researchers since its discovery. The giant unicellular, but multinucleate organism is highly successful at tackling complex environments, despite being a very simple life form. The organism forages for nutrients and flees from threat by reorganizing its networklike body made of actomyosin-lined tubes. The tubes undergo periodic contractions, causing a shuttle flow of the cytoplasm inside the tubes which in turn transports nutrients, signals and redistributes body mass. Often termed intelligent, the organism displays behaviours usually found in higher species with a nervous system.

In this thesis, we aim to uncover the governing principles behind several phenomena from P. polycephalum's abundant repertoire of behaviours. First, we delve into the memory encoding abilities by studying how the network imprints the location of a nutrient source. Using theoretical and experimental methods, we show that the nutrient stimulus triggers a release of a tube-softening chemical agent. The propagation of the agent released at the stimulus site is flow-based, causing tube dilation downstream. We show that the organism relies on the hierarchy of the tube diameters in its network to encode and read out memories. Next, we break down the complex oscillation dynamics of $P$. polycephalum in pursuit of characteristic contraction patterns. We decompose the time series of tube contractions and identify combinations of oscillation patterns that correspond to stereotyped behaviours, such as locomotion and reaction to nutrient stimuli. Then, we turn to studying the role of calcium, the universal signalling agent. By establishing experimental protocols for measuring and quantifying calcium dynamics, we lay the groundwork for investigating calcium-related phenomena in the plasmodial network. Finally, we focus on the wound healing response in P. polycephalum. We analyze the contraction dynamics upon mechanical severing of the network and find a multi-step pattern of tube oscillations accompanying the process of wound healing.

With this work, we uncover previously unidentified functioning principles of the slime mould $P$. polycephalum, thereby contributing to the understanding of the apparent intelligent behaviour of the organism. 


\section{Contents}

Abstract

OUTLINE FOR THE READER OF THIS THESIS

LIST OF FIGURES

Author's Contributions

ACKNowledgments

Dedication

- INTRODUCTION I

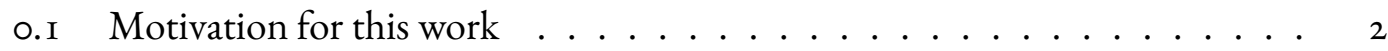

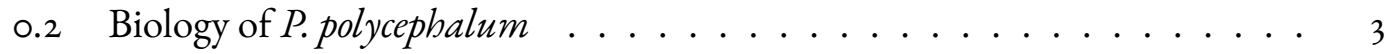

0.3 Intelligent behaviour of $P$ polycephalum .............. 9

I Morphology to ENCODE INFORMATION I I

I.I Article: Encoding memory in tube diameter hierarchy of living flow network 20

2 EMERGENCE OF BEHAVIOUR FROM NETWORK-SPANNING CONTRACTIONS $\quad \mathbf{2 7}$

2. I Article: Emergence of behavior in a self-organized living matter network . . 36

3 UNRAVELLING CALCIUM DYNAMICS IN THE PLASMOdIAL NETWORK 43

3.I Calcium as a ubiquitous signalling molecule ........... . . . . 45

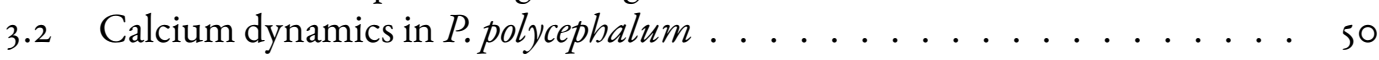

3.3 Experimental setup . . . . . . . . . . . . . 56

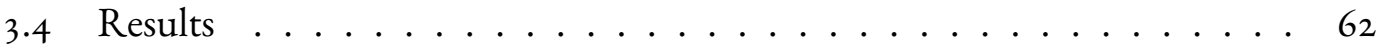

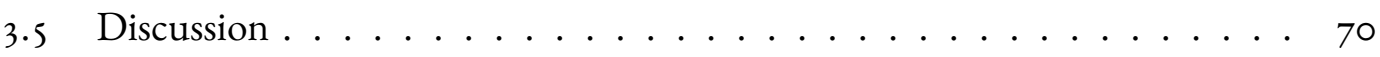


4 Network-WIDE PATTERNS OF WOUND HEALING

4. I Article: Spatial mapping reveals multi-step pattern of wound healing in Physarum

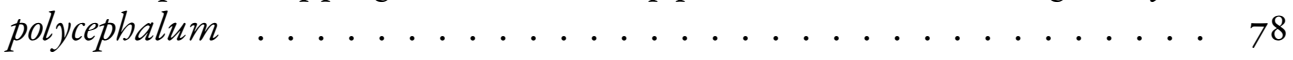

5 Conclusion And OUthoOK $\quad 85$

Appendix A

A. I Supplementary information: Encoding memory in tube diameter hierarchy of living flow network . . . . . . . . . . . . . . . . . . 92

$\begin{array}{lr}\text { Appendix B } & \text { IOO }\end{array}$

B.I Supplementary information: Emergence of behavior in a self-organized living matter network . . . . . . . . . . . . . . . . . . Ioo

ApPENDIX C 105

C.I Supplementary information: Spatial mapping reveals multi-step pattern of wound healing in Physarum polycephalum . . . . . . . . . . . . . Ios

REFERENCES

I 5 I 


\section{Outline for the reader of this thesis}

The work presented here focuses on the functioning principles of the slime mould Physarum polycephalum. With the exception of Chapter 3 which presents the development of experimental methods, the text preceding the article in each chapter contains both an introduction to the particular topic presented in the article and a discussion of the obtained results.

\section{Chapter o: InTRODUCTION}

The essential background needed to follow the discussions in individual chapters is provided. Following the motivation behind the work, a short summary of the biological properties and an overview of the intelligent behaviours of $P$. polycephalum is given.

CHAPTER I: ENCODING MEMORY IN NETWORK HIERARCHY with Karen Alim

The ability of $P$. polycephalum to encode and read out the information about the environment is presented. The idea that morphology encodes memory is introduced and the process by which this happens in the organism is studied experimentally and theoretically.

ChAPTER 2: EMERGENCE OF BEHAVIOUR FROM CONTRACTIONS with Philipp Fleig, Michael Wilczek and Karen Alim

The generation of behaviour from network-spanning contractions is studied. The rich oscillatory dynamics of the $P$. polycephalum network is broken down into distinct contraction modes to extract the basis of stereotypical behaviour.

Chapter 3: UnRaveling Calcium Dynamics

with Karen Alim

By establishing methods for calcium imaging and quantification, the experimental groundwork for studying calcium dynamics in the network of $P$. polycephalum is laid out.

Chapter 4: Patterns of Wound healing

with Felix Bäuerle and Karen Alim

In this chapter, wound healing response of the organism is studied by following the organism's response after complete severing of tubes in the plasmodial network.

Chapter 5: Conclusion and Outlook

A broad discussion of the presented work as a whole follows the short summary. 


\section{Listing of figures}

I The essential life cycle of $P$. polycephalum. The amoeba transforms into a plasmodium (I) which differentiates into sporangia (2) forming spores (3) which give rise to amoebae (4). Illustration from 'Developmental Biology of Physarum by Helmut W Sauer. Reproduced with permission of The Licensor through PLSclear. . . . . . . . . . . . . . 5 5

2 The cross-sectional contractions of a P. polycephalum's tube, schematic view. The cross section of a relaxed (a) and contracted (b) tube, depicting the invaginations of the plasmodial membrane (I), gel-like ectoplasm (2) and fluid endoplasm (3). The bundles of microfilaments (4) anchored to the plasmodial membrane shorten, thus narrowing the tube channel and displacing the endoplasm. Illustration from 'Developmental Biology of Physarum by Helmut W Sauer. Reproduced with permission of The Licensor through PLSclear. . . . . . . . . . . . . . . . . .

3 (A) Freely foraging plasmodial network exhibits characteristic shape with a dense, flared migration front and a sparse network in the back. (B) Structure of the migration front of $P$. polycephalum in a different specimen. The small channels at the tip of the migration front (top of the image) join into larger tubes towards the bulk of the network. Image in (B) with permission of Natalie Andrew. . . . . . . . . . . . . . . . . .

3.I Microinjection in a tube of P.polycephalum: two microcapillary positioning approaches. The purple microcapillary on the upper side of the tube represents the acute angle approach and the blue microcapillary on the lower side of the tube represents the perpendicular approach. The movement of the microcapillary into the plasmodial tube is depicted by the dotted line extending from the capillary tip into the tub, and its end denotes the position for microinjection. The direction of protoplasmic flows is marked by dashed arrows. . . . . . . . . . . . . . . . 
3.2 Bleaching rates of the calcium-specific dye differ in vivo and in vitro; reference dye bleaches at a lower rate than calcium-specific dye. In vitro: $10 \mu \mathrm{L}$ of $0.1 \mathrm{~mm}$ aqueous solution of Oregon Green dye alone (blue) and with $1 \mu \mathrm{L}$ of aqueous $1 \mu \mathrm{M} \mathrm{CaCl}_{2}$ solution (red). In plasmodium: total intensity of the plasmodium in calcium channel with Oregon Green dye (yellow) and reference dye Texas Red (purple). . . . . . . . . . . . . . . 6I

3.3 Distribution of reference dye Texas Red (A) and calcium-specific dye Calcium Green (B) in a growing plasmodial migration front. . . . . . . . . . . 62

3.4 The effect of $0.2 \mathrm{~mm}$ EDTA solution on the plasmodial tube in bright-field channel (first column), fluorescent reference dye channel (middle column) and fluorescent calcium dye channel (last column). The application of the chemical causes a cessation of flows and a brief elevation of calcium-channel signal before its attenuation as calcium is likely released from storage and subsequently trapped by the chelator. . . . . . . . . . . . . . . 64

3.5 Mechanical stimulus causes an increase in calcium levels. (A) A quick mechanical stimulus was applied to the tube wall, causing an increase in calcium signal immediately visible at the site of stimulation (marked by white arrow) before fading away. (B) A kymograph was recorded along the tubes traced in white. (C) Kymograph along the traced tube in calcium channel (left) and reference dye channel (right). An increase in signal is clearly visible in the calcium channel while absent in the reference channel (marked by dashed red line). . . . . . . . . . . . . . . . .

3.6 Mechanical stimulus applied to a growing migrating front causes an elevation in calcium levels and local stalling of growth. bright-field (first column), fluorescent reference dye channel (middle column) and fluorescent calcium dye channel (last column). A mechanical stimulus (marked by white arrow) causes a signal increase in the calcium channel and a stalling of fan growth at the site of elevated signal (denoted by yellow dashed line). Unaffected parts of the migrating front continue to grow at pre-stimulus rate. . . . . . . . 66

3.7 Calcium deposited by a pruning tube. (A) Position of the pruning tube in plasmodium marked by white arrow. (B) Time lapse of the pruning: functional tube at $0: \mathrm{m}:$ nmin and cessation of flow through the tube due to pruning at $30: \mathrm{m}: \mathrm{nmin}$. Bleaching causes a decrease in the signal intensity at $30: \mathrm{m}$ : nmin in the network bulk. (C) Kymograph along the length of the pruning tube. Calcium channel normalized by the reference dye channel. Dashed red line denotes the elevated calcium signal corresponding to the pruned segment. . . . . . . . . . . . . . . . 
3.8 Oscillations of calcium are in phase with the contractions in the pruning tube. (A) Tracking of the time evolution of total signal in calcium-specific and reference channel within the region of interest denoted by dashed white rectangle. (B) Signal fluctuations over time for both channels. The signal is detrended to exclude the effect of bleaching. The fluctuations in both channels are comparable in magnitude and in phase. . . . . . . . . . . 68

3.9 Relationship between calcium oscillations and contractions is consistent across different specimen and tubes, here a plasmodium with a mechanically stimulated tube shown previously. (A) Imaged section of a large plasmodium. Dashed orange rectangle denotes a tube section downstream of the mechanical stimulus, dashed blue rectangle denotes a tube unaffected by flow in the stimulated tube. (B) Fluctuation of the detrended signal in both channels in the tube segment marked in blue, stimulus time marked by dashed line. (C) Fluctuation of the detrended signal in both channels in the orange-marked tube segment, stimulus time marked by dashed line. . . . . . . . . . . . 69 


\section{Author's contributions}

This thesis contains three scientific articles, all of them included here without modifications.

The article in Chapter I is a manuscript submitted to a peer-reviewed journal, entitled Encoding memory in tube diameter bierarchy of living flow network and authored by Mirna Kramar and Karen Alim. MK and KA designed the study. MK performed experiments and numerical simulations. MK and KA wrote the manuscript. KA supervised the project.

The article in Chapter 2 is a manuscript prepared for submission to a peer-reviewed journal, entitled Emergence of behavior in a self-organized living matter network and authored by Philipp Fleig, Mirna Kramar, Michael Wilczek and Karen Alim. MK designed and performed the experiments, provided the locomotion front growth area data depicted in Fig.5-B(ii) and gave input to the final version of the manuscript.

The article in Chapter 4 entitled Spatial mapping reveals multi-step pattern of wound healing in Physarum polycephalum is published in the Journal of Physics D: Applied Physics, authored by Felix Bäuerle*, Mirna Kramar* and Karen Alim. MK developed experimental protocols and conducted the experiments. FB, MK and KA wrote the manuscript. 


\section{Acknowledgments}

Mr. Spock IS KNOWn to SAY 'Emotions ARE ALIEN to ME. I AM A SCIENTisT.'

Even though we aim to make our scientific conduct objective and impartial, the path to the Ph.D. is certainly laced with emotions. The emotion I want to express here is sincere gratitude to people who guided and supported me during this time.

First and foremost, I wish to express my deepest appreciation to my supervisor, Prof. Dr. Karen Alim for the unwavering support and guidance during the most transformative years of my life so far. A person of endless energy who does not flinch when the road gets tough, Karen taught me how to deal with challenges that research invariably imposes, and use motivation and fascination as a guiding light.

I would like to extend my sincere gratitude to my Thesis Advisory Committee: Dr. Florian Rehfeldt, Prof. Dr. Helmut Grubmüller, and Prof. Dr. Fred Wolf. I truly appreciate their support, constructive criticism and valuable advice that made me reflect and helped advance my research.

My work would not have been possible without people who provided a wonderful environment for doctoral students. I wish to thank to Antje Erdmann and Frauke Bergmann from the PBCS Office and Barbara Kutz from the MPI-DS for their kindness, patience and support. Furthermore, I would like to thank the IMPRS for Physics of Biological and Complex systems for the funding.

I would like to extend my thanks to the people whose presence in my scientific development was instrumental. Many thanks to Dr.Christian Westendorf, Dr.Natalie Andrew, Dr.Michael Wilczek, Dr.Holger Nobach, Dr.Gabriel Amselem, Dr.Mark Fricker, and Dr.Laura Turco for their knowledge, insight and advice they generously shared with me.

I had the great pleasure of working in the Biological Physics and Morphogenesis group. I deeply enjoyed the abundance or joyful, lighthearted moments and the engaging discussions. Special thanks to the Bachelor and Master students who trusted me with co-guiding them through their projects, and from whom I learned a lot. 
Those who know of my love for teaching will now understand its origin. I would like to express my love and gratitude to the following group of teachers- my family.

Želim izraziti najdublju zahvalnost roditeljima Mariju Kramaru i Matildi Kramar, sestri Margareti Kramar i baki Štefaniji Kramar na ljubavi prema znanju i poučavanju koju su mi usadili, kao i na podršci, razumijevanju i bodrenju tijekom ove avanture zvane doktorat.

Meine tiefe Dankbarkeit gilt Anne Högele-Fleig und Helmar Fleig, zwei weiteren Lehrern und meiner anderen Familie, für die Unterstützung und Ermutigung.

This section would be incomplete without two important teachers whose appreciation of science and passion for teaching made me love being a student. I want to thank Prof. Dr. Matko Milin and prof. Renata Vlaisavljević for being an inspiration.

Even though it feels fleeting, a $\mathrm{PhD}$ is a long process in which many new friendships are born, and old friendships are sustained through confelicity and commiseration. The following list is in alphabetical order because it is impossible to rank the importance of friends. Achintya Prahlad, Adrián Palacios Muñoz, Agnese Codutti, Alejandra Laguillo Diego, Aleksandra (Ola) Gebicka, Amir Bitran, Björn Kscheschinski, Brian Knep, Estefania Vidal Henriquez, Felix Bäuerle, Felix Meigel, Freja Nordsiek, George Datseris, Harald Helfgott, Hecke Degering, Jason Khadka, Jean-Daniel Julien, Jérémy Vachier, João Diniz Ramos, Komal Bhattacharyya, Laure Mancini, Maja Heuchel, Marko Popović, Matías Guiñazú, Natalie Andrew, Nicolas Lenner, Nico Schramma, Rafael Gutierrez, Sa Wu, Setareh Sharifi Panah, Steffen Werner, Tin Miladinović, and Yi Liu, thank you for all the conversations, support, good puns, bad puns, laugh attacks, and the time spent together. I cherish those memories and will carry them with me wherever I go.

And finally, I would like to thank Philipp Fleig to for walking with me every step of the way. 
Treba sabrati rojeve zvijezda

i naci sveto jedinstvo.

One should gather the swarms of stars

and find the sacred unity.

- Tin Ujević, Kozmogonije (1955)

TO FUTURE SCIENTISTS AND TEACHERS 


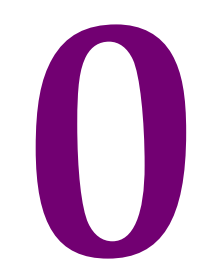

\section{Introduction}

Finding oURSELVES on the EARTH AS IT WAS A BILLION YeARS AGo WOULD LIKELy BE A SHOCKING EXPERIENCE. The predecessor of the continents, a giant rocky platform floating amid a gray ocean would look intimidating and inhospitable to life without the plethora of plants and animals inhabiting today's Earth. However, the rugged, seemingly uninviting 
environment was far from barren. Unicellular organisms already thrived in the stormy seas, the first lichens covered the craggy hills in muted colors, and at night, the ocean glistening with swarms of bioluminescent organisms was the only source of light under the heavy blanket of clouds in an atmosphere rich in carbon dioxide.

As the rapidly multiplying living beings kept on evolving under the pressure of the environment and mutual competition, multicellular life emerged and the phylogenetic tree as we know it today was developing rapidly. In the early stages, before the emergence of plants and animals, a curious organism surfaced and branched off into a separate lane. A billion years later, the surface of the planet looks completely different, but the same organism can still be found on shady forest floors. Retaining its simple build, this organism has been able to cope with the increasingly complex environment, and even demonstrate intelligent behaviour. The question that naturally comes to mind is: what is the key to its success?

\section{O.I Motivation FOR THIS WORK}

A researcher studying the slime mould Pbysarum polycephalum is in an extraordinary position. The rich behavioural repertoire of the organism, as well as its aptness for growth in the laboratory make it an ideal model organism. The slime mould has captured scientists' attention since the early days of its discovery. Looking like a fungus but capable of locomotion, it confused the botanists who attempted to classify it. As a giant syncytium, this early eukaryote exists on the verge between unicellular and multicellular life. While the ability to yield many nuclei from its cytoplasm first made it popular in cell biology and genetics, it was not until the early $2 \mathrm{I}^{\text {st }}$ century that the properties the organism is now famous for surfaced. The slime mould is capable of solving a range of complex problems known from mathematics, which 
drew researchers into studying its strategies and ultimately made the organism a template for bio-inspired design in computer science.

The network-like body of $P$. polycephalum lies at the heart of its renown problem-solving abilities. The morphology of the network is highly dynamic, allowing the organism to quickly react to stimuli and adapt to its environment. Numerous studies have tackled the mystery of $P$. polycephalum's apparent intelligent behaviour, and several phenomena have already been reproduced in theoretical models. Nevertheless, there are many open questions concerning the initiation of the observed behaviours, the properties of the signalling machinery, as well as the interplay between mechanical properties of the plasmodium and physical forces reigning inside of the giant cell.

In this thesis, we identify and address phenomena displayed by the slime mould $P$. polycephalum with the aim of uncovering the principles behind the functioning of the living network.

\subsection{Biology of P. POLYCEPHALUM}

In the recent years $P$. polycephalum has gained fame for its problem-solving abilities and made its way into being a model organism for research in biophysics and computing. However, $P$. polycephalum has already been a model organism in cell and developmental biology for over five decades ${ }^{116,141}$. Its unique properties, combined with experimental accessibility, have allowed for extensive studies of a plethora of processes in the cell, such as DNA replication, gene expression, chemotaxis, and cell differentiation which provided invaluable insight into the function of living organisms ${ }^{\mathrm{I} 39, \mathrm{I} 4 \mathrm{I}}$. 


\section{O.2.I Phylogeny}

The true slime mould Physarum polycephalum belongs to the class of slime moulds called Myxogastria within the Mycetozoa phylum, as part of the kingdom of protists ${ }^{40}$. Visually and functionally reminiscent of fungi, the organism misled the early researchers into classify-

ing it as such ${ }^{139}$. Subsequent research, as well as its growing popularity as a model organism resulted in research on $P$. polycephalum's genome, which not only allowed for correct classification, but provided insights into the evolution of eukaryotes.

Determining the sequence of small subunit ribosomal RNA and comparison with P.polycephalum's close relative, the cellular slime mould Dictyostelium discoideum revealed that $P$. polycephalum divergence occurred before D. discoideum appeared ${ }^{62}$. In 2008, Gloeckner et al. analysed roughly half of the total protein coding genes in P. polycephalum and found genes shared with Metazoa but nonexistent in D. discoideum ${ }^{43}$.

In 2016 , Schaap et al. conducted the biggest yet genome analysis in P. polycephalum, simultaneously comparing the genome with genomes of many different species. By finding $P$. polycephalum shares photoreceptor genes with bacteria, metabolic pathways with plants and a cell cycle control system with higher eukaryotes, their results provide an insight into early evolution of eukaryotes $^{\mathrm{I} 4 \mathrm{I}}$.

The fact that $P$. polycephalum possess genes of the likely last common ancestor of Amoebozoa and Metazoa ${ }^{43,141}$ paints a picture of the crossroads at a very early stage of evolutionary history, distinguishing P. polycephalum among model organisms. 


\subsubsection{LIFE CYCLE}

The essential life cycle of the slime mould P. polycephalum consists of three stages: plasmodium, amoeba and spore (Fig. I). In its plasmodial form, the organism exhibits its renowned form that has earned it the name slime mould. Plasmodium found in nature exists as an amorphous yellow mass capable of migration towards sources of nutrition and away from threat. A closer look into the plasmodial structure reveals the intricate network of channels making up a single giant multinucleate cell. In a plasmodium, cell division and nuclear division are not coupled, resulting in growth without creation of new plasmodia ${ }^{139}$.

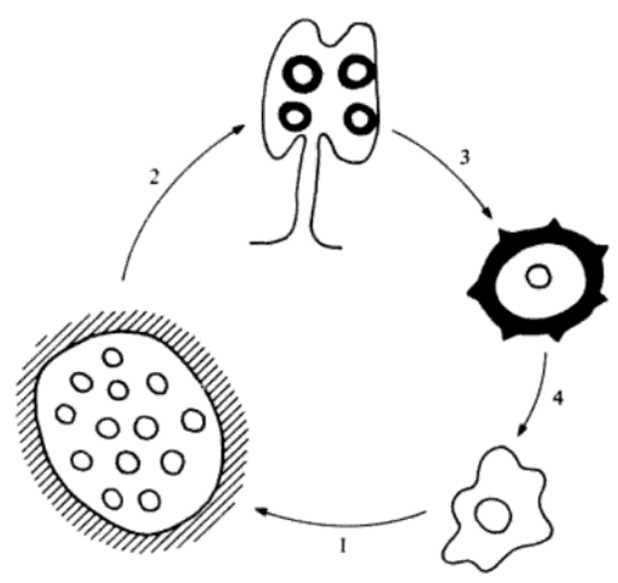

Figure 1: The essential life cycle of $P$. polycephalum. The amoeba transforms into a plasmodium (1) which differentiates into sporangia (2) forming spores (3) which give rise to amoebae (4). Illustration from 'Developmental Biology of Physarum by Helmut W Sauer. Reproduced with permission of The Licensor through PLSclear.

Unfavourable environmental conditions, such as lack of food and presence of strong light trigger a self-preserving transformation of the plasmodium. The plasmodium splits into a network of strands which form beads of plasmodial mass; the beads transform into a fruiting body- sporangium - full of spores that preserve the genetic material until the return of 
favourable conditions ${ }^{139}$.

The fruiting bodies can forcefully burst open, ejecting dry spores that spread in all directions through the air. Spores that have fallen on a suitable substrate- moist and not in direct sunlight- can break open and produce a single cell whose cell division is coupled to nuclear division, just like in regular protozoans ${ }^{139}$. This stage of the life cycle of $P$. polycephalum is known as the amoeboid stage. Unlike diploid plasmodia, amoebae are haploid and can reproduce both sexually and asexually. Fusion of haploid amoebae creates a diploid plasmodium, thus completing the life cycle ${ }^{7}$.

The full life cycle of $P$. polycephalum contains several other forms and transformations. As already mentioned, amoebae can fuse to form a zygote or proliferate, thus creating a haploid plasmodium. However, they can also differentiate and form microcysts and flagellates. Starved plasmodia can, besides into sporangia, also differentiate into macrocysts. The macrocysts embedded into a matrix of dried extracellular slime make up a sclerotium that reversibly transforms into a plasmodium upon the return of favourable conditions 3,139,140.

Even though the life cycle of $P$. polycephalum reminds of the one of Dictyostelium discoideum, the resemblance is only superficial. While $D$. discoideum never leaves its amoebal stage and instead resorts to social behaviour in forming fruiting bodies and spores, $P$. polycephalum undergoes a profound transformation in its structure and function ${ }^{139}$. Studying the mechanisms at the base of transformations between the forms in its life cycle, one can potentially come closer to understanding the transition from unicellular to multicelluar life. 


\subsubsection{STRUCTURE AND FUNCTION OF THE PLASMOdIUM}

The research conducted on P. polycephalum in this thesis has been performed solely on the organism in its plasmodial stage, thus the description of other stages in the life cycle will be omitted and the terms $P$. polycephalum, plasmodium and slime mould will be used interchangeably throughout the thesis.

Being an acellular slime mould which undergoes growth without cell division, the plasmodium of $P$. polycephalum can grow to virtually any size. While the size of the area the plasmodium covers in nature usually ranges between square millimeters and square centimeters, the organism grown in the lab can cover several square meters ${ }^{116}$.
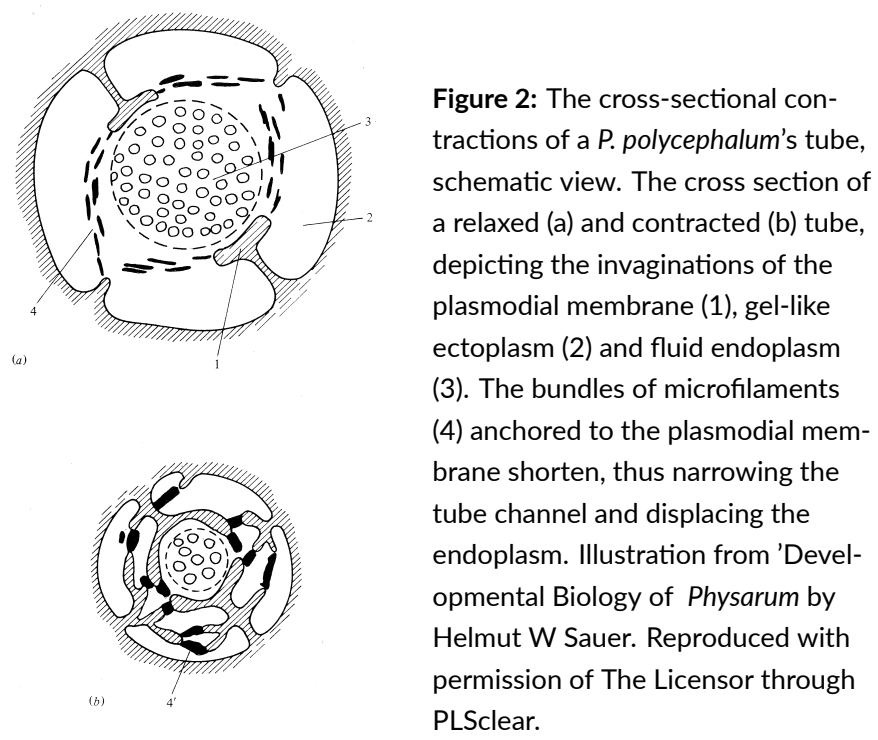

The body of the organism is made up of a characteristic network of soft, fragile tubes. The gel-like tube walls (ectoplasm) enclose a liquid protoplasm (endoplasm) that contains the nuclei and flows freely through the network ${ }^{173}$. An actin-rich actomyosin cortex is anchored to the cell membrane lining the tubes, giving the tubes the ability to contract and enabling 
locomotion of the whole organism ${ }^{\mathrm{I} 32}$.

The periodic contractions of the tubes are a distinct feature of $P$. polycephalum's. The tubes contract cross-sectionally (Fig. 2), causing a shuttle flow of the protoplasm across the network $^{21,57}$. The oscillations are organized in a peristaltic wave whose wavelength scales with the length of the organism, a phenomenon at the base of the ability of the organism to maximise transport in the network ${ }^{4}$.

Despite its simple build, the large number of different receptors $P$. polycephalum possesses indicates a remarkable awareness of the environment. The synergy of the receptors and the ability for locomotion allow the plasmodium to forage for food and recoil from threat. The organism is capable of performing chemotaxis ${ }^{73,112,168}$, phototaxis $^{49}$, thermotaxis ${ }^{98}$, and hygrotaxis ${ }^{158}$, resulting in survival abilities on par with higher organisms.

When migrating, the plasmodium has a well-defined anterior-posterior axis (Fig. 3-A) characterized by a fan-like migration or foraging front and reduced network density at the back ${ }^{106}$. The migration front appears amorphous compared to the rest of the tubular network, but a closer look reveals an intricate structure containing small interconnected channels branching off from the network body in a fractal manner and extending to the very front of the organism (Fig. 3-B). The network undergoes constant reorganization both when the organism is at stillstand and while migrating. New tubes are formed in the migration fronts by growth of the small channels, and unnecessary tubes are retracted by active pruning and relocation of tube material ${ }^{66,87}$.

The material forming the body of the plasmodium exhibits fascinating properties. The gel-like wall making up the tubes and foraging fronts is a porous ${ }^{39}$, sponge-like material, allowing the protoplasm to flow through small channels and invaginations in tube walls. Upon 
A

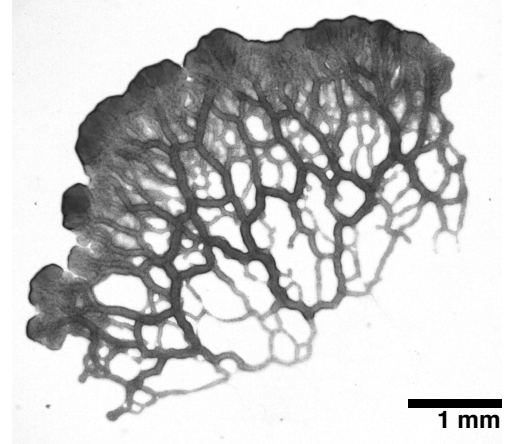

B

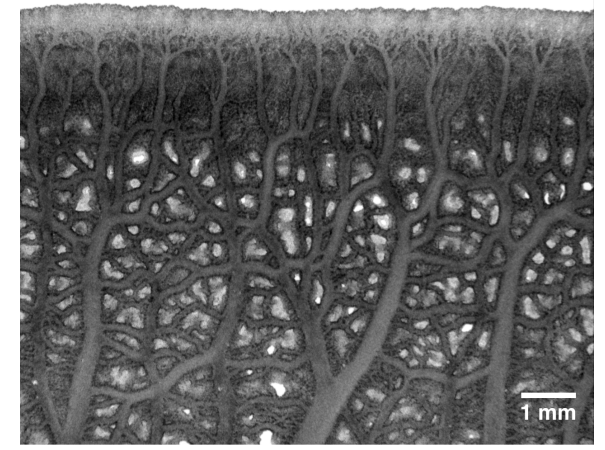

Figure 3: (A) Freely foraging plasmodial network exhibits characteristic shape with a dense, flared migration front and a sparse network in the back. (B) Structure of the migration front of $P$. polycephalum in a different specimen. The small channels at the tip of the migration front (top of the image) join into larger tubes towards the bulk of the network. Image in (B) with permission of Natalie Andrew.

external perturbation, e.g. stretching a plasmodial strand, the protoplasm can become gelatinous at the order of seconds, thereby cutting off the affected tube from the rest of the flow network $^{28,138, \mathrm{I}}$. It is likely that such features of the organism have evolved as as means of defense, minimizing damage inflicted by external factors.

The material properties and network morphology reflect the external and internal conditions alike. The network can assume staggeringly different morphologies depending on its age and starvation level, ranging from dense, lace-like networks of a well-fed plasmodium to a characteristically sparse, lightning-shaped plasmodium experiencing starvation. The transitions between the morphologies are generally reversible up to the stage of formation of fruiting bodies ${ }^{45,103,118}$.

\subsection{Intelligent BeHaviour of P. POLYCEPHALUM}

Even though its presence in cell biology and genetics research never completely ceased, $P$. polycephalum's use as a model organism declined by the end of the $20^{\text {th }}$ century. Its waning 
fame was salvaged in the early 2000's when Nakagaki et al. demonstrated the ability of the plasmodial network to find the shortest path in a maze ${ }^{105}$. This discovery has lead to numerous studies which showed that the organism's ability to solve problems transcends path finding in a maze setting. Some of the famous problems tackled successfully by the plasmodium include the travelling salesman problem ${ }^{181}$, two-armed bandit ${ }^{130}$, Steiner problems ${ }^{163}$, U-shaped trap from robotics ${ }^{\mathrm{I29}}$, towers of $\mathrm{Hanoi}^{\mathrm{I2}}$, finding optimal diet ${ }^{32,9 \mathrm{I}}$, and minimumrisk path finding ${ }^{104}$.

A famous demonstration of $P$. polycephalum's sophisticated network-building capabilities surfaced in a experiment by Tero et al. in $2010^{164}$. By arranging oat flakes used to feed the plasmodium in a spatial configuration of Tokyo railway stations, they obtained a plasmodial network closely resembling the existing network of railway lines, thus showcasing the organism's ability to create optimized networks.

The range of problems solved, combined with the elegant simplicity of the organism's function has made $P$. polycephalum a highly desirable template for algorithm design, even overshadowing ant colonies, its famous predecessor. In an extensive review article, Gao et al. provide an in-depth overview of models and computations inspired by $P$. polycephalum, ranging from cellular automata, agent-based systems and algorithms for solving differential equations such as the current reinforcement model $^{42}$. 


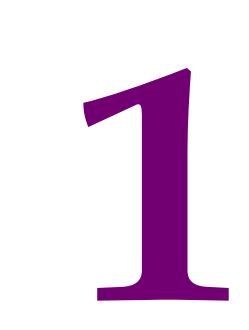

\section{Morphology to encode information}

Memory is A MULTi-FACETED PHENOMENON UNDERPINNING ALL LIFE ON EARTH.

From the tiny, blind bacteria utilizing their metabolic circuits in navigation of chemotactic gradients to the sophisticated memory systems in humans, the ability to encode, store and access stored information allows living beings to readily tackle the challenges imposed by the 
ever-changing environment.

An organism capable of internalizing the outside world increases its chances of survival and fitness ${ }^{75}$. Even though they differ in mechanisms, the phenomena arising from the pursuit of increasing fitness are surprisingly similar between starkly different species ${ }^{\mathrm{I} 45}$. The phenomena we address in this chapter are the interrelated behavioral concepts of habituation, sensitization, learning and memory. In the immense body of research on memory systems, the definitions of these key terms can differ slightly. Throughout the chapter, we will use the following definitions and later highlight the differences when making analogies between systems.

Habituation can be defined as a decrease in response to a repeated stimulus, an organismwide state following the adaptation of receptors to a stimulus ${ }^{34,172}$. The presumed evolutionary role of habituation is maintaining homeostasis by filtering out irrelevant stimuli. While the definition of habituation is conserved across phylogeny, the definition of sensitization tends to be more specific and vary between the species. However, regardless of the underlying mechanism, sensitization can be defined as increase in response to a repeated stimulus, and is presumed to aid the organism in estimating the significance of the stimulus ${ }^{34,135}$.

Habituation and sensitization can be considered two facets of the most basic form of learning, called non-associative learning ${ }^{135}$. In essence, every process resulting in maintained homeostasis or an increase of the organism fitness following a change in external conditions can be characterized as learning. If the information gained by learning is stored and can be retrieved, it can be considered as memory.

In organisms with a nervous system, memory is stored in physical changes within single neurons and in connections between multiple neurons ${ }^{127}$. Typically, behaviours arise from 
spiking activity generated by networks of neurons. The association of neurons in such activity networks is termed learning, and their preservation memory ${ }^{96}$. The enormous difference in sizes of neural networks between species is often associated with the great range of behaviours and cognitive abilities ${ }^{160}$. However, Marom et al. ${ }^{96}$ point out that the basic behavioural concepts of learning and memory are highly conserved despite the complexity of the nervous system, therefore the formation and preservation of neuronal activity networks should be based on universal principles. They argue that in order to understand how neurons produce behavioural phenomena, we must first understand the underlying universals.

The phenomena of learning and memory are not limited to organisms with a nervous system. Immobile and constantly exposed to a range of environmental stresses, plants have evolved memory encoding mechanisms not only to adapt to constant stresses, but also to tackle variable and unpredictable episodes of external perturbations ${ }^{52}$. Plants possess stressresponsive genes whose expression is different with repeated stresses ${ }^{6}$, and this transcriptional memory allows the organism to distinguish between single-occurrence and returning stresses. Another memory phenomenon in plants, but also in animals and bacteria is the epigenetic regulation, i.e. methylation of DNA as a response to stress, with the exact mechanism still a topic of ongoing debate $24,74,8 \mathrm{I}$.

A fascinating instance of memory is the immunological memory, the ability of the organism's immune system to remember the encountered pathogen in the immune cells, enabling a faster and stronger reaction at the second encounter with the pathogen ${ }^{108}$. Until very recently, it was believed that only mammals, with their adaptive antigen-specific $\mathrm{T}$ and $\mathrm{B}$ cells are capable of encoding long-term immunological memory ${ }^{88}$. However, recent research uncovered immunological memory in organisms that possess only the nonspecific innate im- 
mune system, such as lower vertebrates, invertebrates and plants ${ }^{\text {II I,I44 }}$.

In addition to the immunological memory of pathogens imprinted in the specific chemistry of the immune cells, some immune cells display the ability to encode information about their environment. Leukocytes are motile cells that successfully navigate complex chemotactic gradients by integrating conflicting signals and utilizing habituation to encode information about the current environment, resulting in an increased response to novel or remote chemoattractants $^{4 \mathrm{I}}$. Leukocytes are not alone in displaying this phenomenon; the ability to encode and store information about the environment occurs across organisms capable of chemotaxis. In bacterial chemotaxis, a well-studied instance of unicellular behaviour, Escherichia coli uses methylation of its receptors to measure ligand concentrations in a chemotactic gradient. This adaptation is dependent on the stimulus strength and sets a persistence length the organism relies on when steering its behaviour ${ }^{89, \text { I I } 4}$.

Another chemotactic organism and a close relative of the slime mould Physarum polycephalum, the social amoeba Dictyostelium discoideum is able to migrate towards the source of travelling waves of attractant molecules. The mechanism behind this phenomenon was an unsolved puzzle for a long time; previously encountered chemotactic mechanisms were insufficient to explain how cells manage to preserve their original direction. After the peak of the travelling wave of the chemoattractant has passed, an equal but opposing chemotactic gradient is formed as a consequence ${ }^{55}$. Skoge et al. propose that that the directional memory is guided by an adaptive mechanism and relies on a bistable hysteretic circuit ${ }^{147}$. The front of the wave causes a polarization through sensitization at the front and desensitization at the back of the cell. Because this internal state persists longer than the travelling wave washing over the cells, the reversal of the gradient does not cause a reorientation of the cells. 
Storing memory in hysteretic circuits is a fundamental phenomenon existing outside of the domain of life, in the realm of inanimate matter. The memory effect in magnetization of ferromagnets is a well-known example of hysteresis that enables digital memory storage ${ }^{69}$. This effect extends past magnetic materials, occurring regularly in disordered systems such as glasses $^{13}$, where the material remembers the past stresses applied to it.

The booming development of digital computation since the beginning of the last century was supported not only by the rapid technological advances, but also by the ability to harness the features of existing information processing systems in nature. The discoveries in neuroscience inspired the creation of artificial neural networks- computing systems imitating the principles that govern functions in the brain ${ }^{110}$. Just like their biological counterpart, neural networks are capable of exhibiting memory phenomena ${ }^{54}$. Even though neural networks currently hold the spotlight due to their immense potential in different applications, there are numerous computational implementations based on non-neural biological phenomena ${ }^{37}$. The abundance of inspiration that can be drawn from biological systems is well-depicted in a review article by Walker et al. ${ }^{170}$, where they elaborate on the algorithmic origins of life itself.

$P$. polycephalum is an intriguing organism frequently serving as inspiration for computational models ${ }^{42}$. The peculiarities of $P$. polycephalum, including its behaviour often labelled intelligent are described in the introduction of this thesis (Chaptero). The chemotactic properties of the slime mould do not suffice to explain the range and complexity of behaviours the organism displays, prompting a closer look into its ability to encode information.

Several memory encoding phenomena have been identified in $P$. polycephalum. The organism is capable of creating an externalized memory of its environment by sensing the previously deposited extracellular slime, which enables it to solve a classic robotics test of au- 
tonomous navigation ${ }^{129}$. Apart from the capability to create externalized memory, the slime mould also demonstrates the ability to internally encode periodic stimuli in the form of temperature or humidity changes and ultimately anticipate them ${ }^{\mathrm{I} 20}$, as well as to learn by habituation to a repellent stimulus ${ }^{17}$.

The intelligent behaviour of $P$. polycephalum hints at the possible existence of memory encoding phenomena beyond habituation.P. polycephalum-inspired algorithms are regularly based on the presumed adaptive and learning abilities of the organism ${ }^{42}$, however the the role of the central feature of the plasmodium used in these algorithms- the network of tubesremains experimentally unexplored in the context of memory encoding.

In this work, we studied the encoding of the location of a food source in the morphology of P. polycephalum network. Starting from an experimental observation of a food source imprint on the network morphology, we develop experimental and theoretical methods to study stimulus encoding. We find that the location of the food source is encoded by setting up a new hierarchy of tube diameters, that the encoding signal propagates by cytoplasmic flows. Inspired by these experimental findings, we devise a theoretical model of stimulus encoding in $P$. polycephalum network: the stimulus causes a release of a chemical agent that works to decrease the elasticity of the tube walls. The chemical agent subsequently gets advected across the network, initiating dilation of the tubes downstream of the stimulus. The theoretical model reproduces the experimentally observed tube behaviour and correctly predicts the flow-dependent response of the network. Lastly, we investigate how the encoded memories are read out to set the future migration direction of the network. We show that the organism not only strongly relies on the hierarchy of tube diameters, but also distinguishes between the tubes positioned close and far from the stimulus source. 
By showing that cytoplasmic flows are the means of encoding and reading out memories, our results reinforce the notion of cytoplasmic flows being key to network adaptation and propagation of signal ${ }^{5,164}$ in P. polycephalum. However, our results also bring out previously unidentified aspects of memory encoding in P. polycephalum. Being a foraging organism, the process of migrating and exploiting a food source are central for the function of $P$. polycephalum. By identifying the mechanism the organism uses to change its migration direction and imprint the information about the food stimulus into its body plan, we identified the missing piece of the puzzle relating signal propagation ${ }^{5}$, network adaptation ${ }^{164}$ and locomo$\operatorname{tion}^{87}$.

Furthermore, the role of tube diameter hierarchy in the encoding process elucidated in this work is in stark contrast to the existing understanding of $P$.polycephalum as an organism without an organising centre. We found that the large tubes established as a consequence of high flow rates in the plasmodial network have a purpose beyond facilitating fast advection ${ }^{95,164}$. By demonstrating the activation of the relevant tubes when reading out stored information, we showed that the organism governs its function in a highly hierarchical way, hence uncovering its internal organization.

Taking into account the already existing and newly acquired knowledge from this study, the question that naturally arises is the one about the extent of the memory encoding abilities and learning in $P$. polycephalum. Is the organism capable of more than adaptation and habituation, the non-associative forms of memory? We isolated the network plasticity of $P$. polycephalum as the essential component in the encoding process. A comparable phenomenon called synaptic facilitation can be observed in networks of neurons, where the brain temporarily enhances synaptic transmission. This complex phenomenon is presumed to play a critical 
role in information processing and formation of memories in the brain ${ }^{157}$. In their review on synaptic facilitation, Jackman et al. propose that this short-term plasticity of the network could be key to formation of long-term plasticity. In other words, synaptic facilitation creates a working memory that could be further stored as long-term memory ${ }^{60}$.

Although different in mechanism, this effect is analogous to our finding in P. polycephalum, where the network induces a short-term plasticity to create long-term memory written in the hierarchy of tube diameters and the orientation of tubes. Due to faster pruning of small tubes compared to thick transport tubes in the network ${ }^{95}$, the hierarchy established by the stimulus remains even after the stimulus is removed, indicating the existence of a long-term memory beyond adaptation - a phenomenon reminiscent of associative memory ${ }^{157}$.

In networks of neurons undergoing synaptic facilitation, the synapses act as band-pass filters and allow temporal filtering of the network ${ }^{128}$. By demonstrating the heterogeneous response of the network previously assumed to be devoid of organizing centre, we have demonstrated that the same is the case in P. polycephalum. These findings inspire exciting ideas for future research on the mechanisms $P$. polycephalum uses to process information during the encoding process.

In our experiments, the applied nutrient stimuli were not removed, allowing the network to continually reinforce its connections. The next step in studying the encoding process would be studying the network assessment of transient nutritive stimuli, as well as stimuli of different strengths. Due to the highly dynamic nature of the plasmodial network and its varying response to stimuli, as well as its reactivity to environmental changes, this is likely going to be a challenging endeavour. However, obtaining further insight into the dynamics of memory encoding process is $P$. polycephalum is a rewarding and worthwhile goal. 
With its memory-encoding abilities reminiscent of neuronal network and its simple build closer to active matter, $P$. polycephalum is an exciting organism that could serve as a template for bio-inspired design. Reinforcement learning algorithms using biological organisms as inspiration ${ }^{\mathrm{IIO}}$ can thrive on the ability of $P$. polycephalum to generate optimized transport networks, while the mechanical properties of the organism can pave the way to using $P$. polycephalum in building soft robots ${ }^{72,121,166}$. 


\title{
Encoding memory in tube diameter hierarchy of living flow network
}

\author{
Mirna Kramar ${ }^{\mathrm{a}}$ and Karen Alim ${ }^{\mathrm{a}, \mathrm{b}, 1}$ \\ ${ }^{a}$ Max Planck Institute for Dynamics and Self-Organization, Göttingen, Germany \\ ${ }^{b}$ Physik-Department, Technische Universität München, Garching, Germany
}

\begin{abstract}
The concept of memory is traditionally associated with organisms possessing a nervous system. However, even very simple organisms store information about past experiences to thrive in a complex environment - successfully exploiting nutrient sources, avoiding danger and warding off predators. How can simple organisms encode information about their environment? We here follow how the giant unicellular slime mold Physarum polycephalum responds to a nutrient source. We find that the network-like body plan of the organism itself serves to encode the location of a nutrient source. The organism entirely consists of interlaced tubes of varying diameters. Now, we observe that these tubes grow and shrink in diameter in response to a nutrient source, thereby imprinting the nutrient's location in the tube diameter hierarchy. Combining theoretical model and experimental data, we reveal how memory is encoded: a nutrient source locally releases a softening agent that gets transported by the cytoplasmic flows within the tubular network. Tubes receiving a lot of softening agent grow in diameter at the expense of other tubes shrinking. Thereby, the tubes' capacities for flow-based transport get permanently upgraded toward the nutrient location, redirecting future decisions and migration. This demonstrates that nutrient location is stored in and retrieved from the networks' tube diameter hierarchy. Our findings explain how network-forming organisms like slime molds and fungi thrive in complex environments. We here identify a flow networks' version of associate memory - very likely of relevance for the plethora of living flow networks as well as for bioinspired design.
\end{abstract}

flow networks | adaptive networks | decision making | behavior

Significance: Simple organisms manage to thrive in complex environments. Remembering information about the environment is key to take decisions. Physarum polycephalum excels as a giant unicellular eukaryote being even able to solve optimisation problems despite the lack of a nervous system. Here, we follow experimentally the organism's response to a nutrient source and find that memory about nutrient location is encoded in the morphology of the network-shaped organism. Our theoretical predictions in line with our observations unveil the mechanism behind memory encoding and demonstrate the $P$. polycephalum's ability to read out previously stored information.

\section{Introduction}

The ability to retain and access memories of past events when making decisions about future actions puts an individual into significant advantage over those lacking this ability (1). Consequently, the mechanisms of storing and recalling memories across species have evolved to be very complex (2). Even though the concept of memory has been traditionally associated with cognition (3), species devoid of nervous system also possess abilities of memory encoding. Alternative strategies of non-neuronal organisms involve metabolic or gene expression pathways. As such, organisms cope in multitude of ways, from epigenetic mechanisms and DNA inversion $(4,5)$, tunable circadian clocks (6) and cell memory during chemotaxis (7). Yet, encoding memory by gene expression pathways takes at least half an hour in simplest organisms (8) if not a full day (6), only allowing for slow decision processes. The fast decision dynamics of tens to twenty minutes of the often termed intelligent unicellular eukaryote Physarum polycephalum (9-11) suggest that so far unknown strategies to encode memory may exist. The network-shaped slime mold $P$. polycephalum is renown for its capability to mount decision that solve complex problems. P. polycephalum quickly re-organises its tubular body plan to feed itself an optimal diet among multiple nutrient sources (10, 12), to find the shortest path between nutrients in a maze (13, 14), to connect nutrient sources with an optimized transport network (9) and to solve the two-armed bandit problem (15). Pure chemotaxis toward nutrient or avoidance of undesirable territory $(16,17)$ cannot account for the complexity of problems solved. P. polycephalum's strategy to encode and read out memories about its environment remains unknown.

In the process of decision making the tubular network making up P. polycephalum's body constantly reorganizes its actomyosin-lined tubes. Actomyosin cortex drives tubes to rhythmically contract cross-sectionally (18) spatially organized in a network-spanning peristaltic wave $(19,20)$. Contractions displace the cytoplasm enclosed by the tubes thereby generating network-wide shuttle flows. Upon encountering a nutrient stimulus, tube contractions change to propagate the signal by fluid flow (21). However, the change in the contractions is transient $(21,22)$, indicating the existence of another mechanism that facilitates long-term memory encoding in the network of $P$. polycephalum.

The complex decisions of $P$. polycephalum emerge by migration of the cell or changes in the network morphology. Network morphology, in particular the hierarchy in tube diameters is controlling flow-based transport within the network $(23,24)$. Migration is itself controlled by cytoplasmic flows (25-28) and is thus also governed by network morphology. This suggest that network morphology could be key to elucidating the memory encoding abilities of the non-neural organism. 


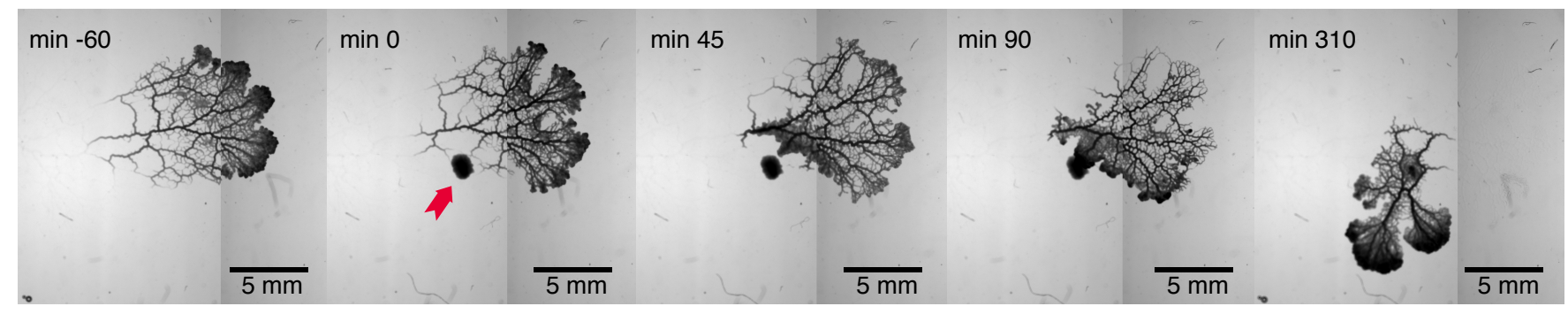

Fig. 1. Memory of a nutrient stimulus' position is encoded in network hierarchy. Bright-field images of a foraging $P$. polycephalum network subject to a localized nutrient stimulus (red arrow) applied at $0 \mathrm{~min}$. The network previously migrating to the right, reorganizes migration direction facing the nutrient within 45 min. Subsequently nutrient is exhausted, see $90 \mathrm{~min}$ until foraging is resumed, see $310 \mathrm{~min}$. Nutrient location is imprinted in the network hierarchy by thick tubes formed around the nutrient source persisting long after the nutrient is consumed.

Here, we investigate how the location of a nutrient source is encoded in the network morphology of Physarum polycephalum. By analyzing the course of a networks response to a nutrient stimulus in experiments, we find that the organism quickly imprints the stimulus by local tube dilation at the expense of other tubes shrinking, thereby setting up a new hierarchy of tube diameters. The signal to dilate tubes is propagated across the network by cytoplasmic flows resulting in a permanent change in tube diameters. Based on our observations we theoretically formulate a mechanism of encoding the location of a nutrient stimulus into the network hierarchy: the stimulus triggers the release of a chemical agent that causes the gel-like tube walls to soften, resulting in a significant dilation of tubes receiving sufficient soluble agent. The chemical agent propagates by cytoplasmic flows, initiating dilation downstream of the stimulus and thus propagating the information about the stimulus location. Numerical solution of the theoretical model correctly predicts the experimentally observed flow-dependent tube diameter response to the stimulus - generating the new network hierarchy. Finally, we show that memories encoded in network hierarchy are read out as network morphology is shaping the direction of future migration. This strategy to encode information by strengthening transport connections along the routes that stimuli propagated along reminisces of associate memories and may thus be important for living flow networks in general.

\section{Results}

Changes in tube diameter hierarchy imprint nutrient stimulus position. To study the response of $P$. polycephalum to nutrient stimuli, we collected time series of bright-field images of the organism while foraging over approximately $5 \mathrm{~h}$, during which a nutrient stimulus was applied as a single, local source in close proximity to the network (Fig. 1). Within $45 \mathrm{~min}$ after stimulus application, the organism internally re-organized to create a new migration direction facing the stimulus. Subsequently, i.e. see $90 \mathrm{~min}$ and $310 \mathrm{~min}$, the organism migrated toward the nutrient stimulus. At $310 \mathrm{~min}$ the organism almost fully consumed the nutrient source and continued to forage. Strikingly, a ring of thick tubes around the consumed nutrient source still imprinted the stimulus location in the network. This observation ignited the idea that tube diameters may encode the location of a nutrient source. However, given that $P$. polycephalum is known to make decisions even within $10 \mathrm{~min}$ to $20 \mathrm{~min}$ we next focused on the immediate response after the stimulus was applied.

To quantify network morphology and its dynamics in response to nutrient stimuli we cleared the network by trimming the specimen and analysed only networks with a stable morphology and no overt reaction to microscope light. We followed their initial reorganization during the $45 \mathrm{~min}$ after nutrient stimulus application, see Fig. 2. We subsequently analyzed the time series of bright-field images to quantify the dynamics of tube diameters. A tube designates a segment between two network vertices. With diameter we refer to the rest size of the rhythmically contracting tube, see $M a$ terials and methods and SI Appendix. The network rapidly acquires a new spatial distribution of tube diameters within about 15 min after stimulus application - a pattern that persists until the end of the experiment $(45 \mathrm{~min})$, when the organism starts to migrate towards the stimulus.

Initially, application of the stimulus causes a large-scale increase in diameter of the tubes in immediate proximity to the stimulus location and a decrease in tube volume farther away from the stimulus location (Fig. 2-A). Close inspection of the stimulus-induced relative changes in tube diameter across the network reveals spatial heterogeneity in the response: the diameters of the thick tubes directed toward the nutrient stimulus increase, while the diameters of thinner tubes in general decrease - the more the further the distance from the stimulus or the further from the thick tubes directed toward the stimulus location (Fig. 2-B). Altogether this heterogeneous response increases the hierarchy in network tube diameters. The observation that thinner tubes that are close to thick tubes do not shrink compared to those further away from thick tubes correlates with the dispersion pattern of chemicals in networks (24) and thus suggests that fluid flows based transport is at the basis of the observed network reorganization.

Fluid flow propagates stimulus into the network. To investigate if transport by flow is underlying the change in tube diameters we sort all network tubes by their Euclidean distance to the stimulus location and display their diameter dynamics over time (Fig. 3). We find that the dilation of tubes propagates in a wave-like manner from the stimulus site at the speed of $15 \mu \mathrm{m} / \mathrm{s}$, corresponding to the speed of particles advected through the network, see SI Appendix, Fig. S1. A remarkable feature of the observed process is the persistence 
A

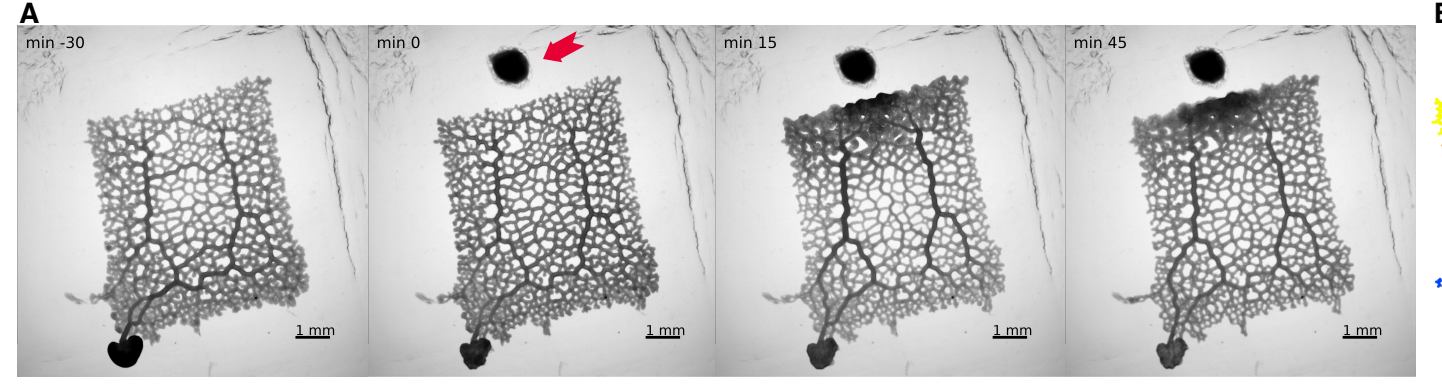

B

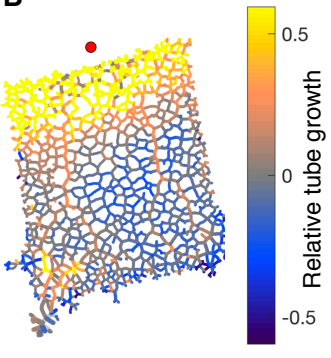

Fig. 2. Rapidly after nutrient stimulus application hierarchy of tube diameters changes establishing a new migration direction. (A) Bright-field images of a network before and after the application of a nutrient stimulus (red arrow). A new migration direction is being created at the top of the network. (B) Relative tube growth over the 45 min after stimulus (red dot) application. While overall mass is redistributed from the bottom to the top of the network, close to the stimulus site, initially larger tubes lose less mass, thus increasing network hierarchy.

of tube dilation. The new distribution of tube diameters is established within about 15 min of stimulus application and persists to the end of the experiment ( $45 \mathrm{~min}$ ).

The stability of the new tube diameters indicates a lasting change of the mechanical properties of the tube material caused by the stimulus that would allow withstanding the rapid movement of mass by shuttle flows throughout the network. The rapid and significant, up to two-fold, dilation of tubes near the stimulus site suggests that a chemical agent acts on the mechanical properties of the tubes. Agent-driven softening of tube walls would be in line with observations of migration fronts being softer than the remaining tubular network $(29,30)$.

\section{Mechanism of encoding memory in differential tube} diameter dynamics. Experiments suggest the following mechanism to encode memory of a stimulus location into network hierarchy: Upon stimulus application a soluble chemical agent gets released within the liquid cytoplasm at the stimulus location. The chemical agent softens tube wall material and thereby triggers tube dilation. This effect spreads throughout the network as the agent gets advected by cytoplasmic flows within the tubular network.

To test whether this mechanism can explain experiments we build a theoretical model with physiological parameters $(\mathrm{Ma}$ -

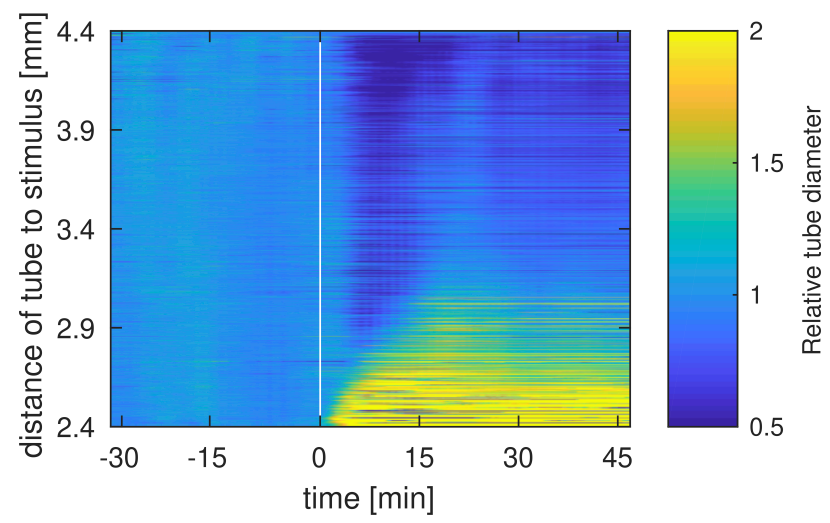

Fig. 3. Tube dilation propagates by flow transport velocity from stimulus site. Relative tube diameters throughout the network over time, individual tubes sorted by their Euclidean distance to the stimulus site. Stimulus time is denoted by white vertical line. The speed of the dilation front triggered by stimulus matches flow advection velocity of $P$. polycephalum networks. terials and methods). As the networks' response is dominantly graded with distance to stimulus site, see Fig. 3, we focus on the dynamics within a single tube of network size directed toward the stimulus site only. The cylindrical tube of radius $a(z, t)$ extends along the longitudinal axis $z \in[0, L]$. The thin shell of the active, elastic tube represents the actomyosin cortex that is enclosing the incompressible, low Reynolds number cytoplasmic fluid $\left(\operatorname{Re} \sim 10^{-3}\right)$. The cortex exerts stress $\sigma(z, t)=\sigma_{T}+\sigma_{E}$ in radial direction, where $\sigma_{T}$ is the contractile stress of the cortex activity and $\sigma_{E}$ is the elastic restoring stress of the tube wall. As the tube is long and slender, $a / L \ll 1$, the flow velocity of the cytoplasm $u(z, t)$ caused by tube contractions follows Stokes equation simplified with lubrication approximation,

$$
\bar{u}=-\frac{a^{2}}{8 \mu} \frac{\partial}{\partial z}\left(\sigma_{T}+\sigma_{E}\right) .
$$

where $\mu$ denotes cytoplasm viscosity. To describe the peristaltic contractions, the contractile stress is set to $\sigma_{T}=$ $A \cos (\omega t-k z), A$ being the amplitude of the peristaltic wave and $\omega$ its contraction frequency. The elastic stress is considered linear due to the small thickness of the tube wall compared to the radius of the tube: $\sigma_{E}=E\left(a-a_{0}\right)$, where $E$ is the elastic modulus of the tube wall and $a_{0}$ its resting radius (31). We model the softening effect of the time-averaged concentration of the chemical agent $\langle c\rangle$ on the elastic modulus as

$$
E=E_{0}-\delta E \frac{\langle c\rangle}{\left\langle c_{0}\right\rangle+\langle c\rangle},
$$

where $E_{0}$ is the elastic modulus of the unperturbed tube wall and $\left\langle c_{0}\right\rangle$ is the time-average of the agent concentration released at the site of the stimulus along the tube.

To average out concentration gradients arising solely due to shuttle flow we time-average the concentration of the chemical agent over two contraction periods, $\frac{\partial}{\partial t}\langle c\rangle=(\langle c\rangle-c) / \tau$ where $\tau$ is the characteristic timescale of the elastic modulus to respond to the stimulus. The transport of the chemical agent by fluid flow is described by Taylor dispersion $(32,33)$ which we extend by a decay term accounting for the chemical being degraded:

$$
\frac{\partial c}{\partial t}=\frac{\partial}{\partial z}\left\{-\bar{u} c+\left(\kappa+\frac{\bar{u} a^{2}}{48 \kappa}\right) \frac{\partial c}{\partial z}\right\}-k_{\operatorname{deg}} c
$$


where $\kappa$ is the molecular diffusivity and $k_{\mathrm{deg}}$ the decay rate of the chemical agent. Note that we here aim to model the very quick sensing response of the organism on the time scale of tens of minutes, which precedes the engulfment of the nutrient stimulus on the time scale of hours. Therefore, we account for a one-time release of the chemical agent due to sensing only. Finally, the total mass in the tube is conserved:

$$
\frac{\partial a^{2}}{\partial t}=-\frac{\partial}{\partial z}\left(a^{2} \bar{u}\right)
$$

giving rise to self-organised tube radii. As before we use the term diameter to refer to the rest size of the contracting tube.

\section{Memory encoding mechanism predicts flow driven tube diameter dynamics in agreement with experi-} ments. We test the memory encoding mechanism by simulating a one-time, local release of the softening agent and tracking the diameter dynamics along the closed tube as the chemical gets advected by the flow. We distinguish diameter dynamics at two segments along the tube at increasing distances from the stimulus site, see Fig. 4-A. Shortly after stimulus release close to tube end, the tube segment closest to the stimulus site dilates while the other reference segment further away decreases in diameter - due to conservation of fluid volume. Only as time progresses does the tube segment further downstream receive softening agent by flow driving it to dilate subsequently (SI Appendix, Fig. S2). Yet, the tube segment downstream does not dilate as much as the segment close to the stimulus site thereby creating a hierarchy of tube diameters in our beforehand uniform tube. The hierarchy arises as tube segments downstream receive less agent. These different softening agent concentrations are not caused by agent's decay, which is very slow $k_{\mathrm{deg}}=0.001 \mathrm{~s}^{-1}$, here, also exemplified by the long-term persistence of the dilation. Rather, the flow velocity at stimulus site is much slower than in the middle of the tube due to the eminent closed boundary at the end of the tube. Thus, most of the soluble agent stays close to it's release site while only a part gets advected causing the delayed and decreased response downstream. We next aim to make a quantitative prediction from the model that exemplifies the crucial role of flow-based transport. To this end we probe how the tube diameter response time is altered by changing flow velocities. Here, we use that contraction frequencies, which directly control flow velocities, vary naturally in P. polycephalum. We quantify as response time the time between stimulus application and minimal diameter in our tube segment further along the tube, Fig. 4-A, while decreasing contraction frequency, see Fig. 4-B. We find that the response time increases with decreasing contraction frequency, underlining the central role of flow-based transport in setting up the hierarchy in response to the stimulus.

Having in hand these two predictions on the tube diameters dynamics close and far to the stimulus and the response time we now return to our experimental data. We automatically sort tubes by their response to the stimulus (as described in SI Appendix) resulting in the two characteristic dynamics predicted by the model, see Fig. 4-C, with pure tube dilation close to the stimulus and tube shrinkage and subsequent dilation further from the stimulus. This tube behaviour is robust among all data sets (SI Appendix, Fig. S4-S8). Predicted dynamics along a single tube and experimentally observed dynamics within a network strongly resemble each other apart from two factors that arise due the simplification of a single tube in the model. First, the magnitude of dilation and shrinkage is directly tied to the total volume of the tube, see also (34), and as such smaller in the single tube compared to the voluminous network. Second, in a network, the softening agent is more and more diluted in the multitude of tubes the further it gets from the stimulus site therefore less softening agent gets to an individual tube and also gets more rapidly spread onward. This results in an on average smaller dilation and subsequent tube shrinkage. Taken these geometry-related considerations into account the resemblance of predicted dynamics and observations is striking.

Finally, we categorize our experimental data sets by contraction frequency (SI Appendix, Fig. S3) and quantify the response time across the networks (Fig. 4-D). As predicted the observed response time increases as contraction frequency decreases. And that is despite the very different morphologies and sizes of the networks. Taken together with the dynamics of tube diameters this agreement of data and prediction confirms that the advection of a softening agent by fluid flows imprints nutrient location into a specific change in hierarchy of tube diameters.

Network hierarchy is read out. Taken together our experiments and simulations show that a nutrient stimulus changes the hierarchy of tube diameters and thereby imprints its location into the network. To state that this imprint in network hierarchy poses a memory for the organism we need to test if the memory, i.e. network hierarchy, is read out. Does the network respond differently to a new nutrient stimulus when previously having encountered one in the same direction or not? Directly testing this is a futile approach as for $P$. polycephalum there are never two identical networks to compare dynamics with and without memory. However, every network comes with a given hierarchy in tube diameters, with particularly thick tubes formed in response to previous stimuli. We here use this fact to test if the memory of previous stimuli represented by thicker tubes affects the response to a new nutrient stimulus.

To visualize the spatio-temporal dynamics across a network we depict the instantaneous dilation or growth of the tubes (Fig. 5-A. This representation readily shows that there are thick tubes starting close to the nutrient source that stand out as they dilate earlier (Fig. 5-A at $7 \mathrm{~min}$ ) and more (Fig. 5-A at $10 \mathrm{~min}$ ) than their immediate neighbors and thereby enhance the hierarchy toward the nutrient source very effectively. The memory of previous events that led to these thick tubes does impact the stimulus response observed here. Given the insight gained from our model it is clear that being thick matters for the response to a nutrient stimulus as thicker tubes have higher flow velocities (24) and thus earlier receive and more effectively transport the soluble softening agent. Yet, thick alone is not sufficient as highlighted in Fig. 5-B. While 

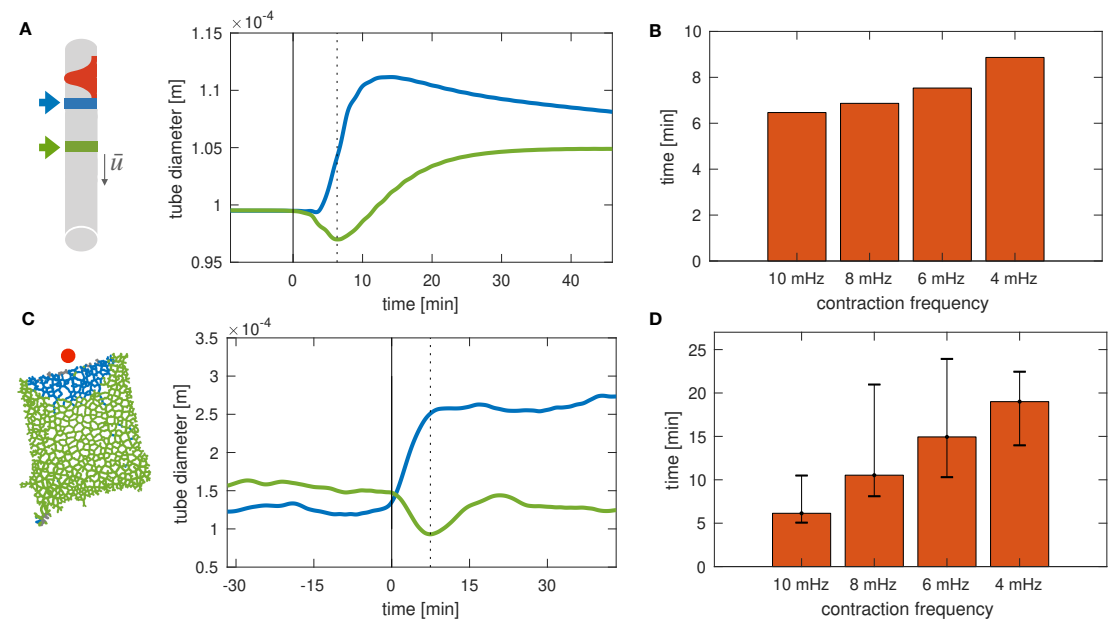

D
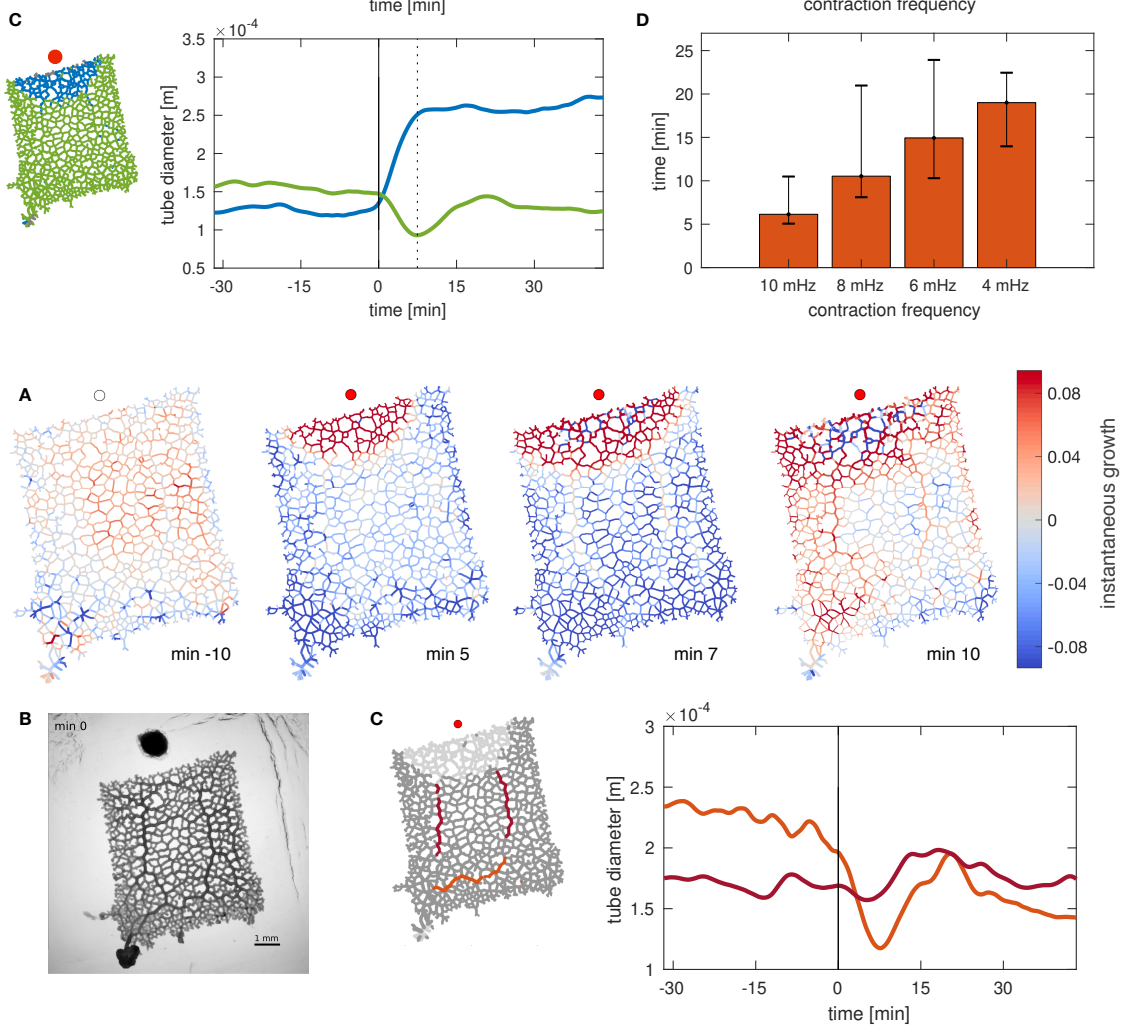

Fig. 4. Theoretical model of elastic tube dilation triggered by flow transported softening agent captures characteristic tube dynamics observed in experiment. (A) Predictions of a theoretical model of a closed peristaltic tube (sketch) on the tube diameter dynamics at the marked segments. Releasing a soluble wall-softening agent (vertical line in plot, red symbol on sketch) within an elastic tube undergoing peristalsis predicts characteristic tube dilation and relaxation. Tube dilation response time (dotted line) is shifted in time due to agent being transported by peristaltic flow. (B) A decrease in contraction frequency slowing down flows within the tube directly increases the tube segment's response time until dilation. (C) Experimental tube diameter dynamics are fully classified into the two characteristic dynamics predicted by the model (sketch). The dynamics of the mean tube diameter averaged over each ensemble of tubes follow theoretically predicted tube dynamics. (D) Quantification of the response time until dilation in experimental data sets shows increase of response time with decreasing contraction frequency, thereby showing the theoretically predicted behaviour. Error bars show the full range of tube response times in every data set.

Fig. 5. Network reads out memory encoded in network hierarchy by previous stimuli. (A) Instantaneous tube diameter dynamics reveal distinct patterns of tube dilation as dilation wave propagates into the network from stimulus site. Pre-stimulus the network is undergoing small tube diameter fluctuations. With stimulus application only tubes close by dilate while tubes elsewhere shrink. Over time tube dilation spreads into the network, notably traveling first along the tubes large in diameter and positioned close to the stimulus in flow-based travel time. (B) Brightfield image of the plasmodial network right after stimulus application. (C) Within the part of the network overall undergoing shrinkage, thick transport tubes positioned close (red) and far (orange) with respect to stimulus differ in dynamics. The average tube diameter of the tubes closer by flow-based travel time to stimulus undergo less shrinkage and recover their pre-stimulus diameter, whereas the tubes farther away shrink overall permanently. the two initially thick tubes positioned close to the nutrient stimulus grow, an even thicker tube far from the stimulus site drastically shrinks, $(\approx 50 \%)$ loss in diameter. The far away tube is far in travel time by flow from nutrient stimulus and therefore hardly receives softening agent. So memories, i.e. thicker tubes, may be overwritten as in the case of the far away tube or enhanced as in the case of tubes starting close to the stimulus site. Likely memories keep being reinforced during nutrient consumption which we do not follow here, due to the ensuing changes in network morphology adverse to quantification.

The key to why tube hierarchy represents memory and impacts organism behaviour is the flow. Flows are the mean to transport mass, to migrate toward a nutrient source. Ingeniously precisely these flows we here find to be used to enhance transport and flow toward a nutrient source by growing specifically those tubes that are quickest to be reached from the stimulus site by flow-based transport. Having thick tubes, as memories of previous stimuli, positioned close to the stimulus allow the new stimuli to spread more quickly and reorganize mass transport more efficiently.

\section{Discussion}

Our observation of a nutrient stimulus leaving an imprint on network morphology lead to the discovery of memory formation in the living flow network $P$. polycephalum. We showed that the process of stimulus encoding starts with a local softening of actomyosin-built tube walls, creating a stimulusspecific hierarchy in tube diameters. Both experimental data and numerical simulations show that softening is caused by a chemical agent transported by flows within the tubes, propagating graded tube growth across the network. While the softening agent flows through the network it reads out the existing hierarchy of tube diameters as it is encoding a new stimulus, thus using information stored by previous stimuli.

The identity of the chemical agent causing tube softening is still unknown. However, the phenomenon of actomyosin softening has been observed across living organisms, e.g. through inhibition of myosin II (35) in fibroblasts or depolymerisation of F-actin (36) in blood vessel endothelial cells. Perhaps the most likely candidate for the chemical agent is ATP, whose concentration is found to be twice as high at the migration front compared to the back of the or- 
ganism (37), the migration front formation here being a direct result of nutrient uptake.

Softening of tube walls to trigger their immediate growth is a mechanism that allows for very quick changes in tube diameter hierarchy. The timescale of less than 15 minutes observed by us now explains $P$. polycephalum's fast decisions within 10-20 minutes (9-11) that out-compete other basal organism in speed $(6,8)$. Our observations show that these quickly formed memories can be overwritten but particularly also can reinforce each other as tubes with fast flow-based travel times from stimuli keep being fueled. Finally, P. polycephalum's ability to highlight the shortest path in a maze $(13,14)$ or solving the traveling salesman problem (38) seem the logical result of memories being formed. It would be fascinating to see how different qualities or quantities in nutrient stimulus affect memory to next understand how a living flow network can adjust its architecture for an optimal diet $(10,12)$.

The novel concept that tube diameter hierarchy serves as memory not only elucidates the remarkable problem-solving capabilities of $P$. polycephalum, but also demonstrates its ability to mimic phenomena known from higher organisms, in this case synaptic facilitation (39) or synaptic plasticity (40) and reinforcement learning (41). Demonstrating the ability of the network to exhibit a phenomenon reminiscent of associative memory (42) may very well be of relevance for the plethora of living flow networks and contribute to biologically inspired design for biomimetic materials and soft robots $(43,44)$.

\section{Materials and Methods}

Culturing and imaging of $P$. polycephalum. Plasmodial networks were prepared from microplasmodia grown in a liquid culture using the medium by Daniel and Rusch (45) with hematin $(5 \mathrm{mg} / \mathrm{mL})$ instead of chicken embryo extract (46). The networks were imaged 24-36 $\mathrm{h}$ after plating on 1,5\% agar and incubated under microscope light for $1 \mathrm{~h}$ after trimming to decrease the influence of light and cutting on the tube contraction (22). The plasmodia were imaged with a Zeiss Axio Zoom V.16 microscope equipped with a Hamamatsu ORCAFlash 4.0 digital camera and a Zeiss PlanNeoFluar 1x/0.25 objective. The Zeiss Zen 2 (Blue Edition) software was used for imaging. An image was acquired every $3 \mathrm{sec}$. Stimuli were applied by placing small pellets of heat-killed HB101 bacteria on the agar using an Eppendorf ${ }^{\circledR}$ Microloader pipette tip. In two experiments presented in SI Appendix (data sets 1 and 3), $1 \mu \mathrm{L}$ of a solution containing $0.5 \mathrm{M}$ glucose and $0.1 \mathrm{M}$ leucine was placed on the agar close to the network as a nutrient stimulus.

Image analysis. First, the network skeletons, pixel intensities and tube radii dynamics were extracted using a custom written MATLAB code, see (22). Subsequently tube diameters are calculated from the time average of tube radii over 100 frames thereby averaging out radii contractions.

Numerical simulations. The equations of the theoretical model were solved numerically in a custom-written MAT-
LAB code using a theta-weighted Crank-Nicholson scheme with closed boundary conditions. Initially the tube has a uniform radius $a_{0}$. The softening agent is introduced from stimulus time onward growing exponentially in time to a final concentration of 60.0 at $4 \mathrm{~min}$. In space the stimulus is represented as a narrow Gaussian concentration profile close to the left end of the tube. The parameters used in the simulations are as follows: resting tube radius $a_{0}=50 \mu \mathrm{m}$, tube length $L=$ $0.5 \mathrm{~cm}$, contraction frequency $\omega=2 \pi / 120 \mathrm{~s}$ (47), wavelength of the travelling wave $\lambda=L$, dynamic viscosity of cytoplasm $\mu=6.4 \cdot 10^{-3} \mathrm{Ns} / \mathrm{m}^{2}$ (48), tube wall height $h=0.1 a_{0}$, contraction amplitude $A=0.1 a_{0}$, molecular diffusivity of small chemical species $\kappa=10^{-10} \mathrm{~m}^{2} / \mathrm{s}$, decay rate of the chemical agent $k_{\mathrm{deg}}=0.001 \mathrm{~s}^{-1}$. The parameters give rise to Reynolds number $R e=2 \bar{u} a_{0} / \nu \approx 0.001$. The resting elastic modulus is $E=10 \mathrm{kPa}(49,50)$, and for the feedback of chemical agent on cortex mechanics $\delta E=2.5 \mathrm{kPa}$ and $\left\langle c_{0}\right\rangle=10.0$ were chosen to result in $E=0.8 E_{0}$ at the minimum.

\section{ACKNOWLEDGEMENTS}

We thank Natalie Andrew for data set 1 in the SI Appendix. M.K. acknowledges the support of IMPRS for Physics of Biological and Complex Systems. This work was supported by the Max Planck Society.

\section{Bibliography}

1. J. S. Nairne, J. E. VanArsdall, J. N. Pandeirada, and J. R. Blunt, "Adaptive memory: Enhanced location memory after survival processing," J. Exp. Psychol. Learn. Mem. Cogn 38 no. 2, (2012) 495-501.

2. D. Shohamy and N. D. Daw, "Integrating memories to guide decisions," Curr Opin Behav Sci 5 (Oct, 2015) 85-90.

3. D. A. Redish and S. J. Y. Mizumori, "Memory and decision making," Neurobiol Learn Mem 117 (Jan, 2015) 1-3.

4. J. Casadesús and R. D'Ari, "Memory in bacteria and phage," BioEssays 24 no. 6, (2002) 512-518.

5. T. Kinoshita and M. Seki, "Epigenetic Memory for Stress Response and Adaptation in Plants," Plant Cell Physiol. 55 no. 11, (2014) 1859-1863.

6. G. K. Pattanayak, C. Phong, and M. J. Rust, "Rhythms in energy storage control the ability of the cyanobacterial circadian clock to reset," Curr Biol 24 no. 16, (2014) 1934-1938.

7. M. Skoge, H. Yue, M. Erickstad, A. Bae, H. Levine, A. Groisman, W. F. Loomis, and W. J. Rappel, "Cellular memory in eukaryotic chemotaxis," Proc. Natl. Acad. Sci. U.S.A 111 no. $40,(2014) 14448-14453$.

8. A. Amir, O. Kobiler, A. Rokney, A. B. Oppenheim, and J. Stavans, "Noise in timing and precision of gene activities in a genetic cascade," Mol. Syst. Biol 3 no. 71, (2007) 1-10.

9. A. Tero, S. Takagi, T. Saigusa, K. Ito, D. P. Bebber, M. D. Fricker, K. Yumiki, R. Kobayashi, and T. Nakagaki, "Rules for biologically inspired adaptive network design.," Science $\mathbf{3 2 7}$ no. 5964, (2010) 439-442.

10. T. Latty and M. Beekman, "Speed-accuracy trade-offs during foraging decisions in the acellular slime mould Physarum polycephalum," Proc. R. Soc. B (Feb, 2011) 539-545.

11. B. Meyer, C. Ansorge, and T. Nakagaki, "The role of noise in self-organized decision making by the true slime mold Physarum polycephalum," PLOS ONE 12 no. 3, (Mar., 2017) e0172933.

12. A. Dussutour, T. Latty, M. Beekman, and S. J. Simpson, "Amoeboid organism solves complex nutritional challenges," Proc. Natl. Acad. Sci. U.S.A 107 no. 10, (Mar, 2010) 4607 LP -4611 .

13. T. Nakagaki, H. Yamada, and Á. Tóth, "Maze-solving by an amoeboid organism," Nature 407 no. 6803 , (2000) 470.

14. V. Bonifaci, K. Mehlhorn, and G. Varma, "Physarum can compute shortest paths.," $J$. Theor. Biol. 309 (Sep, 2012) 121-133.

15. C. R. Reid, H. MacDonald, R. P. Mann, J. A. Marshall, T. Latty, and S. Garnier, "Decision-making without a brain: How an amoeboid organism solves the two-armed bandit," J. R. Soc. Interface 13 no. 119, (2016)

16. J. Smith-Ferguson, C. R. Reid, T. Latty, and M. Beekman, "Hansel, Gretel and the slime mould - how an external spatial memory aids navigation in complex environments," J. Phys. D (2017)

17. C. R. Reid, T. Latty, A. Dussutour, and M. Beekman, "Slime mold uses an externalized spatial "memory" to navigate in complex environments," Proc. Natl. Acad. Sci. U.S.A 109 no. 43, (Oct, 2012) 17490 LP - 17494.

18. N. Kamiya, "Physical and chemical basis of cytoplasmic streaming," Annu Rev Plant Physiol 32 (1981) 205-236.

19. K. Alim, G. Amselem, F. Peaudecerf, M. P. Brenner, and A. Pringle, "Random network peristalsis in Physarum polycephalum organizes fluid flows across an individual," Proc. Natl. Acad. Sci. U.S.A 110 no. 33, (2013) 13306-13311. 
20. J. D. Julien and K. Alim, "Oscillatory fluid flow drives scaling of contraction wave with system size," Proc. Natt. Acad. Sci. U.S.A 115 no. 42, (2018) 10612-10617.

21. K. Alim, N. Andrew, A. Pringle, and M. P. Brenner, "Mechanism of signal propagation in Physarum polycephalum," Proc. Natl. Acad. Sci. U.S.A 114 no. 20, (2017) 5136-5141.

22. F. K. Bäuerle, M. Kramar, and K. Alim, "Spatial mapping reveals multi-step pattern of wound healing in Physarum polycephalum," J. Phys. D 50 no. 43, (2017)

23. W. Baumgarten and M. J. B. Hauser, "Functional organization of the vascular network of Physarum polycephalum.," Phys. Biol. 10 no. 2, (2013) 026003.

24. S. Marbach, K. Alim, N. Andrew, A. Pringle, and M. P. Brenner, "Pruning to Increase Taylor Dispersion in Physarum polycephalum Networks," Phys. Rev. Lett 117 no. 17, (2016) .

25. S. Kuroda, S. Takagi, T. Nakagaki, and T. Ueda, "Allometry in Physarum plasmodium during free locomotion: size versus shape, speed and rhythm," J. Exp. Biol. 218 no. 23 , (Dec., 2015) 3729-3738.

26. S. Zhang, R. D. Guy, J. C. Lasheras, and J. C. del Álamo, "Self-organized mechano-chemical dynamics in amoeboid locomotion of Physarum fragments," J. Phys. $D$ 50 no. 20, (Apr., 2017) 204004

27. B. Rodiek, S. Takagi, T. Ueda, and M. J. B. Hauser, "Patterns of cell thickness oscillations during directional migration of Physarum polycephalum," Eur Biophys J (Apr., 2015) 1-10.

28. O. L. Lewis, S. Zhang, R. D. Guy, and J. C. del Alamo, "Coordination of contractility, adhesion and flow in migrating Physarum amoebae," J. R. Soc. Interface 12 no. 106, (Mar., 2015) 20141359

29. C. Oettmeier and H. G. Döbereiner, "A lumped parameter model of endoplasm flow in Physarum polycephalum explains migration and polarization-induced asymmetry during the onset of locomotion," PLOS ONE 14 no. 4, (2019) 1-28.

30. A. Tero, R. Kobayashi, and T. Nakagaki, "A coupled-oscillator model with a conservation law for the rhythmic amoeboid movements of plasmodial slime molds," Physica D 205 no. 1-4, (2005) 125-135.

31. K. Vajravelu, S. Sreenadh, P. Devaki, and K. V. Prasad, "Peristaltic pumping of a casson fluid in an elastic tube," J Appl Fluid Mech 9 no. 4, (2016) 1897-1905.

32. G. I. Taylor, "Dispersion of soluble matter in solvent flowing slowly through a tube," Proc. $R$. Soc. A 219 no. 1137, (1953) 186-203.

33. R. Aris and G. I. Taylor, "On the dispersion of a solute in a fluid flowing through a tube," Proc. R. Soc. A 235 no. 1200, (1956) 67-77.

34. J. Sedzinski, M. Biro, A. Oswald, J.-Y. Tinevez, G. Salbreux, and E. Paluch, "Pola actomyosin contractility destabilizes the position of the cytokinetic furrow," Nature 476 (Aug, 2011) 462

35. J. C. Martens and M. Radmacher, "Softening of the actin cytoskeleton by inhibition of myosin II," Pflugers Arch. 456 no. 1, (2008) 95-100

36. J. Fels, P. Jeggle, K. Kusche-Vihrog, and H. Oberleithner, "Cortical actin nanodynamics determines nitric oxide release in vascular endothelium," PLOS ONE 7 no. 7, (2012) .

37. T. Ueda, K. Matsumoto, T. Akitaya, and Y. Kobatake, "Spatial and temporal organization of intracellular adenine nucleotides and cyclic nucleotides in relation to rhythmic motility in Physarum plasmodium," Exp. Cell Res. 162 no. 2, (1986) 486-494.

38. L. Zhu, M. Aono, S.-J. Kim, and M. Hara, "Amoeba-based computing for traveling salesman problem: Long-term correlations between spatially separated individual cells of Physarum polycephalum," Biosystems 112 no. 1, (2013) 1-10.

39. S. L. Jackman and W. G. Regehr, "The Mechanisms and Functions of Synaptic Facilitation," Neuron 94 no. 3, (2017) 447-464.

40. R. Lamprecht and J. LeDoux, "Structural plasticity and memory," Nat. Rev. Neurosci. 5 no. 1, (2004) 45-54.

41. E. O. Neftci and B. B. Averbeck, "Reinforcement learning in artificial and biological systems," Nat. Mach. Intell. 1 no. 3, (2019) 133-143.

42. T. Takeuchi, A. J. Duszkiewicz, and R. G. Morris, "The synaptic plasticity and memory hypothesis: Encoding, storage and persistence," Philos. Trans. R. Soc. B 369 no. 1633 , (2014) .

43. K. Nakajima, T. Li, H. Hauser, and R. Pfeifer, "Exploiting short-term memory in soft body dynamics as a computational resource," J. R. Soc. Interface 11 no. 100, (2014) .

44. S. Kim, C. Laschi, and B. Trimmer, "Soft robotics: A bioinspired evolution in robotics," Trends Biotechnol. 31 no. 5, (2013) 287-294.

45. J. W. Daniel and H. P. Rusch, "The Pure Culture of Physarum polycephalum on a Partially Defined Soluble Medium," Microbiology 25 no. 1, (1961) 47-59.

46. J. W. Daniel, J. Kelley, and H. P. Rusch, "Hematin-requiring plasmodial myxomycete.," J. Bacteriol 84 no. 5, (Nov, 1962) 1104-1110.

47. J. P. Rieu, H. Delanoë-Ayari, S. Takagi, Y. Tanaka, and T. Nakagaki, "Periodic traction in migrating large amoeba of Physarum polycephalum," J. R. Soc. Interface 12 no. 106, (2015) .

48. R. Swaminathan, C. P. Hoang, and A. S. Verkman, "Photobleaching recovery and anisotropy decay of green fluorescent protein GFP-S65T in solution and cells: cytoplasmic viscosity probed by green fluorescent protein translational and rotational diffusion.," Biophys. J 72 no. 4, (Apr, 1997) 1900-1907.

49. G. Salbreux, G. Charras, and E. Paluch, "Actin cortex mechanics and cellular morphogenesis," Trends Cell Biol 22 no. 10, (2012) 536-545.

50. S. Alonso, M. Radszuweit, H. Engel, and M. Bär, "Mechanochemical pattern formation in simple models of active viscoelastic fluids and solids," J. Phys. D. Appl. Phys. 50 no. 43, (2017). 


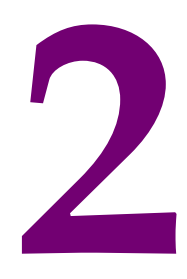

\section{Emergence of behaviour from} network-spanning contractions

THERE IS A STRIVING IN SCIENCE FOR SIMPLICITY. Ever since we started using the language of mathematics to describe natural phenomena, simple representations have been preferred 
over the complex ones and even ascribed aesthetic qualities such as beauty and elegance. As we venture further from inanimate matter into the realm of living organisms, simplification becomes a necessity due to the vast complexity underlying the behaviour of biological organisms.

As is the case with memory, the concept of behaviour is typically associated with organisms capable of cognition. Stimuli are taken in by the sensory systems and passed to the nervous system that processes them and orchestrates behaviour ${ }^{31}$. Understanding how behavior is generated from neuronal firing patterns is a coveted goal of neuroscience and great progress has been achieved since the beginnings of research on the nervous system ${ }^{27,122}$.

Even though there is an intuitive understanding, a proper definition of behaviour is necessary in order to study its generation. With the aim of mapping out behavioural sequences in the fruit fly Drosophila melanogaster, Berman et al. define behaviour as a trajectory through a high-dimensional space of postural dynamics ${ }^{\text {Is }}$. Using principal component analysis (PCA) to decompose postural dynamics, they reveal that the behaviour of the fruit fly is governed by interlinked periodic sequences of behavioural motifs, indicating an existence of low- dimensional attractors. Their findings further inspire questions about the underlying mechanisms at the neuronal level, as well as about the impact of environmental stimuli.

In a follow-up study, Berman et al. explore the multiple time scales spanned by the behaviour of the fruit fly and find a well-defined hierarchy at the base of the organisation of behavioral motifs, allowing for a prediction of future behaviour far beyond the capabilities of Markovian models ${ }^{14}$. A similar study in the worm Caenorbabditis elegans was carried out by Stephens et al. ${ }^{150}$, where they also use PCA to decompose the movement of the worm. They demonstrate a striking reduction of dimensionality by showing that approximately 
95\% of the behaviour can be described by three or four top-ranking modes, conveniently named eigenworms.

Significant advances towards the goal of understanding behaviour generation through firing patterns were made in 2016 by Nguyen et al. with their whole-brain, cell-level imaging of neuronal activity in an unconstrained C. elegans ${ }^{113}$. Combining PCA for shape dynamics characterization with cell-resolution imaging of calcium spiking patterns, they identify neurons responsible for generation of locomotory behaviour.

From the standpoint of behaviour generation, Physarum polycephalum is an interesting organism to study. Unicellular but typically existing as a spatially extended network of interconnected tubes, the organism exhibits properties of evolutionarily more advanced organisms. Its behaviour is often labelled as intelligent, as outlined in the introduction of this thesis (Cahpter o). On a more fundamental level, the organism is capable of locomotion and response to a range of external stimuli 49,87,98,158,168.

The central role of tube contractions and the resulting cytoplasmic flows in behaviour generation in P. polycephalum is by now a well-established fact and there has been tremendous progress in understanding the interplay between cytoplasmic flows and the biochemical properties of the organism ${ }^{2,46,87,92,97,117}$. However, the scope of the studies on behaviour of P. polycephalum to date is often limited by one or more of the following aspects: only a plasmodial strand or a microplasmodial droplet investigated instead of the plasmodial network ${ }^{152,178}$, just the dynamics in a fragment of the organism are observed while ignoring the spatial extent ${ }^{\mathrm{I} 42}$, or studies of the semi-steady state of a behaviour such as unbroken intervals of growth and pruning of the tubes without taking into account the onset of the behaviour ${ }^{164}$. 
The listed limitations inspire many questions about network-wide generation of behaviour in P. polycephalum. In the first study of this kind, we decompose the contraction dynamics of the tubes in the network and show that stereotyped behaviours stem from characteristic network-spanning modes of contraction. We map the contraction dynamics acquired from the bright-field time series recording of a $P$. polycephalum network on a pseudo onedimensional skeleton. We apply PCA on the resulting simplified representation of the data, obtaining a spectrum of contraction patters, i.e. modes, and their corresponding time-varying coefficients.

The continuous spectrum of eigenvalues obtained from the principal component analysis of the P. polycephalum network is in stark contrast with the results from C. elegans ${ }^{150}$, at first rendering the analysis futile. However, despite the lack of clearly defined dominant modes, the organism still exhibits coordinated contractions, end-to-end directed flow and locomotion, indicating a possible tug-of-war of contraction modes. A similarly continuous spectrum was observed in the fruit $\mathrm{fly}^{\mathrm{Is}}$, where such analysis proved useful.

A further breakdown of the results offers more insight into the coordination behaviour of P.polycephalum. Mapping the spatial structure of individual modes reveals that high-ranking modes span larger portions of the network, whereas lower-ranking modes have a fine spatial structure. We find that less than 50 modes are typically needed to reconstruct $90 \%$ of total amplitude of contractions, which is surprising given the vast number of total modes and the continuous spectrum. Furthermore, we observe that when the network uses a small number of significant contraction modes, they tend to be strongly anti-correlated, as opposed to uniform distribution of correlations values in states with high number of significant modes.

These results are interesting in several ways. The existence of intervals with high and low 
numbers of significant modes points to a possible role in behaviour. In a free, unperturbed network such as the one in our experiments, these could be the intervals of attempted directed motion of the network corresponding to low number of significant modes, and intervals of resting or internal reorganization reflected in a high number of significant modes. In order to discern between the two proposed states, we steer the behaviour of the network by applying a nutritive stimulus that reliably results in migration of the network towards the food. Interestingly, we observe a decrease in the number of characteristic contraction modes, as well as a change in their spatial structure compared to the unstimulated network. The top three significant modes, with their coefficients displaying large amplitudes, clearly dominate contraction dynamics after the application of the stimulus.

With the complexity of flow patterns that could result from the observed contraction mode dynamics, the main question about the generation of behaviour is left unanswered. To tackle this issue, we resort to a one-dimensional plasmodial strand where the flow rate is possible to obtain in addition to contraction modes. Surprisingly, even in the case of a single strand, the number of significant modes, as well as the total number of modes, is large. This result indicates that the multitude of modes does not result only from the network complexity, but in the first place from the activity of individual coupled oscillators, i.e. presumably from the contractile actomyosin rings making up the lining of the tubes ${ }^{132,155}$. However, a large flow rate through the tube can only be obtained when the number of significant modes is small. This result validates our hypothesis about locomotive and resting behaviour. It is important to note that a small number of significant modes alone does not result in high flow rates. Instead, the modes need to be correctly correlated to produce a high flow rate. In the single tube, the combination of the top-ranking significant modes produces typical ${ }^{8 \circ}$ 
plasmodial oscillation patterns, i.e. travelling waves and the standing wave.

Our results open up an array of new questions. In Physarum polycephalum, we have the unique opportunity to observe both the behaviour generation on the level of contractions as well as the behaviour of the specimen as a whole. Even though $P$. polycephalum is indisputably a much simpler system, several features that arose in this study are reminiscent of behaviour generation in neural organisms. For example, the large tubes of the network are organised in a contraction mode that is consistently among the high-ranking modes both in the unstimulated and the stimulated network. In the case of the fruit fly, where the movements of the animal are nested in sub-clusters with a clear hierarchy, Berman et al. point out that this behaviour could be a direct result of the hierarchical structure of the brain ${ }^{14}$. Even though $P$. polycephalum is often characterised as an organism without an organising centre, our results both in this study and the study of memory encoding (Chapter I) disprove that, clearly pointing out the decisive role of hierarchy in the functioning of the organism and giving way to the premise that structure dictates function. However, in order to study hierarchical relationships between the contraction modes in $P$. polycephalum and the behaviours resulting from them, further sampling is necessary.

Another intriguing feature that arose in this study is the presence of states characterized by a large number of significant modes active for a short period of time, which could also be viewed as intervals ruled by network-wide noise. Even though we did not find a proof of a behaviour specific to those states, they could still represent states of internal reorganization or change of migrating direction. Noble et al. proposed that organisms actively make use of stochasticity to generate possible solutions to the challenges the changing environment presents them ${ }^{115}$. For the case of $P$. polycephalum, Meyer et al. showed that noise helps 
the network in assessment of time-variant risk factors ${ }^{99}$. However, by introducing noise to the famous maze-solving algorithm by Tero et al. ${ }^{164}$, they probed the influence of the noise in the environment rather than the noise within the organism. The freely foraging plasmodium frequently exhibits sharp turns without obvious environmental cues ${ }^{134}$, which hints at the possibility of the organism actively harnessing its internal noise.

A question about the mechanisms generating and regulating network-wide contraction patterns arises naturally from the findings outlined above. In essence, this is a question about the nature of network-wide coupling of individual actomyosin oscillators. Takamatsu et al. compared the oscillation patterns arising from a simple system of three interconnected plasmodial fragments with results from the symmetric Hopf bifurcation, showing that the theory based only on system geometry is able to reproduce experimentally observed oscillation patterns ${ }^{155}$.

However, the mechanism behind the coupling was still a puzzle. The two most prominent candidates for network-wide coordination are the hydrodynamic coupling of cytoplasmic flows and the electrical coupling of membrane potentials. In a 20 I 7 review article, Teplov argued that hydrodynamic interactions alone should be enough to generate coordinated contractile activity along a protoplasmic strand ${ }^{162}$. In 2018 , Julien et al. modelled the generation of contractions in a $P$. polycephalum tube with a two-component model featuring an actomyosin contraction-driving chemical. Interestingly, the simulated system displays selfsustained oscillations of the cortex, which give rise to oscillatory flows. The oscillatory flows are identified as key in generating contraction waves whose wavelengths scale with system $\operatorname{size}^{63}$.

However, our results reveal a number of high-ranking network-wide oscillation patterns, 
i.e. modes, which display in-phase activity of distal areas of the plasmodium, as well as inphase contractions of large transport tubes along their whole length $(\approx 5 \mathrm{~mm})$. Furthermore, the switching between combinations of modes is very rapid. These phenomena cannot be explained by hydrodynamic coupling alone, thus hinting at the presence of another oscillator able of network-wide acting on shorter timescales than the one stemming from hydrodynamics alone.

The prime candidate for such oscillator are the synchronous oscillations of membrane potentials ${ }^{83}$. Their role in coordination of the contractions was initially ruled out after demonstrating that the cessation of the flows - but not membrane potential activity - is followed by a decoupling of tube contractions ${ }^{2}$. However, there is still the unexplained correlation between the peristaltic wave and the electric potential wave ${ }^{180}$. This correlation, the observation that the membrane potential responds to metabolic events ${ }^{84}$, and the effect of membrane potential changes on the mechanosensitive calcium channels ${ }^{44}$ in tube walls together point at a likely synergy of the hydrodynamic coupling and the membrane potential oscillations in coordinating the network-wide contractile behaviour.

Lastly, the potential role of molecular agents in the generation of behaviour should not be disregarded. By introducing a nutritive stimulus, we undoubtedly triggered a cascade of metabolic events in the organism. It is already known that several metabolites, e.g. ATP, $\mathrm{NADH}$ and $\mathrm{Ca}^{2+}$ undergo periodic oscillations with different phases and exhibit spatial patterns $^{152}$. These chemical oscillators were taken into account in poroelastic models of $P$. polycephalum droplets, yielding spiral waves and other spatio-temporal patterns in simulated plasmodial droplets ${ }^{\mathrm{I} 23,124}$. Moreover, Takagi et. al. induced a creation of rotating waves in a plasmodial droplet by applying a light pulse, a stimulus which triggers metabolic events ${ }^{153}$. 
Furthermore, Tachikawa et al. demonstrated that the nonlinearity in cytoplasm viscosity can cause symmetry breaking in a model of a plasmodial strand ${ }^{\mathrm{I}}{ }^{\mathrm{I}}$, challenging the previously assumed sole responsibility of the tube contractions for the directed flow, i.e. migration. Interestingly, they propose that a nonlinearity of cytoplasm viscosity is necessary for exhibiting behavioral modes on different timescales, i.e. tube oscillations and migration. Sato et al. showed that the viscosity of the endoplasm is oscillatory as well as a complex function of shear $^{138}$, adding to the complexity of the puzzle of behaviour generation.

Given the previous findings on hydrodynamic couplings, membrane potential oscillations, oscillations of the metabolic agents, the complexity of the endoplasm viscosity, and the newly acquired knowledge on network-wide oscillation patterns, constructing a minimal model able to reproduce the observed modes of behaviour is a challenging, but rewarding task. 


\title{
Emergence of behavior in a self-organized living matter network
}

\author{
Philipp Fleig ${ }^{\mathrm{a}, \mathrm{b}}$, Mirna Kramar ${ }^{\mathrm{a}}$, Michael Wilczek ${ }^{\mathrm{a}}$, and Karen Alim ${ }^{\mathrm{a}, \mathrm{c}}$ \\ ${ }^{a}$ Max Planck Institute for Dynamics and Self-Organization, 37077 Göttingen, Germany \\ ${ }^{b}$ Department of Physics \& Astronomy, University of Pennsylvania, Philadelphia, PA 19104, USA \\ ${ }^{\mathrm{c} P h y s i k-D e p a r t m e n t, ~ T e c h n i s c h e ~ U n i v e r s i t a ̈ t ~ M u ̈ n c h e n, ~} 85748$ Garching, Germany
}

Uncovering the origin of behavior is a challenging task. Although typically associated with a nervous system, simple life forms also show complex behavior - thus serving as a model to study how behaviors emerge. Among them the slime mold Physarum polycephalum, growing as a single giant cell, is renowned for its sophisticated foraging behavior. Here, we show how locomotion and morphological adaptation behavior emerge from self-organized patterns of rhythmic contractions of the acto-myosin lining the tubes of the network-shaped organism. We quantify the spatio-temporal contraction dynamics by decomposing experimentally recorded contraction patterns into spatial contraction modes. Surprisingly, we find a continuous spectrum of modes, as opposed to few dominant modes. Over time, activation of modes along this continuous spectrum is highly dynamic resulting in contraction patterns of varying regularity. We show that regular patterns are associated with stereotyped behavior by triggering a behavioral response with a food stimulus. Furthermore, we demonstrate that the continuous spectrum of modes and the existence of irregular contraction patterns persist in specimens with a morphology as simple as a single tube. Our data suggests that the continuous spectrum of modes allows for dynamic transitions between a plethora of specific behaviors with transitions marked by highly irregular contraction states. By mapping specific behaviors to states of active contractions, we provide the basis to understand behavior's complexity as a function of biomechanical dynamics. This perspective will likely stimulate bio-inspired design of soft robots with a similarly rich behavioral repertoire as $P$. polycephalum.

ethology | flow network | unicellular | self-organization | living matter

Significance: In living systems behavior arises from underlying seemingly impenetrable complex processes. It is therefore common to apply a top-down approach: first find stereotyped behaviors; then seek to understand their individual emergence. The nature of the living matter network $P$. polycephalum allows the reverse approach: we analyze the dynamics of the living matter and thereby explain the emerging observed behavior. We show that a surprisingly broad distribution of dynamic states empowers a rich plethora of behavior. Each single behavior is associated with a set of few dominating dynamic states, yet transitions are marked by a large number of equally important states. This bottom-up insight into the emergence of behavior opens up new perspectives in biomimetics and soft robotics.

\section{Introduction}

Survival in changing environments requires from organisms the ability to switch between diverse behaviors $(1,2)$. In higher organisms, rapid changes in neural activity enable this capacity, ranging from almost random to strongly correlated firing patterns of neurons $(3,4)$. However, many organisms without a nervous system are also able to readily transition between a multitude of behaviors, which suggests that the underlying biophysical processes display a dynamic range analogous to that of a nervous system.

Examples of specific behaviors in non-neural organisms include the run-and-tumble chemotaxis of the E.coli bacterium (5), environmentally cued aggregation of the social amoeba Dictyostelium discoideum $(6,7)$ and the cooperative growth behavior of B.subtilis bacterial colonies $(8,9)$. An organism with an exceptionally versatile behavioral repertoire is the slime mould Physarum polycephalum - a unicellular, network-shaped organism (10) of macroscopic dimensions, typically from a millimeter to tens of centimeters.

$P$. polycephalum's complex behavior is most impressively demonstrated by its ability to solve spatial optimization and decision-making problems (11-15), habituation to temporal stimuli (16), exploration versus exploitation strategy (17).Such behavior, comparable to that of some higher organisms, implemented on a macroscopic scale, requires a mechanism that allows for long-range spatial coordination.

Characteristic for P. polycephalum are the active, rhythmic, cross-sectional contractions of the actomyosin cortex lining the tube walls (18-20). The contractions drive cytoplasmic flows throughout the organism's network $(21,22)$, transporting nutrients and signalling molecules (23). Cytoplasmic flow is responsible for mass transport across the organism and thereby contractions directly control locomotion behavior (24-28).

So far only the most dominant network-spanning peristaltic contraction wave $(22,29)$ has been described experimentally. However, for small $P$. polycephalum plasmodial fragments various other short-range contraction patterns have been observed $(25,26)$ and predicted by theory of active contractions (30-34). Similarly, up to now unknown complex, large-scale spatio-temporal contraction patterns might explain some of $P$. polycephalum's behavior.

Here, we decompose experimentally recorded contractions of a large $P$. polycephalum network of stable morphology into a set of physically interpretable contraction modes using Principal Component Analysis. Surprisingly, we find a continuous spectrum of modes and high variability in the activation of modes within this spectrum. By perturbing the network with an attractive stimulus, we show that the resulting loco- 
A

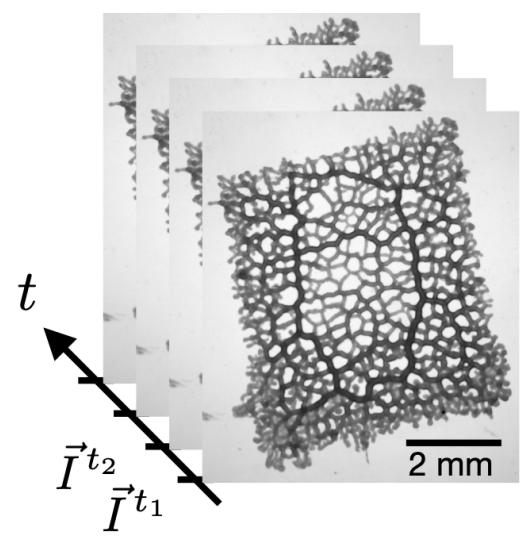

C
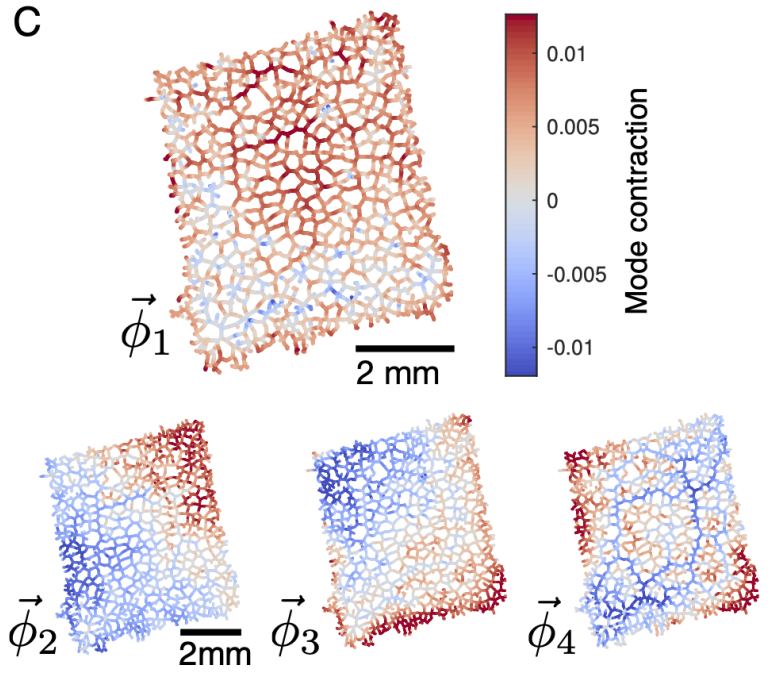

B

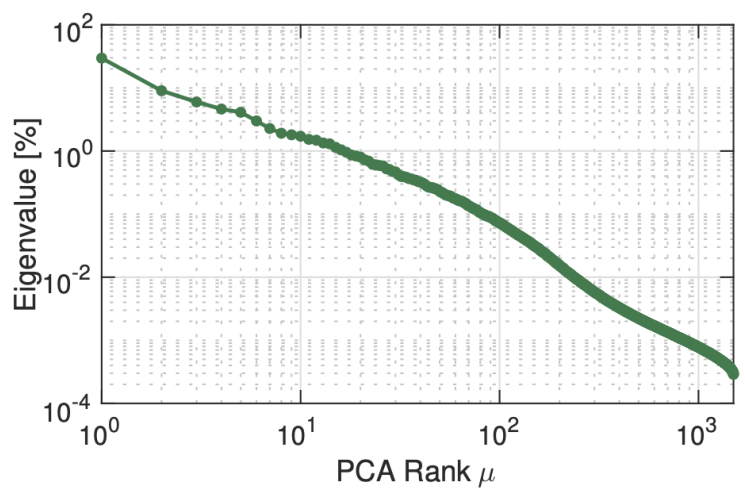

(ii)

$\mathrm{T} \sim 100$ seconds

$a_{1}$
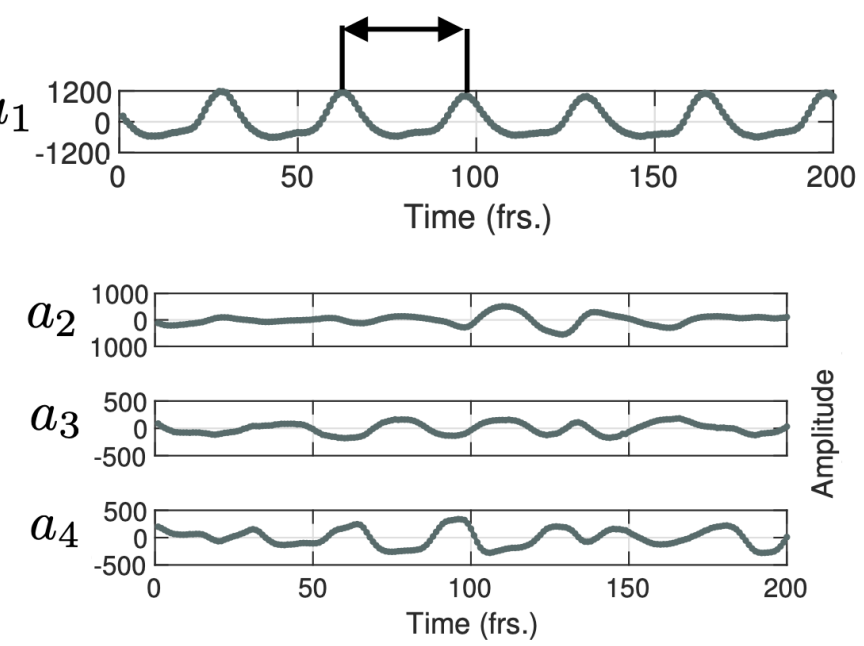

Fig. 1. Principal Component Analysis yields a continuous spectrum of contraction modes of the P. polycephalum network. (A) Exemplary stack of bright-field images of the recorded network. Pixel intensities encode the contraction state (tube dilation) at each point of the network. Principal Component Analysis is performed on a stack of post-processed bright-field frames. (B) Relative eigenvalues in percent, plotted against the mode rank $\mu$ on a log-log graph. The eigenvalue spectrum is continuous, without a natural cutoff. (C) (i) Structure of the four highest-ranking modes $\vec{\phi}_{1,2,3,4}$ with their respective coefficients shown in (ii). The red-blue color spectrum indicates the contraction state. The modes are eigenvectors of the covariance matrix. The coefficient $a_{1}$ of the first mode captures the organism's characteristic oscillation period of $\sim 100$ sec, while the coefficients $a_{2,3,4}$ show considerable variation in amplitude and frequency over time.

motion response is coupled to a selective activation of regular contraction patterns. Guided by these observations, we design an experiment on a $P$. polycephalum specimen reduced in morphological complexity to a single tube. This allows us to quantify the causal relation between locomotion behavior, cytoplasmic flow rate and varying types of contraction patterns, thus revealing the central role of dynamical variability to generate different behaviors.

\section{Results}

Continuous spectrum of contraction modes reveals large variability in organism's contraction dynamics.

To characterize the contraction dynamics of a $P$. polycephalum network, we record contractions using bright-field microscopy and decompose this data into a set of modes using Principal Component Analysis (PCA). At first, networks in bright-field images are skeletonized, with every single skeleton pixel representing the local tube intensity as a measure of the local contraction state (35). Thus, any network state at a time $t_{i}$ is represented by a list of pixels, $\vec{I}^{t_{i}}$, along the skeleton, see Fig. 1A. Performing PCA on this data results in a linear decomposition of the intensity vectors $\vec{I}_{i}$ into a basis of modes $\vec{\phi}_{i}$ (SI Text):

$$
\vec{I}^{t_{i}}=\sum_{\mu} a_{\mu}^{t_{i}} \vec{\phi}_{\mu} .
$$

The modes, $\vec{\phi}_{i}$, are orthonormal eigenvectors of the covariance matrix of the data and represent linearly uncorrelated contraction patterns of the network. $a_{\mu}^{t_{i}}$ denotes the timedependent coefficients of the modes.

We rank modes according to the size of their eigenvalue. Contrary to the small number of large eigenvalues found in a number of biological systems (36-38), here the spectrum of relative eigenvalues (SI Text) reveals a continuous distribution of eigenvalues with no natural cutoff Fig. 1B. As a result, PCA does not directly lead to a dimensionality reduction of the data. Instead, we here investigate the special characteristics of mode dynamics that result from a continuous spectrum and how these shape the organism's behavior.

The highest ranking modes shown in Fig. 1C(i) have a smooth spatial structure that varies on the scale of network size. As we will discuss below, such large scale modes are associated with the long wavelength peristalsis observed 

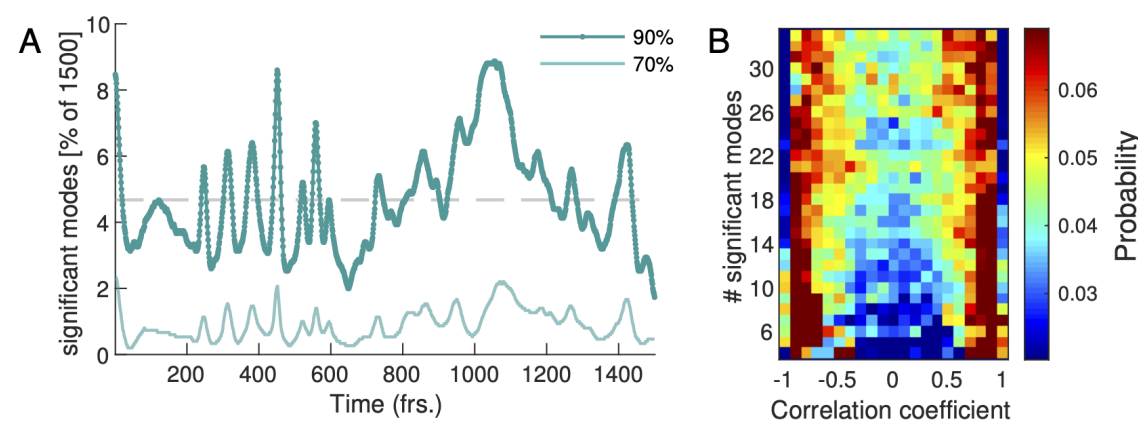

Fig. 2. Dynamics of network contraction pattern is subject to strong variability in the percentage of significant modes and correlations between them. (A) Significant modes given by the percentage of the total number of modes required for the cumulative sum of their relative amplitudes to reach $70 \%$ (light green) and $90 \%$ (dark green) of the total amplitude plotted over time. Gray dashed line indicates the $4.68 \%$ ( $\approx 70$ modes) average of significant modes. (B) Distribution of coefficient correlation values depending on the number of significant modes taken from the $70 \%$-cutoff curve in (A). Correlation values show a trend from strong (anti-)correlation for a small number of significant modes (lower) to a more uniform distribution of correlation values for a large number of significant modes (upper). in $(21,22)$. Interestingly, we also find modes highlighting specific morphological characteristics of the network. The structure of mode $\vec{\phi}_{4}$ corresponds to the thickest tubes of the network, c.f. Fig. 1A, which suggests a special role of these tubes in the functioning of the network. Finally, as we go to lower and lower mode rank, the spatial structure of the modes becomes finer and finer. Yet, despite lacking an obvious interpretation for their structures, like for mode $\vec{\phi}_{30}$, SI Appendix Fig. S1, it is not possible to ignore their contribution relative to high ranking modes.

Next, we turn to the time-dependent coefficients of modes shown in Fig. 1C(ii). In accordance with the known rhythmic contractions (39) the coefficient $a_{1}$ of the highest ranked mode $\vec{\phi}_{1}$ oscillates with a typical period of $T \sim 100 \mathrm{sec}$. Most strikingly, amplitudes of mode coefficients vary significantly over time - even on orders of magnitude.

To map out the complexity of contractions over time, we define a set of significant modes for every time point. We quantify the activity of a mode by its relative amplitude

$$
p_{\mu}^{t_{i}}=\frac{{\widetilde{a^{2}}}_{\mu}^{t_{i}}}{\sum_{\nu}{\widetilde{a^{2}}}_{\nu}^{t_{i}}},
$$

where $\widetilde{a_{\mu}^{2}}$ denotes the amplitude of the square of the coefficient. By definition the sum over the relative amplitudes of all modes is normalized to one at any given time, $\sum_{\mu} p_{\mu}^{t_{i}}=1$. We order the modes by their relative amplitude from largest to smallest and take the cumulative sum of their values until a chosen cutoff percentage is reached, see Fig. 2A. We find that the percentage of modes required to reach a specified cutoff value varies considerably over time. For a $90 \%$ amplitude cutoff we find that on average $6.06 \%$ ( $\approx 70$ modes) of the 1500 modes are significant with a large standard deviation of $36.96 \%$.

Apart from the number of significant modes, the dynamics of the network depends on the temporal correlation of modes. In Fig. 2B we show the distribution of temporal correlations between mode coefficients as function of the number of significant modes Materials and Methods. For a small number of significant modes the coefficients are strongly (anticorrelated in time, while for a large number of significant modes, temporal correlations between coefficients are more uniformly distributed over the entire interval. The first reflected in coordinated pumping behaviour/contractions, and the latter giving rise to irregular network-wide contractions. The above analysis shows that the dynamics of network contractions covers a wide range in complexity, from superposition of few large-scale modes strongly correlated in time, to superpositions of many modes of varying spatial scale and temporal correlations. This gives rise to strong variability in the regularity of the contraction dynamics over time. For now we investigated an 'idle' network not performing a specific task, so we next stimulate the network to provoke a specific behavior and scrutinize how the continuous spectrum of modes contributes to it.

\section{Stimulus response behavior is paired with activation of regular contraction patterns interspersed by many-} mode states. To probe the connection between a specific behavior and network contraction dynamics we next apply a food stimulus to the same network, see Fig. 3A. Food acts as an attractant and causes locomotion of the organism toward the stimulus in the long term. The stimulus immediately triggers the network to inflate in a concentric region around the stimulus site. Also, the thick transport tubes oriented toward stimulus location increase their volume, see Fig. 3A(iii). Altogether these morphological changes are typical for the specific behavior induced here, namely the generation of a new locomotion front.

To identify potential changes in the contraction dynamics due to stimulus application we perform PCA on a 700 frames long subset of the data subsequent to the data of the previous section. At first, we rediscover a continuous spectrum of modes displaying large variability in the number of significant modes resembling the 'idle' dynamic states, see $S I$ Appendix Fig. S2. Yet, when mapping out the spatial structure of the highest-ranked contraction modes, see Fig. 3B, we discover that the now dominant modes clearly reflect the visually determined response of inflation close to the stimulus and the activation of the thick transport tubes. In fact, for more than 500 frames after the stimulus has been applied, the rhythmic contraction dynamics of the network are dominated by the three highest ranked modes, see Fig. 3C and SI Appendix Fig. S3 for the mode coefficients. During this period, every time a single mode is the most active one for a duration of $>30$ frames, its amplitude exceeds that of any other mode by $20-30 \%$.

Taken together, we observe the imprint of the generation of a new locomotion front, as after stimulus application new 
A

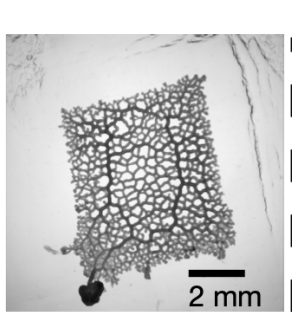

frame 1650

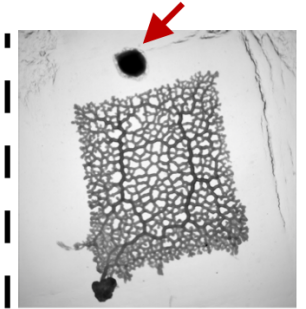

fr. 1675

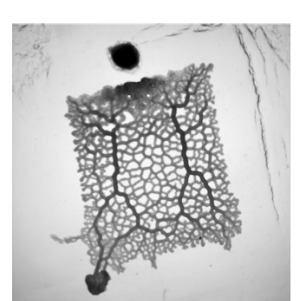

fr. 2150
B

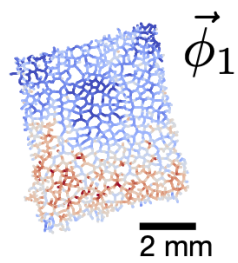

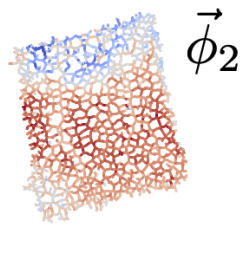

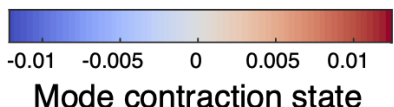

C
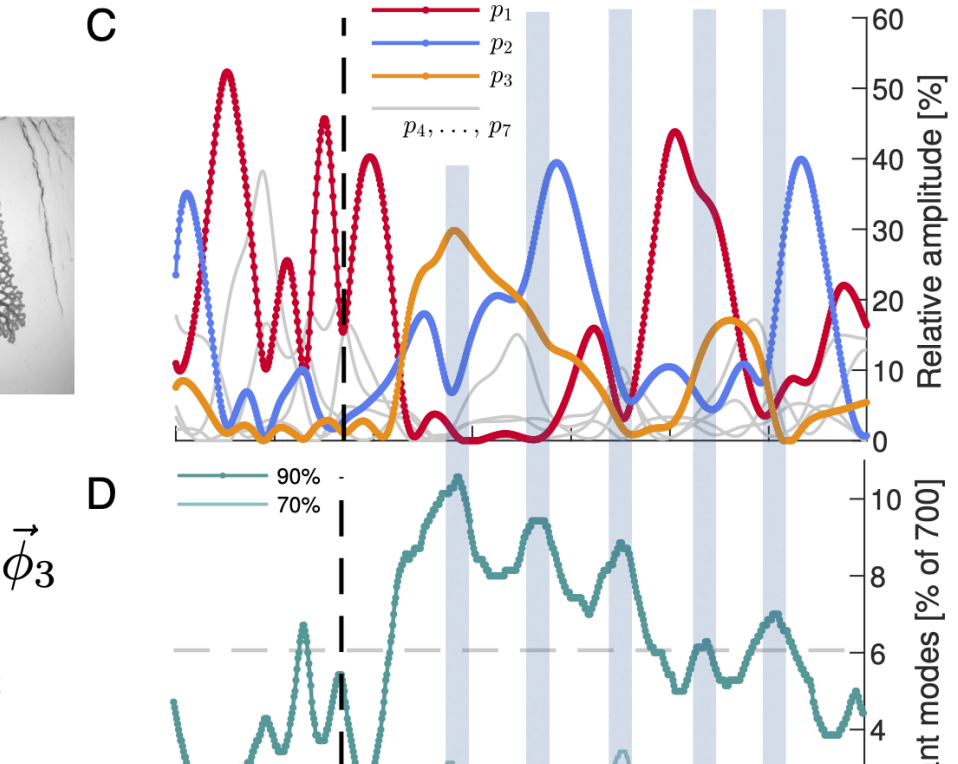

D

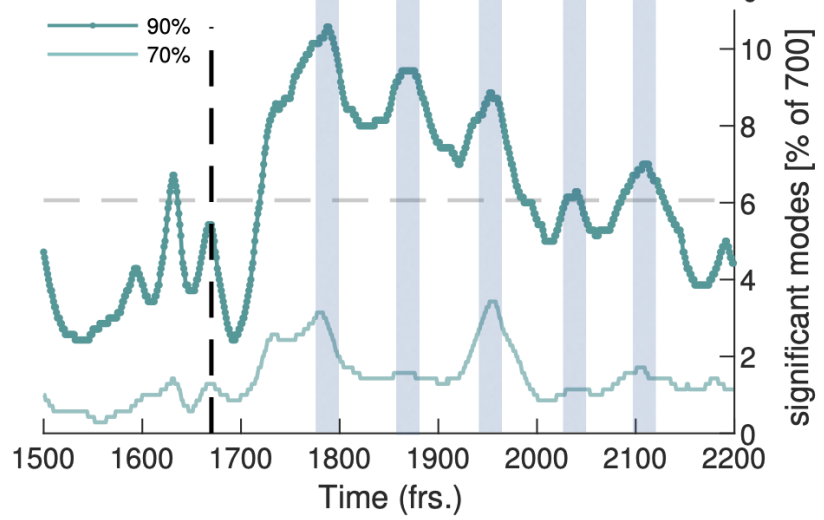

Fig. 3. Network reacts to stimulus with variations in the dynamics of the contraction pattern. (A) Bright-field frames showing the network's growth response to a food stimulus (red arrow). (B) The spatial structure of the three top-ranked modes. (C) Dynamics of the relative amplitudes of the three top-ranked modes. After the stimulus (dashed line at frame 1670) time intervals with a single contraction dominating in amplitude (red, blue, yellow) over all other modes, mode amplitudes four to seven shown in gray for reference. Interspersed we find time intervals where a larger number of modes have a similar amplitude. These times are indicated by the blue shaded boxes extending across (C) and (D). (D) Percentage of total number of modes required to reach a cumulatively summed amplitude of $70 \%$ (light green) and $90 \%$ (dark green) of the total amplitude, over time. Gray dashed line indicates the $6.06 \%(\approx 42$ modes) average of significant modes.

stimulus-specific modes like $\vec{\phi}_{2}$ and $\vec{\phi}_{3}$ emerge and dominate with very regular contraction dynamics for extended phases of time. Strikingly, these regular contractions dynamics are interspersed with many-mode states where the number of significant modes increases considerably, see Fig. 3D. The number of significant modes seems to oscillate after the stimulus and distinct maxima, of many modes, coincide with times at which the organism switches from one dominant contraction pattern to another, as indicated by the shaded regions in Fig. 3C and Fig. 3D. While our observations suggest that prolonged regular dynamics dominated by a few or even a single mode are associated with specific behavior like locomotion, the many-mode states seem to serve as transition states between them. While the network morphology is characteristic for $P$. polycephalum, reducing network complexity may help to conclude on the role of regular dynamics, manymode states and the therefrom arising continuous distribution of modes.

\section{Number of significant modes determines maximum cytoplasmic flow rate in the minimal morphological representation of the network. We next perform exactly} the same course of experiments as before but on a $P$. polycephalum specimen reduced in complexity to a single tube with a locomotion front at either end, see inset in Fig. 4. Strikingly, again we find a continuous spectrum of modes displaying a large variability in the number of significant modes, see SI Appendix Fig. S4 and S5. This observation finally un- derlines that the continuous spectrum of modes and its variability in activation is intrinsic to the organism's behavior, ruling out that the complexity of contraction modes only mirrors morphological complexity. Foremost, this minimal network allows us now to directly map the effect of variations in the contraction dynamics onto behavior. From the experimentally quantified tube contractions we calculate the maximal flow rate at any point of the tube (40) and correlate the flow rates driving locomotion at the tube ends with the number of significant modes at a time, see SI Appendix. For both

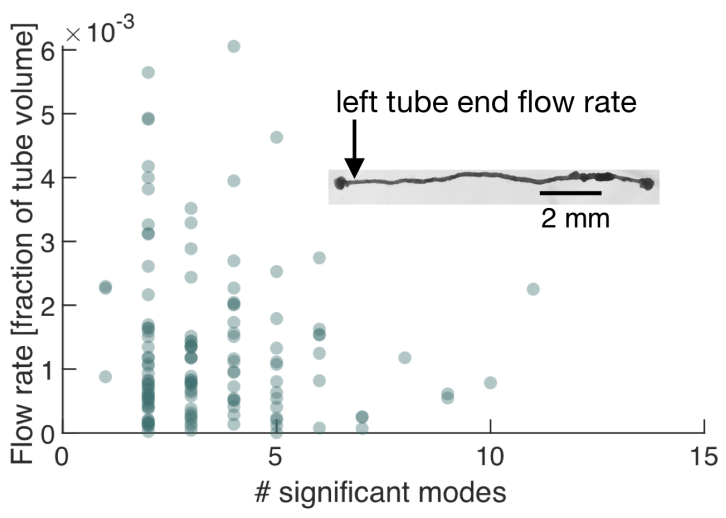

Fig. 4. Number of significant modes is indicative for the volume flow rate in a cell reduced in its network complexity to a single tube. Inset: Single tube with locomotion fronts at both ends. Main plot: Volume flow rate at the left tube end, calculated from tube contraction dynamics versus the number of significant modes at different times. High flow rates are only achieved for a small number of significant modes. 
the flow rate at the left and right end of the tube, shown in Fig. 4, and SI Appendix Fig S6., respectively, we find that large flow rates are only achieved when the number of significant modes is small. We had previously found that few significant modes are highly (anti-)correlated, whereas states with many significant modes are not, see Fig. 2. This observation now confirms our physical intuition that the irregularity of states consisting of many modes goes hand in hand with reduced pumping efficiency and thus unspecific behavior. Since a small number of significant modes not necessarily always implies a large flow rate, we next turn to analyse their exact spatial structure and instantaneous temporal correlation to determine how cytoplasmic flow rates impact behavior.

\section{Instantaneous coupling and selective activation of modes determine locomotion behavior. We now demon-} strate the impact of changes in the dynamics of a small number of modes on the organism's behavior. For this we quantify the locomotion behavior of the single tube by tracking the area of the locomotion fronts protruding from each end of the tube over time, see Fig. 5B(ii). While initially the right end is protruding faster at the expense of the left end, a food stimulus applied to the left end of the tube flips the direction of locomotion.

Turning to the modes that drive this behavior, we find that over long time intervals, and in particular after the stimulus, the two top-ranked modes dominate the tube's contraction dynamics, see Fig. 5(B)(i) and SI Appendix Fig. S4 and S7. Parametrizing the single tube with a longitudinal coordinate, the spatial shape of these dominating modes $\vec{\phi}_{1}$ and $\vec{\phi}_{2}$ approximate Fourier modes, see Fig. 5(A). To illustrate the connection between tube contraction dynamics and locomotion behavior, we pick two representative time intervals after the stimulus where either only mode $\vec{\phi}_{1}$ or modes $\vec{\phi}_{1}$ and $\vec{\phi}_{2}$ equally dominate overall, see vertical pink bars in Fig. 5(B). During the first interval when the mode $\vec{\phi}_{1}$ alone is dominating, the tube is driven by a standing wave contraction pattern - yielding only a low cytoplasmic flow rate. Correspondingly, the size of the locomotion front at either end shows no significant change in area during this interval. In contrast, during the interval when both modes $\vec{\phi}_{1}$ and $\vec{\phi}_{2}$ are equally active, the resulting superposition is a left-traveling wave producing a large cytoplasmic flow rate in that direction. The lefttraveling wave is in accordance with the growth of the left and retraction of the right locomotion front. These two examples solve the conundrum of Fig. 4, which shows that a small number of significant modes does not necessarily lead to high cytoplasmic flow rates. The direct mapping of contraction dynamics onto ensuing cytoplasmic flows confirms that a small number of significant modes is associated with specific behavior. High cytoplasmic flow rates at the tube ends drive locomotion, while lower flow rates likely lead to other behaviors such as mixing. Furthermore many-mode states seem necessary for transitions in a multi-behavioral space.
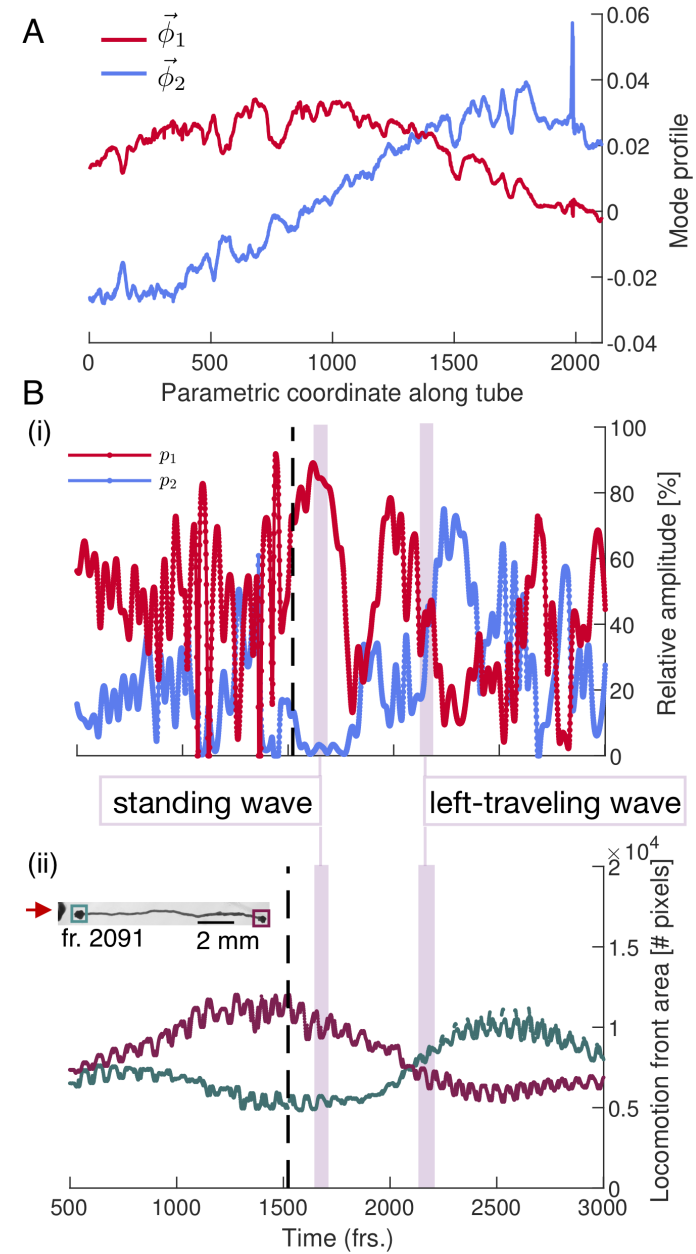

Fig. 5. Locomotion behavior of a single tube is determined by activation and temporal coupling of modes. (A) The two top-ranked modes. (B) (i) Activation of the two top-ranked modes given by their relative amplitude. (ii) Behavior of the locomotion front at each end of the tube over time. Vertical pink bars indicate two representative time intervals and the nature of the two-mode superposition is specified.

\section{Discussion}

To uncover the origin of behavior we quantified the dynamics of the often dubbed intelligent slime mold $P$. polycephalum. The simple build of this non-neural organism allows us to trace contractions of the acto-myosin-lined tubes, compute cytoplasmic flows from the contractions and finally link these dynamics to the emerging mass redistribution and wholeorganism locomotion behavior. Decomposing the contractions across the network into individual modes, we discover a large intrinsic variability in the number of significant modes over time along a continuous spectrum of modes. Triggering locomotion, we identify that states with few significant modes and regular contraction patterns correspond to specific behaviors like locomotion. Yet, also irregular contraction patterns consisting of a large number of significant modes are present, particularly marking the transitions between different regular states. Our findings suggest that a continuous spectrum of contraction modes allows the living matter network $P$. polycephalum to quickly transition between a multitude of behaviors using the superposition of multiple contraction patterns. 
Our observation of interlaced regular and irregular contraction patterns in $P$. polycephalum reminds of the strongly correlated or random firing patterns of neurons in higher organisms (3). Also in neural organisms, stereotyped behaviors are associated with controlled neural activity, as for example for locomotion in C. elegans (41) or the behavioral states of the fruit fly Drosophila melanogaster $(42,43)$. Though rarely discussed, variability in the dynamics of behavior is also observed in such neuronal organisms $(44,45)$. It is, thus, likely that the transition role of irregular states consisting of many significant modes observed here for $P$. polycephalum parallels the mechanisms of generating behavior in higher forms of life.

P. polycephalum is renowned for its ability to make informed decisions and navigate a complex environment (11-17, 46, 47). It would be fascinating to next follow the variability of contraction dynamics during more complex decision-making processes. Further, it would be interesting to observe 'idle' networks during foraging over tens of hours. It is likely that the contraction states with many significant modes here act as noise that can spontaneously cause the organism to 'decide' and reorient its direction of locomotion.

P. polycephalum's body-plan as a fluid-filled living network with emerging behavior finds its theoretical counterpart in theories for active flow networks developed recently $(48,49)$. Strikingly, these theories predict selective activation of thick tubes which we observe in the living network as well, prominently appearing among the top ranking modes, see $\vec{\phi}_{4}$ Fig. 1C(i) or $\vec{\phi}_{3}$ in Fig. 3C. This is a first hint that dynamics states arising from first principles in active flow networks could map onto behavioral and transition states observed here.

Likely our most broadly relevant finding in this work is that irregular dynamics, here arising in states with many significant modes, play an important role to switch between behaviors. This should inspire theoretical investigations to embrace irregularities rather than only highlighting regular dynamic states. The most powerful aspect of $P$. polycephalum as a model organism of behavior lies in the direct link between acto-myosin contractions, resulting cytoplasmic flows and emerging behaviors. Therefore, the broad understanding of the theory of active contractions (30-34) might well be the foundation to formulate the physics of behavior. This would not only open up an new perspective on life but also guide the design of bio-inspired soft robots with a behavioral repertoire comparable to higher organisms.

\section{Materials and Methods}

Experiments. The specimen was prepared from fused microplasmodia grown in a liquid culture (50) and plated on 1,5\%-agar. The network was trimmed and imaged in the bright field setting in Zeiss ZEN 2 imaging software with a Zeiss Axio Zoom V.16 microscope equipped with a Hamamatsu ORCA-Flash 4.0 digital camera and Zeiss PlanNeoFluar $1 \mathrm{x} / 0.25$ objective. The acquisition frame rate was 3 sec. The stimulus was applied in a form of a heat-killed HB101 bacterial pellet in close network proximity.
Distribution of temporal correlations. For a given time point $t_{i}$, the significant modes are determined based on the $70 \%$ criterion curve from Fig. 2A. Next, the temporal correlations among the coefficients are computed in a time interval of \pm 15 frames around the time point $t_{i}$. The correlations are then counted in bins of the appropriate row of Fig. 2A. Repeating this processing for all time points and normalizing each row by the total number of correlations in that row, we obtain the final distribution shown.

\section{ACKNOWLEDGEMENTS}

This work was supported by the Max Planck Society. In the final stages of this work P.F. was supported by the Simons Foundation program for Mathematical Modeling of Living Systems, grant number 400425. M.K. acknowledges the support of IMPRS for Physics of Biological and Complex Systems.

\section{Bibliography}

1. M. J. Angilletta and M. W. Sears, "Coordinating theoretical and empirical efforts to understand the linkages between organisms and environments," Integr. Comp. Biol. $\mathbf{5 1}$ no. 5, (2011) 653-661.

2. B. B. Wong and U. Candolin, "Behavioral responses to changing environments," Behav. Ecol. 26 no. 3, (2015) 665-673.

3. S. Marom and G. Shahaf, "Development, learning and memory in large random networks of cortical neurons: Lessons beyond anatomy," Q. Rev. Biophys. 35 no. 1, (2002) 63-87.

4. M. J. Jutras and E. A. Buffalo, "Synchronous neural activity and memory formation," Curr. Opin. Neurobiol. 20 no. 2, (2010) 150-155.

5. H. C. Berg and D. A. Brown, "Chemotaxis in escherichia coli analysed by three-dimensional tracking," Nature 239 (1972) 500-504.

6. R. H. Kessin, Dictyostelium: Evolution, Cell Biology, and the Development of Multicellularity. Developmental and Cell Biology Series. Cambridge University Press, 2001.

7. V. C. McMains, X.-H. Liao, and A. R. Kimmel, "Oscillatory signaling and network responses during the development of dictyostelium discoideum," Ageing Research Reviews 7 no. 3 , (2008) $234-248$. Pulsatility in Aging.

8. E. Ben-Jacob, O. Schochet, A. Tenenbaum, I. Cohen, A. Czirók, and T. Vicsek, "Generic modelling of cooperative growth patterns in bacterial colonies," Nature 368 no. 6466, (1994) 46-49.

9. E. Ben-Jacob, I. Cohen, and H. Levine, "Cooperative self-organization of microorganisms," Advances in Physics 49 no. 4, (2000) 395-554.

10. H. Sauer, Developmental Biology of Physarum. Developmental and Cell Biology Series. Cambridge University Press, 1982

11. T. Nakagaki, H. Yamada, and Á. Tóth, "Maze-solving by an amoeboid organism," Nature 407 (Sept., 2000) 470

12. A. Tero, S. Takagi, T. Saigusa, K. Ito, D. P. Bebber, M. D. Fricker, K. Yumiki, R. Kobayashi, and T. Nakagaki, "Rules for biologically inspired adaptive network design," Science $\mathbf{3 2 7}$ no. 5964, (2010) 439-442.

13. T. Nakagaki and R. D. Guy, "Intelligent behaviors of amoeboid movement based on complex dynamics of soft matter," Soft Matter 4 (2008) 57-67.

14. A. Dussutour, T. Latty, M. Beekman, and S. J. Simpson, "Amoeboid organism solves complex nutritional challenges," Proceedings of the National Academy of Sciences 107 no. $10,(2010)$ 4607-4611.

15. C. R. Reid, H. MacDonald, R. P. Mann, J. A. R. Marshall, T. Latty, and S. Garnier, "Decision-making without a brain: how an amoeboid organism solves the two-armed bandit," Journal of The Royal Society Interface 13 no. 119, (2016) 20160030

16. R. P. Boisseau, D. Vogel, and A. Dussutour, "Habituation in non-neural organisms: evidence from slime moulds," Proceedings of the Royal Society B: Biological Sciences 283 no. 1829, (2016) 20160446.

17. M. Aono, S.-J. Kim, M. Hara, and T. Munakata, "Amoeba-inspired tug-of-war algorithms for exploration-exploitation dilemma in extended bandit problem," Biosystems 117 (2014) 1 9.

18. Y. Yoshimoto and N. Kamiya, "Atp-and calcium-controlled contraction in a saponin model of physarum polycephalum," Cell Structure and Function 9 no. 2, (1984) 135-141.

19. T. Ueda, K. Matsumoto, T. Akitaya, and Y. Kobatake, "Spatial and temporal organization of intracellular adenine nucleotides and cyclic nucleotides in relation to rhythmic motility in physarum plasmodium," Experimental Cell Research 162 no. 2, (1986) 486 - 494.

20. N. Kamiya, R. D. Allen, and Y. Yoshimoto, "Dynamic organization of physarum plasmodium," Cell Motility 10 (1988) 107-116.

21. M. lima and T. Nakagaki, "Peristaltic transport and mixing of cytosol through the whole body of Physarum plasmodium," Mathematical Medicine and Biology: A Journal of the IMA 29 no. 3, $(07,2011) 263-281$.

22. K. Alim, G. Amselem, F. Peaudecerf, M. P. Brenner, and A. Pringle, "Random network peristalsis in Physarum polycephalum organizes fluid flows across an individual," Proceedings of the National Academy of Sciences 110 no. 33, (2013) 13306-13311.

23. K. Alim, N. Andrew, A. Pringle, and M. P. Brenner, "Mechanism of signal propagation in physarum polycephalum," Proceedings of the National Academy of Sciences 114 no. 20, (2017) $5136-5141$

24. J.-P. Rieu, H. Delanoë-Ayari, S. Takagi, Y. Tanaka, and T. Nakagaki, "Periodic traction in migrating large amoeba of physarum polycephalum," Journal of the Royal Society, Interface / the Royal Society 12 (05, 2015) .

25. O. Lewis, S. Zhang, R. Guy, and J. C. Del Alamo, "Coordination of contractility, adhesion 
and flow in migrating physarum amoebae," Journal of the Royal Society, Interface / the Royal Society $12(05,2015)$.

26. S. Zhang, R. D. Guy, J. C. Lasheras, and J. C. del Álamo, "Self-organized

mechano-chemical dynamics in amoeboid locomotion of Physarum fragments," Journal of Physics D: Applied Physics 50 no. 20, (Apr, 2017) 204004.

27. F. K. Bäuerle, S. Karpitschka, and K. Alim, "Living system adapts harmonics of peristaltic wave for cost-efficient optimization of pumping performance," Phys. Rev. Lett. 124 (Mar, 2020) 098102.

28. B. Rodiek, S. Takagi, T. Ueda, and M. J. B. Hauser, "Patterns of cell thickness oscillations during directional migration of Physarum polycephalum," Eur. Biophys. J. 44 (Apr., 2015) 349-358.

29. C. Oettmeier, K. Brix, and H. G. Döbereiner, "Physarum polycephalum - A new take on a classic model system," J. Phys. D. Appl. Phys. 50 no. 41, (2017) .

30. J. S. Bois, F. Jülicher, and S. W. Grill, "Pattern formation in active fluids," Phys. Rev. Lett. 106 (Jan, 2011) 028103.

31. M. Radszuweit, S. Alonso, H. Engel, and M. Bär, "Intracellular mechanochemical waves in an active poroelastic model," Phys. Rev. Lett. 110 (Mar, 2013) 138102.

32. M. Radszuweit, H. Engel, and M. Bär, "An active poroelastic model for mechanochemical patterns in protoplasmic droplets of physarum polycephalum," PLOS ONE 9 no. 6, (06, 2014) 1-12.

33. J.-D. Julien and K. Alim, "Oscillatory fluid flow drives scaling of contraction wave with system size," Proceedings of the National Academy of Sciences 115 no. 42, (2018) 10612-10617.

34. D. A. Kulawiak, J. Löber, M. Bär, and H. Engel, "Active poroelastic two-phase model for the motion of physarum microplasmodia," PLOS ONE 14 no. 8, (08, 2019) 1-19.

35. F. K. Bäuerle, M. Kramar, and K. Alim, "Spatial mapping reveals multi-step pattern of wound healing in physarum polycephalum," Journal of Physics D: Applied Physics $\mathbf{5 0}$ no. $43,(2017) 434005$.

36. G. J. Stephens, B. Johnson-Kerner, W. Bialek, and W. S. Ryu, "Dimensionality and Dynamics in the Behavior of C. elegans.," PLoS Computational Biology 4 no. 4, (2008) $1-10$.

37. D. Jordan, S. Kuehn, E. Katifori, and S. Leibler, "Behavioral diversity in microbes and low-dimensional phenotypic spaces," Proceedings of the National Academy of Sciences 110 no. 34, (2013) 14018-14023.

38. W. Gilpin, V. N. Prakash, and M. Prakash, "Vortex arrays and ciliary tangles underlie the feeding-swimming trade-off in starfish larvae," Nature Physics 13 no. 4, (Apr., 2017) 380-386.

39. N. Kamiya, "Physics and chemistry of protoplasmic streaming," Annual Review of Plant Physiology 11 no. 1, (1960) 323-340.

40. M. Li and J. Brasseur, "Non-steady transport in finite-length tubes," Journal of Fluid Mechanics $248(03,1993) 129-151$.

41. M. Liu, A. K. Sharma, J. W. Shaevitz, and A. M. Leifer, "Temporal processing and context dependency in Caenorhabditis elegans response to mechanosensation," eLife 7 (Jun, 2018) e36419.

42. G. Berman, D. Choi, W. Bialek, and J. Shaevitz, "Mapping the stereotyped behaviour of free moving fruit flies," Journal of The Royal Society Interface $11(08,2014) 20140672$.

43. G. J. Berman, W. Bialek, and J. W. Shaevitz, "Predictability and hierarchy in drosophila behavior," Proceedings of the National Academy of Sciences 113 no. 42, (2016) 11943-11948.

44. P. Grobstein, "Variability in behavior and the nervous system," Encyclopedia of Human Behavior 4 (1994) 447-458.

45. A. Renart and C. K. Machens, "Variability in neural activity and behavior," Current Opinion in Neurobiology 25 (2014) 211 - 220. Theoretical and computational neuroscience.

46. T. Ueda, M. Muratsugu, K. Kurihara, and Y. Kobatake, "Chemotaxis in Physarum polycephalum. Effects of chemicals on isometric tension of the plasmodial strand in relation to chemotactic movement," Exp. Cell Res. 100 no. 2, (1976) 337-344.

47. Y. Miyake, M. Yano, and H. Shimizu, "Relationship between endoplasmic and ectoplasmic oscillations during chemotaxis of Physarum polycephalum," Protoplasma 162 no. 2-3, (1991) 175-181.

48. F. G. Woodhouse, A. Forrow, J. B. Fawcett, and J. Dunkel, "Stochastic cycle selection in active flow networks," Proceedings of the National Academy of Sciences 113 no. 29 (2016) 8200-8205.

49. A. Forrow, F. G. Woodhouse, and J. Dunkel, "Mode selection in compressible active flow networks," Phys. Rev. Lett. 119 (Jul, 2017) 028102.

50. J. W. Daniel, J. Kelley, and H. P. Rusch, "Hematin-requiring plasmodial myxomycete.," J. Bacteriol 84 no. 5, (Nov, 1962) 1104-1110. 


\section{3 \\ Unravelling calcium dynamics in the plasmodial network}

Cell Signalling is AT THE BASIS OF ALL LIFE. In order to survive, cells need to constantly monitor the processes that are taking place inside them, as well as to respond to the changing 
environment. In the very early days of evolution, cells developed metabolic pathways that required efficient regulation of calcium concentration inside of the cell. Once the cells became highly sensitive and fast in response to the presence of calcium, the ground for cell signalling mechanisms was laid out ${ }^{\mathrm{I} 37}$.

Calcium is the ubiquitous signalling molecule. Cells use calcium to regulate every aspect of life; to trigger important events such as fertilization, apoptosis and differentiation, as well as to maintain their normal physiological state ${ }^{23}$. The signal transduction is typically initiated in two ways: by activating ion channels which are embedded in the membrane, or as a second messenger that is released after the stimulus is recognized, causing a string of chemical reactions called signalling cascades. Although they share a common ancestor, the mechanisms of calcium signalling are diverse and adapted to specific microchemistry of individual organisms ${ }^{30}$. Thanks to its ability to bind a number of motor proteins and their binding partners, calcium also lies at the heart of cell motility, enabling contractions of muscle tissue, beating of cilia and flagella, cytoplasmic flows, microtubule dynamics, and formation of pseudopods ${ }^{30}$.

In the slime mould $P$. polycephalum, it is generally agreed that calcium regulates the contractions of the actomyosin sheet enveloping the tubes that build the network of its body. However, the mechanism of the regulation still poses a puzzle. The binding of calcium to actomyosin produces opposing effects in plants and animals, respectively inhibiting ${ }^{\mathrm{I26}}$ and enabling ${ }^{30}$ contractions. P. polycephalum is an ancient organism at the crossroads of the animal and plant kingdom ${ }^{43}$, hence the regulating mechanism cannot be hypothesized by analogy.

In this chapter, we present the development of the framework for studying calcium dynamics in $P$. polycephalum. Starting with an introduction to calcium signalling and actomyosin regulation, we delve deeper with an overview of the research on calcium dynamics 
in P. polycephalum and formulate the central question: is calcium the universal signal and key to integrating information in P. polycephalum? After dedicating a significant part of this chapter to the development of experimental methods for calcium imaging and quantification, we present the results of successful imaging of calcium in a living specimen. In a series of experiments, we show the calcium response of the organism to mechanical perturbation and the effects of application of various chemicals associated with calcium dynamics in vitro and in other organisms. Lastly, we discuss the obtained results and provide an outlook for possible research in the future.

\section{I Calcium as a ubiguitous Signalling molecule}

In the stormy, bitter waters of the primordial soup, single cells started to use phosphate-based compounds in their metabolic pathways. The product of calcium ions and the orthophosphate group is weakly soluble, compelling the cells to develop ways of minimizing the calcium content in the cytoplasm. Consequently, the cells developed sophisticated machinery to keep calcium ions below the micromolar level of the ocean ${ }^{137}$.

Over the next two billion years, multicellular life emerged. ${ }^{23}$ The cells, now set to cooperate, were able to use their sensitivity to calcium as a foundation for development of signalling pathways. ${ }^{137}$ It is interesting that despite magnesium's greater abundance in sea water, nature preferred calcium over magnesium for this role. A presumed reason is the coordination chemistry of calcium which makes it versatile for binding with a large number of biomolecules ${ }^{23}$.

In the cytoplasm of most cells, the concentration of free calcium ions is kept in the nanomolar range ${ }^{30}$. However, the total concentration of calcium is measured in the micromolar range, indicating the cell's preference to keep calcium bound to proteins, compartmentalized 
in organelles or excreted into the extracellular matrix ${ }^{23}$. With the help of adequate capture and release machinery, this sharp gradient between the bound and unbound calcium allows for rapid cell response using calcium signalling ${ }^{\mathrm{I} 26}$.

The multitude of processes regulated through calcium signalling all share a common basic mechanism: the signal is triggered by a cue that causes a release of free $\mathrm{Ca}^{2+}$ ions into the cytoplasm, which in turn further triggers $\mathrm{Ca}^{2+}$-activated processes. Once the processes have been carried out, ion pumps remove the free calcium from the cytosol to establish the prestimulus calcium concentration ${ }^{16}$.

Due to the ubiquity of calcium signaling and specific microchemisty of each cell type, calcium signalling pathways evolved in a vast and complex network involving a multitude of interacting elements and spatio-temporal regulation ${ }^{16}$. Although each aspect is fascinating in its own right, this enormous field of research by far extends the scope of this thesis. For that reason, a detailed introduction to calcium signalling mechanisms will be omitted. Instead, we will focus on the aspects of calcium regulation we deem relevant for understanding the processes in $P$. polycephalum.

\section{3.i.i Calcium Regulation of actomyosin}

One of the most widely known roles of calcium in sustaining life, i.e. its regulation of actomyosin function, was also the first finding in the era of research on calcium signalling. In I883, Sidney Ringer performed an experiment on rat hearts and noticed that the hearts beat when suspended in tap water, but the beating ceases when the medium is replaced by distilled water $^{\mathrm{I33}}$. Having subsequently identified calcium as the key component for the beating of heart muscle, Ringer laid out the foundation for research on calcium signalling. ${ }^{22}$. 
Calcium regulation of actomyosin likely stems from the early cells' response to wounding. The rupture of a cell membrane is succeeded by a rapid release of free $\mathrm{Ca}^{2+}$ ions which trigger wound healing cascades. However, the early cells could have developed a preventative mechanism which would trigger the calcium release- and thus healing- before the actual rupture occurs. The hypothesised mechanism would give rise to what is today recognized as stretch-activated calcium channels, and the increased sensitivity to stretching would be achieved by coupling them to actomyosin whose binding partners Rho and ROCK are directly involved in wound healing. That way, the ability of actomyosin to contract could counteract the stretching of the membrane and help maintain cell shape. ${ }^{20}$

Due to its ability to contract and flow, actomyosin classifies as an active material ${ }^{56}$. Its three basic building blocks: actin, myosin and calmodulin are ubiquitous across eukaryotic organisms $s^{35,143,47}$ and are involved in essentially every process in the life of a cell, e.g. cell shape, mechanics, division, and migration ${ }^{9}$.

Actin is a highly conserved family of proteins ${ }^{48}$ with the ability to exist in a monomeric state called G-actin, or associate the globular monomers of G-actin into a filamentous form called F-actin. The molecules of F-actin are polarized and can further cross-link, giving rise to structures such as meshworks and bundles ${ }^{9}$. Its binding partner, myosin, represents a large family of motor proteins found exclusively in eukaryotes ${ }^{143}$. Exploiting the abilities to bind actin and hydrolyze ATP, myosin generates force for physical movement along actin fibers ${ }^{6 \mathrm{I}}$. According to current classification, there are 35 to 37 classes of myosin within the myosin super family, grouped by their genetic similarity and the role they perform in living organisms. ${ }^{\mathrm{I} I \mathrm{I}}$. The majority of myosin proteins consists of three domains: head, tail and neck. The head domain hydrolyzes ATP and binds to actin, exerting force and causing movement 
along the actin fibers ${ }^{\mathrm{I3} \mathrm{I}}$. The tail domain connects to cargo transport, and the neck domain has a linker role, in addition to binding light chains, independent proteins that regulate the function of myosin ${ }^{38}$. Calmodulin is a small and versatile calcium-binding protein found in all eukaryotic cells. It plays a role in numerous cellular processes, one of them being the contraction of smooth muscle, where a calcium-bound calmodulin activates the myosin light chain kinase, which in turn phosphorylates the head of the myosin light chain ${ }^{156}$.

The first regulatory mechanism of calcium on actomyosin was found in skeletal myosin of animals, where calcium binds regulatory protein troponin on the thin filament of the muscle to enable contraction ${ }^{125,169}$. The signalling leading to calcium-driven actomyosin contraction differs in smooth and striated muscles, however in both cases calcium has an activatory role in generating contractions ${ }^{53,82}$.

Among the multitude of regulatory functions calcium performs in plants ${ }^{171}$, the effect of inhibiting actomyosin contraction has been observed in plants that exhibit cytoplasmic streaming ${ }^{50}$. In several plants exhibiting calcium-mediated actomyosin inhibition, calcium was observed to cause dissociation of calmodulin, therefore hindering actomyosin function ${ }^{50}$.

\section{3.i.2 CALCiUm Regulation OF CELl MigRation}

Cell migration is a complex process resulting from concerted interaction of cellular machinery. The stringent control cell exerts on calcium dynamics makes it a good candidate for such a highly coordinated process ${ }^{167}$.

In polarized cells, such as mammalian cells that migrate by interacting with the substrate through creation of focal adhesions, the building blocks of the adhesions- actin and myosin, as well as their binding partners such as Rac, Rho and Cdc42- have to be regulated in a pe- 
riodic fashion. Myosin is regulated by short pulses of $\mathrm{Ca}^{2+}$ that are presumably emitted by the endoplasmic reticulum of the cell. Simultaneously, a gradient of calcium concentration from the back to the front of the cell is present throughout the locomotion process ${ }^{167}$.

A simple eukaryote, social amoeba Dictyostelium discoideum closely related to Physarum polycephalum is able of locomotion and chemotaxis. In 1996, Yumura et al. showed that $D$. discoideum also exhibits a back-to-front gradient of intracellular calcium during chemotaxis, pointing out the analogy with mammalian cells ${ }^{176}$. However, four years later, Traynor et al. disproved those findings, re-opening the questions about the role of calcium in ameboid locomotion ${ }^{165}$

\section{I.3 MEASURING THE INTRACELLULAR CALCIUM}

Considering the importance of calcium dynamics in all life forms and its ubiquity in cellular processes, calcium labelling has been an important goal since early days of calcium research. The development of first fluorescent indicators in 1980 caused calcium research to soar. Consequently, the development of increasingly specific and refined calcium indicators ensued, resulting in today's multitude of available calcium-specific dyes.

Calcium indicators bind only $\mathrm{Ca}^{2+}$ ions, i.e. calcium not bound to cellular components and free to diffuse in the cytoplasm. Most of the cellular calcium content is buffered by binding to proteins and the concentration of free calcium ions is highly dependent on the cell type. Calcium indicators themselves buffer free calcium, prompting careful selection of the indicator and its concentration in order to maintain homeostatic conditions ${ }^{119}$.

In an extensive review article, Takahashi et al. provide an overview of indicators and techniques for measurement of intracellular calcium ${ }^{154}$, including the properties of Calcium 
Green and Oregon Green, dyes used in this work.

\subsection{Calcium dynamics in P. polycephalum}

The appeal of research on $P$. polycephalum extends far beyond its ability to build optimized networks. Hidden away in a nook of the phylogenetic tree but still eukaryotic, P. polycephalum is an exciting system for investigating the role of calcium in the regulation of the actomyosin contractions that generate shuttle flow in the network.

In the late I970s, as the research on actomyosin expanded with the discoveries of new myosin types, Kessler et al. isolated and characterised the actomyosin content in $P$. polycephalum, finding that it bears a significant resemblance to the vertebrate muscle myosin ${ }^{71}$. Drawing an analogy with the previously established finding of calcium's activating role of actomyosin contractions in skeletal muscle cells, the early researchers expected the same to be the case in P. polycephalum.

However, in a later study, Kessler et al. discovered that the myosin light chain of $P$. polycephalum has the ability to bind calcium, a property previously unidentified in skeletal muscle $\operatorname{myosin}^{70}$. At roughly the same time, Kohama et al. performed complementary procedures of $P$. polycephalum myosin purification and analysis and showed that the binding of calcium in fact inhibits the interaction between actin and myosin, effectively hindering the contractions $^{78,77}$.

The inhibitory effect of calcium on the contractions and consequently cytoplasmic streaming is not unheard of; plants such as Nitella and Chiara undergo cessation of the cytoplasmic streaming when exposed to elevated calcium levels ${ }^{68}$. However, the similarity of $P$. polycephalum myosin to the skeletal muscle myosin made this finding unexpected, sparking in- 
terest in the research community and brought forward the need for a new model of calcium regulation.

Interestingly, several studies targeting the same phenomenon obtained the opposite result, i.e. the actomyosin activity increased with the addition of $\mathrm{Ca}^{2+67,86}$. However, Kohama et al. argue that this result can be reproduced by using crude preparations of actomyosin that still contains ATP-pyrophosphohydrolase, a $\mathrm{Ca}^{2+}$-activatable enzyme. After sufficient purification of the actomyosin sample, the result reverses and an inhibitory effect is reproducibly observed 77 .

Over the next forty years, Kohama et al. performed numerous studies of calcium regulation of $P$. polycephalum actomyosin which confirmed the initially identified inhibitory effect. In a recent review article, Kohama summarizes their results together with the findings of complementary studies on structure and function of $P$. polycephalum actomyosin and its relationship with calcium. In the current proposed model of calcium-actomyosin interaction, myosin is active when phosphorylated, and binding of calcium to phosphorylated myosin at the calcium-binding light chain deactivates it ${ }^{76}$.

In addition to the in vitro studies outlined above, calcium dynamics has also been studied in vivo. Yoshiyama et al. used a calcium-specific fluorescent dye to measure the change in the concentration of $\mathrm{Ca}^{2+}$ during contractions of a plasmodial fragment. The concentration of $\mathrm{Ca}^{2+}$ oscillated periodically, peaking right after the maximum contraction of the plasmodium. This result is consistent with the inhibitory effect observed in vitro. Additionally, the authors report that the increase in $\mathrm{Ca}^{2+}$ concentration propagates through the entire plasmodial fragment in a wave-like manner ${ }^{175}$.

Zhang et al. expanded significantly on this study by parallel measurement of contractions, 
cytoplasmic flows, calcium concentration and traction forces in a freely migrating small plasmodium lacking network structure. They found that the oscillations of calcium concentration are in phase with tractions stresses, which agrees with earlier reports from Yoshiyama et $\mathrm{al}^{179}$. However, the model of $\mathrm{Ca}^{2+}$ oscillator by Smith and Saldana ${ }^{149}$ proposes a complex biochemical basis of contraction generation, prompting Zhang et al. to suggest caution when interpreting the seemingly simple measured relationship between calcium and cytoplasmic flows or traction stresses. Indeed, a model of contraction generation in P. polycephalum by Julien et al. ${ }^{63}$ from 2018 features a flow-transported actomyosin activator. The dynamics of the hypothesized activator matches the one of experimentally measured calcium, indicating a likely high degree of complexity underlying the process.

While the model of Smith and Saldana propose a model underpinned by a detailed network of biochemical reactions ${ }^{\mathrm{I} 49}$ later expanded by Radszuweit et al. to account for diffusion and advection by cytoplasmic flows ${ }^{123}$, the other family of models of contraction dynamics prioritize mechanical and hydrodynamical coupling over the regulatory role of calcium ${ }^{\mathrm{I} 6 \mathrm{I}}$. In their recent review, Oettmeier et al. argue that an optimal model of oscillations in $P$. polycephalum should include both the biochemical basis as well as the hydrodynamics and mechanics of the system ${ }^{116}$.

\subsection{Calcium in locomotion of Physarum polycephalum}

Despite several missing puzzle pieces in the full picture, it is generally agreed that the plasmodium actively utilizes its contractions for locomotion ${ }^{97}$. Unfortunately, almost all models of P. polycephalum contractions ignore the interaction of the plasmodium with the substrate, resulting in an inability to probe locomotive behaviour. 
The model of $P$. polycephalum contractility by Zhang et al. ${ }^{177}$ is one of the first to combine the mechanical properties of the plasmodium in a poro-elastic model with the adhesive forces between the plasmodium and the substrate. They show that the coordination between hydrodynamics of the streaming, contractions and substrate-plasmodium adhesion are enough to reproduce their experimental results on flow and traction stresses in a migrating plasmodium. However, the model does not explicitly include calcium dynamics, leaving the role of the following experimentally observed spatial calcium gradient unexplained.

In two related studies in I 992 and I 993 , Natsume et al. probed the spatio-temporal patterns of calcium in a specimen exhibiting chemotactic behaviour. They measured the concentration of calcium using a ratiometric $\mathrm{Ca}^{2+}$ dye in a small plasmodium lacking network structure. In addition to the oscillations in cytosolic calcium levels, they report a consistently elevated concentration of $\mathrm{Ca}^{2+}$ at the migrating front of the organism and a corresponding lower concentration at the back, making up a stable gradient of calcium concentration along the plasmodium ${ }^{65}$. In the follow-up study, they introduced a repulsive stimulus to the migrating front and observed the gradient reverse while a new migrating front was forming ${ }^{\text {109 }}$.

Matsumoto et al. ${ }^{97}$ used these observations to argue that this asymmetry in calcium baseline concentration is the driving force behind the plasmodium's directed motion. However, their proposed mechanism of propulsion necessitates a higher concentration of calcium at the back of the plasmodium, contrary to the results of Natsume et al. ${ }^{65,109}$. Furthermore, in the extensive study by Zhang et al. from $2017^{177}$, ratiometric imaging of calcium in a migrating microplasmodium with a clearly defined body axis does not reveal a presence of a spatial gradient of calcium. 


\subsubsection{The Central QUestion and Strategy of THIS WORK}

Virtually all studies on calcium regulation in P. polycephalum were performed either in vitro or in small plasmodial fragments. The distinctive feature of the organism, however, is the network of interconnected tubes. The behaviour of P. polycephalum often-labelled as intel-

ligent, e.g. maze solving ${ }^{105}$, building an optimized network ${ }^{164}$ or decision-making ${ }^{32,91,130}$ all stem from the ability of the organism to build a network. In a 2007 article, Shirakawa et al. report generation of morphological order in the network. Arguing that this is an ability beyond computation, they suggest the existence of an emergent property in the plasmodium, likely linked to the contraction dynamics ${ }^{\mathrm{I} 46}$.

Taking into consideration the indications of calcium's regulatory role in tube contractions and plasmodium-wide organization, studying its dynamics in the tubular network appears to be the next logical step. Given the existence of emergent properties in the network, it is reasonable to expect different calcium dynamics compared to non-tubular plasmodial fragments. The plasmodial network, albeit rapidly changing its morphology, possesses a few additional advantages over plasmodial fragments. The endoplasm in the network is better resolvable from the gel-like wall, which could play an important role in studying the possible coupling between calcium in the endoplasm and the ectoplasm. Furthermore, the thickness variations of the tubes are smaller than in plasmodial fragments, allowing a better signal quality in fluorescence imaging. Lastly, the shuttle flow in the tubular network is generally of higher magnitude, aiding the assessment of the importance of hydrodynamic effects in the generation of contractions.

Here, we lay the experimental groundwork for answering the central question: is calcium the universal messenger and key to integrating information in the plasmodial network? Specif- 
ically, we devise experimental protocols for live-imaging of calcium in a network of $P$. polycephalum that would subsequently allow for answering specific aspects of the central question.

First, we address the question of calcium distribution in the network. As outlined above, a calcium gradient has been reported in plasmodial fragments lacking network structure. Due to the high magnitudes of shuttle flow, it is not clear whether a calcium gradient can be sustained in the tubular network. Another interesting phenomenon could be hidden in pruning tubes. The plasmodial network is undergoing constant reorganization, with many tubes pruning away as the new tubes are formed at the front of the organism. The causes and mechanism of pruning remain largely unexplored, and as a first step in uncovering this phenomenon, we explore the localization and dynamics of calcium in pruning tubes.

Second, we investigate calcium's potential role as the universal messenger by applying external stimuli to the plasmodium. Reports of wounding-induced calcium release are present in many living organisms (as described in Chapter 4), prompting an analogous study in $P$. polycephalum. Given the plasmodium's distinct body parts, i.e. migrating fronts and tubes constituting the bulk of the network, the question of potential differences in wound healing in different body parts arises naturally.

By devising a protocol for simultaneous imaging of free calcium and tube diameter dynamics, as well as developing quantification protocols, we build a base for deciphering the relationship between tube contractions and calcium dynamics. Furthermore, by establishing protocols for microinjection of dyes in a living plasmodium and live-imaging of calcium in plasmodial networks, we lay the groundwork to study calcium dynamics in the networks of P. polycephalum. 


\subsection{EXPERIMENTAL SETUP}

The plasmodia used in the experiments were developed from sclerotia (Carolina Biological Supply) grown at room temperature $(22 \pm 2){ }^{\circ} \mathrm{C}$ on $1.5 \%$ agar plates and fed with autoclaved oat flakes (Kölln brand).

The imaging was carried out using Zeiss Axio Zoom V.I6 microscope with a Zeiss PlanNeoFluar 1x/0.25 objective and Hamamatsu ORCA-Flash 4.0 digital camera. Imagine aquisition was performed with Zeiss ZEN 2 (blue) software in bright-field setting for bright-field imaging.

For fluorescence imaging, an external HXP 200C lamp was used for excitation of the dyes and the emitted light was collected with Zeiss filter set $38 \mathrm{HE}$ (excitation $\lambda=(470 \pm 40) \mathrm{nm}$, emission $\lambda=(525 \pm 50) \mathrm{nm})$ for calcium-specific dyes and Zeiss filter set 63HE (excitation $\lambda=(572 \pm 25) \mathrm{nm}$, emission $\lambda=(629 \pm 62) \mathrm{nm})$ for the passive reference dye.

The microinjection of the fluorescent dyes was performed with Eppendorf ${ }^{\circledR}$ FemtoJet ${ }^{\circledR}{ }_{4} i$ microinjector and InjectMan ${ }_{4} 4$ micromanipulator, using Eppendorf®Femtotips glass microcapillaries. The microcapillaries were loaded with Eppendorf®Microloader pipette tips.

\subsection{Fluorescent Dyes}

A passive, dextran-bound reference dye Texas Red ${ }^{\mathrm{TM}}$ (Thermo Fisher Scientific, $3000 \mathrm{MW}$, neutral) was used to correct for volume changes taking place due to tube contractions. Free calcium was labelled using dextran-bound Calcium Green ${ }^{\mathrm{TM}_{-\mathrm{I}}}$ (Thermo Fisher Scientific, $3000 \mathrm{MW}$, potassium salt, anionic) and dextran -bound Oregon Green ${ }^{\mathrm{TM}} 488$ BAPTA-I (Thermo Fisher Scientific, roooo MW, potassium salt, anionic). Using dextran-bound dyes for both passive and active labelling produces comparable clustering and aggregation be- 
haviour of the dye in both channels, which is of vital importance for the assessment of calcium dynamics.

The dyes were dissolved in distilled water to the final concentration of $3.0 \mathrm{~mm}$ for Texas Red, $2.7 \mathrm{~mm}$ for Calcium Green and $0.01 \mathrm{~mm}$ for Oregon Green dye. Using calcium-free buffers as dye solvent has not shown to be advantageous over distilled water. A calciumspecific dye was mixed with the passive reference dye in I: I ratio. Due to unforeseeable spillages and dye bleaching, the total dye volume loaded in the microcapillary should not exceed $5 \mu \mathrm{L}$.

The above listed dyes are large, dextran-bound molecules that require microinjection in the organism. Membrane-permeable dyes, specifically Cal-520(Abcam) and Fluo-8 (Abcam) were tested as well. However, presumably due to the extracellular slime layer, the uptake of the dyes was insufficient and the resulting signal was poor. A significant advantage of using dextran-bound dyes is prevention of dye compartmentalization due to the large size of the dextran molecule, which allows for extended imaging.

\subsubsection{Microinjection in Physarum polycephalum}

The success of the microinjection in $P$. polycephalum is largely dependent on two factors: suitability of the specimen and the microinjection technique. Well-fed organisms are most suitable for microinjection due to the optical and mechanical properties of their tubes. In a starving organism, the decreased transparency of the tubes presents a challenge to correct microcapillary positioning and injection, and the changed mechanical properties of the tubes often result in spillage and inability to close the injection wound. Furthermore, well-fed specimens are more likely to exhibit an increased hierarchy of tube diameters as well as a dense 
network of tubes, simultaneously providing thick tubes for microinjection and neighbouring tubes for efficient re-routing of the flow in case of tube obstruction. These properties combined significantly decrease the incidence of tube bursting, spillage and network-wide damage, consequently increasing the success of the injection.

The most successful microinjection technique tested in this work conquers the challenges posed by the mechanosensitivity of the tubes and makes use of the advective capabilities of the organism. A mechanical perturbation of the tube surface often results in cessation of the flows and gelation of the cytosol, rendering the tube unsuitable for injection. However, the effect of the mechanical stimulus introduced by the microinjection can be decreased with appropriate tube choice and microinjection technique. Ideally, the microinjection is performed on a tube of larger diameter $(>20 \mu \mathrm{m})$ that serves as a transport highway to the region of interest for calcium dynamics imaging. While it is possible to include the tube used for injection in the region of interest, it is inadvisable due to small dye spillages and wounding-induced calcium release even in best-performed microinjections. Even though a microinjection microcapillary with a smaller tip diameter introduces less mechanical perturbation, the choice of the microcapillary tip width needs to be balanced against the likelihood of microcapillary clogging. The thick extracellular slime will irrecoverably clog a narrow microcapillary as soon as the microcapillary touches the tube. A commercial narrow-tipped $(1 \mu \mathrm{m})$ microcapillary needs to be widened by breaking to $5 \mu \mathrm{m}$ at the tip for optimal results. This is best performed through a rapid movement of the microcapillary with its tip buried underneath the agar surface, or gently touching the sharp edge of a razor blade resting on the agar surface. A well-prepared microinjection microcapillary does not leak dye or release air bubbles.

A prepared microcapillary with the dye is positioned close to the plasmodial tube of choice. 


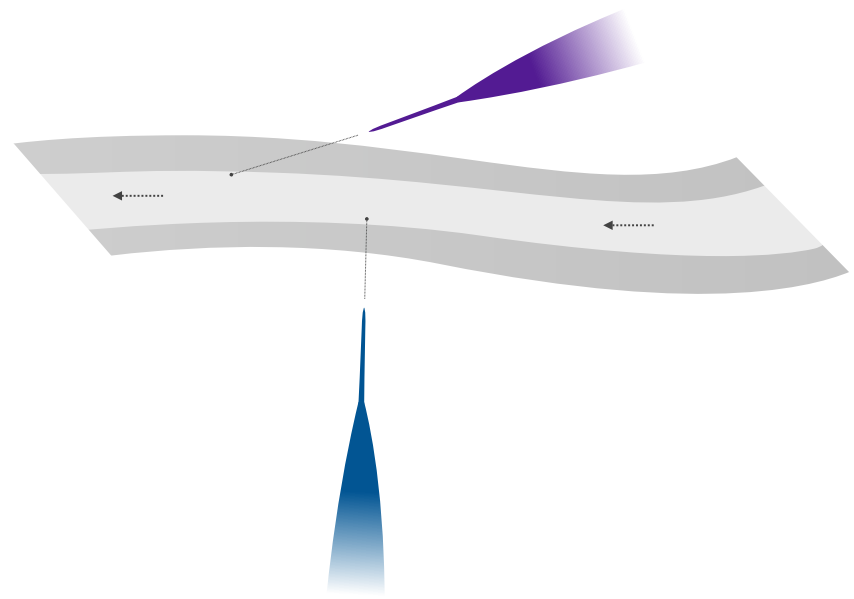

Figure 3.1: Microinjection in a tube of $P$. polycephalum: two microcapillary positioning approaches. The purple microcapillary on the upper side of the tube represents the acute angle approach and the blue microcapillary on the lower side of the tube represents the perpendicular approach. The movement of the microcapillary into the plasmodial tube is depicted by the dotted line extending from the capillary tip into the tub, and its end denotes the position for microinjection. The direction of protoplasmic flows is marked by dashed arrows.

Due to the organism's inherent sensitivity to light, the plasmodial network should be kept under constant illumination for at least $30 \mathrm{~min}$ to ensure a more stable morphology. The illustration in Fig. 3.I depicts two microinjection techniques that delivered best results in this work: perpendicular- and acute-angle approach. For both techniques, the direction of the protoplasmic flows is of crucial importance. The needle is placed in the immediate proximity of the tube wall and the microinjection is carried out once the flows are directed towards the region of interest. Preferably, the injection is carried out quickly and at the time of highest flow rate and the capillary is retracted quickly with as little lateral movement as possible. Furthermore, special care should be taken that the capillary does not obstruct the protoplasmic flows; in the best case, it will cause a cessation of the flows and bursting of the tube and 
spillage in the worst case. A plasmodium with a burst tube should be discarded due to dye spillage and the triggering network-wide wound healing signalling that will interfere with the measurement. The acute-angle approach is less likely to obstruct the flows, but more likely to mechanically perturb the tube wall compared to the perpendicular approach, for which the opposite is true.

Microinjection can be carried out in multiple sites in the network before imaging. It is advisable to trim away the injection sites before imaging, otherwise the continuous influx of the dye from the injection site combined with the inhomogeneity of the dye mixture due to wounding-induced calcium release could hamper the measurement of the homeostatic calcium dynamics. The imaging should start no earlier than $40 \mathrm{~min}$ after trimming to ensure the reorganization of the contractions induced by the trimming has taken place ${ }^{\mathrm{II}}$.

\subsubsection{Dye Calibration AND Bleaching}

The micromolar concentrations of the dyes used in the experiments might seem too high for the free calcium in nanomolar range, but the microinjected volume is very small compared to the total volume of the protoplasm. The organism injected with dye shows no changes in contraction dynamics or tube morphology, indicating that the used concentrations are not toxic and the dye does not buffer significant amounts of free calcium. Lower concentration of the dyes results in a weaker signal without an improvement in the overall health of the specimen compared to when higher concentrations are used.

Due to the inability to measure the injected volume, the dynamic nature of sol-gel conversion, the advection of the dyes, as well as their non-ratiometric properties, it is not possible to estimate the concentration of the dyes in the plasmodium. Furthermore, the dye particles 


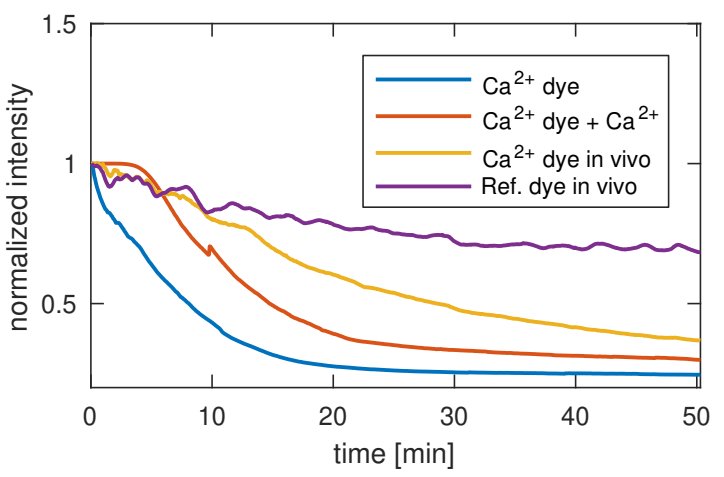

Figure 3.2: Bleaching rates of the calcium-specific dye differ in vivo and in vitro; reference dye bleaches at a lower rate than calcium-specific dye. In vitro: $10 \mu \mathrm{L}$ of $0.1 \mathrm{~mm}$ aqueous solution of Oregon Green dye alone (blue) and with $1 \mu \mathrm{L}$ of aqueous $1 \mu \mathrm{M} \mathrm{CaCl}_{2}$ solution (red). In plasmodium: total intensity of the plasmodium in calcium channel with Oregon Green dye (yellow) and reference dye Texas Red (purple).

tend to cluster and get trapped in vesicles, making the signal they produce unreliable. However, the homogeneous signal from the dyes in the sol and the comparable dextran-based structure of the calcium-specific and the reference dye enable the measurement of free calcium dynamics.

To investigate the bleaching behaviour of the dyes, the solutions of the dyes without calcium, as well as dyes mixed with solutions of calcium chloride in a range of concentrations from nanomolar to milimolar were imaged. The passive reference dye generally bleaches at a lower rate compared to calcium-specific dyes (Fig. 3.2). A universal calibration of the dyes is not possible due to the difference in bleaching dynamics in the organism compared to a water solution of the dye or its mixture with calcium chloride. 


\subsection{Results}

\subsection{DYE PROPERTIES SET THE EXPERIMENTAL CONSTRAINTS}

A successful microinjection results in a uniform spread of the dye in the plasmodium, as depicted in Fig. 3.3. Ideally, the imaging region of interest is positioned far from the microinjection site to minimize tube damage and allow for dye advection by shuttle flow. The latter results in an increase of the signal in both channels, however the dye incubation time before imaging should not exceed $45 \mathrm{~min}$ at most because the dye molecules are prone to aggregation and encasement in vesicles.

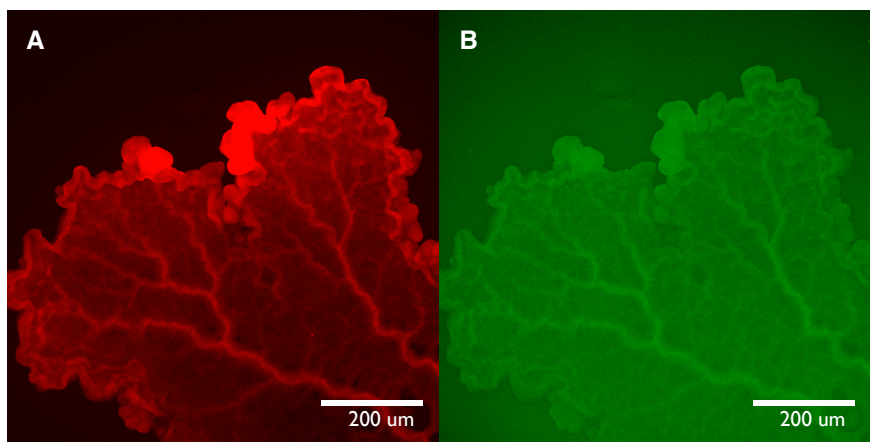

Figure 3.3: Distribution of reference dye Texas Red (A) and calcium-specific dye Calcium Green (B) in a growing plasmodial migration front.

The signal quality of the calcium-specific dyes is consistently lower compared to the one of the reference dye and decays more rapidly due to bleaching. Also, the autofluorescence of agar emitting in the same green part of the spectrum as the dye contributes to high background noise. However, these obstacles can be tackled to a good degree by using high concentrations of the dye, decreasing the exposure to fluorescent light and substituting agar with phytagel as plasmodium substrate to decrease background autofluorescence. 


\subsubsection{MeAsuring the CALCiUm GRAdient}

Measuring the hypothesized organism-wide calcium gradient is not expected to produce reliable results in the given experimental setup. Microinjection introduces tears in the tube wall that trigger wound repair signalling cascades, and the dye trapped in tube walls creates a gradient of signal from the injection site to the far end which cannot be avoided, even with distal microinjection sites. The short time window permitted by the tendency of the dyes to aggregate and get trapped in vacuoles does not allow for long incubation times that could potentially result in healing of microinjection or trimming sites. In order to reliably measure the calcium gradient, a membrane-permeable dye such as Cal-520 should be used. However, due to low signal and the tendency of such dyes to compartmentalize, caution is advised.

\subsubsection{EleVated CALCiUM Signal UPON THE APPLICATION OF MECHANICAL AND CHEM- ICAL STIMULUS}

To ensure the dye concentration is in a functional range, i.e. not too dilute thus producing weak signal nor too concentrated to buffer all free calcium, we applied two kinds of stimuli known to cause a change in calcium levels.

First, we applied a droplet of aqueous $0.2 \mathrm{~mm}$ EDTA solution on a section of the plasmodial tube (Fig. 3.4). EDTA, being a potent calcium chelator, rapidly caused a disruption of flows in the tube. The release and subsequent encasement of stored calcium can be observed as a short, bright calcium signal that fades out quickly $(<20 \mathrm{sec})$ as calcium is chelated. The absence of the same signal in bright-field and reference channel confirms that our choice of calcium dye and the concentration is suitable for investigating calcium-related phenomena in the plasmodial network. 

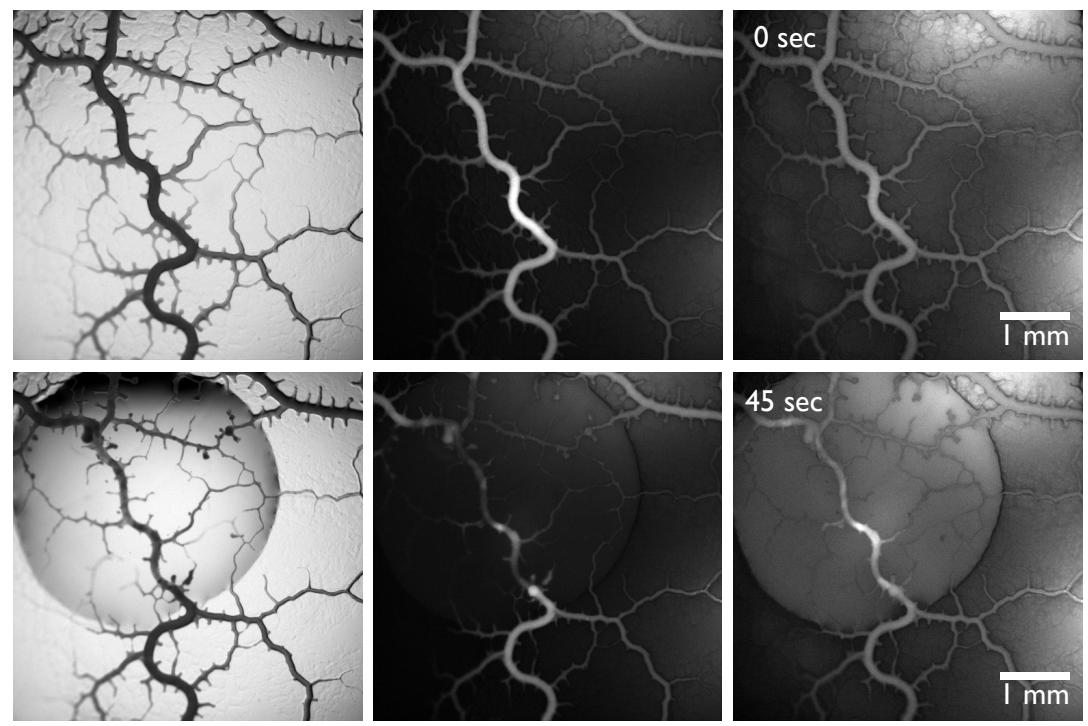

Figure 3.4: The effect of $0.2 \mathrm{~mm}$ EDTA solution on the plasmodial tube in bright-field channel (first column), fluorescent reference dye channel (middle column) and fluorescent calcium dye channel (last column). The application of the chemical causes a cessation of flows and a brief elevation of calcium-channel signal before its attenuation as calcium is likely released from storage and subsequently trapped by the chelator.

Second, in another plasmodium, we applied a quick mechanical stimulus by perturbing the tube wall with a microcapillary pipette tip. A burst of calcium signal lacking in the reference channel appeared right after the stimulation (Fig. 3.5) and faded as the tube wall healing took place, revealing that calcium is released upon wounding. The spatial distribution of the calcium signal that can be observed at the wounding site indicates the potential for highresolution tracking of the wound repair response in the future.

\subsubsection{INCREASE IN CALCIUM SIGNAL COINCIDES WITH WOUND HEALING}

We further probed calcium response in wound healing by mechanically perturbing a larger section of a rapidly growing migration front (Fig. 3.6). Apart from an increase of calcium signal that follows rapidly post-stimulus, pooling of body mass can be observed in the reference 


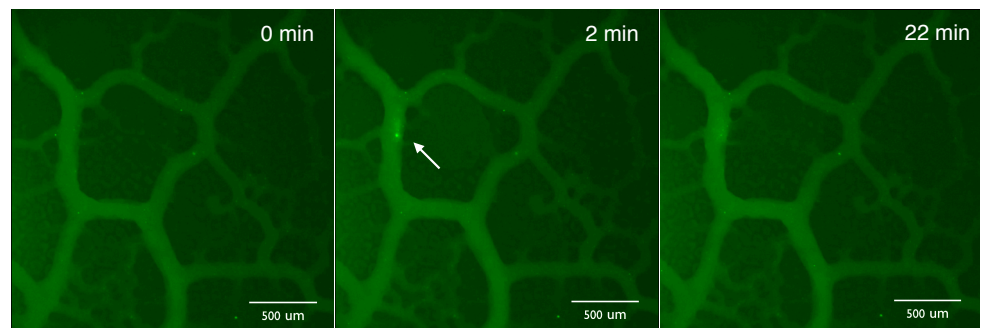

B
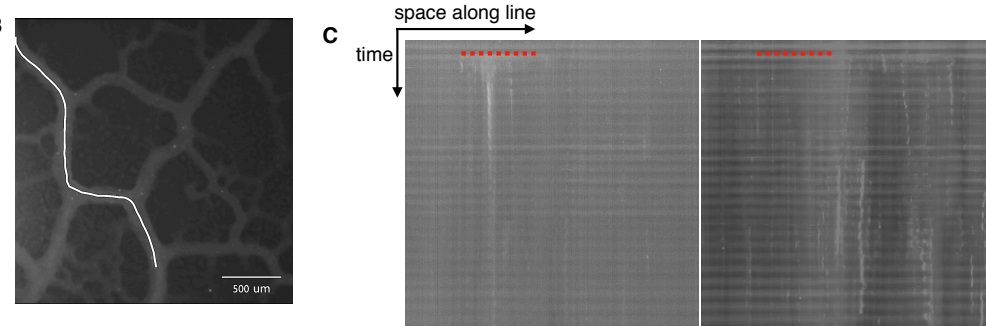

Figure 3.5: Mechanical stimulus causes an increase in calcium levels. (A) A quick mechanical stimulus was applied to the tube wall, causing an increase in calcium signal immediately visible at the site of stimulation (marked by white arrow) before fading away. (B) A kymograph was recorded along the tubes traced in white. (C) Kymograph along the traced tube in calcium channel (left) and reference dye channel (right). An increase in signal is clearly visible in the calcium channel while absent in the reference channel (marked by dashed red line).

and bright-field channel. This effect is a hallmark of wound healing in P. polycephalum (detailed in Chapter 4).

Strikingly, the areas of the migration front exhibiting pre-stimulus calcium levels, i.e. unaffected by the stimulus continued to grow at the same rate as before the stimulus, while the affected area lags. Given the presumed role of calcium in generating contractions and setting organism polarity, this phenomenon could be an indication of the fine-tuning of calcium signalling machinery in P. polycephalum, allowing the organism to use the same signal in a multitude of ways. 


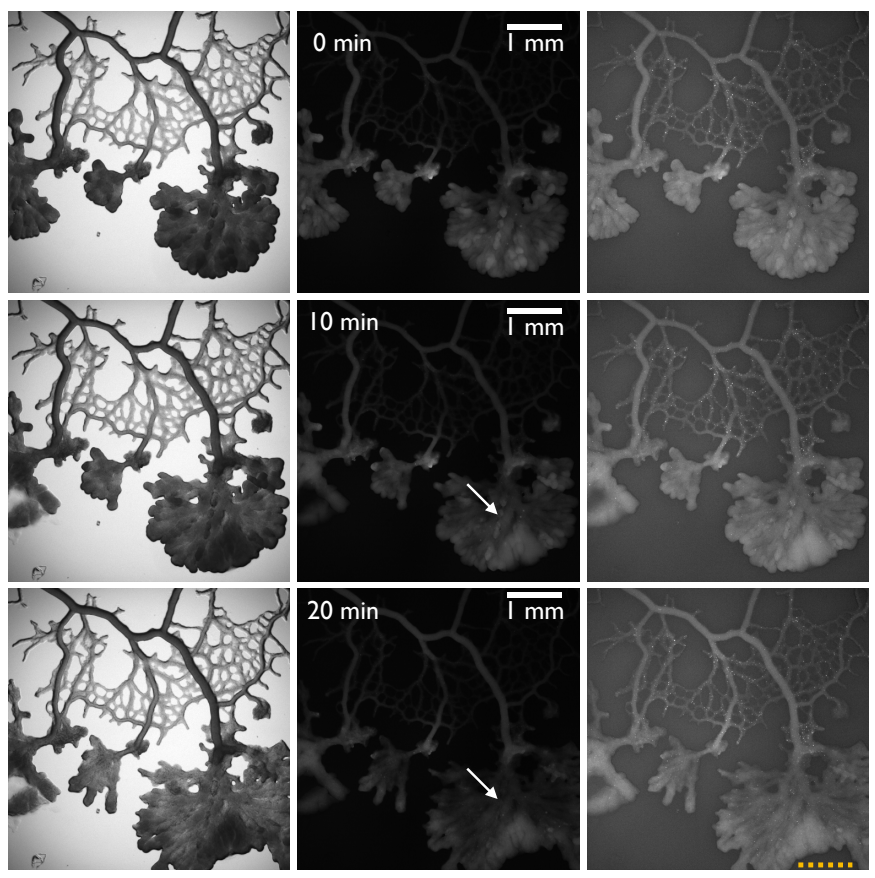

Figure 3.6: Mechanical stimulus applied to a growing migrating front causes an elevation in calcium levels and local stalling of growth. bright-field (first column), fluorescent reference dye channel (middle column) and fluorescent calcium dye channel (last column). A mechanical stimulus (marked by white arrow) causes a signal increase in the calcium channel and a stalling of fan growth at the site of elevated signal (denoted by yellow dashed line). Unaffected parts of the migrating front continue to grow at pre-stimulus rate.

\subsubsection{Pruning tube Deposits Calcium}

In a report by Kuroda et $\mathrm{al}^{85}$ from $\mathrm{i} 980$, the authors hypothesize that $P$. polycephalum regulates the calcium content during starvation by excreting calcium-containing vacuoles. We confirm this hypothesis by imaging a pruning tube in a functional plasmodium (Fig. 3.7).

The pruning of the tube starts with a gradual cessation of the flows through the tube. The tube subsequently undergoes a decrease in diameter in the middle section while the material is actively transported out into neighbouring tubes. Normalizing the signal in the calcium channel by the reference channel signal reveals a deposition of calcium in the pruned section of the tube. 


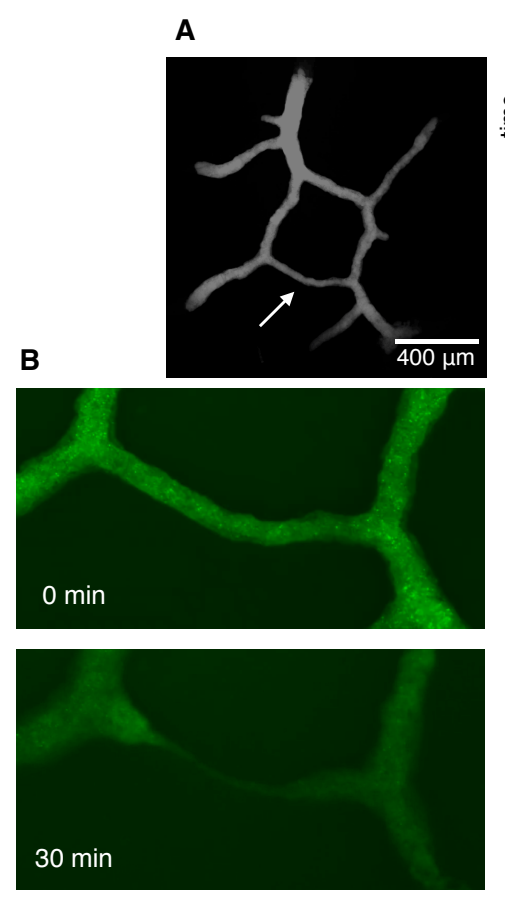

C

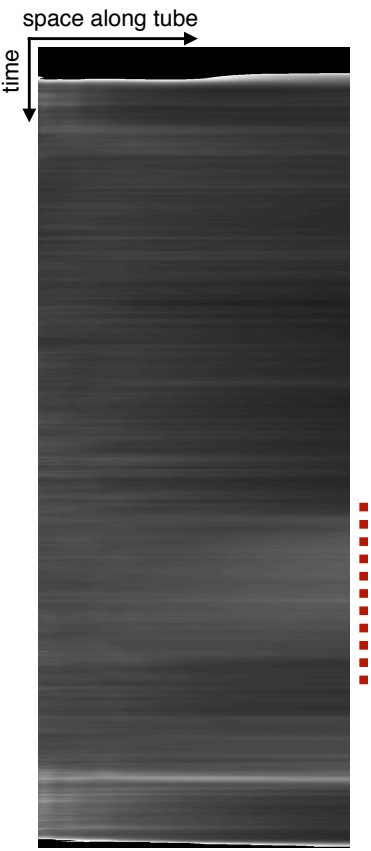

Figure 3.7: Calcium deposited by a pruning tube. (A) Position of the pruning tube in plasmodium marked by white arrow. (B) Time lapse of the pruning: functional tube at $0 \mathrm{~min}$ and cessation of flow through the tube due to pruning at $30 \mathrm{~min}$. Bleaching causes a decrease in the signal intensity at $30 \mathrm{~min}$ in the network bulk. (C) Kymograph along the length of the pruning tube. Calcium channel normalized by the reference dye channel. Dashed red line denotes the elevated calcium signal corresponding to the pruned segment.

\subsubsection{CALCiUm oscillations aRe in PHaSE With CONTRaCtions}

One of the long standing disputes regarding the role of calcium in P. polycephalum is its role in generating tube contractions. The current setup for measuring calcium dynamics consists of a calcium-specific dye channel and a passive reference dye channel that can be used to correct for volume changes caused by tube contractions. Due to the inability to precisely estimate injected volume in the imaging region of interest and the tendency of the dyes to aggregate, we deem measuring absolute concentrations of calcium from the signal unreliable even in the two-channel setting. However, the phase relationship between the time evolution of the 
A

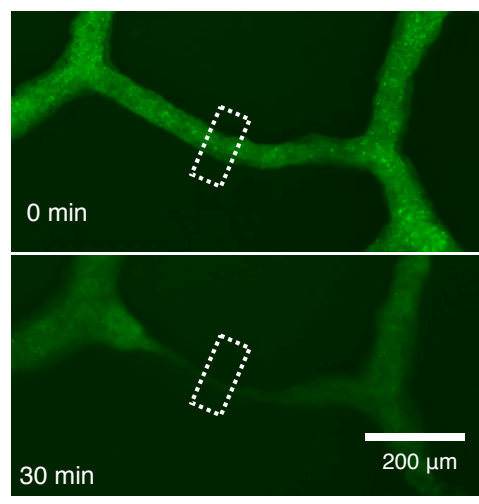

B

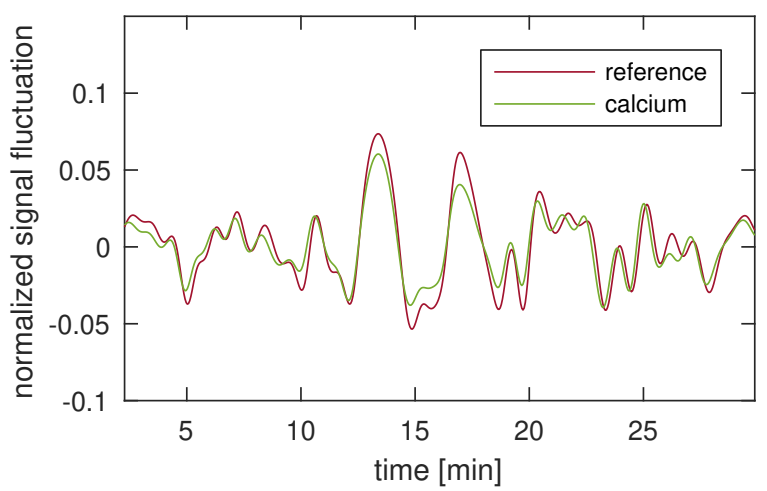

Figure 3.8: Oscillations of calcium are in phase with the contractions in the pruning tube. (A) Tracking of the time evolution of total signal in calcium-specific and reference channel within the region of interest denoted by dashed white rectangle. (B) Signal fluctuations over time for both channels. The signal is detrended to exclude the effect of bleaching. The fluctuations in both channels are comparable in magnitude and in phase.

signal from the two channels is unaffected by dye concentrations. Therefore, we expect the phase relationship to reflect the dynamics of calcium and tube contractions.

We first focus on the pruning tube discussed in the previous section and track the signal in a fragment of the contracting tube in the calcium-specific and reference channel over the course of pruning until the cessation of flows through the tube. The reasoning behind this choice is the expected distinctive contraction dynamics of the pruning tube compared to a sustained tube, potentially highlighting the role of calcium through previously unrecorded dynamics.

We track the cross-sectional oscillations of the tube within the marked region of interest in Fig. 3.8-A in calcium-specific and reference channel. The time series of signal fluctuation for both channels are depicted in Fig. 3.8-B. The fluctuations of the signals are matching in magnitude, however the calcium signal curve is shifted with respect to the reference signal, peaking $\approx 1.4 \mathrm{~s}$ earlier than the reference channel signal. 


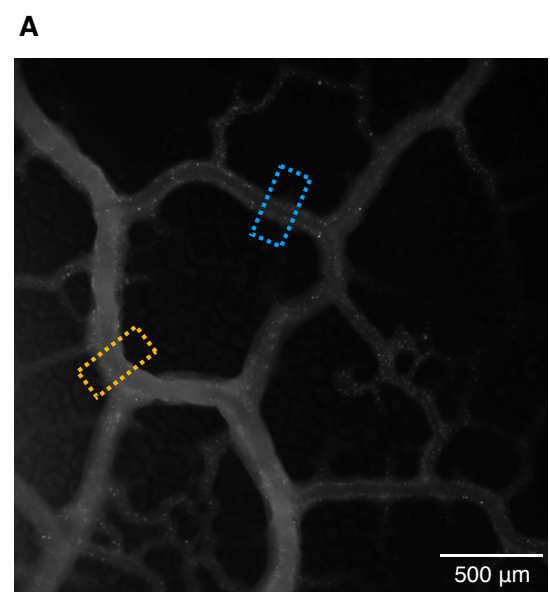

B

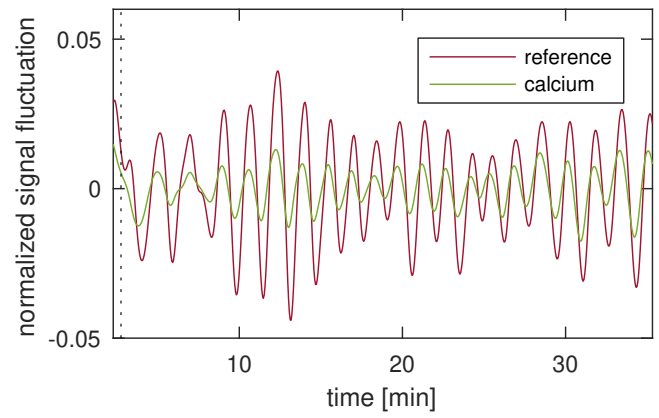

C

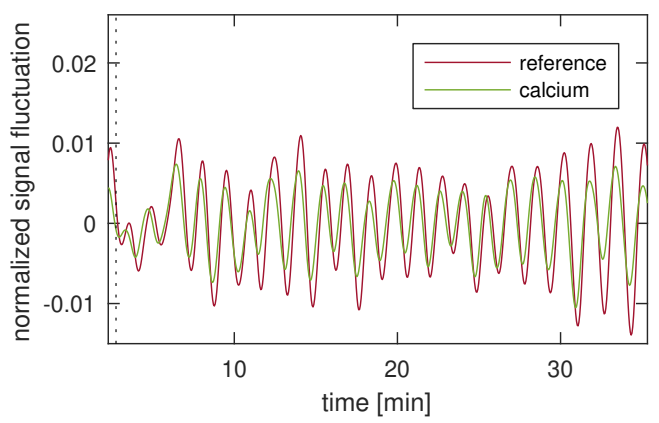

Figure 3.9: Relationship between calcium oscillations and contractions is consistent across different specimen and tubes, here a plasmodium with a mechanically stimulated tube shown previously. (A) Imaged section of a large plasmodium. Dashed orange rectangle denotes a tube section downstream of the mechanical stimulus, dashed blue rectangle denotes a tube unaffected by flow in the stimulated tube. (B) Fluctuation of the detrended signal in both channels in the tube segment marked in blue, stimulus time marked by dashed line. (C) Fluctuation of the detrended signal in both channels in the orange-marked tube segment, stimulus time marked by dashed line.

The same effect can be observed in the plasmodium introduced earlier, the signal fluctuations depicted in Fig. 3.9. Signal fluctuations were measured both in an unaffected and the mechanically perturbed tube. Even though the signal fluctuations in both channels temporarily diminish in the affected tube post-stimulus, the relative magnitude and phase lag of the fluctuations are consistent with the previous measurement.

A closer look into the imaging settings reveals the cause of the $\approx 1.4 \mathrm{~s}$ lag between the channels. The reference channel is recorded before the calcium-specific channel. With the acquisition time of both channels being $0.7 \mathrm{~s}$ and no delay between the channel acquisition within a frame, the expected lag of the calcium channel amounts to $1.4 \mathrm{~s}$. 
This result reveals that the signals of calcium-specific and the reference dye channel are actually in phase, indicating that the role calcium plays in regulating actomyosin could indeed be inhibitory, which is in agreement with the previous findings outlined above.

\subsection{Discussion}

By establishing protocols for imaging calcium in the plasmodial network, we paved the way for in-depth studies of calcium dynamics in the network and testing hypotheses posed by the theoretical models of $P$. polycephalum function. Most recent models featuring calcium dynamics such as the one by Schenz et al. ${ }^{142}$ from 2017 and Julien et al. ${ }^{63}$ from 2018 focus on adaptive tube formation and scaling of contraction waves, respectively. These are aspects of plasmodial function that necessitate the usage of tubes in experiments, i.e. plasmodial networks instead of microplasmodia.

The question of the existence of a calcium gradient along the migration axis is still a puzzle. While the work by Natsume et al. ${ }^{65,109}$ reports the existence of a gradient, later work by Yoshiyama et al. ${ }^{175}$ and Zhang et al. ${ }^{177}$ do not report the existence of a gradient despite their experimental setups being capable of measuring it. Especially in a network setting, the high flow rates in the tubes would likely cause the gradient in the tubes to flatten.

Due to its homogeneous build, resolving the calcium signal in the tube wall from the cytoplasm signal is challenging in a microplasmodium. By establishing a protocol for measuring the calcium signal at high resolution across the tube, we open the pathway to measuring calcium dynamics between the tube wall and cytoplasm, i.e. the key interacting components in a number of models of $P$. polycephalum function ${ }^{63,142}$.

However, for a model able to simultaneously emulate more than one calcium-involving 
phenomenon in the plasmodium at a time, an understanding of spatial and temporal decoding of calcium signal is imperative. As cells in virtually all organisms rely on calcium as the ubiquitous signalling species, the mechanisms to decode calcium signals have evolved to be very sophisticated $^{18, \mathrm{IOI}, \mathrm{I} 48}$. Investigating the decoding of spatio-temporal calcium signal in $P$. polycephalum would be an exciting pursuit, especially given the complexity of the organism's network, interconnectedness of body parts, and the high speeds of transport by flow. 


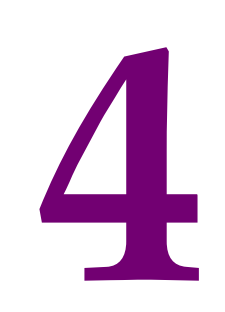

\section{Network-wide patterns of wound healing}

LIVING BEINGS ARE FRAGILE. Even animals covered in hard, armour-like keratin or chitin layers have unprotected body parts that can easily suffer damage. The ability to heal wounds is essential for survival and organisms have developed sophisticated mechanisms to control and undo the damage inflicted by mechanical forces. The response to wounding is preserved 
across living organisms and its intimate bond to all life sparked debate about it being the origin of consciousness ${ }^{8}$. Wounding starts with a disruption of the cell membrane. In order to protect the interior of the cell and prevent the influx of extracellular material, as well as cytoplasm loss, cells must be quick to minimise the damage. There are several proposed mechanisms of wound healing based on the observed processes of membrane hole closure in different organisms.

The first proposed mechanism features actomyosin contractions as key in plugging of membrane holes. Upon wounding, a contractile network of F-actin and myosin forms around the hole and pulls together the undamaged membrane and cytoskeleton surrounding the damage. However, the timescale of this process is at the order of minutes rather then seconds, potentially exposing the cell to the influx of unfavorable chemical species from the extracellular space ${ }^{107}$.

Another proposed mechanism is hole patching, where internal cell compartments are hypothesised to fuse at the site of damage, effectively patching the wound. Even though this mechanism can explain the rapid healing observed in several experiments, the identity of patch constituents is still unknown. Furthermore, patching would likely produce a double membrane which would have to be transformed into a single membrane through currently unknown mechanisms ${ }^{102}$.

Two interrelated possible mechanisms of wound closure are internalization and externalization; the first relying on endocytosis of the membrane fragment containing damage and the second facilitating wound closure by pinching off the damaged membrane. The existence of both mechanisms is supported by experimental evidence, but the size limit of membrane holes that can be repaired this way is likely small ${ }^{102}$. 
The quest of identifying the specific mechanism of wound healing is preceded by an important question: how does the cell know it has been wounded? In a clever evolutionary ploy, the cells learned to trigger healing upon influx of the same chemical species whose elevated levels lead to cell death: free calcium ${ }^{51,159}$. As described in more detail in the previous chapter, the cells exert stringent control over intracellular calcium levels. While an increase in cytosolic calcium sets off numerous signalling cascades, prolonged exposure of the cell interior to elevated calcium concentrations will cause precipitation of calcium phosphate and ultimately cell death ${ }^{\mathrm{I}}$.

Interestingly, despite the crucial importance of wound healing in maintaining cell function and preservation of parts of signalling cascades across eukaryotic kingdoms, the exact role of calcium influx in wound healing remains unexplained ${ }^{51}$. In a recent review article on the topic of cell wound healing, Nakamura et al. point out that the gradient of calcium concentration across the membrane necessary for wound healing is unusually high ${ }^{107}$, hinting that membrane potentials could play a key role in the healing process. Studying the dynamics of membrane potentials during cell wounding and repair in Xenopus laevis oocytes, Luxardi et al. ${ }^{94}$ showed that calcium influx causes an inward electric current at the center of the wound and an outward current at wound sides. The influx of positively charged calcium causes the membrane to depolymerize, triggering the repair processes. Surprisingly, they showed that calcium is essential throughout the whole process of would healing, not just the initiation; the external calcium helps maintaining the electric circuit, thus enabling cell membrane repolarization.

In Chapter 3 of this thesis, we showed that mechanical perturbation of a tube in the body of the slime mould $P$.polycephalum results in elevated levels of free calcium, likely stemming 
from the calcium-containing vesicles located in tube walls ${ }^{36}$. On the one hand, this result is hardly surprising and further demonstrates the ubiquity of calcium signalling in wound healing in eukaryotes ${ }^{5 \mathrm{I}}$. On the other hand, this finding is exciting because it opens the possibility of using $P$. polycephalum as a model organism for wound healing. The real appeal of studying wound healing in P. polycephalum, however, lies in the organism's intricate morphology and body-spanning periodic contractions. As a consequence of connectedness of the tubes in the plasmodial network, severing a single tube does not only perturb local function, but affects the whole plasmodium. Despite strand and network fragment excision being a routine step of specimen preparation in experiments, surprisingly little is known about wound healing in P. polycephalum. One consistent observation across previous studies is the gelation of the protoplasm induced by mechanical perturbation ${ }^{2}$. A cessation of the contractions and consequently flows has been observed, but unreliable to reproduce in the past experiments ${ }^{28}$.

This work is the first to address the impact of tube severing on the dynamics of the networkspanning contractions in P. polycephalum. We acquired time series of bright-field images of the plasmodium before and after tube severing and devised a method of mapping contraction dynamics onto a one-dimensional representation of the network. Reducing the complexity of the acquired information allows for identification of wound healing hallmarks in the contractile dynamics of the plasmodial network.

We found that the wound healing process in $P$. polycephalum is reflected in four successive phases of characteristic contraction dynamics. Right after wounding, the amplitude and frequency of contractions increases. This phase is followed by a network-wide stalling of contractions and flows. The two sub-networks that emerge as a result of severing restart the contractions, each network displaying an individual contractions dynamics. The re-fusing 
of the severed tube is marked by pooling of material in the form of a foraging front at the wounding site. As the severed tube regains function, the two sub-networks undergo vigorous, coordinated contractions. The vigorous contractions persist until the severed tube is fully recovered, and the network reverts to the pre-wounding contraction dynamics.

Our results show that wounding is a significant disruption of function of the whole network, even when the affected area is small compared to the total plasmodium area. Spatial mapping allows for extracting stereotypical contraction dynamics, which not only elucidated the previously debated stalling of contractions upon wounding, but revealed the step-wise wound repair response. Surprisingly, we found that the plasmodium treats wounding as an attractive stimulus and transports material to the cut site by creating a foraging front. This result shows the organism's intrinsic tendency to repair damages, rather than abandoning the wounding site by pruning away the damaged tubes. Also, the wounding reaction being markedly different to the reaction to light ${ }^{10}$, another unfavorable stimulus, showcases the fine-tuning of the organism to different environmental perturbations.

The questions that arise from the findings of this study inspire several directions of future research on $P$. polycephalum. First, the discovery of foraging front formation at the cut site indicates that the processes of wound healing and migration front establishment share a common molecular basis. Given the evidence of calcium release by wounding, a comparative study of calcium signalling in the two processes is a worthwhile pursuit. Furthermore, simultaneous labelling of actin or its binding partners and free calcium could provide a unique insight into cytoskeletal reorganization and clarify the still unsolved puzzle of internal organization of $P$. polycephalum.

The second intriguing direction for possible future research concerns the functioning of 
the network as a whole. While the terms robustness, resilience and optimization are regularly linked to P. polycephalum, the existing research mostly focuses on P. polycephalum's strategies of combating externally imposed threat or risk ${ }^{58,90,104}$. Studies on the dynamics of internal damage management and its impact on plasmodial network architecture and function are lacking. Research on that topic would be beneficial not only for gaining further insight into the function of $P$. polycephalum, but also as a model of other flow networks that occur in nature, such as vascular systems that often undergo internal damage and clogging of individual tubes and regions, leading to pathological states ${ }^{93}$.

The third potential direction of research deals with the coordination of contractions, a phenomenon unifying molecular machinery and network function. Even though the coordination of contractions in the $P$.polycephalum network has been assessed from different perspectives in this thesis (Chapters 2 and 3 ), the unique context of wound healing could prove advantageous over other settings for studying this phenomenon. If membrane potentials are indeed implicated in the organisation of contractions, measuring membrane potentials while disrupting the membrane by wounding would provide extraordinary insight both into the electrical activity of the plasmodium and its debated coupling to the actomyosin system.

Apart from uncovering another aspect of $P$. polycephalum function and opening alleys for future research, the results of this work have an important practical purpose. Trimming the plasmodial network in preparation for experiments carries the risk of studied phenomena being overshadowed by the organism's wound repair response. Our results showing that the response to wounding ceases after approximately $45 \mathrm{~min}$ allow for the execution of experiments without the risk of signal overlay. 


\title{
Spatial mapping reveals multi-step pattern of wound healing in Physarum polycephalum
}

\author{
Felix K Bäuerle ${ }^{1}$, Mirna Kramar ${ }^{1}$ and Karen Alim \\ Max Planck Institute for Dynamics and Self-Organization, D-37077 Göttingen, Germany \\ E-mail: karen.alim@ds.mpg.de
}

Received 31 March 2017, revised 31 July 2017

Accepted for publication 4 September 2017

Published 5 October 2017

\begin{abstract}
Wounding is a severe impairment of function, especially for an exposed organism like the network-forming true slime mould Physarum polycephalum. The tubular network making up the organism's body plan is entirely interconnected and shares a common cytoplasm. Oscillatory contractions of the enclosing tube walls drive the shuttle streaming of the cytoplasm. Cytoplasmic flows underlie the reorganization of the network for example by movement toward attractive stimuli or away from repellants. Here, we follow the reorganization of $P$. polycephalum networks after severe wounding. Spatial mapping of the contraction changes in response to wounding reveal a multi-step pattern. Phases of increased activity alternate with cessation of contractions and stalling of flows, giving rise to coordinated transport and growth at the severing site. Overall, severing surprisingly acts like an attractive stimulus enabling healing of severed tubes. The reproducible cessation of contractions arising during this wound-healing response may open up new venues to investigate the biochemical wiring underlying $P$. polycephalum's complex behaviours.
\end{abstract}

Keywords: slime mould, behaviour, transport network, image analysis

S Supplementary material for this article is available online

(Some figures may appear in colour only in the online journal)

\section{Introduction}

Simple organisms like fungi and slime moulds are able to display complex behaviours. This is surprising given that their network-like body plan lacks any central organizing centre. The slime mould Physarum polycephalum has emerged as a model system to study the complex dynamics these organisms use to adapt to their environment. The organism has been shown to find the shortest path through a maze [1] and connect food sources in an efficient and at the same time robust network comparable to man-made transport networks [2]. Furthermore, the slime mould distributes its body mass among several resources to obtain an optimal diet [3] and is able to anticipate recurring stimuli [4].

\footnotetext{
${ }^{1}$ Authors contributed equally to this work
}

P. polycephalum is a true slime mould that forms a plasmodial network. Nuclei keep on dividing without forming cell walls, which results in a syncytial web-like network. The cytoplasm within this tubular network flows back and forth in a shuttle flow [5]. These cytoplasmic flows are driven by cross-sectional contractions of the actin-myosin meshwork lining the gel-like tube walls [6]. Flows are organized across the entire network in a peristaltic wave of contractions that matches organism size [7]. Flows generated in the organism are optimized for transport as contractions increase the effective dispersion of particles way beyond molecular diffusivity by a mechanism called Taylor dispersion [8].

$P$. polycephalum adapts its network-like morphology to its environment by chemotaxis [9-11]. Here, stimulants are classified by being an attractant or a repellant depending on the organism's response to migrate toward or away from the stimulant. Stimulants have also been shown to affect 


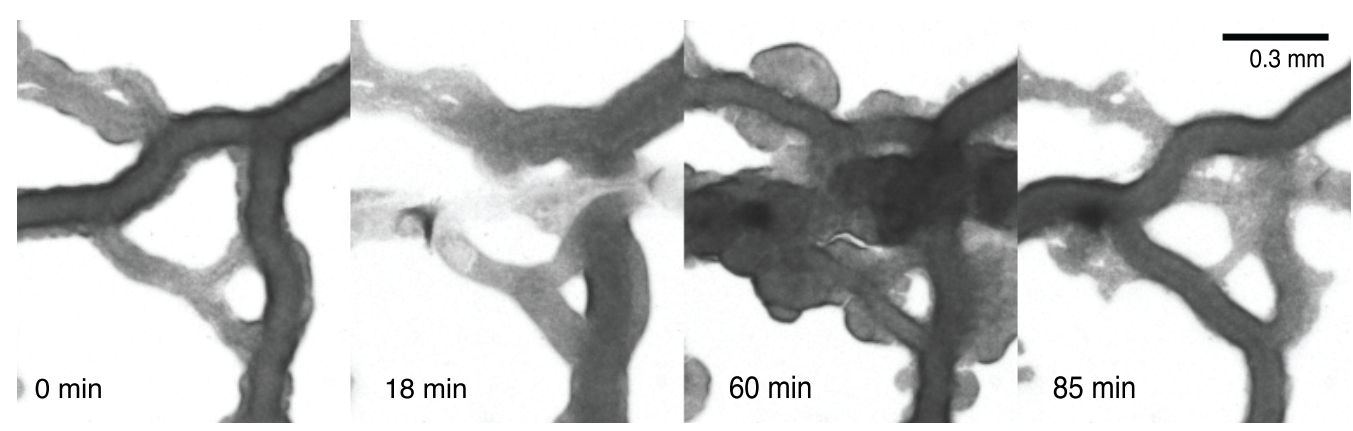

Figure 1. Wound healing process in $P$. polycephalum illustrated at four time points using bright field images. The cut occurred at 18 min and the fan grown at cut site reached its maximal size at $60 \mathrm{~min}$. The network morphology was restored after $85 \mathrm{~min}$.

cross-sectional contractions organism-wide by an increase in their frequency and amplitude for an attractant or a decrease for a repellant $[12,13]$. A variety of chemical stimuli have been discussed for $P$. polycephalum, with glucose being a prominent attractant and salts like $\mathrm{NaCl}$ being effective repellants [14-16]. Temperature [17, 18] and light [19, 20] have also been found to act as stimulants that trigger organismwide restructuring of the transport networks' morphology. In fact, the cytoplasmic flows themselves serve as the medium by which stimuli pervade the organism [21].

A lot less is known about the impact of mechanical perturbations on the organism. In its natural habitat the slime mould suffers predation from grazing invertebrates causing severing that disrupts the transport network and its cytoplasmic flows. In experiments it has been found that quickly stretching a strand to $10-20 \%$ of its length while keeping it intact increases the amplitude of oscillations [22]. Excising a single strand from a plasmodial network has been observed to lead to a roughly 20 minute cessation of contractions in the strand until recovery [23]. This phenomenon was not observed for strands excised from the growing fan region of the slime mould resulting in speculations about the motive force being limited to the fan only. Yet, the cessation of contractions turned out to be hard to reproduce, see [24] and references therein. Among these discordant observations what remains established is local gelation of cytoplasmic flows upon touch without severing the organism [25]. Despite the limited knowledge, wounding the organism by severing the network is part of daily laboratory routines and an eminent perturbation in natural habitat.

Here, we investigate $P$. polycephalum's dynamics during wound healing following the quick and complete severing of a tube within the organism's network. We follow the process of wound healing across the individual's entire body, over the course of one hour after severing. The exemplary quantitative analysis of organism-wide contractions reveals a stepwise response spanning four different states. Briefly after severing, the contractions are often marked by an increase in amplitude and frequency, followed by a several minutes long cessation of contractions and stalling of cytoplasmic flows. This resting state is terminated by a sudden restart of vigorous contractions as the severed tube re-fuses. The vigorous state then transitions into a state of network-spanning contractions and continuous fan growth at the wounding site until the organism reverts back to pre-stimulus dynamics. Timing and significance of individual steps varies with the severity of cutting and cutting site location within the network. For example, stalling is found to be less pronounced when the network is cut in fan-like region. Overall, quick and complete severing triggers a response pattern with characteristics of the response to an attractive stimulus, including an increase in amplitude and frequency and net movement to stimulus site, see figure 1. The reproducibility of stalling clarifies earlier contradictions and at the same time opens new avenues to investigate the biochemical dynamics behind the highly coordinated actomyosin contractions underlying $P$. polycephalum's arguably fascinating dynamics.

\section{Methods}

\subsection{Culturing and data acquisition}

The plasmodium is prepared from microplasmodia grown in liquid medium. The recipe for the medium is inspired by [26], see section $\mathrm{S} 1$. The advantage of this method over growing the plasmodium on oat flakes or bacteria is the ability to precisely control the nutritional state and amount of the organism. Also, plasmodia grown this way are free from oat flake residues or vacuoles containing food, which provides a cleaner sample for imaging. To prepare the plate for imaging, $0.2-0.5 \mathrm{ml}$ of the microplasmodia grown in a shaking culture at $30^{\circ} \mathrm{C}$ are transferred to an $1.5 \%$ agar plate and stored in a closed, but not sealed, dish in the dark. After $12-24 \mathrm{~h}$, the microplasmodia fuse into a single plasmodium. The plasmodium is ready for imaging when there are no visible traces of liquid medium and the organism assumed its characteristic network shape, which usually occurs up to $36 \mathrm{~h}$ after plating.

The imaging is performed with a Zeiss Axio Zoom V.16 microscope, equipped with a Zeiss PlanNeoFluar $1 x / 0.25$ objective and a Hamamatsu ORCA-Flash 4.0 digital camera. A green filter $(550 / 50 \mathrm{~nm})$ is placed over the transmission light source of the microscope to diminish $P$. polycephalum's response to the light, and a humidity chamber prevents the sample from drying out. The acquisition of the images is done in Zeiss ZEN 2 (Blue Edition) software with brightfield setting. During the acquisition, the illumination of the sample is kept constant, and an image is taken every $3 \mathrm{~s}$. The plasmodium is imaged for $\sim 1 \mathrm{~h}$ before the application of the mechanical stimulus to allow for the accommodation to the light [10]. The stimulus is applied manually, using a microinjection needle with a blunt tip. The needle tip is held above the 


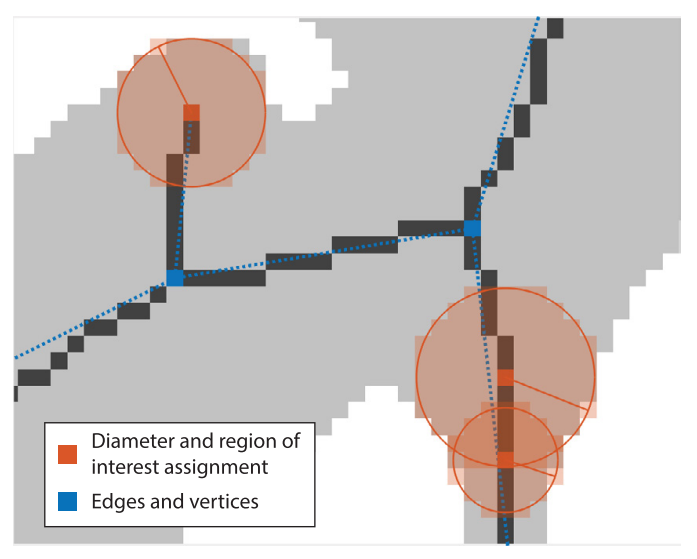

Figure 2. Scheme of intensity and diameter data extraction based on $P$. polycephalum bright field images. The light grey area depicts the network mask based on the bright field images. Dark grey lines represent the network skeleton and the corresponding topology is shown in blue. Each pixel of the skeleton acts as a reference point for data derived during the analysis. The diameter is set as the distance from the reference point to the next non-mask pixel. The intensity is calculated by averaging individual pixel intensities over a corresponding disk (red).

surface of the agar at a small angle and quickly dragged across the chosen plasmodial tube. The cut is severe and complete if the two parts of the tube separate completely. The plasmodium is then further imaged for more than $1 \mathrm{~h}$.

Using microplasmodia is so far the optimal way of obtaining non-severed networks, where the size and nutritional state are reproducible. However, there are challenges during the imaging that decrease the reproducibility of the experiment. In particular, plasmodia are highly motile and change their morphology accordingly. Furthermore, the organism tends to develop very large foraging fronts, which are not a suitable input for the presented comprehensive data analysis as they lack network characteristics. Lastly, the microscope light can act as stimulus $[2,19,20]$, and even the green-filtered lowintensity illumination may cause the network to respond and change its behaviour to escape the imaging region. These challenges combined make the reproducibility and required stability of the network morphology over time challenging.

\subsection{Comprehensive network-based contraction analysis}

To quantify contraction dynamics we analyse bright field recordings in two different ways: for two morphologically static networks (see E2 and E3 in the experiment list) we perform an exhaustive network-based analysis as outlined in the following (see figures 5 and S4, available online at stacks. iop.org/JPhysD/50/434005/mmedia). For the additional 19 specimen, which alter their network morphology dramatically over the course of the experiment, we analyse kymographs along static parts of the network as described in details in section S3 (see exemplary E1 and mov. S5).

Images recorded as a time series are processed as 8-bit uncompressed TIFs. At first every image is processed separately, then the results are stitched together, largely following [7], and lastly the collective is analysed. On every single image, background is removed with the rolling-ball method.

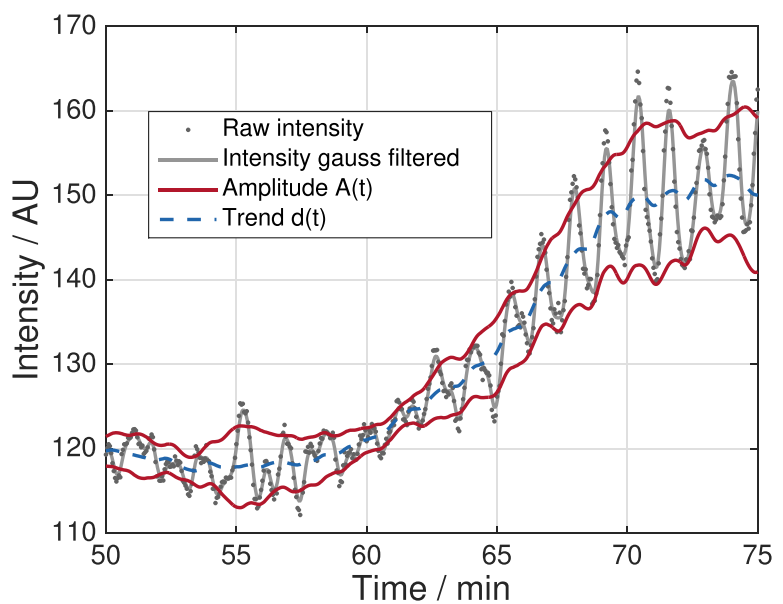

Figure 3. Derivation of oscillation specific parameters, i.e. amplitude $A(\mathrm{t})$, frequency $f(\mathrm{t})$ and trend $d(\mathrm{t})$, from single pixel time series. The trend is calculated using a moving average with a kernel width of 200 s. Intensity is filtered with a Gaussian of width 39 s. Amplitude and frequency are calculated from the absolute value and angle of the complex-valued analytic signal, respectively.

Then the image is used to create a mask, a binary image, with an intensity threshold that separates the network from the background. The mask is enhanced further, i.e. only the biggest structure is considered, small holes are filled and singlepixel edges are smoothed. Subsequently, the resulting mask is used as a template for extracting the network's skeleton with a thinning method. In the skeletonized mask each pixel can be understood as a data point representing local intensity and diameter (see figure 2). Local diameter is calculated as the largest fitting disk radius around the point within the mask. Within this disk the average intensity is computed and saved as intensity at the considered data point. Intensity and diameter anti-correlate due to the optical density of the slime mould and can therefore be used interchangeably considering BeerLambert law. Individual data points are attributed to a specific network branch of the network skeleton. To represent network topology, the network is broken down into vertices and edges where vertices describe pixel positions of branching points and edges represent two connected vertices. Each edge then acts as a parent for one specific branch. In this sense edges are abstracted simple connections and branches represent pixelbased resolution of a tube.

After the network is extracted in space, the edges, vertices, diameters, and intensities are concatenated in time. To map intensity and diameter over time, a reference image is used, usually from an early time point. For every data point the shortest distance to any pixel in the reference image is calculated. This gives a quasi-static $(x, y, t) \rightarrow$ (intensity, diameter) dataset, i.e. the topology and vertex positions stay the same, but intensity and diameter can vary. This is justified as long as growth of the organism and vertex movement is minimal. The oscillatory behaviour of tubes in a certain time window can be described by four time dependent variables, namely amplitude $A$, frequency $f$ (or period $P$ ), phase $\varphi$ and trend (base diameter) $d$. Each can be calculated from the time-evolution of the diameter or the intensity data, but if not stated otherwise the following results are only derived from intensity analysis. 

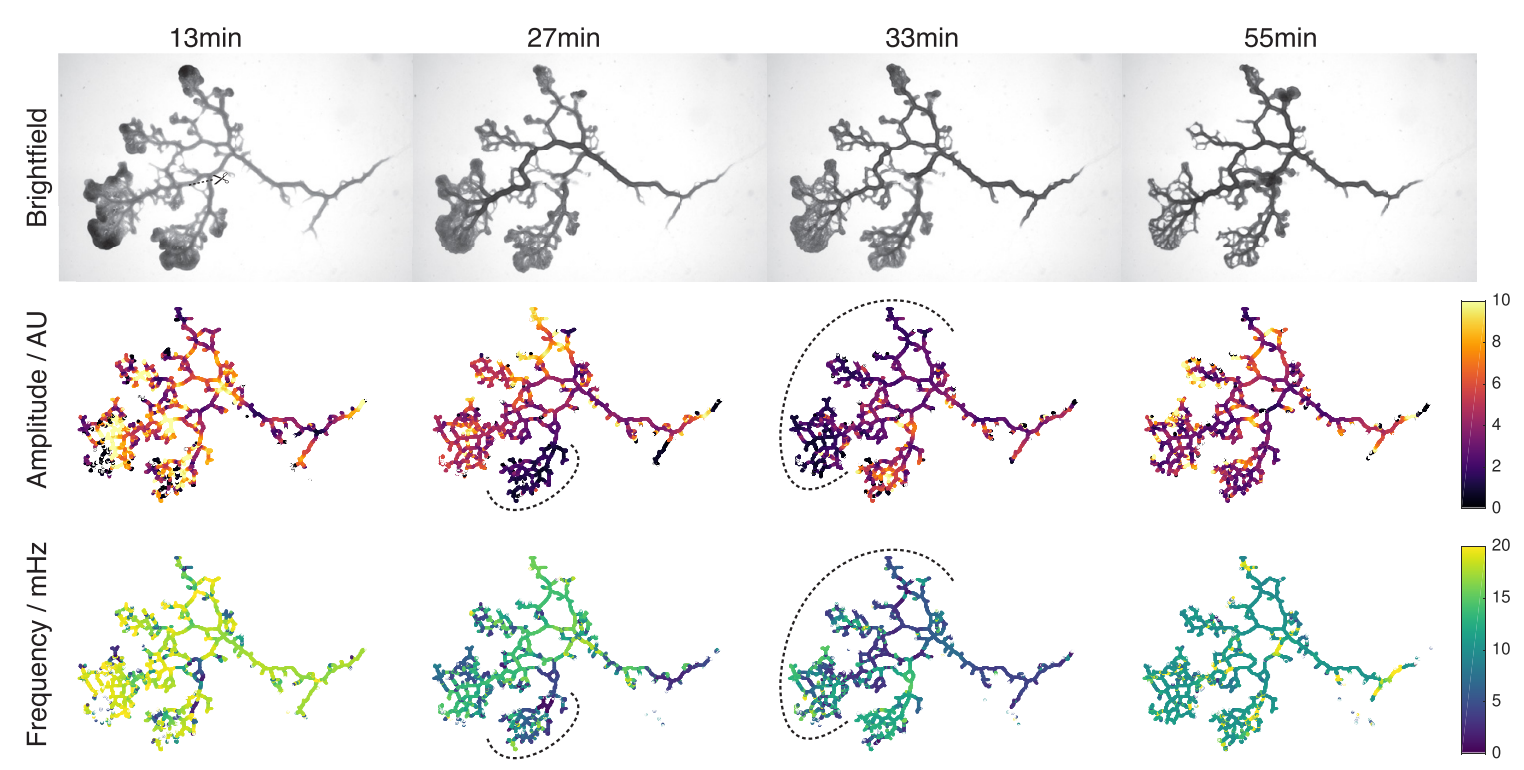

Figure 4. Time evolution of an exemplary network and its spatially mapped oscillation parameters at $13 \mathrm{~min}, 27 \mathrm{~min}, 33 \mathrm{~min}$ and $55 \mathrm{~min}$. The network was cut in the centre at 17.3 min (scissor icon). Top row depicts the raw bright field data, middle row the local amplitude, and bottom row the local frequency. Amplitude and frequency decrease locally, first at the lower sub-network (small dotted arc) at 27 min, subsequently at upper sub-network (large dotted $\operatorname{arc}$ ) at $33 \mathrm{~min}$. At $38 \mathrm{~min}$ cytoplasmic flows are re-established at the wounding site. Finally, amplitude and frequency values recover.

The trend $d(t)$ is obtained with a moving-average filter with a kernel width of $200 \mathrm{~s}$ on each time trace (see figure 3 ). The dataset is detrended using the calculated trend and smoothed with a Gaussian using a kernel width of 39s. The kernel widths were chosen to extract the characteristic contraction pattern which usually has a frequency of $\sim 90 \mathrm{~s}$. The values at every data point are stored as a complex valued time array, with the detrended and smoothed intensity representing the real part and the corresponding Hilbert transform representing the complex part, see S2 for more details. This time array, denoted analytic signal, serves as a basis to get instantaneous phase, frequency and amplitude by computing the angle or absolute value of the complex time series. Finally, the results are mapped back onto the network structure for each time point. In this fashion one can follow oscillatory behaviour resolved in time and space. Furthermore, the maps can be clustered in sub-networks and averaged separately to pinpoint local events in time. It should be mentioned that averaging of results for line plots, i.e. figure 5 , is always done after the data-point based analysis took place. In this way for example, the apparent amplitude of the averaged intensity (figure 5(D)) can be lower than the amplitude of each data point averaged (figure 5(B)).

\section{Results}

\subsection{Wounding induces fan growth at cut site}

We observe specimens before and after a quick and complete severing of a tube to follow the response of $P$. polycephalum to wounding (see figure 4, movs. S1 and S5). Bright field movies reveal that cutting of main tubes distal to fans triggers cessation of contractions followed by stalling of cytoplasmic flow ( $n=15$ out of 21). After contractions resume the severed tube fuses back together ( $n=21$ out of 21 ), i.e. flow is re-established, and a fan starts to grow at the cut site. Furthermore, we observe accumulation of body mass close to the cut site which is most prominent in peripheral cuts (figure S2). However, the growth is transient and after a given time the initial morphology is restored and the organism returns to typical behaviour comparable to before wounding.

In consideration of previously mentioned technical limits, we selected one representative dataset with prominent discernible features for network-based analysis. The following findings are derived from this dataset and later compared with other experiments. The specific timing of events in the representative data set is as follows (see figure 4). Two tubes are severed at $17.3 \mathrm{~min}$ effectively dividing the network into two parts. In both sub-networks, the size-wise bigger and smaller part, flows stall transiently around $30 \mathrm{~min}$. At $38 \mathrm{~min}$ a connecting tube is reinstated and starts to re-establish cytoplasmic flows across the cut site. Until about 63 min a transient fan is created at the cut site. At $90 \mathrm{~min}$ the initial morphology is restored and fans are grown elsewhere.

\subsection{Spatial mapping reveals localized stalling}

We perform network-based analysis on the wounded specimen to extract the interplay of contractions during the healing response. In particular, we map out the amplitude and frequency of contractions spatially (see figure 4, movs. S2 and S3). This allows us to exactly localize the onset of stalling as it goes hand in hand with low values of amplitude and frequency. Likewise, patterns in contraction dynamics in a region of interest are identified by spatially averaging amplitude and frequency in this region (see figure 5).

In the representative dataset, wounding separates the network into two sub-networks. Spatial mapping reveals that 

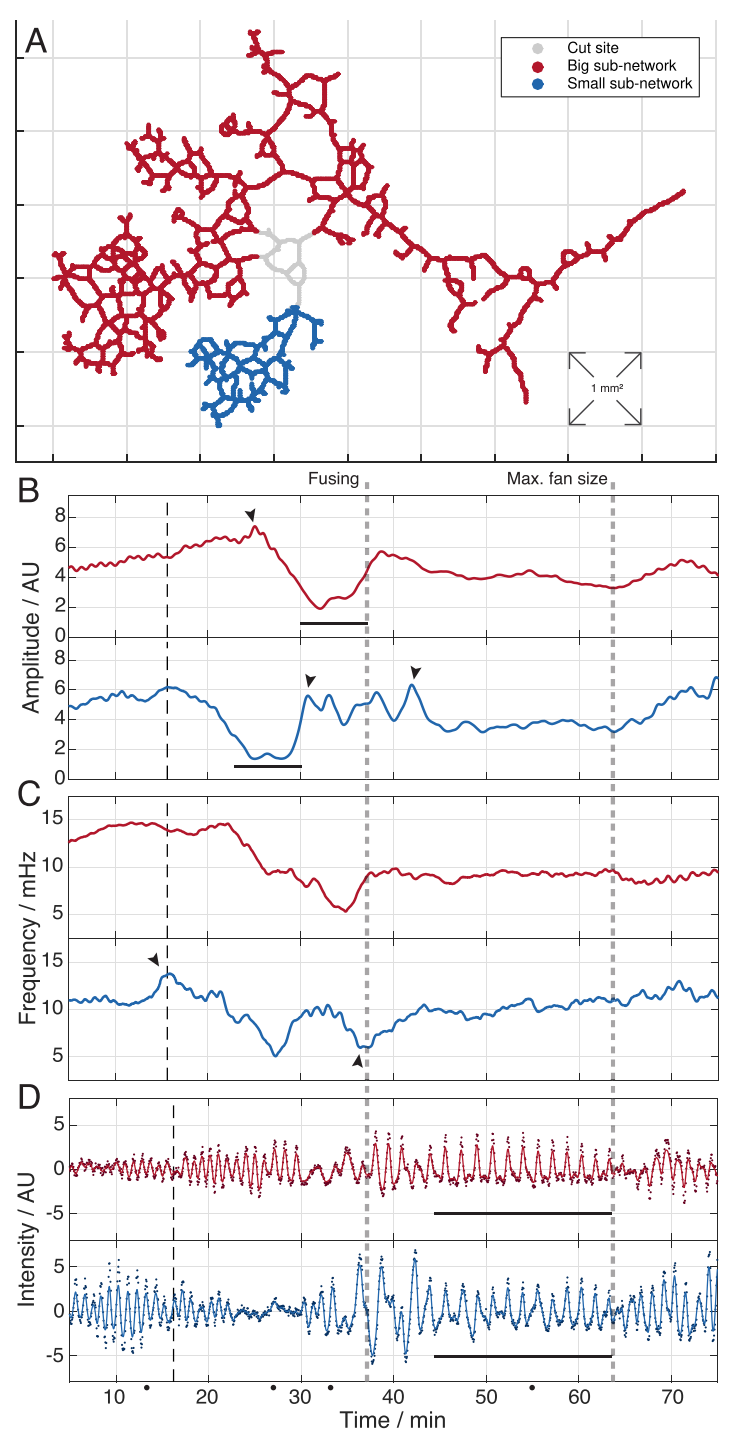

Figure 5. Comparison of oscillation parameters in the big- and small-sub-network depicted in (A) which result from the cut. Grey area (cut site) is not considered in the analysis. Time series of amplitude (B), frequency (C) and intensity (D) are averaged in the respective domains and compared; top: big sub-network, bottom: small sub-network. In each of these plots the black dashed line indicates the moment of the cut. The first grey dashed line marks the time point of fusion and the second the moment of maximal fan size. Black bars underline periods of stalling in (B) and fan growth in (D). In (D) the solid line represents the Gaussian filtered intensity (kernel width $=39 \mathrm{~s}$ ) and markers show raw averaged data. Black arrows indicate respective extremal peaks in the transition periods. Four black dots on the time line correspond to the four time points chosen in figure 4 .

oscillations cease on different time-scales in the two subnetworks. By identifying the two sub-networks as separate regions of interest, we quantify the patterns in contraction taking the spatial average of the respective contraction variables in each region. The small sub-network shows a drop in amplitude at $21.5 \mathrm{~min}$ by $63 \%$ and only recovers eight and a half minutes later to comparable values. Here, the percentage is given as ratio of time averages before, during and after stalling. In detail, the averages of the first $21.5 \mathrm{~min}$, the $9.5 \mathrm{~min}$ during stalling and $15 \mathrm{~min}$ after stalling were considered. The bigger sub-network drops significantly later at $28 \mathrm{~min}$ by $51 \%$ and recovers to $29 \%$ below the initial value nine minutes later. In the same time frames the frequency drops by $32 \%$ and $45 \%$ for the small and big sub-network, respectively. Yet, neither sub-network recovers its frequency fully right after the stalling phase. Only the small sub-network recovers 35 minutes later to initial frequencies whereas the bigger region levels off $35 \%$ below the initial value.

Furthermore, the phase patterns over time (see mov. S4) reveal changes in the travelling waves upon cutting. Initially ( 0 to $17.3 \mathrm{~min}$ ) one can observe peristaltic waves from the tail (right-hand side) to the front (left-hand side) which finally merge into concentric patterns in the fan regions. Then, at 18 to $30 \mathrm{~min}$, the small sub-network slows down noticeably (see change in frequency) and the big sub-network contracts with less apparent spatial correlation, i.e. the peristaltic wave pattern is temporarily lost.

\subsection{Fan growth phase coincides with stable network-spanning contractions}

After re-fusing of the two sub-networks, another distinct phase characterized by stable network-spanning contraction dynamics can be observed. In figure 5(D) contractions appear uniform from $44 \mathrm{~min}$ until $63 \mathrm{~min}$. During this phase, amplitude and frequency level off to a stable value with little fluctuations. The small sub-network shows a slight increase in frequency over this period and has more fluctuations in the average intensity data than the big sub-network. Note, that the time frame of these contractions coincide with fan formation at the cut site. Furthermore, the end of this phase also coincides with the largest fan in respect to area.

Network-spanning contractions are further supported by the phase time series. When considering the phase development one can already observe a peristaltic wave travelling towards the cut site in the small sub-network as early as $30 \mathrm{~min}$. A spanning pattern in the large sub-network is reinstated around the $35 \mathrm{~min}$ mark and a global pattern (small and large sub-network) appears roughly three minutes after re-fusing (40 min). Then a standing wave pattern appears between the central region including the cut site and the periphery. It is stable and network-spanning until $63 \mathrm{~min}$. Subsequently the phase pattern breaks into a peristaltic wave similar to pre-cut and propagates from the tail and the small sub-network into fan regions in the large sub-network.

\subsection{Stalling and fan growth periods are bridged by distinct transition periods}

Closer analysis of contraction dynamics over time reveals that the time point of the cut, the stalling phase and the fan growth phase are transitioned by phases of high fluctuations. Particularly in the presented case, before stalling occurs, amplitude and frequency peak shortly in both sub-networks (see arrows in figure 5). In the small sub-network this peak coincides with the cut, whereas another ten minutes pass for the big sub-network before the amplitude reaches its maximum. Surprisingly, here the frequency decline occurs three 
minutes before the amplitude drops. After stalling the amplitude increases sharply in both sub-networks, yet stays below previous values in the big sub-network. The small network undergoes a phase of roughly 13 min where the amplitude oscillates vigorously. This also coincides with a second frequency drop even though there is no apparent drop in amplitude at this time point. After the fan growth phase, amplitude and frequency show slight gradients once more. Here behaviour becomes comparable to the pre-cut state as the slime mould develops a preferred growth direction in the periphery and continues foraging.

\subsection{Fan creation and stalling is reproducible for complete severing}

For comparison we analysed a second dataset with the same network-based method (see figure S4). The key features, i.e. cut repair, stalling, a transition phase, stable network-spanning contractions and return to pre-cut behaviour, are found likewise, but the succession and timing of the specific events vary. This dataset has a weaker fan growth at the cut site and the time point of maximal fan size follows immediately after fusion. Given the short period of fan growth global network-spanning contractions are not observed. However, standing phase wave patterns are visible in the larger sub-network before fusion. Lastly, the transition phase shows peaking amplitude and fluctuating frequencies and reverberates for more than 30 minutes. At $70 \mathrm{~min}$ the network reinstates a peristaltic wave toward peripheral fan regions resuming pre-cut dynamics.

In further experiments analysed with a kymograph based approach, we confirmed stalling to be a common response after a cut (see figure E1, $n=15$ out of 21). However, the degree and duration of stalling is varying between experiments and is most reproducible for a severe cut close to the centre of the network.

In detail, we observe that both the degree and duration of stalling, depend on the network size and morphology, cut location, possibilities of re-routing the flow through neighbouring tubes and presence of large fans. Also, a network undergoing quick changes in morphology due to a presumed light shock is less likely to show stalling. Varying cut location shows that complete severing of a tube, with a diameter comparably large in size and few neighbouring tubes, results in strong stalling, see experiments $E[2,3,5,6,8,9,12,13$, and 18]. The effect is even more pronounced in smaller networks and on tubes close to the centre of the network (E[2, 3, 5, 8, and 18]). Stalling is less pronounced, as measured by relative change in amplitude and frequency as well as visual inspection of bright field data, if severing was applied to fan-like regions or peripheral tubes $(\mathrm{E}[10,11,14,15,16,17,19,20$, and 21]). If a severed tube had alternative routes with a comparable flow direction, neighbouring tubes inflated shortly after the cut, indicating a re-routing of flow. Yet, in this case stalling severity ranged from non-existent (E19) to full-stop (E1). In all data sets fan growth is observed around the cut site, yet duration and fan sized varied greatly (see E2 and E9 as maximal and minimal examples).

In all 15 experiments that show stalling, the period lasted for a minimum of three minutes. The exact time point of stalling onset and its duration varied. Duration of transition periods also varied from complete omission up to $22 \mathrm{~min}$ between cut and stalling. In 7 out of 15 experiments, a vigorous phase of increase in frequency or amplitude fluctuations could be observed in the transition phases.

\section{Discussion}

We investigate $P$. polycephalum's response to wounding in the form of a quick and complete severing of tubes using bright field microscopy and quantitative analysis of contraction patterns. Mapping out the contractions amplitude and frequency in space and time allows us to uncover a multi-step pattern of wound healing in $P$. polycephalum.

The key of our network-based analysis is mapping contraction variables onto a few pixels serving as the skeletonized backbone of the complete network. This representation allows us to capture contraction dynamics across the entire network over the course of several hours with handleable amount of data. Furthermore, spatial mapping visualizes abstract variables in an approachable way which outlines region of interests or patterns in space. For example, in the representative data set the time-shift in the response pattern between the two subdomains of the network would have been lost when averaging contraction dynamics across the entire network (see figure S1).

Among the multiple steps in the response to wounding the cessation of oscillations and stalling of the cytoplasmic streaming is most striking. The phenomenon of stalling of cytoplasmic flows has been observed previously [22, 23], but its reproducibility was deemed questionable [24]. Our work shows that cut location and severity are crucial parameters for inducing reproducible stalling. The stalling period is omitted when a tube is not completely severed, or cut in a way that allows the cut ends to rejoin quickly. In addition, the specific body plan affects the impact of a cutting stimulus. For example, severed fan-like regions show less pronounced stalling. However, we find reproducible strong stalling in networks where the affected tubes are crucial connections that cannot be re-routed easily - thereby clarifying previously discordant observations.

Stimuli are commonly classified into attractants or repellants. The response of $P$. polycephalum to an attractive stimulus includes fan growth and mass transport towards the stimulus site, often accompanied with an increase of oscillation frequency and amplitude. When we apply a wounding stimulus resulting in complete cutting of a tube, we observe a multistep response pattern where only two out of four steps show a noticeable increase in amplitude and frequency. Yet, wounding implies that the network architecture is perturbed. Taken into account that contraction frequency decreases as organism size decreases [27] the impairment of network architecture itself might counteract any increase in frequency. Despite the weak indication from contraction frequency and amplitude, we always observe fan growth and movement of mass toward the cut site regardless of the tube hierarchy, plasmodium size or the severity of the cut. Fan growth is a lot bigger than the initial spillage of cytoplasm due to cutting. Furthermore, we often identify a specific fan growth phase of network-spanning 
contractions well separated in time from the cutting event by the stalling phase. We therefore identify wounding as an attractive stimulus. The observation of network-spanning oscillations during fan outgrowth adds to our confidence about cutting being an attractive stimulus since the observed phase patterns resemble contraction patterns found in earlier work with attractive stimuli using glucose as a stimulant [21].

Employing spatial data analysis, we uncovered that wounding triggers a choreography of multiple successive steps to heal the severed tube. The mere duration of the healing response now defines a suggested minimal wait time after trimming for $P$. polycephalum experiments. The complexity of the response hints at an intricate signalling pattern underlying the coordination of contractions. It is likely that also the response to classical attractants and repellants, when scrutinized, reveal multiple steps. Unravelling the workings behind $P$. polycephalum's ability to adapt is arguably a fascinating albeit challenging question. Here, the reproducible cessation of contractions arising during this wound-healing response may open up new avenues to investigate the biochemical wiring underlying $P$. polycephalum's complex behaviours. Furthermore, it is fascinating that the impact of wounding can be weakened by network architecture. This suggests that $P$. polycephalum's body plan itself could be part of the organisms strategy to not only adapt to its environment, but also specifically prevent severe consequences of wounding.

\section{Acknowledgments}

We thank Christian Westendorf for instructions on growing microplasmodia, as well as for invaluable discussions and advice. MK and FB acknowledge support by the IMPRS for Physics of Biological and Complex Systems.

\section{References}

[1] Nakagaki T, Yamada H and Tóth A 2000 Nature 407470

[2] Tero A, Takagi S, Saigusa T, Ito K, Bebber D P, Fricker M D, Yumiki K, Kobayashi R and Nakagaki T 2010 Science 327 439-42
[3] Dussutour A, Latty T, Beekman M and Simpson S J 2010 Proc. Natl Acad. Sci. USA 107 4607-11

[4] Saigusa T, Tero A, Nakagaki T and Kuramoto Y 2008 Phys. Rev. Lett. 100018101

[5] Kamiya N 1981 Annu. Rev. Plant Physiol. 32 205-36

[6] Wohlfarth-Bottermann K E 1979 J. Exp. Biol. 81 15-32

[7] Alim K, Amselem G, Peaudecerf F, Brenner M P and Pringle A 2013 Proc. Natl Acad. Sci. USA 110 13306-11

[8] Marbach S, Alim K, Andrew N, Pringle A and Brenner M P 2016 Phys. Rev. Lett. $117178103-5$

[9] Ueda T, Muratsugu M, Kurihara K and Kobatake Y 1976 Exp. Cell Res. 100 337-44

[10] Durham A and Ridgway E 1976 J. Cell Biol. 69 218-23

[11] Chet I, Naveh A and Henis Y 1977 J. Gen. Microbiol. 102 145-8

[12] Miyake Y, Tada H, Yano M and Shimizu H 1994 Cell Struct. Funct. 19 363-70

[13] Hejnowicz Z and Wohlfarth-Bottermann K E 1980 Planta 150 144-52

[14] Kincaid R L and Mansour T E 1978 Exp. Cell Res. $116377-85$

[15] Hirose T, Ueda T and Kobatake Y 1982 J. Gen. Microbiol. 128 2647-51

[16] McClory A and Coote J G 1985 FEMS Microbiol. Lett. 26 195-200

[17] Matsumoto K, Ueda T and Kobatake Y 1988 J. Theor. Biol. 131 175-82

[18] Takamatsu A, Yamamoto T and Fujii T 2004 Bio Syst. $76133-40$

[19] Wohlfarth-Bottermann K E and Block I 1982 Cold Spring Harb. Symp. Quant. Biol. 46 563-8

[20] Nakagaki T, Yamada H and Ueda T 1999 Biophys. Chem. 82 23-8

[21] Alim K, Andrew N, Pringle A and Brenner M P 2017 Proc. Natl Acad. Sci. USA 1145136

[22] Kamiya N and Yoshimoto Y 1972 Aspects of Cellular and Molecular Physiology (Tokyo: University Tokyo Press) pp 167-89

[23] Yoshimoto Y and Kamiya N 1978 Protoplasma 95 123-33

[24] Cieślawska M and Kołodziejczyk J 1984 Cell Biol. Int. Rep. 8 813-21

[25] Achenbach U and Wohlfarth-Bottermann K E 1981 Planta $151574-83$

[26] Fessel A, Oettmeier C, Bernitt E, Gauthier N and Döbereiner H G 2012 Phys. Rev. Lett. 109078103

[27] Kuroda S, Takagi S, Nakagaki T and Ueda T 2015 J. Exp. Biol. 218 3729-38 


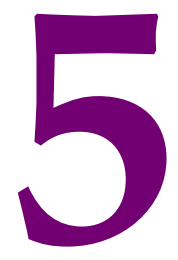

\section{Conclusion and Outlook}

The ABILITY Of THE SLIME MOULD TO SOLVE COMPLEX PROBLEMS WHILE RETAINING A SIMPLE BUILD EARNED IT THE ATTRIBUTES INTELLIGENT AND MYSTERIOUS. In the work presented in this thesis, we delved into the functional principles of Physarum polycephalum underlying a diverse range of observed phenomena. First, the study of the organ- 
ism's abilities to encode memory gave rise to previously unrecognized aspects of plasmodial network function. By identifying the hierarchy of tube diameters in the network as key in encoding and reading out the information about its environment, we discovered a strong reliance on internal organisation in the organism previously thought to be devoid of organizing centres (Chapter I). Next, breaking down the dynamics of the numerous contracting tubes of the plasmodial network revealed a multitude of oscillation modes present in the network at all times. The activity of the modes is highly dynamic and the organism relies on specific mode combinations to perform behavioural tasks, such as reaction to stimuli and locomotion (Chapter 2). Then, we addressed the role of calcium, the universal signalling species of immense importance in all living beings. By establishing experimental protocols for the measurement and quantification of calcium dynamics in the plasmodial network, we built a base for the study of phenomena in which calcium plays a significant role in $P$. polycephalum (Chapter 3). Lastly, studying the reorganization of the plasmodial network upon severe wounding revealed a multi-step pattern of contraction dynamics during the process of repair and re-establishment of network function. These results not only provide an insight into the wound healing process in $P$. polycephalum, but help increase the general accuracy and reproducibility in specimen preparation in experiments (Chapter 4).

While each of the studies presented in this thesis contributes to the understanding of $P$. polycephalum's function in their own right, just like the synergy of individual tubes giving rise to the intricate plasmodial behaviour, the most intriguing questions emerged as a consequence of the interaction between the studies that make up this thesis.

The first intriguing question threading through all studies presented here is the one concerning signal processing in $P$. polycephalum. The plasmodium navigates its environment 
with dexterity comparable to species possessing a complex nervous system, indicating the existence of sophisticated signal processing machinery. We found that the information about the nutrient stimulus is encoded by propagating a signal that softens the actomyosin cortex, but the dilation caused by softening is transient. Despite the continued presence of the nutrient source, we do not observe a flooding of the network by the signal that would ultimately lead to organism-wide softening and loss of contractility. The reason behind this phenomenon could lie in the dynamic threshold of the signal transduction network. In Dictyostelium discoideum, the close relative of $P$. polycephalum, changing the threshold of the signal transduction network alters cytoskeletal properties and allows the organism to switch between migratory modes ${ }^{100}$. Analogously, a dynamic adaptation of the signal threshold in the plasmodium would explain the retained consistency of the tubes while exploiting a food source, thus enabling foraging during feeding, as well as the ability to connect multiple food sources simultaneously ${ }^{32}$.

The role of the network hierarchy, another key concept that emerged in this work, appears to be key in signal processing in $P$. polycephalum. The plasmodium relies on cytoplasmic flows to spread the signal across the network. The flow velocities, being a function of tube diameters, directly depend on the tube hierarchy. Conversely, there is likely a feedback of the flows on the tube walls giving rise to long-term tube diameter dynamics reminiscent of flow-driven blood vessel pruning in the brain of the zebrafish Danio rerio ${ }^{26}$, another instance of a biological flow network. A closer look into the arrangement of tubes in the plasmodial network reveals a structure akin to river delta - a dense array of small tubes at the very tip of the migration front joining into an increasingly sparse network of thicker tubes towards the far end of the plasmodium (Fig. 3). The density of the tubes at the front hints at their likely 
role as samplers of information about the environment during the foraging process. The gathered information is communicated by flows across the network and processed, resulting in the observed hierarchy.

The hierarchy of the network and its impact on the organism's function is not unique to $P$. polycephalum. The study of the behavioural patterns of the fruit fly Drosopbila melanogaster revealed a previously unidentified hierarchy; its movements are organized in nested subclusters ${ }^{14}$. The conviction that this phenomenon arises from the hierarchical structure of the brain was reinforced by the first-ever simultaneous recordings of neural activity and behaviour in the worm Caenorbabditis elegans ${ }^{\text {II3 }}$. The stereotyped behaviours we identified by studying the activity of network contraction modes are strongly reminiscent to the ones found in the above listed neural organisms.

The similarity between $P$. polycephalum and neural organisms does not stop at the generation of behaviour. The organization and function of the plasmodium itself reminds of neural networks. We showed that severing a connection in the tubular network of the plasmodium induces a network-wide damage response which manifests itself in characteristic oscillation dynamics of the actomyosin-lined tubes. Intriguingly, there is evidence that muscle cells, neurons and mechanosensory receptor cells all have evolved from damage response mechanisms in a distant eukaryotic predecessor. The emergency response to extracellular calcium influx induced by wounding led to the emergence of the first mechanosensitive $\mathrm{Ca}^{2+}$ channels which gave rise to amoeboid locomotion, action potentials and, ultimately, neurons ${ }^{20,79}$. The plasmodium of P. polycephalum, being an actomyosin-lined network that locomotes in amoeboid fashion, offers exceptionally interesting insights when thinking about the evolution of the nervous system. 
However, little is known about electrical activity in $P$. polycephalum. The existence of membrane potentials in the plasmodium is long established ${ }^{59}$, but little attention has been paid to membrane potentials, since they were deemed not responsible for network-wide coordination of tube contractions ${ }^{174}$. However, the oscillations of the membrane potential correlate with the contractions ${ }^{44,180}$, indicating a possible link between the two oscillators. A good candidate for this link are the mechanosensitive $\mathrm{Ca}^{2+}$ ion channels in the plasma membrane ${ }^{162}$, whose conductivity is a function of mechanical stress - here generated by actomyosin contractions. Our results, bringing forward the multitude of contractions modes, their spatial configurations and highly dynamic nature prompt a deeper look into the electrical activity of the plasmodium.

Interestingly, the voltage-gated $\mathrm{Ca}^{2+}$ ion channels in neural organisms are responsible for short-term synaptic plasticity - change in synaptic strength essential for information processing 25 , a phenomenon reminiscent of the memory encoding mechanism in $P$. polycephalum identified in this work. Synaptic plasticity depends on the synchronization of neural activity and has a direct impact on memory and learning in organisms with a nervous system ${ }^{64}$. Studying the oscillations of membrane potentials in the context of memory encoding in $P$. polycephalum could provide valuable insight into the evolution of memory-related phenomena in living organisms. The here established setup for calcium measurement and quantification allows for such expansion, potentially simultaneously providing a recording of tube contraction activity, calcium dynamics and membrane potential oscillations.

Obtaining a complete picture of non-metabolic key processes in $P$. polycephalum would allow for studying signal processing beyond threshold alteration of transduction networks, which we elaborated on earlier in this chapter. Relying on calcium as a universal signal lead 
to the emergence of intricate signalling machinery ${ }^{16}$, relying not only on absolute concentrations of $\mathrm{Ca}^{2+}$ ions, but also making use of the temporal aspect of signalling. This way, the signal of a single chemical nature is able to elicit different responses in the cell depending on the cell state, as well as the frequency, amplitude, duration and timing of the signal ${ }^{18}$. For instance, it is known that cells generally rely on amplitude modulation in signalling and gene expression, while frequency modulation is mainly used by neurons for communicating over large distances ${ }^{\mathrm{IOI}, \mathrm{I} 48}$. The unique build of the plasmodium does not allow inferring mechanisms by analogy, thus presenting an exciting puzzle of temporal signal processing.

Investigating the signalling which governs the fusion of individual plasmodia into one organism could elucidate the transition from unicellular to multicellular organisms. $P$. polycephalum is perhaps a living embodiment of this transition; within its life cycle, the diploid plasmodium can develop from the fusion of two haploid amoebal cells ${ }^{139}$, much like the embryos of higher organisms. The similarity does not stop there; once the plasmodium is formed, its nuclei divide synchronously without cell division ${ }^{136}$, just like in the syncytial embryo of the fruit fly Drosophila melanogaster ${ }^{33}$. The improved chemotactic abilities in multicellular species are proposed as a possible reason for the emergence of multicellularity ${ }^{29}$, making the chemotactic $P$. polycephalum an ideal model system for studying this phenomenon.

We started this work by expressing surprise over the abilities of a very simple organism to display behaviour comparable to organisms with a complex nervous system. However, having identified a number of shared functioning principles conserved in P. polycephalum that seem to weave the phylogenetic tree, the apparent intelligent behaviour is less surprising. The hypothesis of the dynamic cytoskeleton and excitable membrane being enough for the development of consciousness ${ }^{8}$ is particularly easy to advocate when faced with the organism 
reactive to its environment and even able of social behaviour, while being not much more than a membrane and cytoskeleton itself. Such a shift of paradigm renders the question is Physarum polycephalum sentient? - futile. 


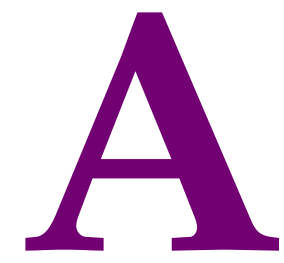

A. I Supplementary INFORMATION: ENCODING MEMORY IN TUbE DIAMETER HIERARCHY OF LIVING FLOW NETWORK 


\section{Supporting Information Text \\ Calculation of characteristic quantities}

Tube diameter. The rest tube diameter of tubes in the experiments is obtained from raw tube dynamics with a moving-average filter with a kernel width of 200 seconds to suppress rhythmic peristaltic contractions. The tube diameter is subsequently smoothed with a Gaussian filter using a kernel width of 3 minutes to account only for changes persisting longer than $\approx 2$ contraction periods. This resulting value is referred to as tube diameter both in the main text of the article and in the SI Appendix. The rest tube diameters obtained in the simulations was obtained analogously by applying a moving-average filter of a width corresponding to the oscillation period in the simulation. The smoothing step was skipped due to absence of noise.

Relative tube growth. The relative tube growth in Fig.2-B for each tube is calculated as the ratio of mean tube diameter 5 min min before the end of the experiment and mean tube diameter 5 min before stimulus application.

Relative tube diameter. The relative tube diameter in Fig. 3 for each tube is calculated as the ratio of tube diameter and mean tube diameter before stimulus application.

Automatic classification of characteristic tube dynamics. The automatic classification of characteristic tube dynamics upon stimulus application resulting in green and blue domain in Fig. $4 \mathrm{C}$ was carried out by mapping the peaks of the diameter dynamics before and after the stimulus. Tubes whose diameter increased rapidly and significantly after the application of the stimulus are those marked in blue. Tubes that upon stimulus application first undergo a significant decrease in diameter, followed by an increase are marked in green. Tubes whose dynamics doesn't belong to either category, such as steadily shrinking tubes, are marked in gray. The dynamics depicted in Fig. $4 \mathrm{C}$ correspond to the mean diameters of the two characteristic ensembles.

Instantaneous growth. The instantaneous tube growth in Fig. 5 A was calculated as the numerical time derivative of tube diameter.

i)

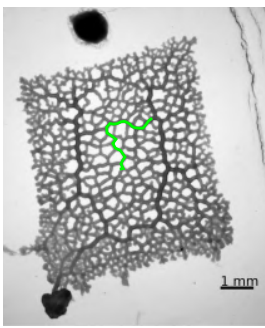

iii)

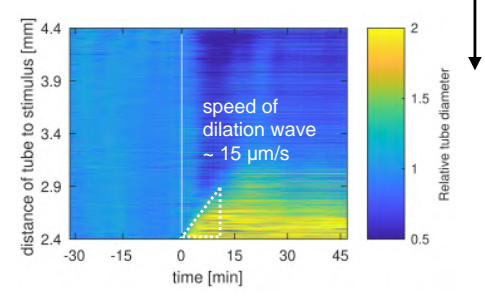

ii)

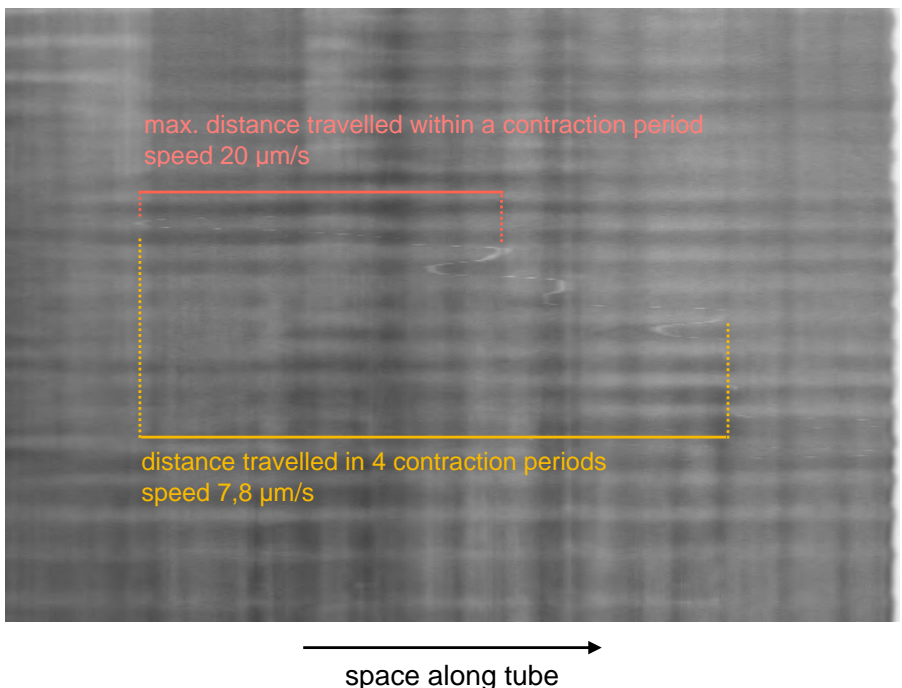

Fig. S1. Measurement of flow-based transport speed. i) Trajectory of a tracked particle marked in green. ii) Kymograph along the trajectory marked in green. Trace of a larger particle transported by flow allows for flow speed measurement. Within a contraction period, the particle reached a speed of $20 \mu \mathrm{ms}{ }^{-1}$, while the average speed over 4 contraction period amounts to $7.8 \mu \mathrm{ms}^{-1}$. iii) Speed of the post-stimulus dilation wave amounts to $15 \mu \mathrm{ms}^{-1}$, confirming the hypothesis of flow-transported signal. 

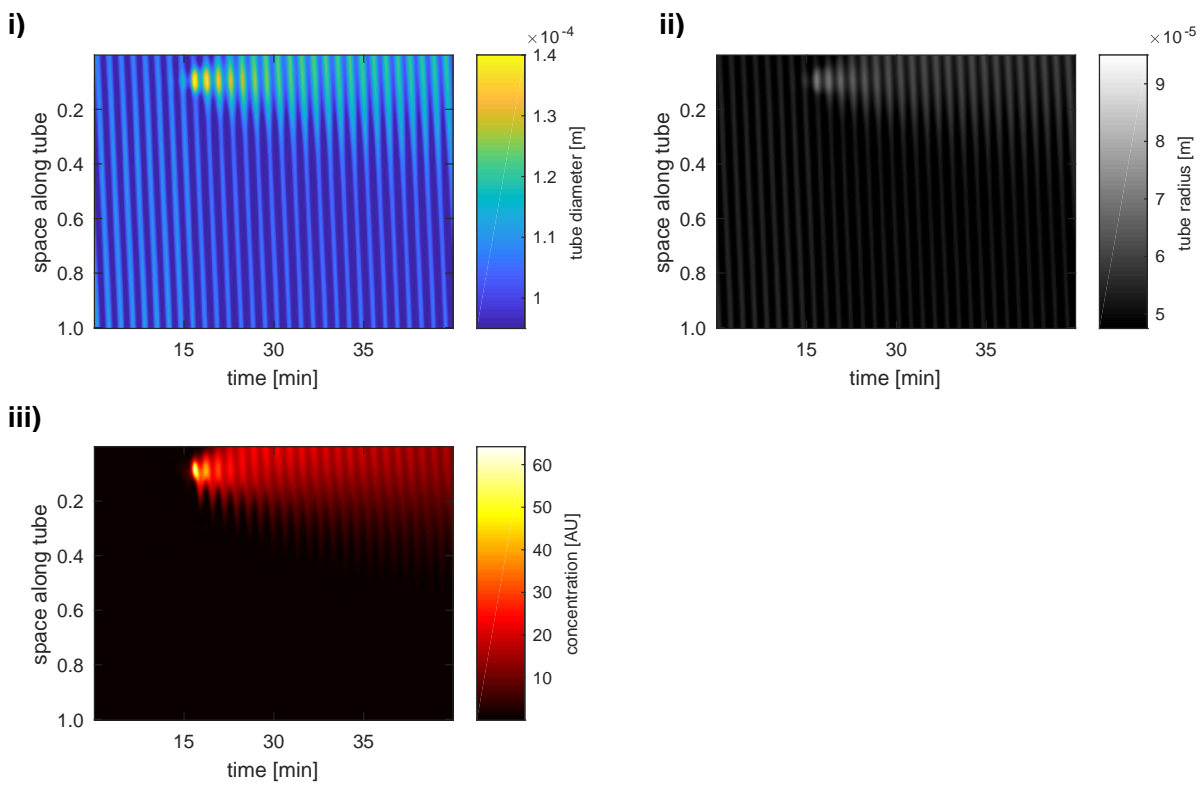

Fig. S2. (i) Kymograph of tube diameter along the full length of the simulated tube. The release of the chemical agent starts at about . The chemical agent causes a decrease in tube elasticity, resulting in tube diameter increase along the tube as it spreads by fluid flow. (i) Kymograph of the tube radius. iii) Kymograph depicting the spread of the chemical agent along the tube.

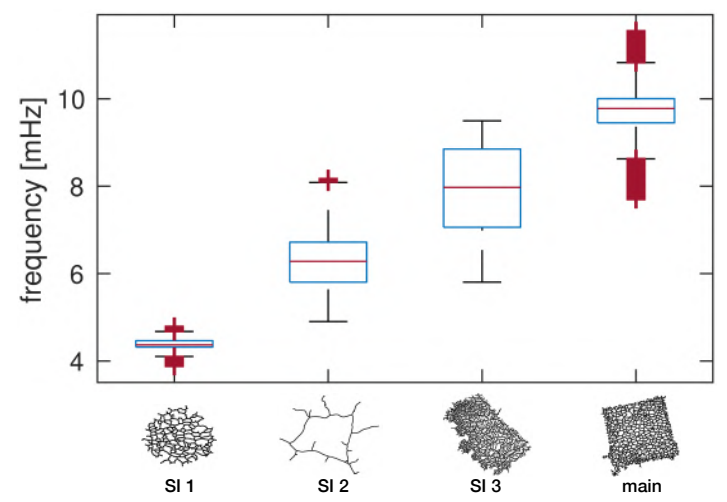

Fig. S3. Contraction frequencies of the plasmodium tubes of all analyzed data sets before stimulus application. Central red mark denotes the median, top and bottom box edges 75 th and 25 th percentile, whiskers enclose the range of data points and red plus symbols indicate the outliers.

\section{Supplemental data sets}

In the following figures, results of the data analysis applied to the supplemental data sets are united in a single figure for each data set for more clarity. In every figure, the data is analysed and presented identically to the one of the main text: i) corresponds to Fig.2, ii) to Fig.3, iii) to Fig.4-C and iv) to Fig.5-A, and v) to Fig.5-C.

In supplemental data sets 1 and 3, multiple nutrient stimuli were given to demonstrate that the observed network response is robust and reproducible. In supplemental data set 1 , two stimuli were given spaced in time at opposing sides of the network. In supplemental data set 3, first two stimuli were given on opposing sides of the network's short axis. After relaxation time of $48 \mathrm{~min}$, a following stimulus was given at the long end of the network. The measurements presented in the following figures show a reproducible response of the network to nutrient stimuli across different network sizes and morphologies, as well as nutrient sources and their placement. 
i)

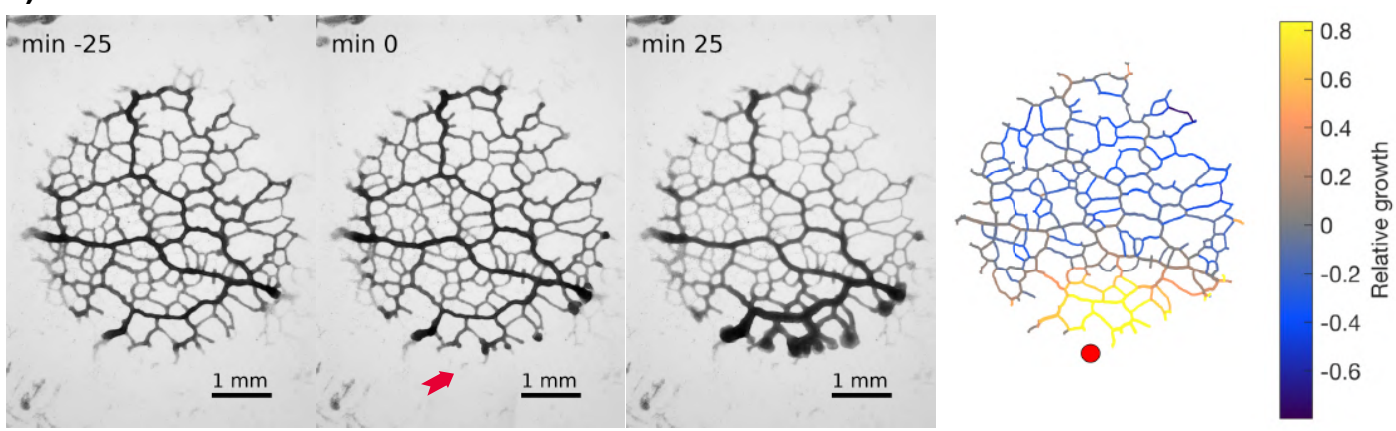

ii)

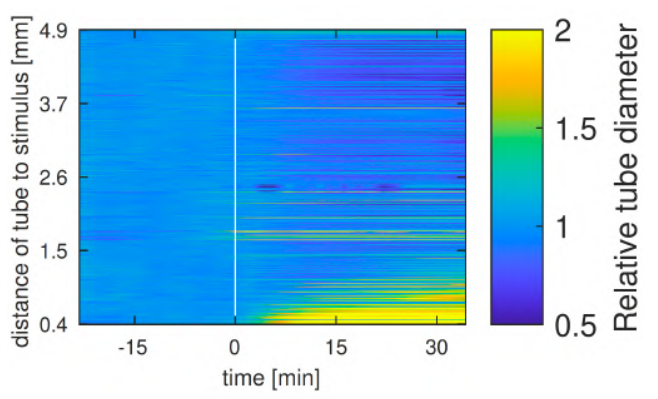

iii)
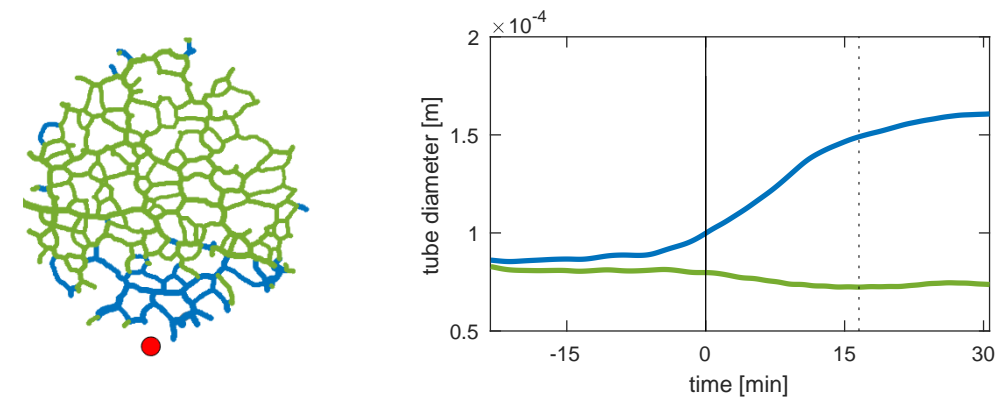

iv)
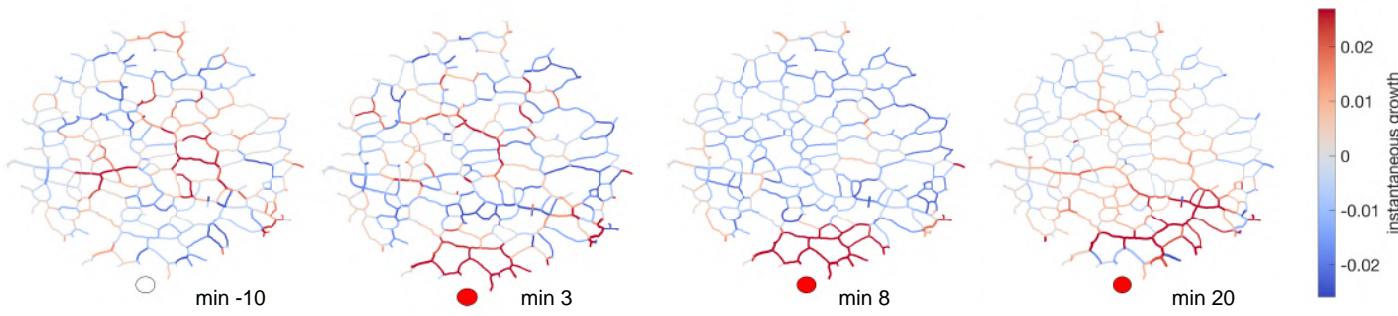

v)
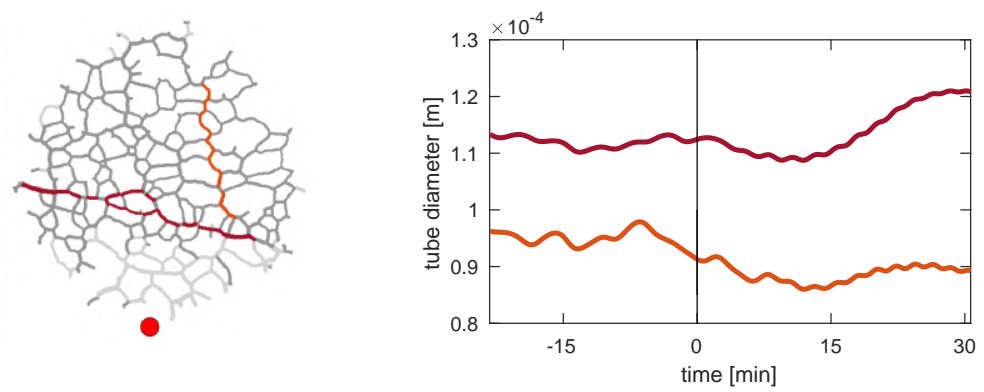

Fig. S4. Supplemental data set 1 , application of the first stimulus. 
i)
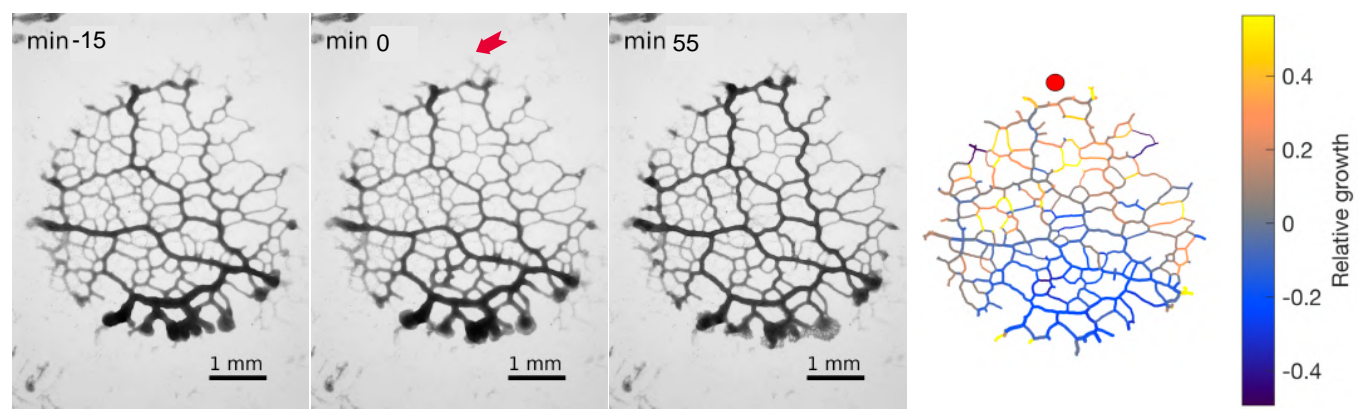

ii)

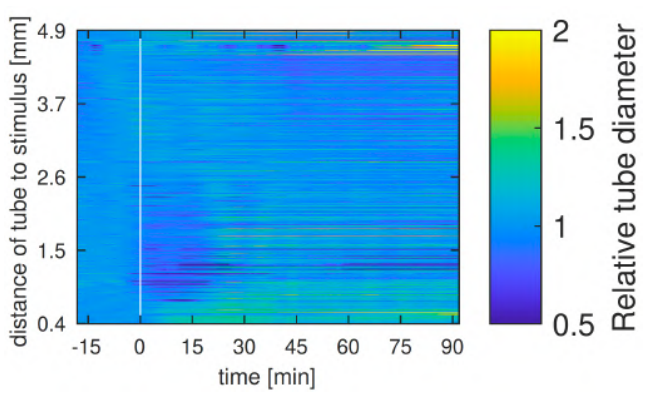

iii)
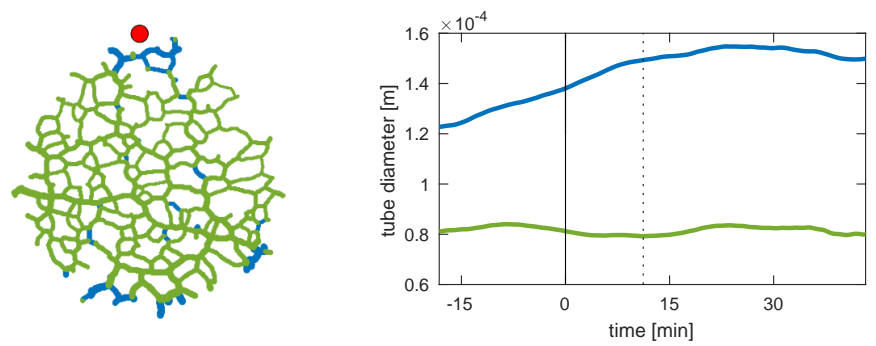

iv)
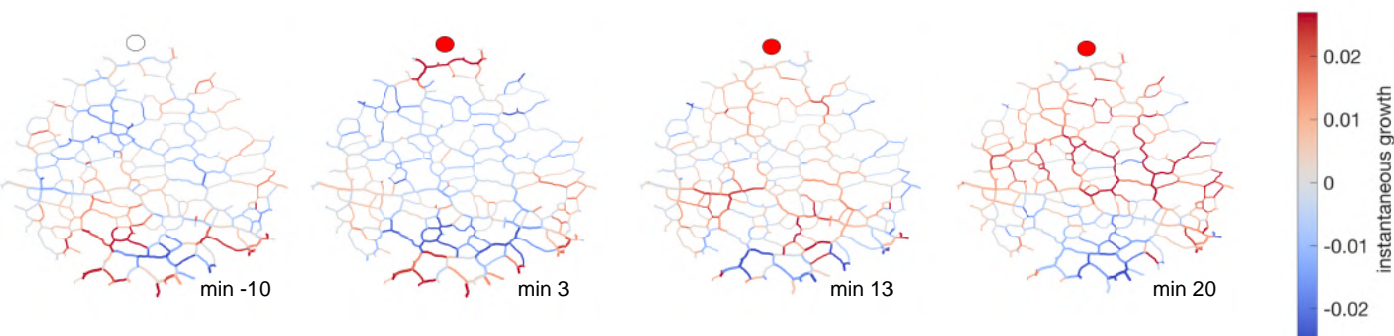

v)
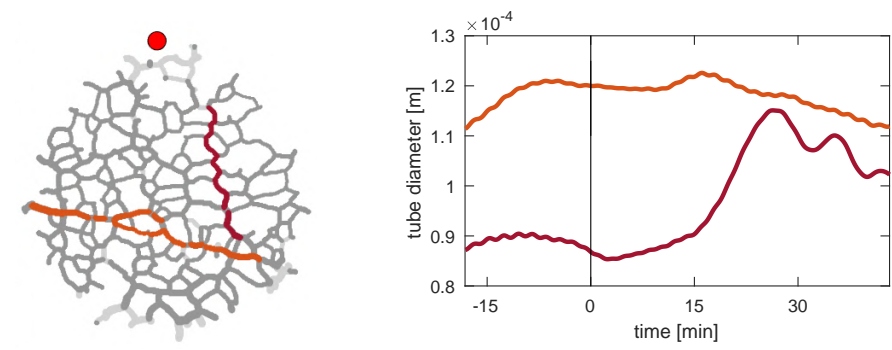

Fig. S5. Supplemental data set 1, application of the second stimulus. 
i)

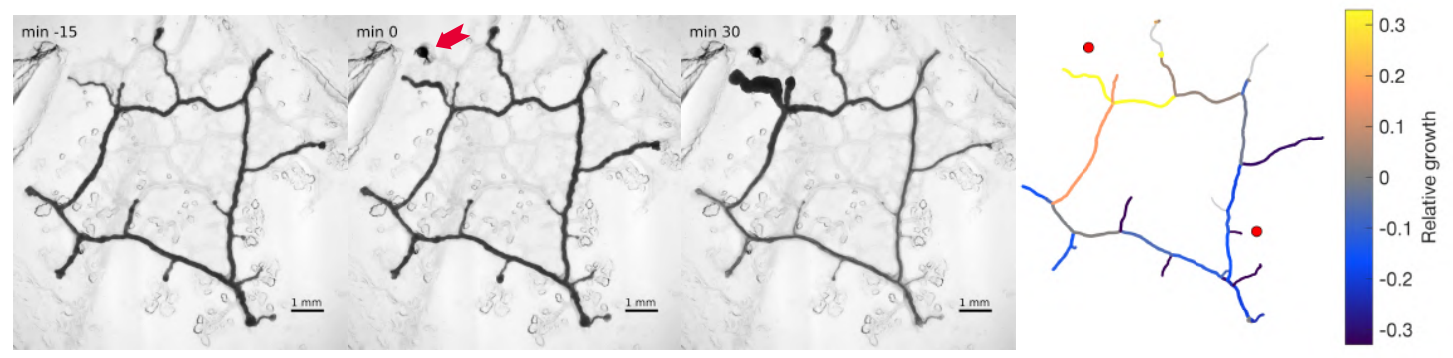

ii)

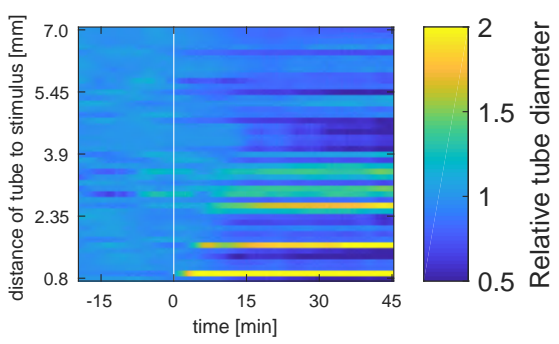

iii)
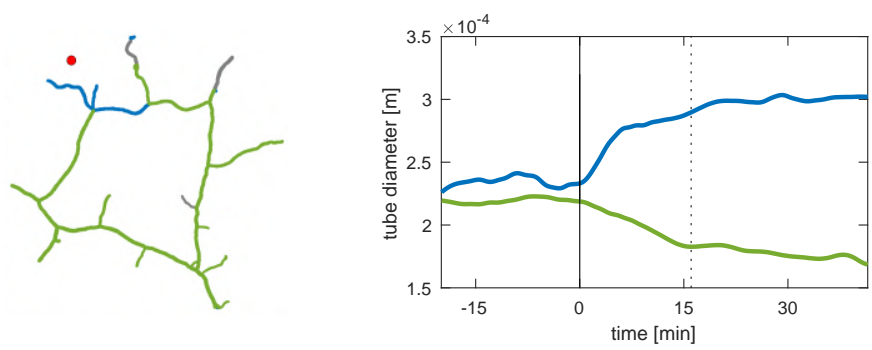

iv)
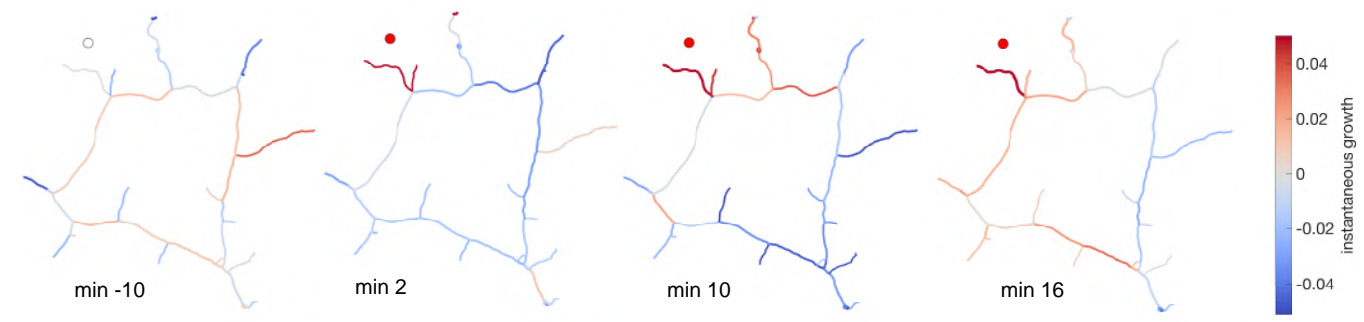

v)
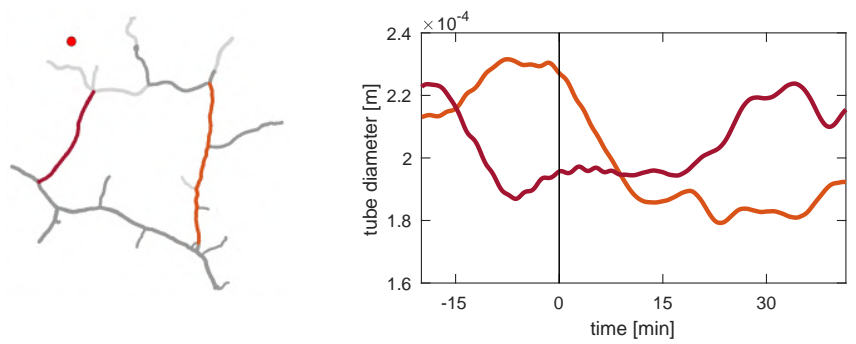

Fig. S6. Supplemental data set 2 . 
i)
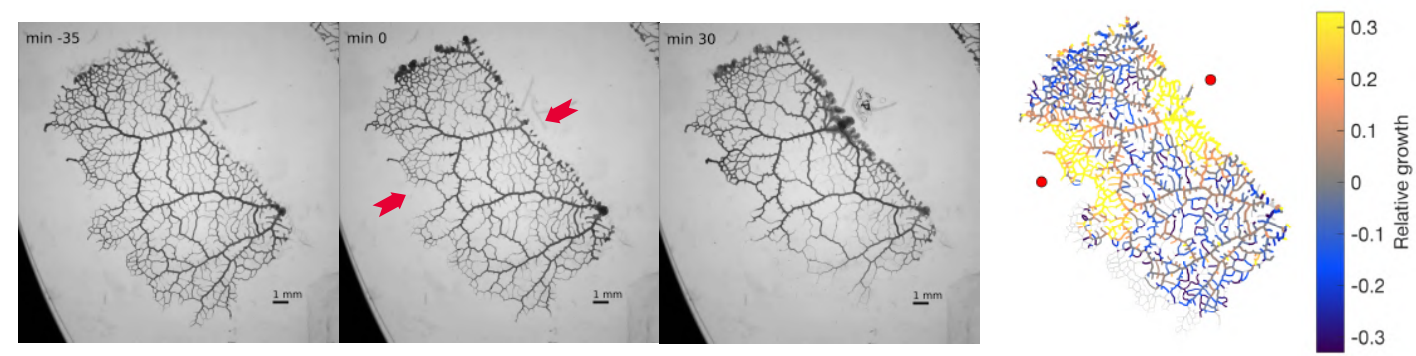

ii)

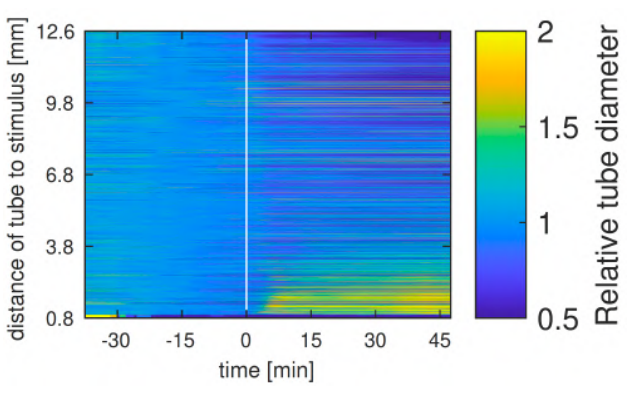

iii)
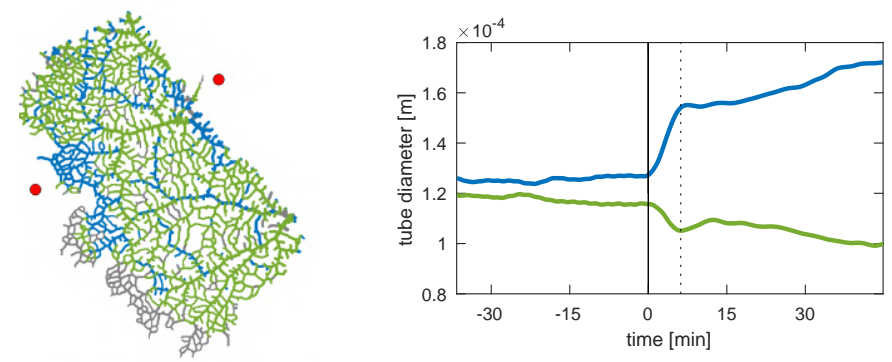

iv)
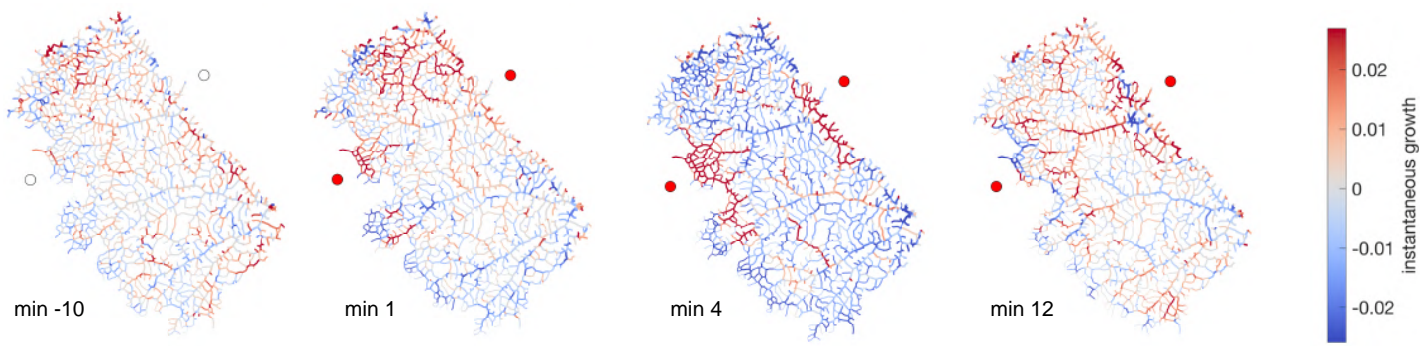

v)
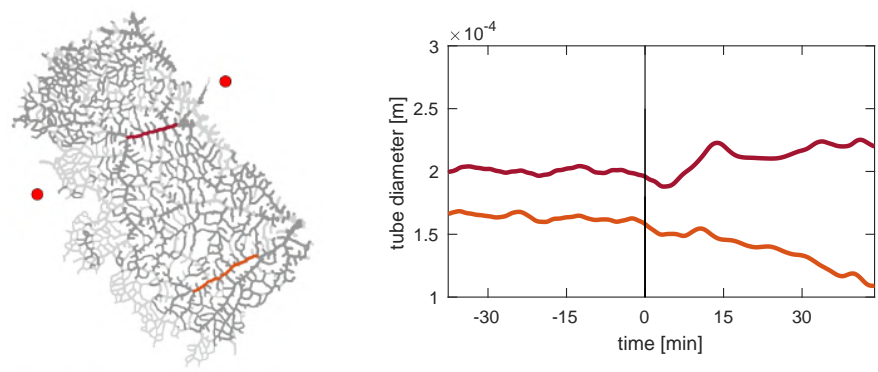

Fig. S7. Supplemental data set 3 , simultaneous application of the first two stimuli. 
i)
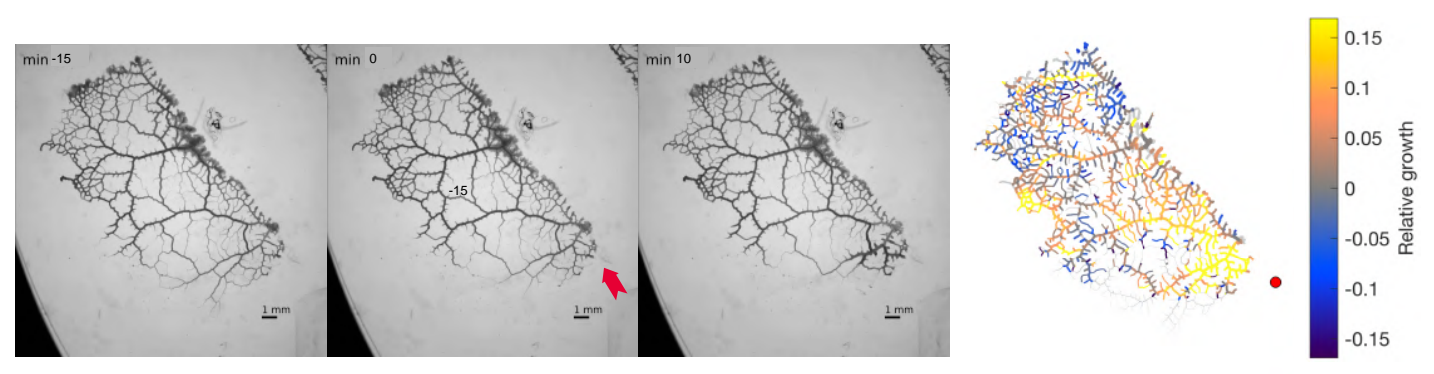

ii)

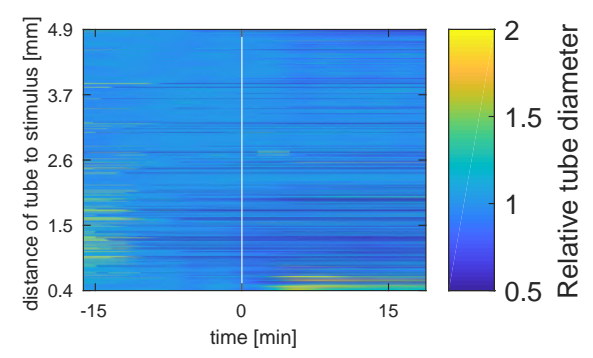

iii)
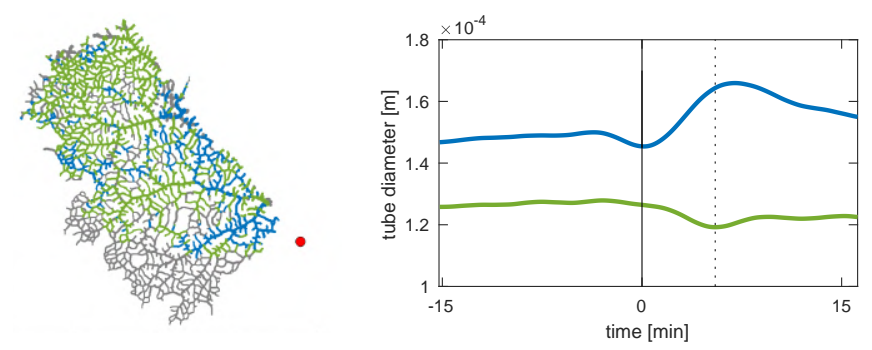

iv)
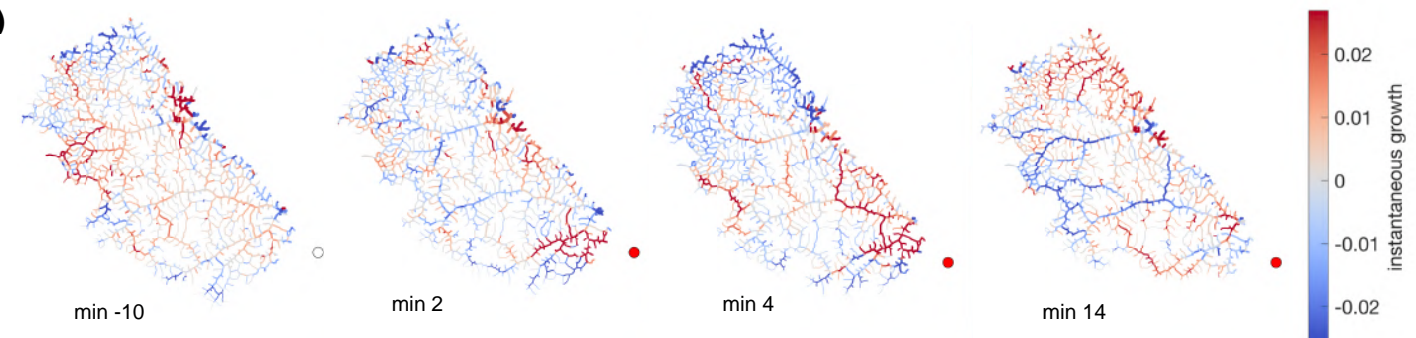

v)
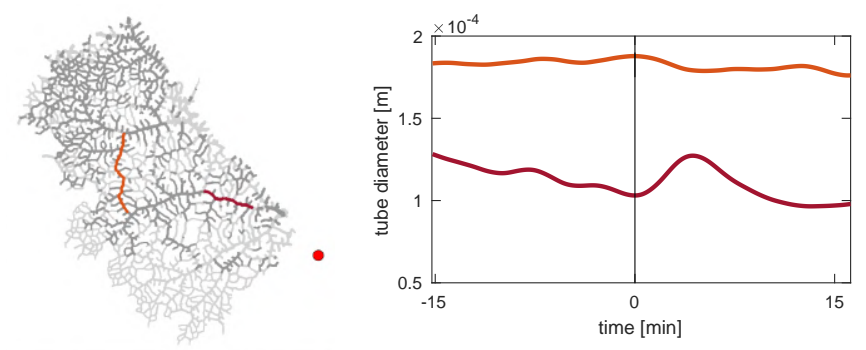

Fig. S8. Supplemental data set 3 , application of the third stimulus. 


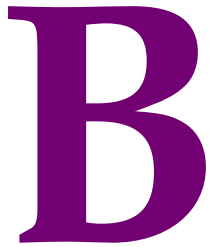

B. I SUPPLEMENTARY INFORMATION: EMERGENCE OF BEHAVIOR IN A SELF-ORGANIZED LIVING MATTER NETWORK

IOO 
Data processing. Our data is a stack of bright-field images recorded from the $P$. polycephalum network with a rate of one frame every three seconds. Each bright-field frame has a time label $t_{i}$ and the total number of frames is given by $T$. We process this data in the following steps. First, we mask the network in the bright-field images through thresholding. It is important to note that we use the same mask for all the images in the stack. This is possible since we consider a network that does not significantly move or change its morphology. This is even true when we apply a stimulus to the network, since we only consider the initial stages of stimulus response, before the network starts to display strong movement. From the masked regions of the bright-field frames we extract pixel intensity values which we convert to 8-bit format. Since we are here primarily interested in the contraction dynamics of the organism and not in the the actual base thickness of tubes or its long-term growth dynamics, we detrend the data. This leaves us only with the desired information about contractions taking place in the time scale of several minutes. We store the intensity values of each frame in a vector $\vec{I}^{t_{i}}$ of dimension $M$ equal to the number of pixels in the network and $i$ indexes the frames has the range $i=1, \ldots, T$. From the post-processed data we define the following data matrix

$$
\mathbf{X}^{t}=\left(\vec{I}^{t_{1}}, \vec{I}^{t_{2}}, \ldots, \vec{I}^{t_{T}}\right)
$$

where $t$ denotes the matrix transpose.

Principal Component Analysis (PCA). The contraction modes are computed from the covariance matrix of the data. We compute the covariance matrix from the data matrix $\mathbf{X}$ after centering each column. The covariance matrix is given

$$
\mathbf{C}=\frac{1}{T-1} \mathbf{X}^{t} \mathbf{X}
$$

The sought after contraction modes $\vec{\phi}_{\mu}$ are the eigenvectors of the covariance matrix

$$
\mathbf{C} \vec{\phi}_{\mu}=\lambda_{\mu} \vec{\phi}_{\mu}
$$

and $\lambda_{\mu}$ is the eigenvalue. The number of non-zero eigenvalues is equal to the rank of the covariance matrix. The eigenvalue captures the variance of the data along the direction of mode $\vec{\phi}_{\mu}$. We also define the relative eigenvalue as

$$
\tilde{\lambda}_{\mu}=\frac{\lambda_{\mu}}{\sum_{\nu=1}^{T} \lambda_{\nu}} .
$$

The mode coefficient $a_{\mu}$ is obtained by projecting the data onto mode $\vec{\phi}_{\mu}$.

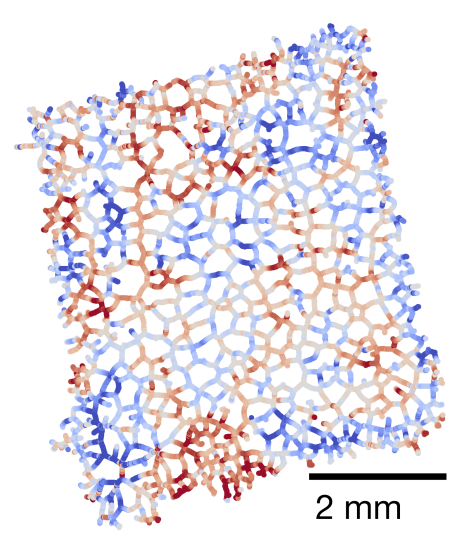

Fig. S1. Mode $\vec{\phi}_{30}$. 


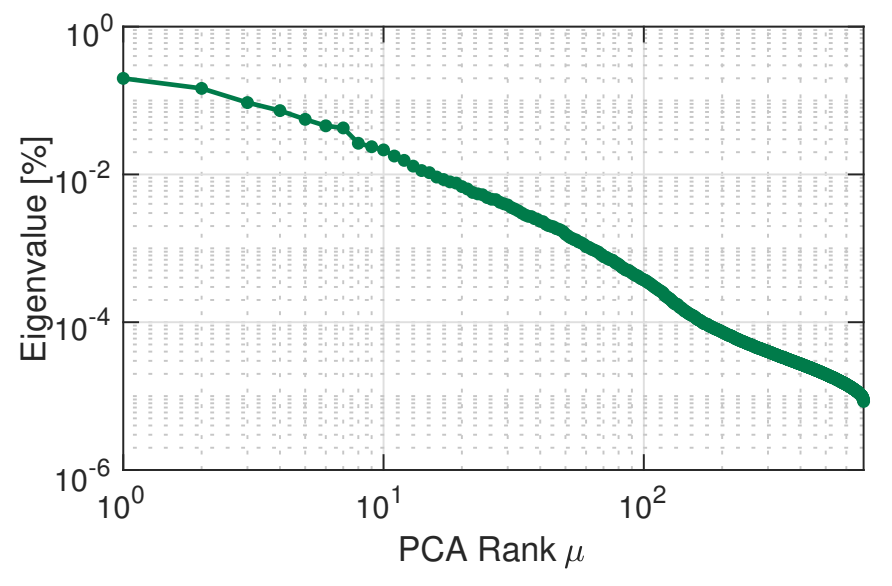

Fig. S2. Eigenvalues of the large rectangular with a stimulus over 700 frames.

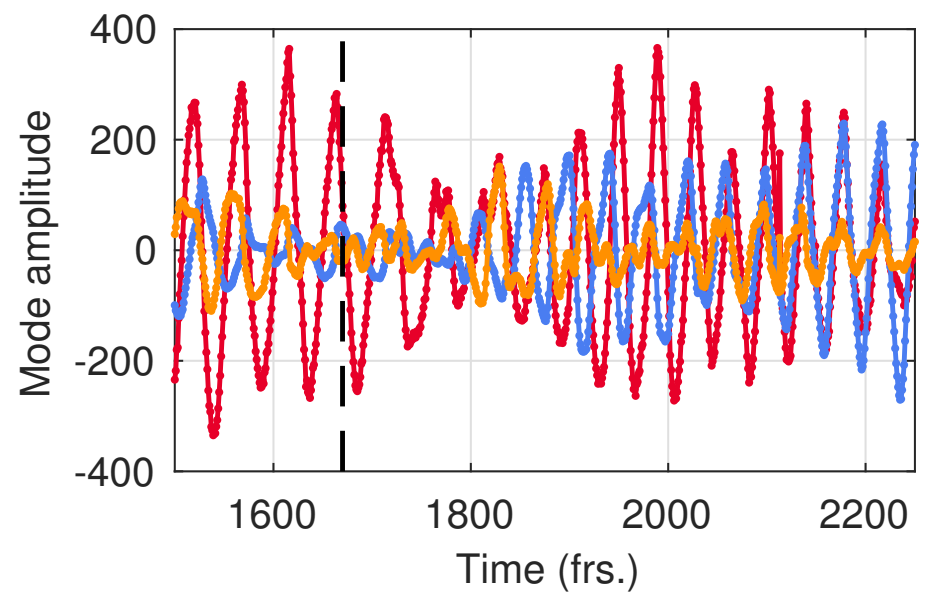

Fig. S3. Coefficients of the three top-ranked modes $\vec{\phi}_{1}, \vec{\phi}_{2}$ and $\vec{\phi}_{3}$ shortly before and after application of the stimulus to the large rectangular network.

Flow rate calculation in a cell with single tube morphology. To compute the flow rate of the cytoplasm in a $P$. polycephalum specimen with single tube morphology we use the theory developed in (1). In this work an incompressible Newtonian fluid in a tube of fixed length and given time-dependent thickness profile is considered and the equations for the flow velocity field are written in the lubrication theory approximation. Assuming no-slip boundary conditions, the field is fully determined at every point along the tube in terms of the time-dependent thickness profile. For the case when the tube profile is a periodic train of waves, we can calculate the volume flow rate averaged over a period. This serves to characterise the performance in pumping of the significantly contracting $P$. polycephalum cell. We determine the time period over which to average the volume flow rate directly from the flow rate curve. In our computations we assume a value of the viscosity equal to one.

Mode superpositions in a cell with single tube morphology. We are interested in how the contraction dynamics of the cell controls the cell's locomotion behaviour. In our analysis we therefore focus on the tube segment connecting the locomotion fronts at either end of the tube and perform Principal Component Analysis only on this part of the cell. Since the tube is effectively one-dimensional, we find that the modes that we obtain closely approximate Fourier modes. This means that superpositions of these modes afford a clear interpretation in terms of different contraction wave patterns. Such an interpretation is even further facilitated by the fact that we find that over large time intervals after the stimulus, the number of significant modes is very small. Indeed, over such time intervals it is sufficient to approximate the contraction dynamics with only one or two modes, as can be seen from Figs. S4 and S5. Hence we are essentially studying a superposition of modes $\vec{\phi}_{1}$ and $\vec{\phi}_{2}$ shown in Fig. 5A of the main text with their oscillating mode coefficients shown in Fig. S6. To develop a more intuitive understanding of the superposition, we first note that the modes $\vec{\phi}_{1}$ and $\vec{\phi}_{2}$ approximate sine and cosine functions over the length of the tube. Further we see that the coefficients in Fig. S6 change in amplitude and phase relative to each other. As a result the superposition of these two modes changes in its nature over time, ranging form a purely standing wave to a purely left-traveling wave. 


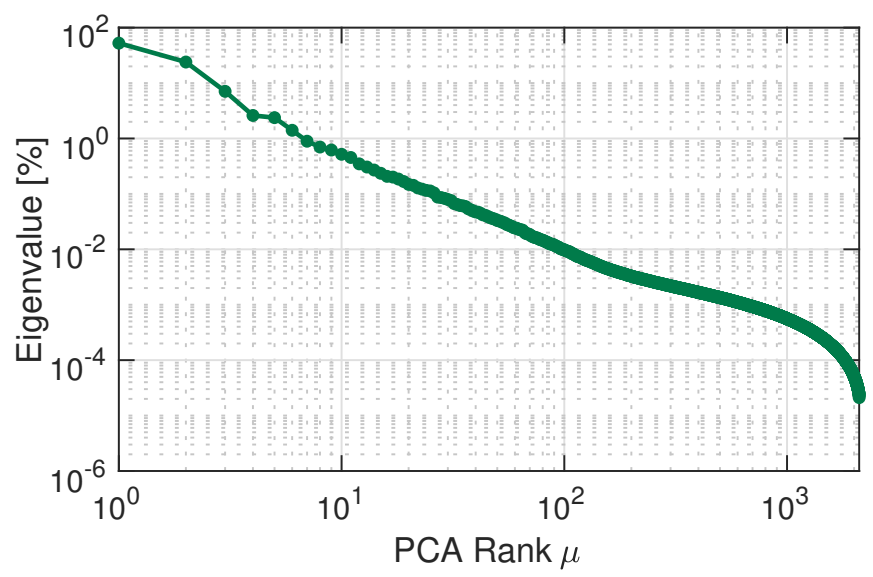

Fig. S4. Spectrum of relative eigenvalues for the single tube.

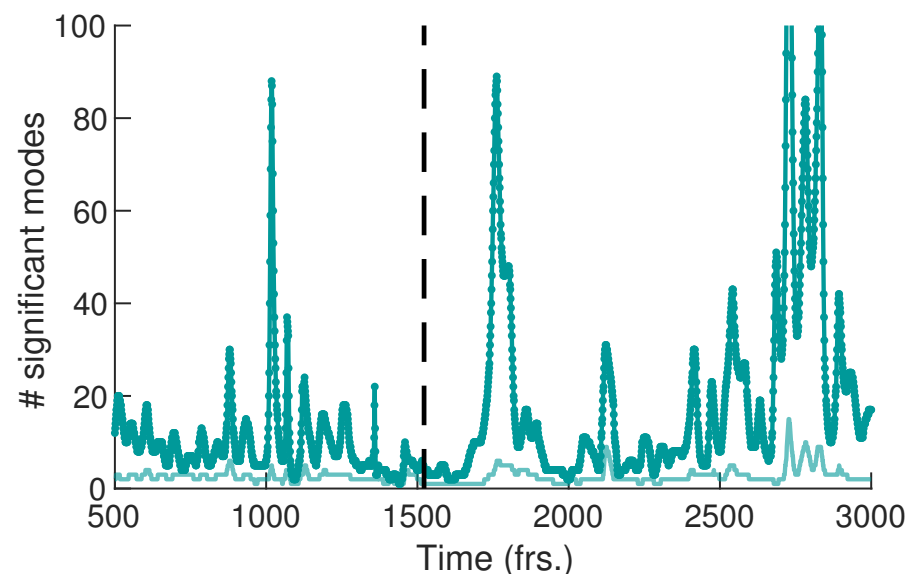

Fig. S5. Number of significant modes for the single tube over time for $70 \%$ (light green) and $90 \%$ (dark green) cutoff. The time of stimulus application is indicated by the dashed line.

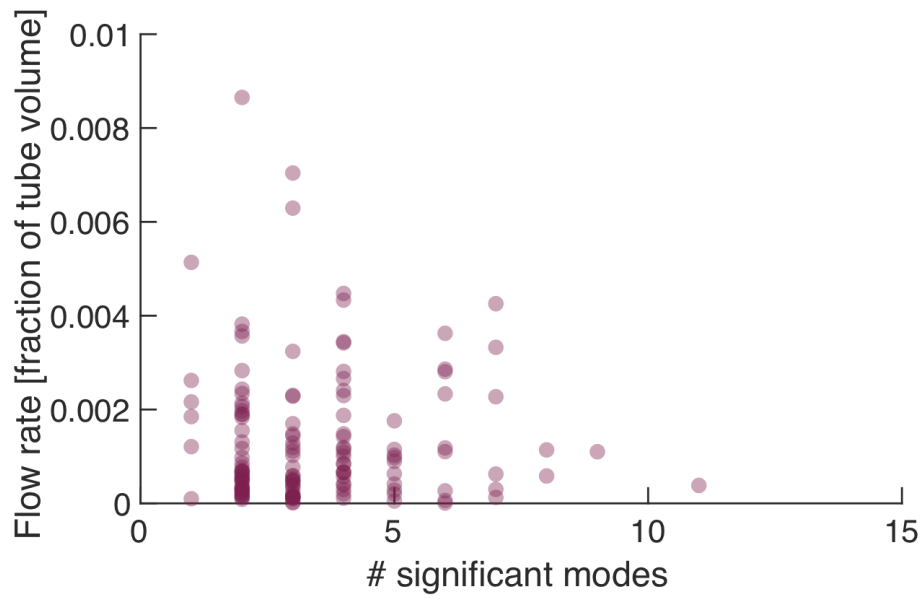

Fig. S6. Volume flow rate at the right end of the tube. 


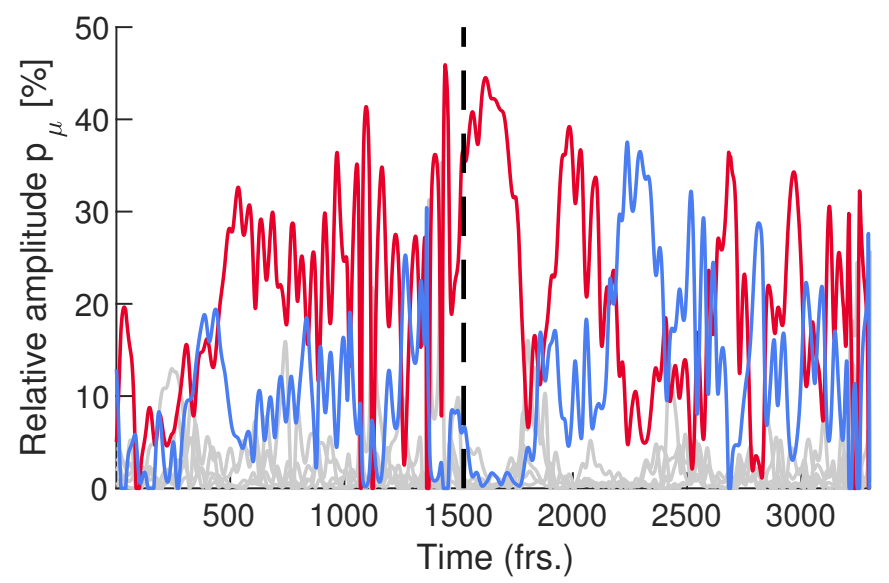

Fig. S7. Relative amplitudes of the seven top-ranked modes of the single tube. Relative amplitude of mode $\vec{\phi}_{1}$ (red), mode $\vec{\phi}_{2}$ (blue) and modes $\vec{\phi}_{3}$ to $\vec{\phi}_{7}$ in gray.

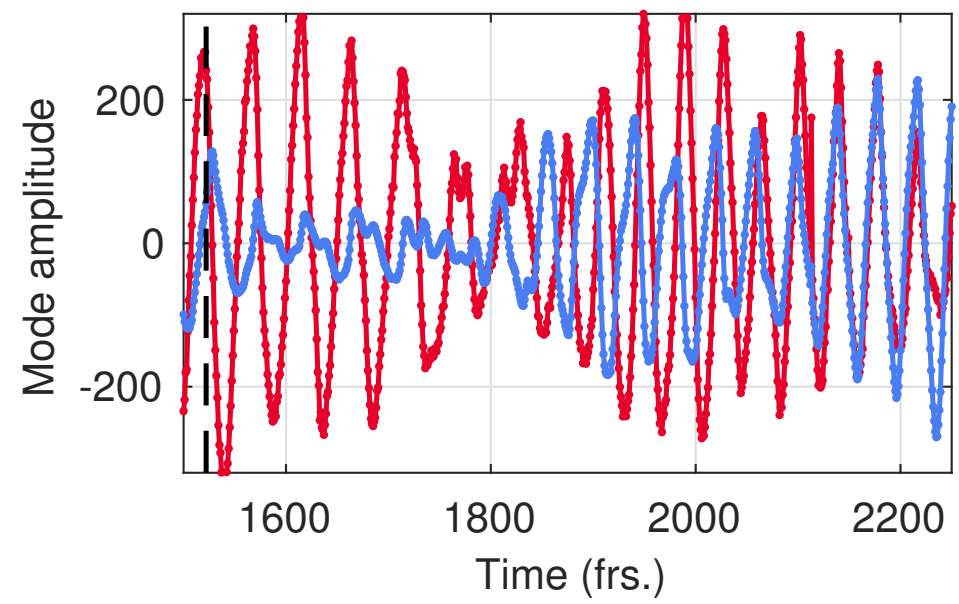

Fig. S8. Mode coefficients of first (red) and second mode (blue) after the application of the stimulus (dashed line) to the single tube.

41 References

42 1. M Li, J Brasseur, Non-steady transport in finite-length tubes. J. Fluid Mech. 248, 129 - 151 (1993). 


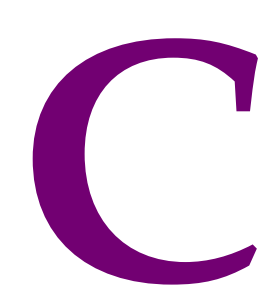

C.i Supplementary information: Spatial mapping Reveals multi-step patTERn Of WOUND HeALING IN PHYSARUM POLYCEPHALUM 


\title{
Supplemental Information: Spatial mapping reveals multi-step pattern of wound healing in Physarum polycephalum
}

\author{
Felix Bäuerle*, Mirna Kramar*, Karen Alim \\ Max Planck Institute for Dynamics and Self-Organization, D-37077 Göttingen, \\ Germany \\ * Authors contributed equally to this work. \\ E-mail: karen.alim@ds.mpg.de
}

\section{S1. Supplemental Text: Preparation of microplasmodia and buffer recipes}

The microplasmodia for the shaking culture are grown from a healthy network feeding on heat-killed HB101 bacteria. A section of network is transferred together with the agar slice into a new plate without food. Once the network crawls onto the agar in the new plate, the agar around the network is excised from the dish and the dish is filled with the a 1:1 mixture of SDM and BSS medium up to the agar level, resulting in an agar island with the network on it.

The dish is left to incubate for several (2-4) days until the plasmodium starts to form a network in the liquid medium. The floating network is gently transferred to a flask containing $15 \mathrm{ml}$ of SDM and $25 \mathrm{ml}$ of BSS and kept in a shaking culture (150 rpm), where it breaks into microplasmodia.

The microplasmodia need to be resuspended every 2-3 days. $3 \mathrm{ml}$ of microplasmodial culture is aspirated with a pipette, transferred to a new flask with $25 \mathrm{ml} \mathrm{SDM}$ and $25 \mathrm{ml}$ BSS, and stored in the shaker.

\section{S1.1. Preparation of the SDM buffer}

For $1 \mathrm{l}$ of SDM buffer, dissolve $10 \mathrm{~g}$ of glucose, $10 \mathrm{~g}$ peptone from soybean, $3.54 \mathrm{~g}$ citric acid monohydrate, $2 \mathrm{~g} \mathrm{KK_{2 }} \mathrm{PO}_{4}, 1.026 \mathrm{~g} \mathrm{CaCl}_{2} \times 2 \mathrm{H}_{2} \mathrm{O}, 0.6 \mathrm{~g} \mathrm{MgSO}_{4} \times 7 \mathrm{H}_{2} \mathrm{O}, 0.0034 \mathrm{~g}$ $\mathrm{ZnSO}_{4} \times 7 \mathrm{H}_{2} \mathrm{O}, 0.0042 \mathrm{~g}$ thiamine- $\mathrm{HCl}$, and $0.0016 \mathrm{~g}$ biotin in $0.8 \mathrm{l}$ of water. Adjust the $\mathrm{pH}$ to 4.6 with $\mathrm{KOH}$, fill the rest of the water and autoclave for $2020 \mathrm{~min}$. Store at room temperature. Before use, add $10 \mathrm{ml}$ of hemin solution.

\section{S1.2. Preparation of the BSS buffer}

For $1 \mathrm{l}$ of BSS buffer, dissolve $3 \mathrm{~g}$ of citric acid monohydrate, $4.20 \mathrm{~g} \mathrm{~K}_{2} \mathrm{HPO}_{4} \times 3 \mathrm{H}_{2} \mathrm{O}$, $0.25 \mathrm{~g} \mathrm{NaCl}, 0.21 \mathrm{~g} \mathrm{MgSO}_{4} \times 7 \mathrm{H}_{2} \mathrm{O}, 0.05 \mathrm{~g} \mathrm{CaCl}_{2} \times 2 \mathrm{H}_{2} \mathrm{O}$ in $0.8 \mathrm{l}$ of water. Adjust the $\mathrm{pH}$ 
to 5.0 with $\mathrm{KOH}$, fill the rest of the water and autoclave for $20 \mathrm{~min}$. Store at room temperature.

\section{S2. Supplemental Text: Hilbert transformation}

This supplemental information is based on [1]. To obtain the quantitative key parameters namely amplitude $A$, frequency $f$ and phase $\varphi$ of contractions, we use the Hilbert transformation.

The Hilbert transform $h(t)$ of a function $a(t)$ is defined as:

$$
h(t)=\frac{1}{\pi} \int_{\infty}^{\infty} \mathrm{d} \tau \frac{a(\tau)}{t-\tau}
$$

where $h(t)$ is phase shifted by $-90^{\circ}$ for all positive frequencies and by $90^{\circ}$ for all negative frequencies. With the real function $a(t)$ and its Hilbert transform $h(t)$ one can form the complex analytical signal

$$
c(t)=a(t)+i h(t)
$$

which can be used to derive the instantaneous amplitude

$$
A(t)=|c(t)|
$$

and phase

$$
\varphi(t)=\arg (c(t))
$$

The frequency is given by differentiating the phase $f=\dot{\varphi}$.

For the presented results each data point (skeleton pixel) is treated as a discrete time series which can be Hilbert transformed accordingly into an analytical signal. To approximate the analytic signal from discrete data, we calculate the fast-fourier transform (FFT) of the input sequence, replace those FFT coefficients that correspond to negative frequencies with zeros, and calculate the inverse FFT of the result [2].

The obtained key parameters are stored as a set for each pixel, i.e. $[a(t), A(t), \varphi(t), f(t)]_{k}$ where $k$ represents all pixels in space.

\section{S3. Supplemental Text: Kymograph based analysis}

As described in section 2.1 the control of plasmodia network parameters like fan amount, motility, size or grid density is limited mostly to selection of parallel culturing. Therefore quantitative analysis as described in 2.2 is not suitable for every experiment. To analyse the bulk of experiments we use a kymograph based approach described in the following. A kymograph is a 3D representation of designated data with one space coordinate, one time coordinate and one value (intensity) coordinate. In our case space is represented as line through a main vein in a network (compare with E1, red line). Along this line a 3 px orthogonal average of the light intensity is taken for the value coordinate. This is done for every image and concatenated in time.

Along the time coordinate the Hilbert transform is performed for every point in space 
(see S2). Here, additionally, the amplitude is computed with a root-mean-square moving average (RMS-MA) filter besides the absolute value of the analytical signal. After computation the results are averaged in space and plotted in a line plot to depict contraction dynamics.

\section{Bibliography}

[1] Nobach H 2007 Gegen Wavelets \& Co. (Shaker Verlag) ISBN 978-3-8322-6564-9

[2] Marple L 1999 IEEE Transactions on signal processing 47 2600-2603

\section{S4. Supplemental Figures}
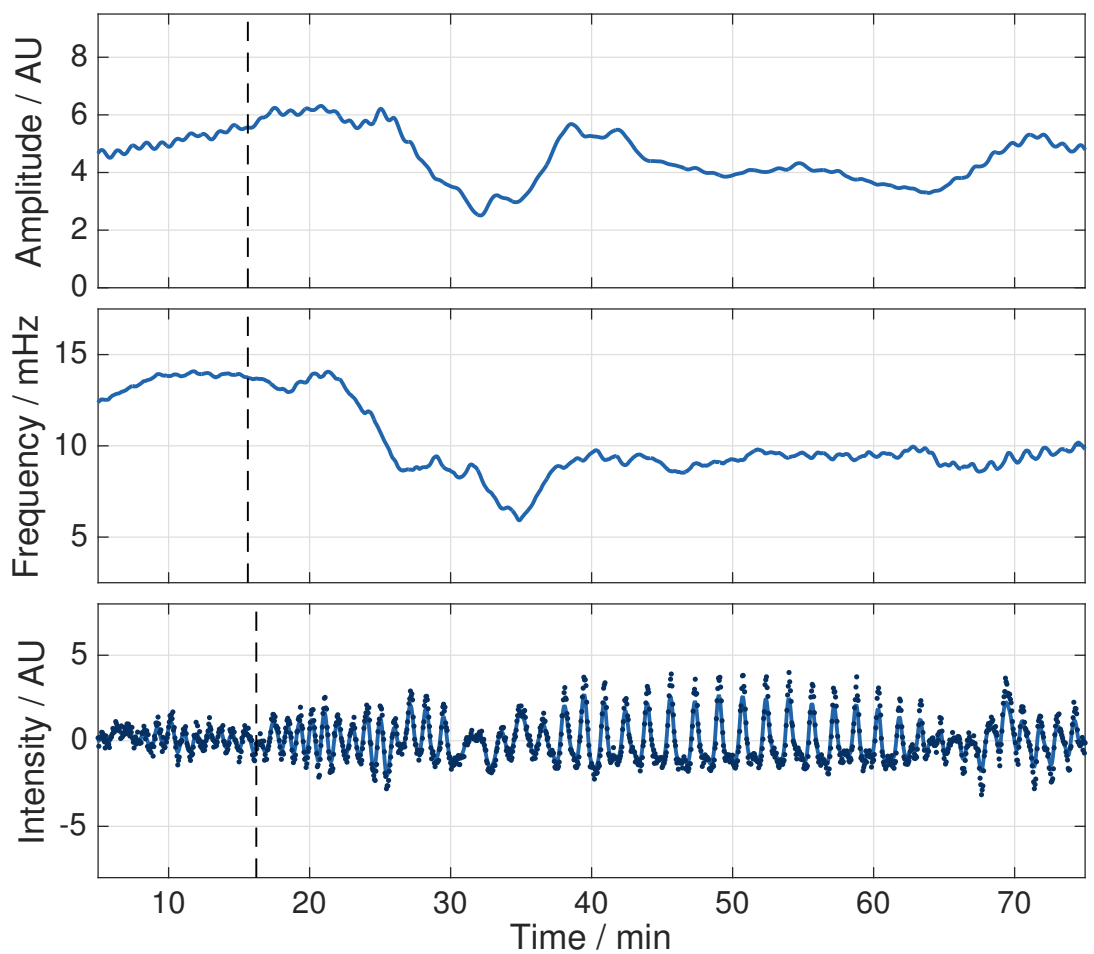

Figure S1. Analysis of averaged data from the whole network presented in the main text. The dotted line indicates the cutting event. Dotted points in the intensity plot show raw data and the line depicts Gaussian filtered data. Dynamics of small subnetwork are lost in comparison to Fig. 5. 


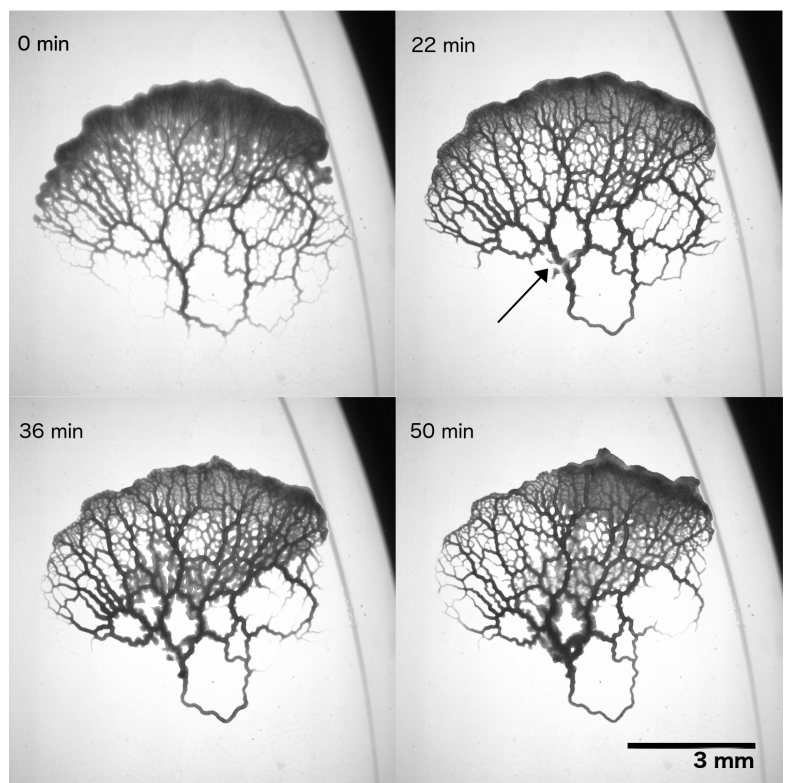

Figure S2. Material is transported towards the cut site (arrow). In the minutes after the cut, body mass accumulates along the general direction of the cut site and fans develop around it. 


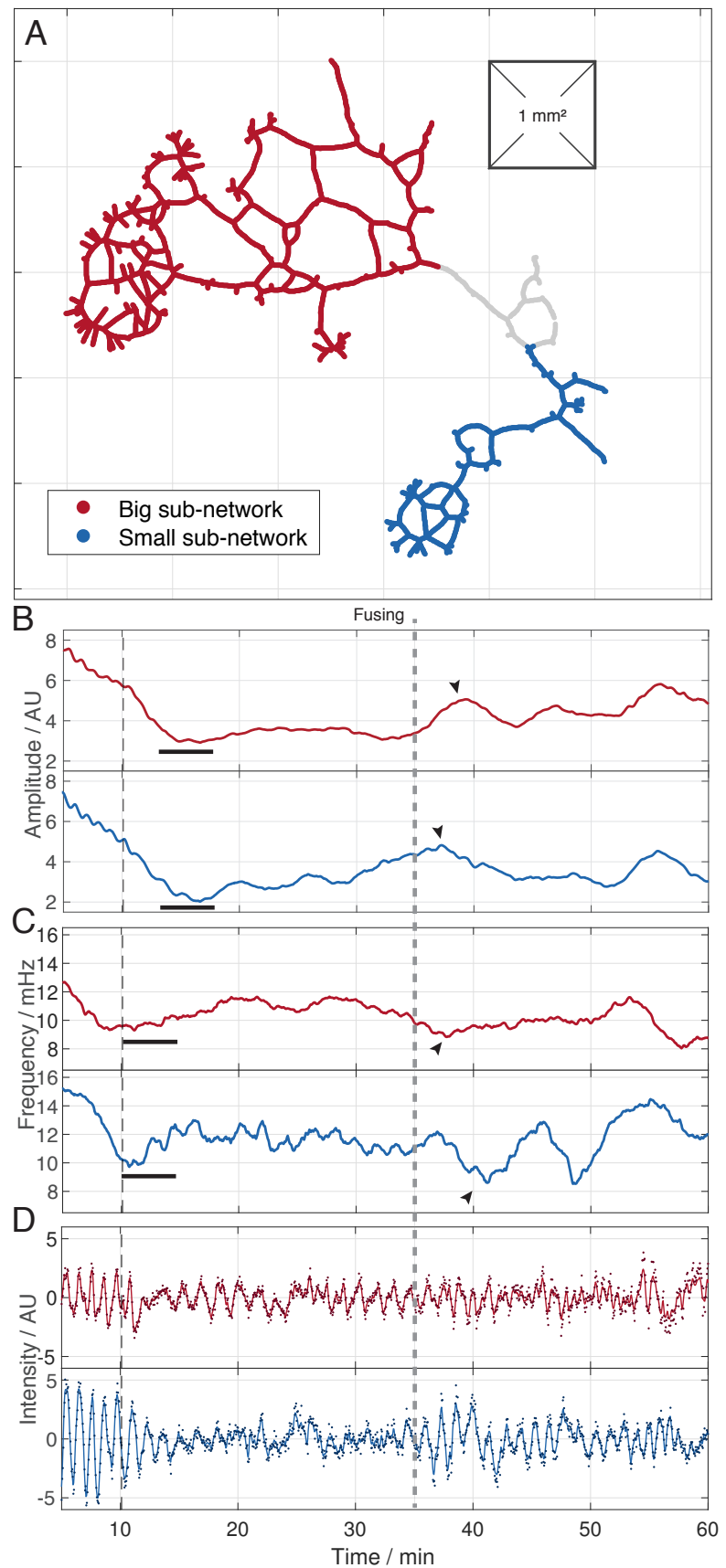

Figure S3. Full quantitative analysis of a second dataset (E3) for comparison. Oscillation parameters in the big- and small-sub-network depicted in (A) which result from the cut. Grey area (cut site) is not considered in the analysis. Time series of amplitude (B), frequency (C) and intensity (D) are averaged in the respective domains and compared; top : big sub-network, bottom: small sub-network. In each of these plots the black dashed line indicates the moment of the cut. The grey dashed line marks the time point of fusion. Black bars underline periods of stalling in (B) and (C). In (D) the red and blue solid lines represents the Gaussian filtered intensity (kernel width $=39 \mathrm{~s}$ ) and markers show raw averaged data. Black arrows indicate respective extremal peaks in the transition periods.

In comparison to Fig. 5 stalling is not as pronounced in frequency and occurs simultaneously in both sub-networks. Neither frequency or amplitude recover fully, but both are already declining pre-stimulus. The transition phase is visible after fusing with peaks in amplitude and decline in frequency. Network-spanning contractions are occurring in the big sub-network before fusion and are directed towards fan regions and the cut site. In general, the fan size at the cut site is comparatively small and reached maximal size shortly after fusion. 


\section{S5. Supplemental Movies}

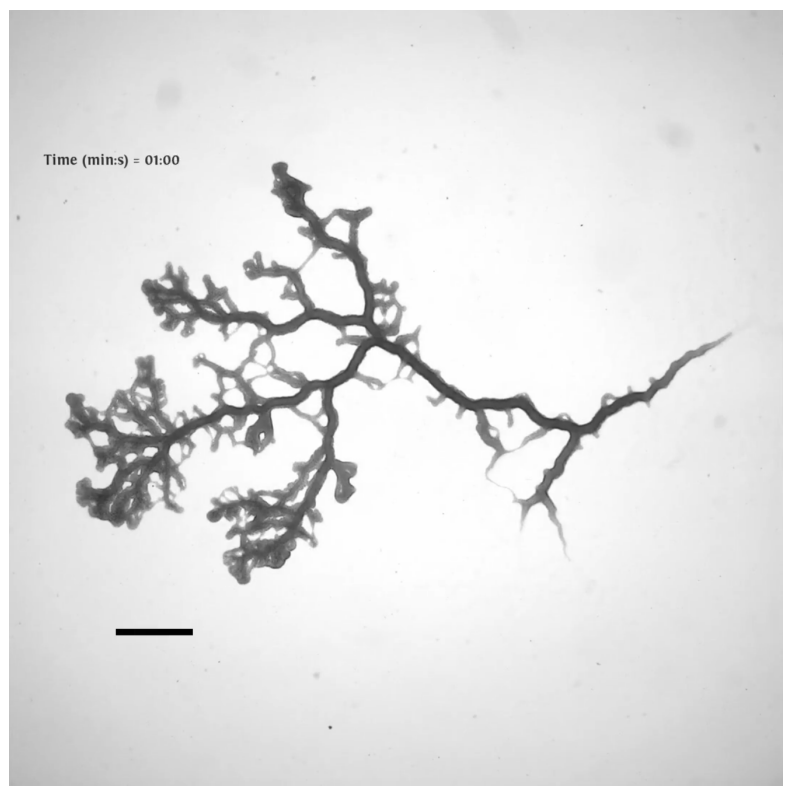

Movie S1. Bright field movie of the representative data set for wound healing. Note, that the cessation of oscillations in the top and bottom part (big and small subnetwork) is on different time scales. Scale bar $=1 \mathrm{~mm}$.

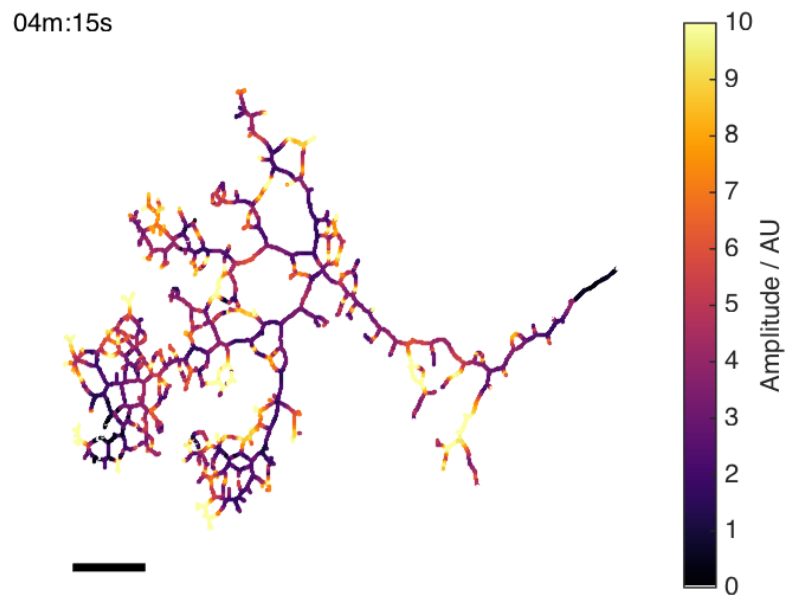

Movie S2. Movie of the oscillation amplitude spatially mapped across the network. Amplitudes below a threshold of 0.1 are omitted to visualize pruning. Due to detrending, the relative time point of cut appears shifted to 19 min and $50 \mathrm{~s}$. Scale bar $=1 \mathrm{~mm}$. 


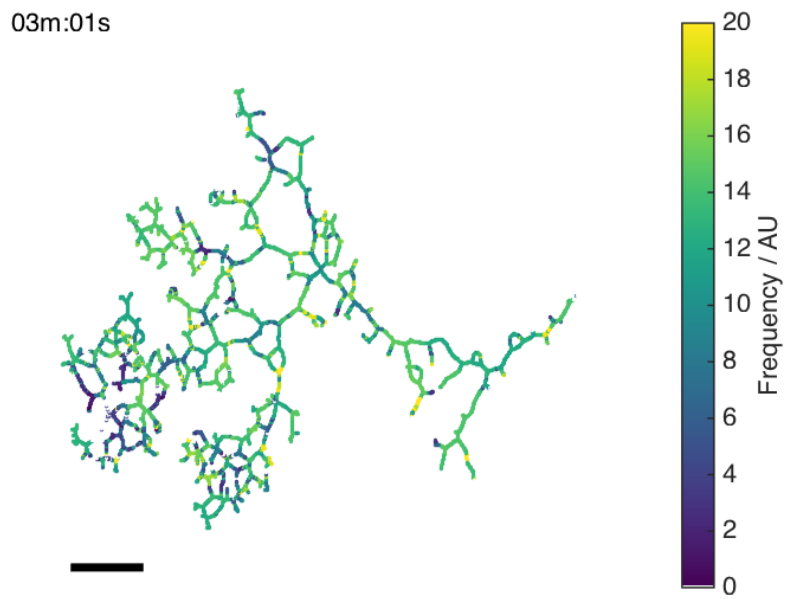

Movie S3. Spatially mapped oscillation frequency across the network. Mind that due to detrending, the relative time point of cut appears shifted to $19 \mathrm{~min}$ and $50 \mathrm{~s}$. Scale bar $=1 \mathrm{~mm}$.

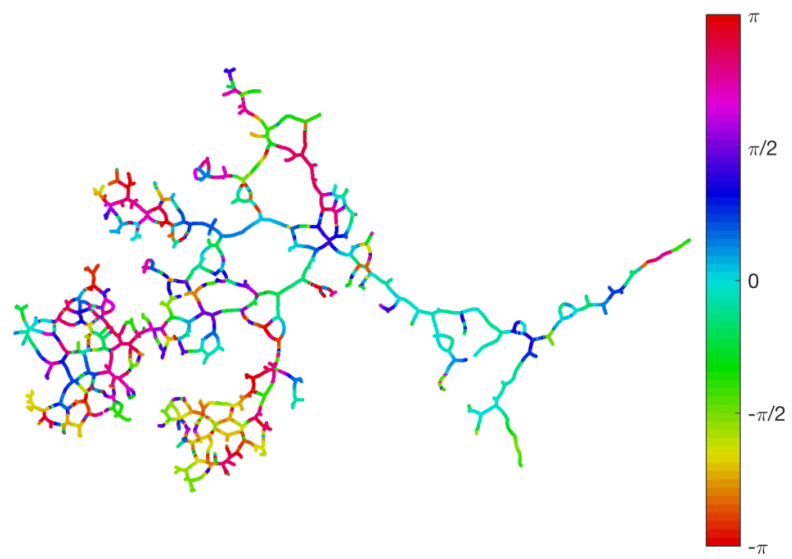

Movie S4. Spatially mapped phase pattern across the network with additional Gaussian smoothing in a 30px environment. Cut time is at $19 \mathrm{~min}$ and $50 \mathrm{~s}$. Mind change in phase waves from before cut, during stalling and during correlated phase. The cut itself is not visible in the phase pattern due to low amplitude residual slime and spatial smoothing. It is recommended to follow a single colour over a time span to guide ones eye. The network spans maximal $8.4 \mathrm{~mm}$ along the $\mathrm{x}$-axis. 


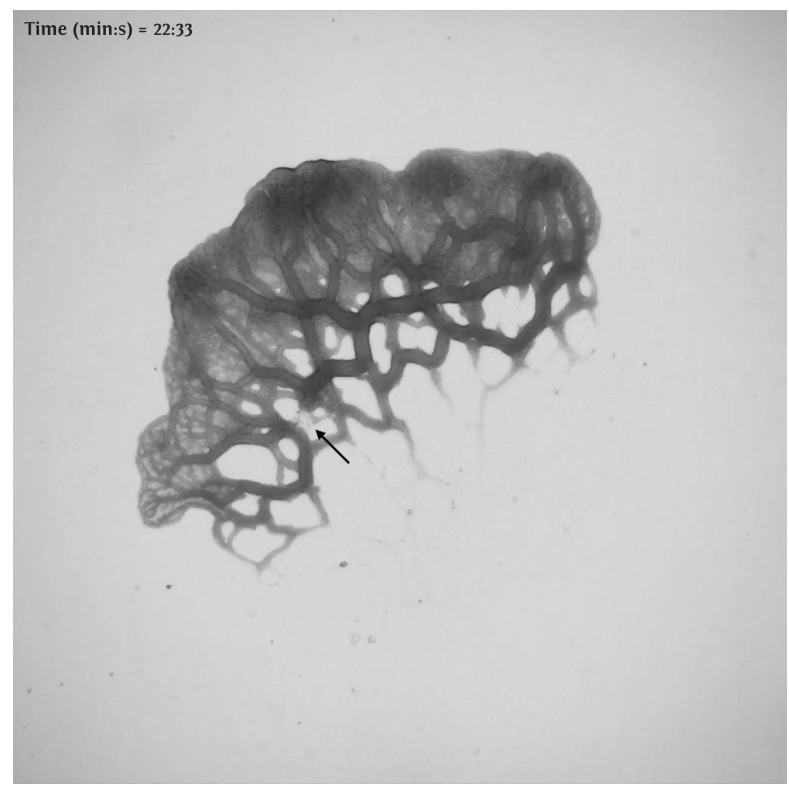

Movie S5. Bright field movie of E1 dataset showing severe stalling. Exemplary dataset for missing network morphology which makes network-based quantitative analysis impossible. Arrow marks cut location. Mind change in oscillation pattern, morphology and fan growth right after cut. Scale bar $=1 \mathrm{~mm}$. 


\section{E1. List of experiments}

This section provides a complete list of acquired data sets.

Each experiment is a figure with two sub-figures displaying: a) the raw images of the plasmodium at the beginning of the experiment (left), immediately after the cut is performed (middle), and at the end of experiment (right). The cut site is marked with a red cross and the section of the network chosen for kymograph analysis is marked with a red line. The scale bar always represents $1 \mathrm{~mm}$ and is shown in the latest image. b) shows the results of kymograph analysis. Here, the black bar, if applicable, denotes stalling.

The figures are complemented with a detailed descriptions of the morphological dynamics based on visual inspection of the bright field data. Both quantification and visual inspection together allow to identify if stalling does happen or not as discussed for each data set.

The experiments are sorted in a descending order by the magnitude of stalling. The organisms in experiments from Fig. E1 to Fig. E7 show most pronounced stalling, followed by a weaker display of stalling from Fig. E8 to Fig. E15. The organisms in experiments from Fig. E16 to Fig. E21 do not show a clear stalling behaviour. 


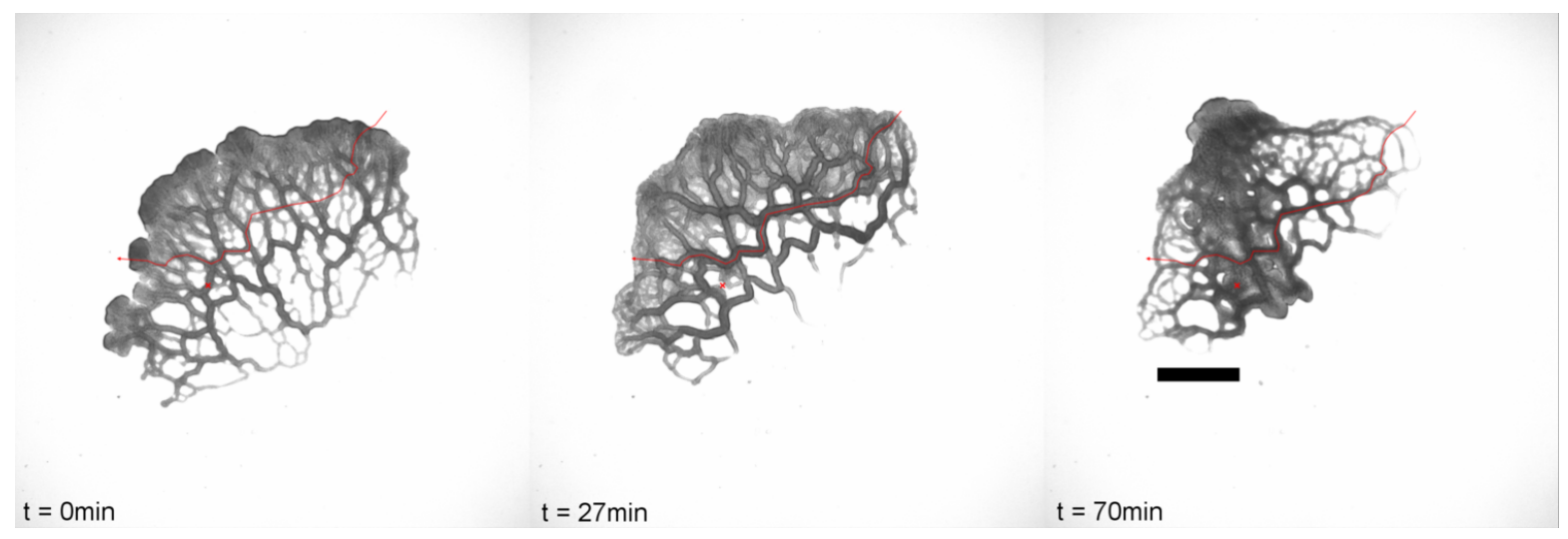

(a)

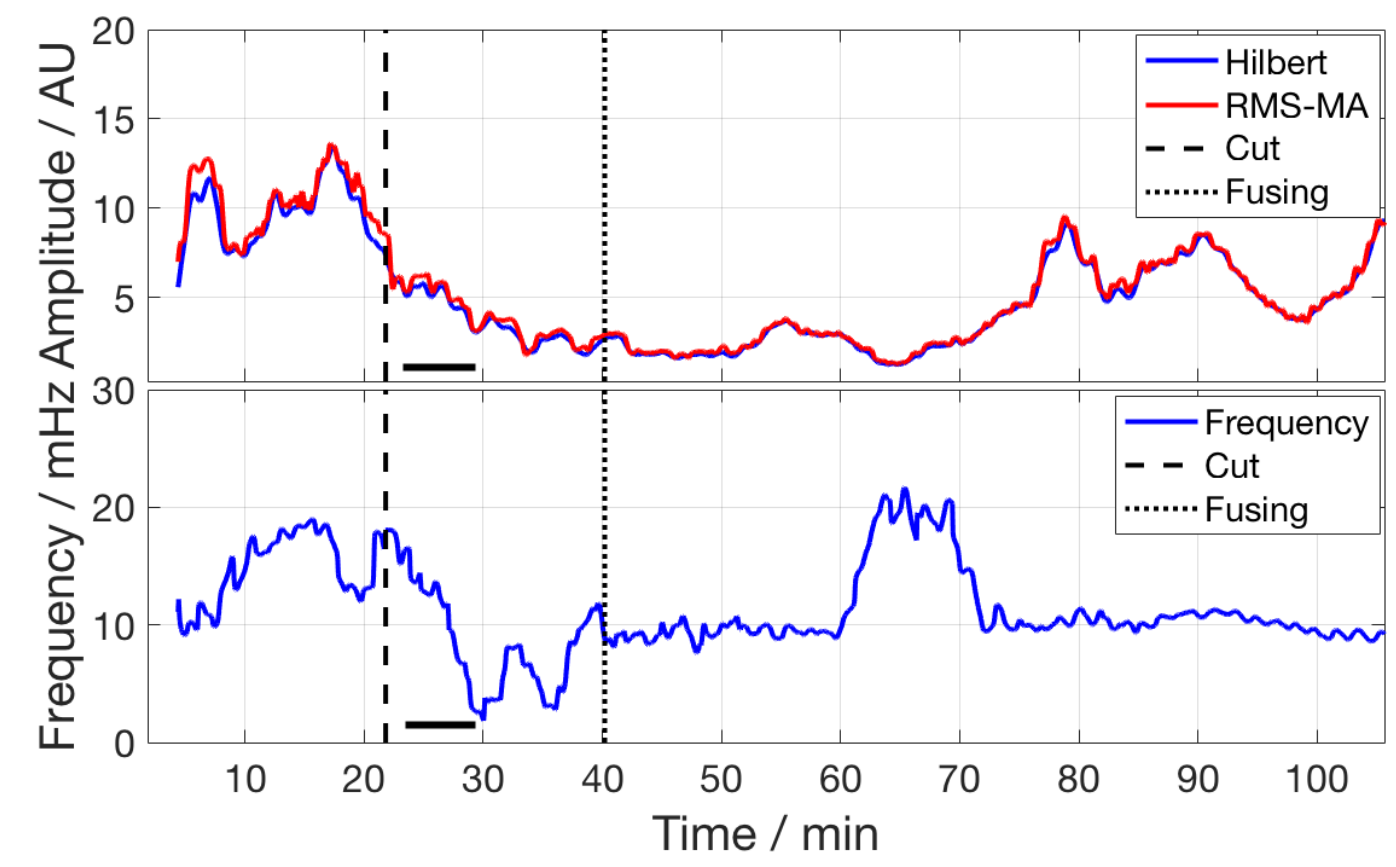

(b)

Figure E1: A small cut is performed across two of the thicker tube in this branched network, separating it into a large and small sub-network. A fan is first built around the cut site, then disintegrates as the flow is re-established. After an initial delay, the organism experiences stalling. 


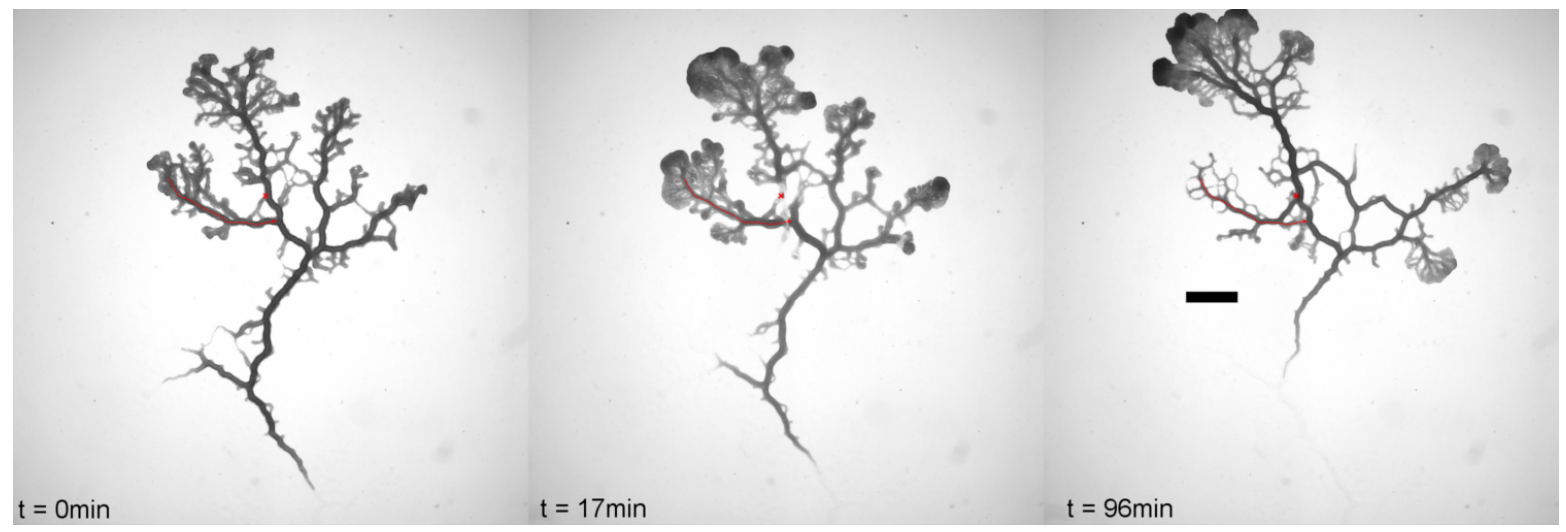

(a)

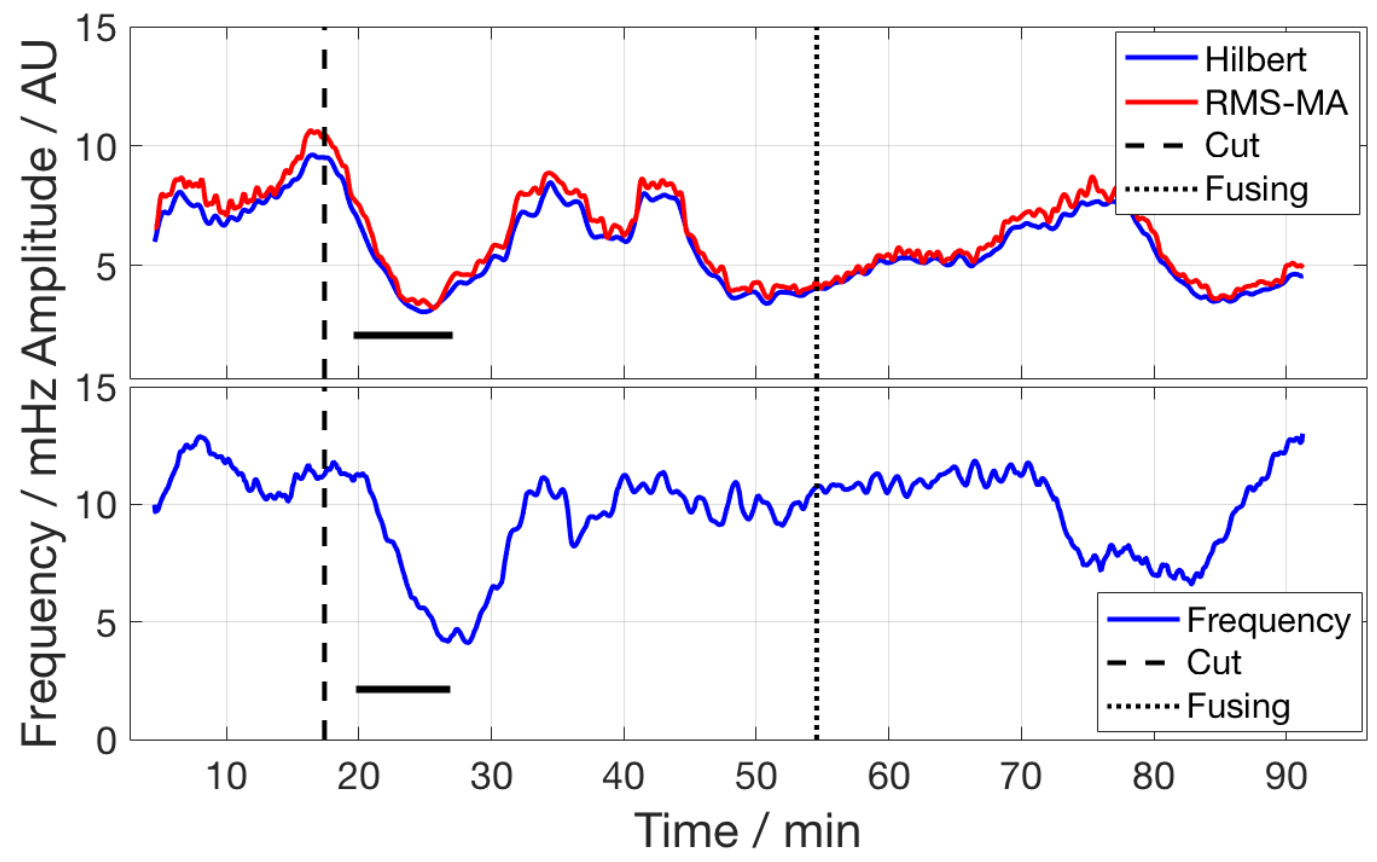

(b)

Figure E2: This data set is prominently described within the main text. We refer to the results section (3) for detailed description. 


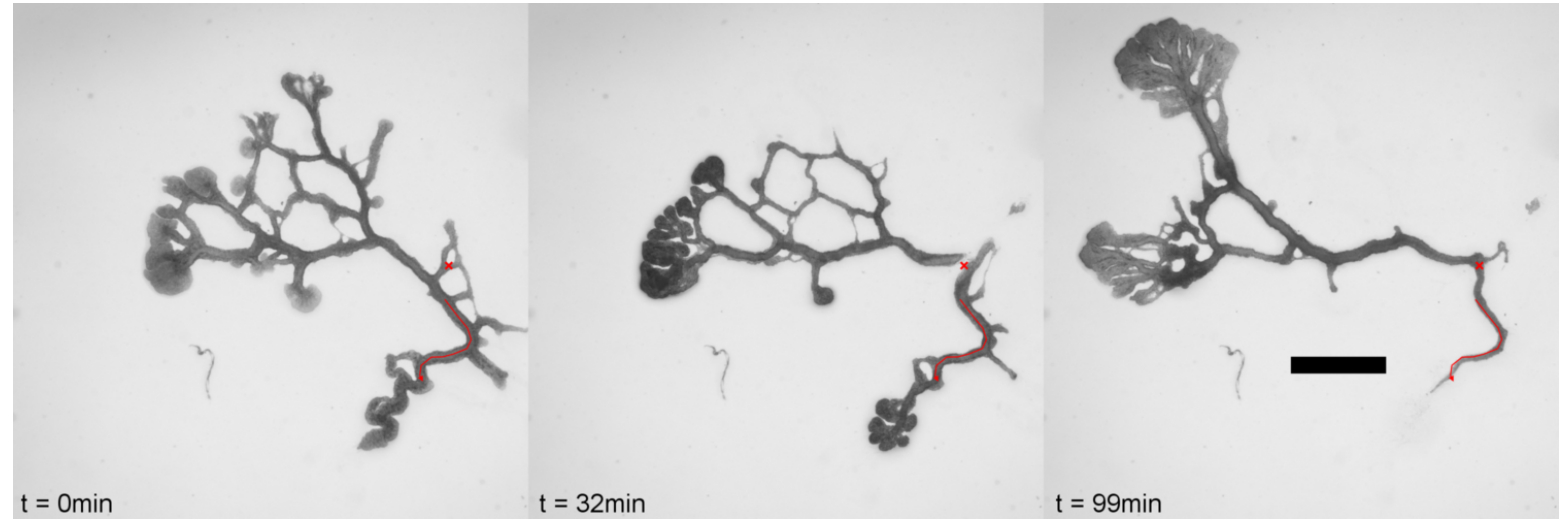

(a)

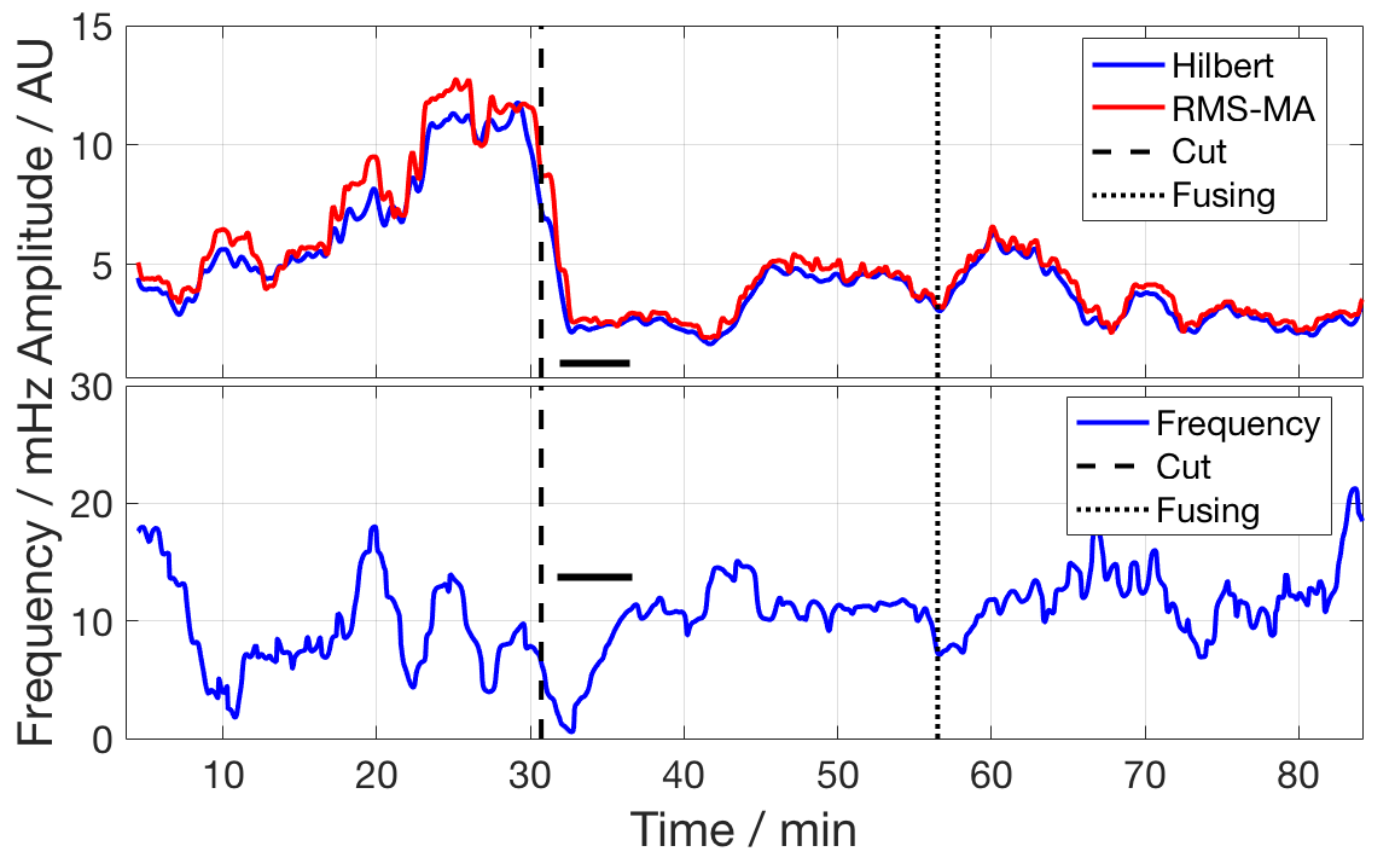

(b)

Figure E3: This small plasmodium with few tubes was cut across the thickest tube connecting the two fan regions. The cut removed a part of the tube and hence completely separated the two regions. Stalling is immediate after the cut, seen as a drop in amplitude and frequency. As the cut ends rejoin, a fan is built around the cut side. This experiment was fully analysed with quantitative analysis (see Fig.S5). 


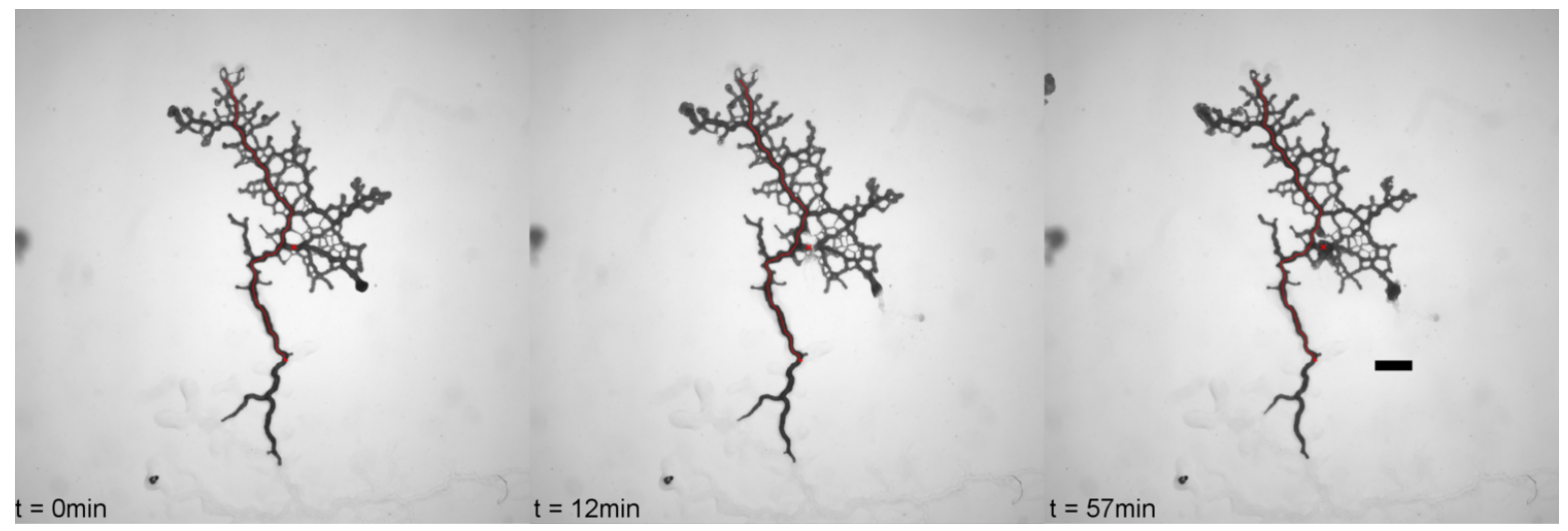

(a)

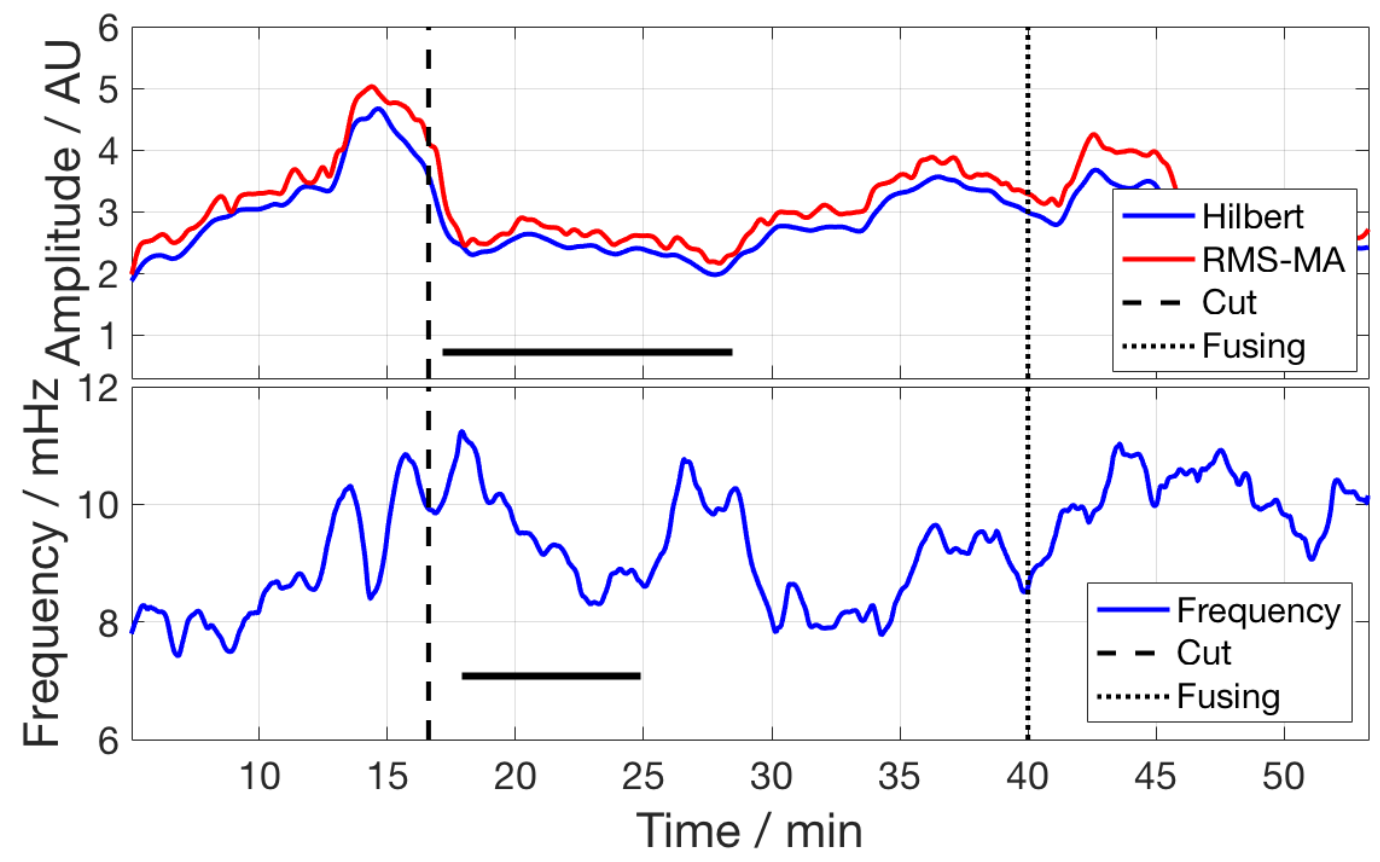

(b)

Figure E4: In this larger network, a thicker side tube was cut. The cut slightly separated the ends of the tube which then rejoin while a large fan is built around the cut site. The organism experiences stalling, visible as a decrease in frequency and amplitude of the oscillations. The fan around the cut site maintains its size until the end of the experiment. 


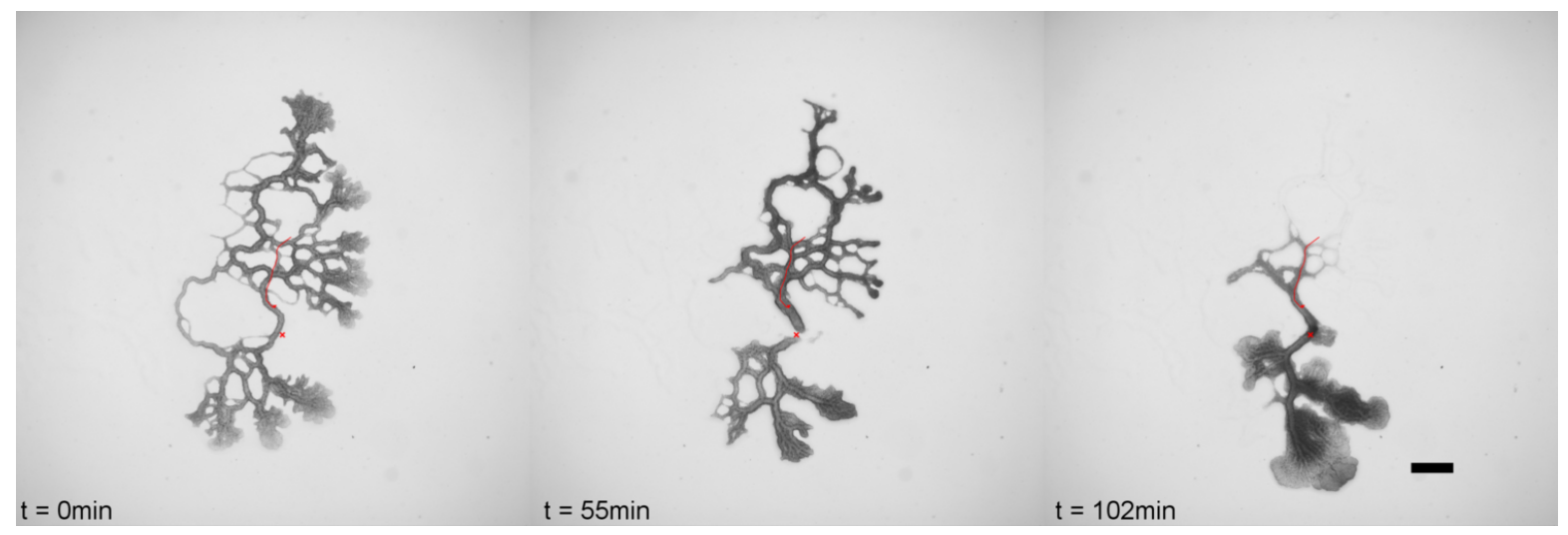

(a)

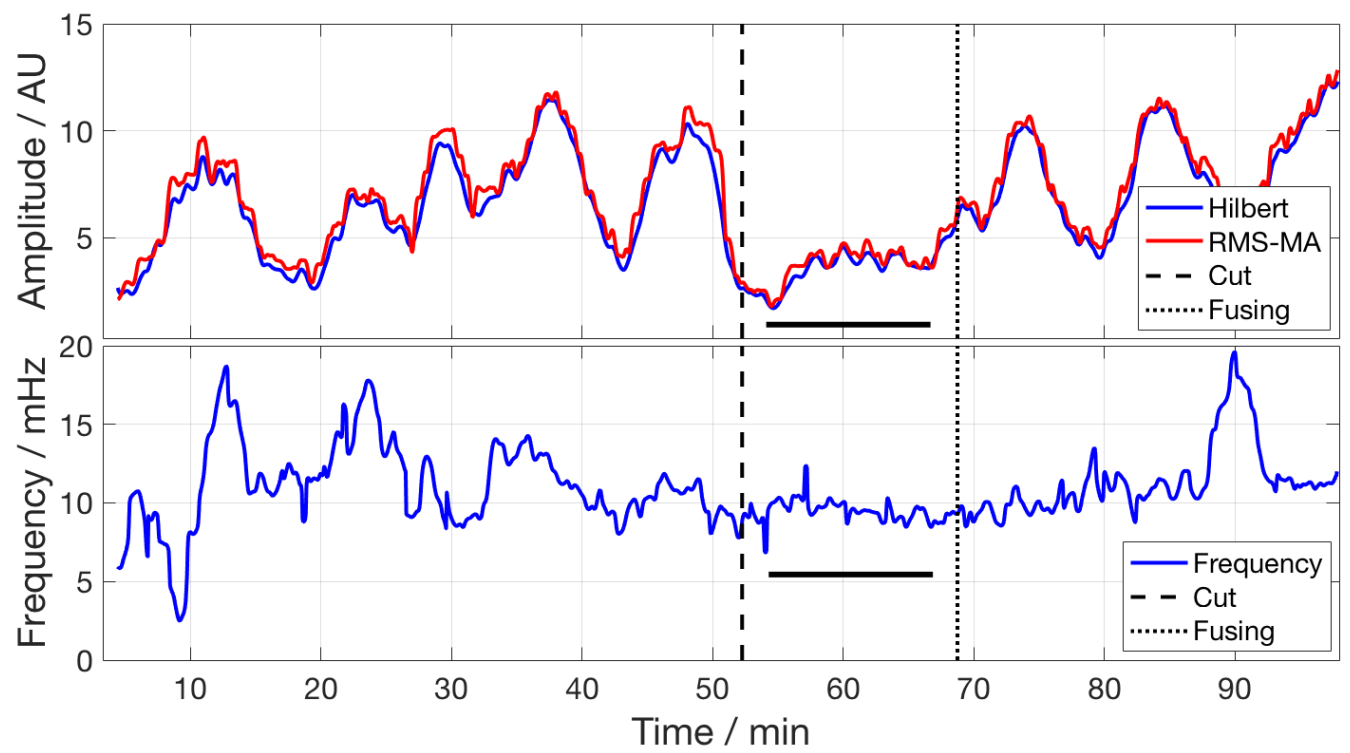

(b)

Figure E5: A cut was performed across a thick tube connecting two distant regions of the organism of comparable size, chosen such that the organism has no possibility of re-routing the flow through a close-by neighbouring tube. However, the cut barely separated the tube ends and they rejoin quickly. A fan is growing around the cut site. The stalling in both sub-networks appears in connection with very low variation both in amplitude and frequency of the oscillations compared to the pre-cut and post-rejoining phase. 


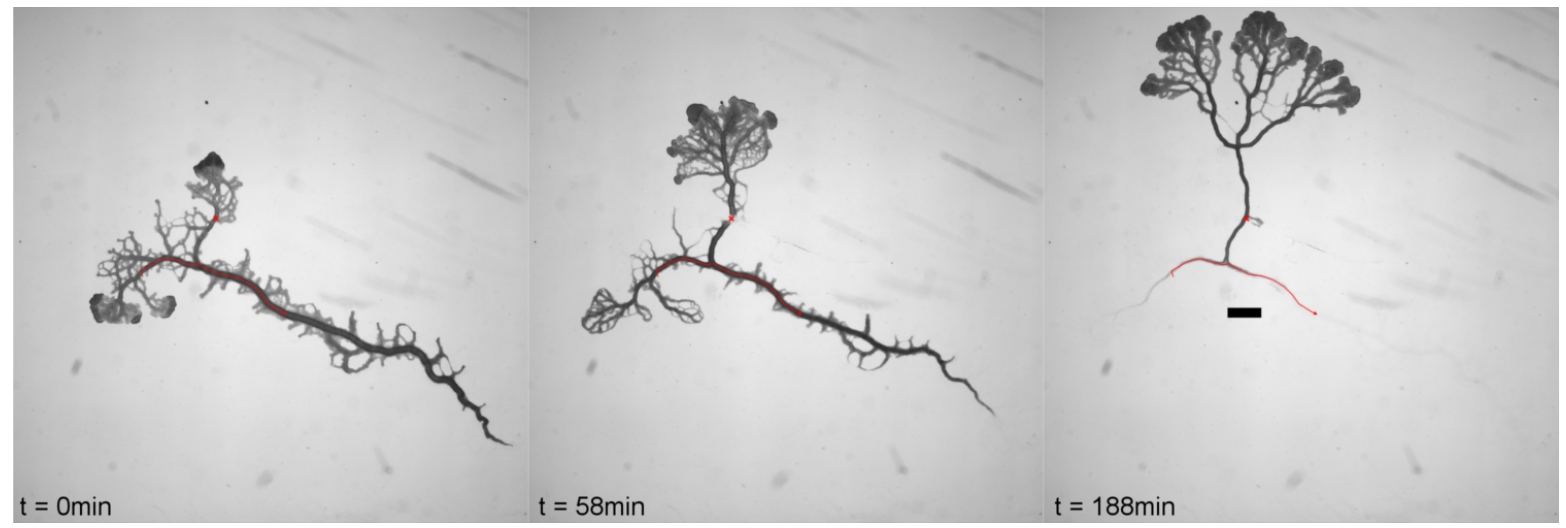

(a)

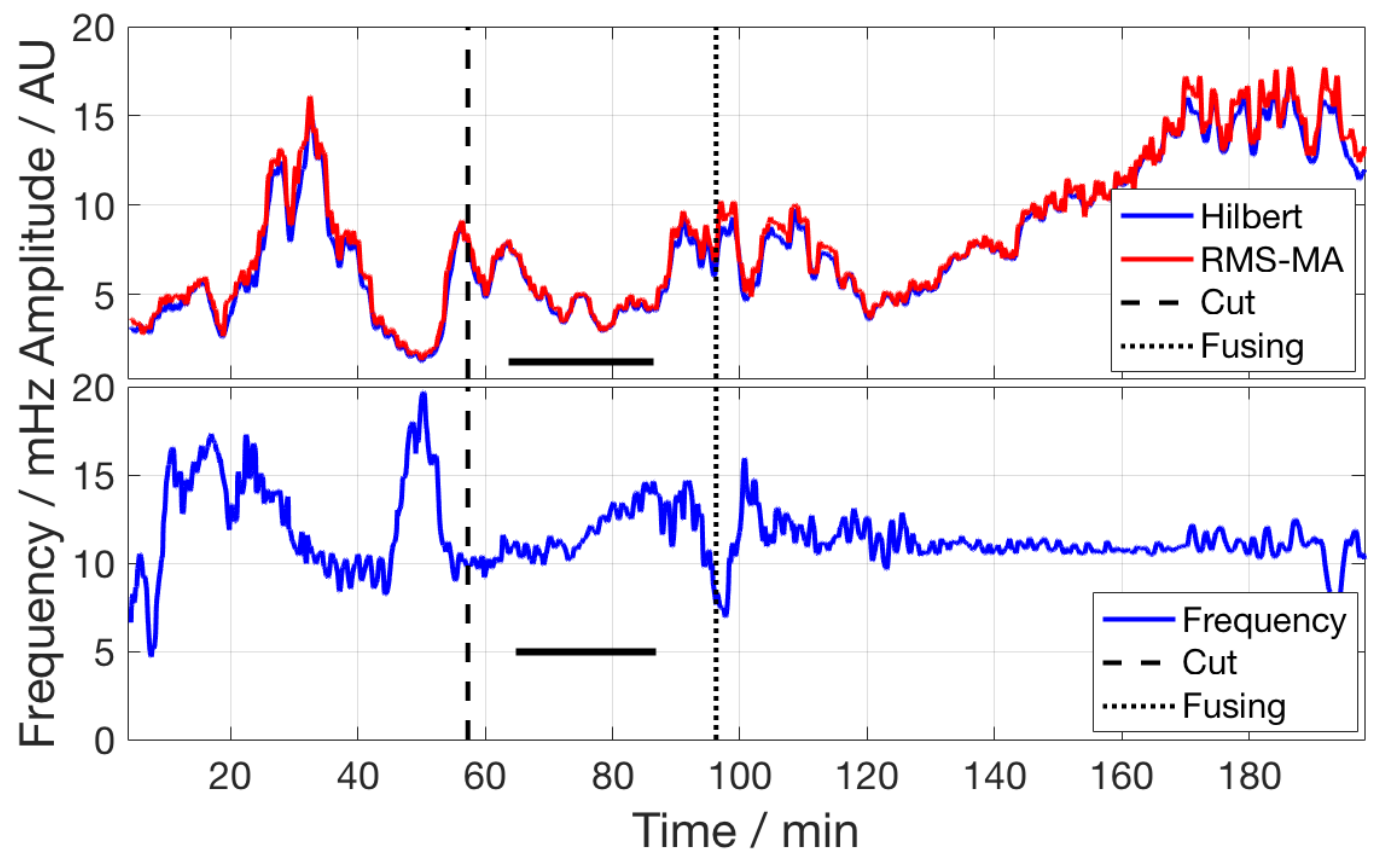

(b)

Figure E6: A cut is performed across a thick single tube connecting a large fan region with the rest of the organism. The amplitude and frequency of the oscillations stall first, followed by a gradual increase as the network rejoins the cut parts. Once the flow is re-established, the plasmodium prunes one side of the body and moves as a whole in the direction of the large fan. 


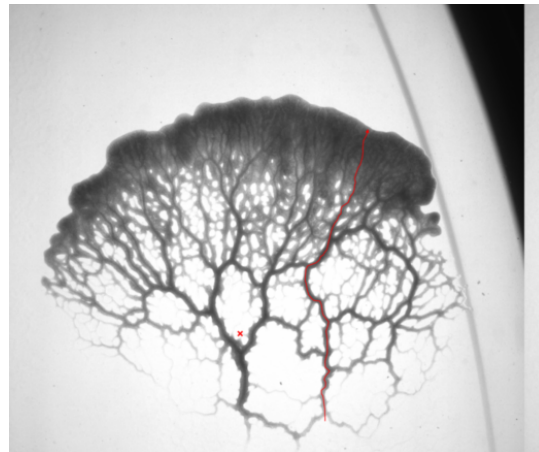

$\mathrm{t}=0 \mathrm{~min}$

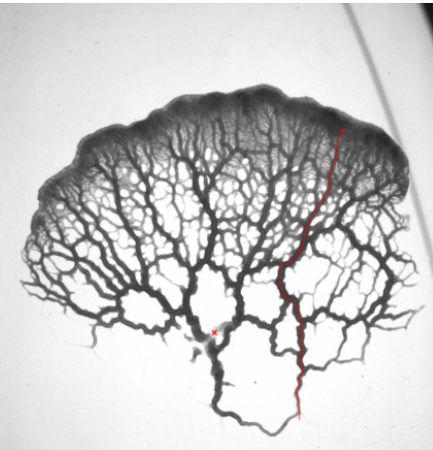

$\mathrm{t}=20 \mathrm{~min}$

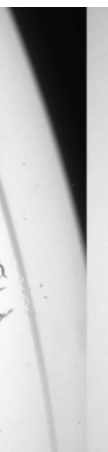

$\mathrm{t}=65 \mathrm{~min}$

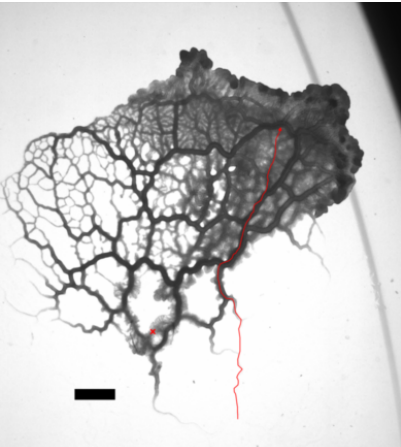

(a)

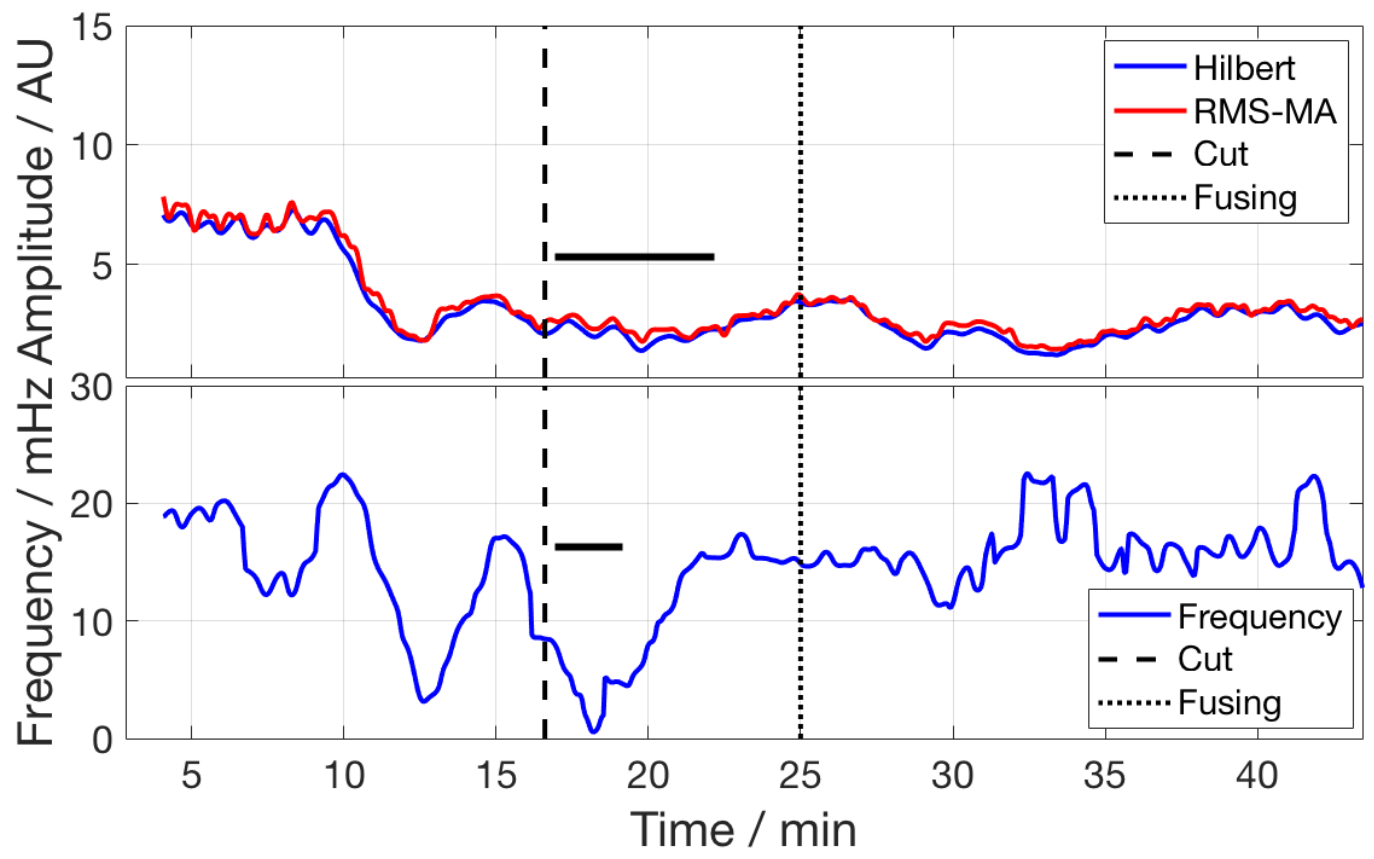

(b)

Figure E7: The morphology of this small network changes rapidly over the course of the experiment. A cut is performed across two neighbouring wide tubes at the base of the network. The tube segments fuse back completely as fans grow around it. Stalling is very prominent in this experiment and shows as a large drop in the frequency of the oscillations. 


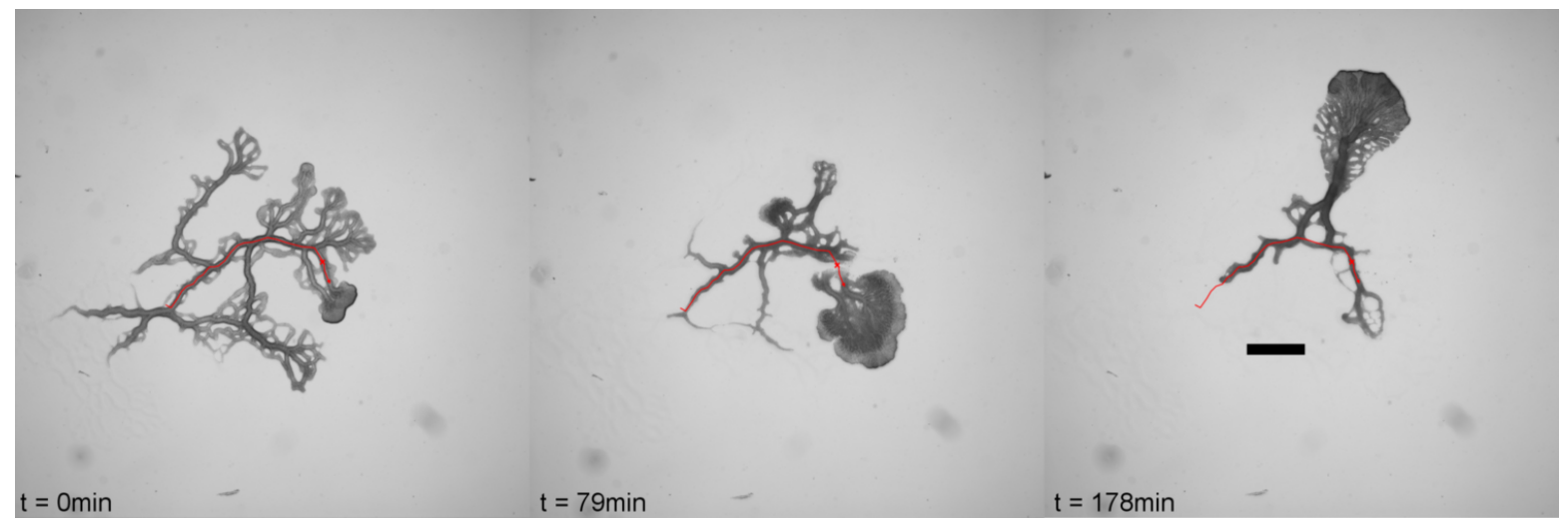

(a)

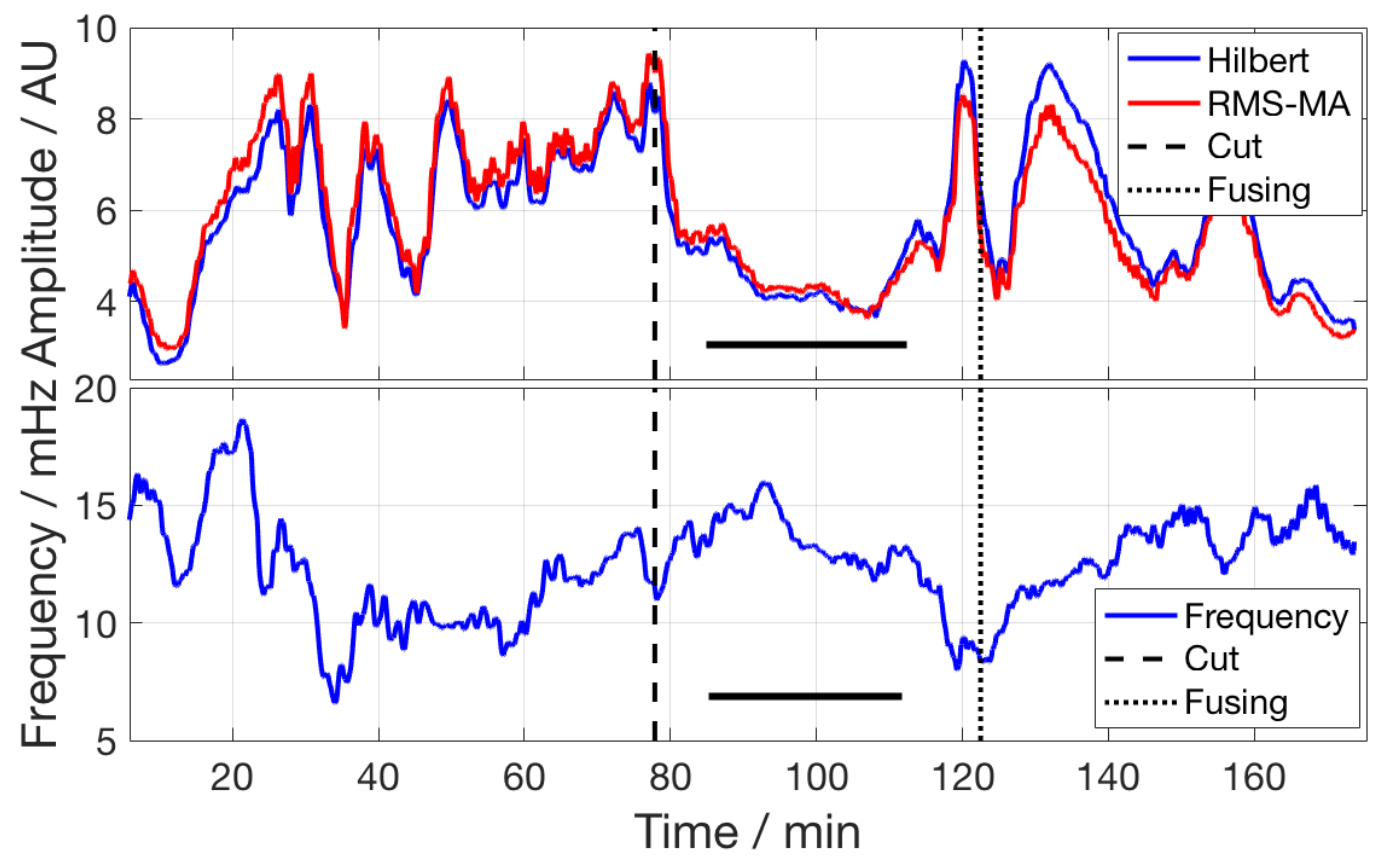

(b)

Figure E8: A cut is applied across the base of the only large fan in the network, resulting in damage of two thick tubes. The large fan, now separated, stalls and then builds body mass at the cut site. After rejoining, the organism as a whole builds a large fan and moves as a whole. 


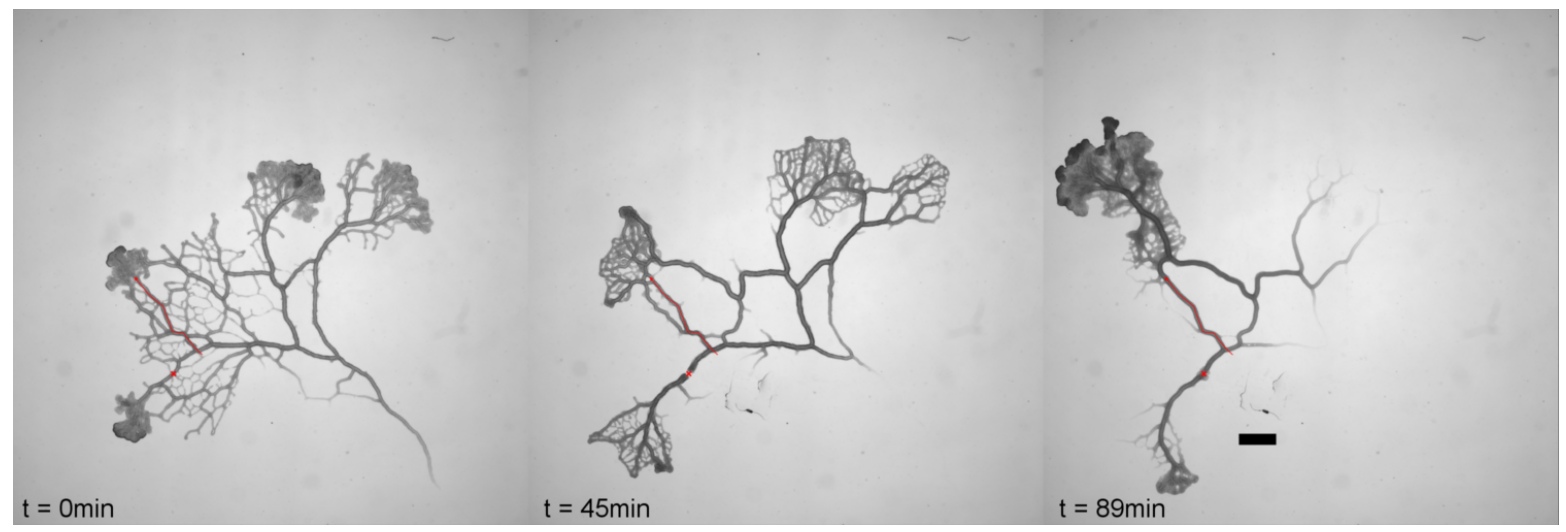

(a)

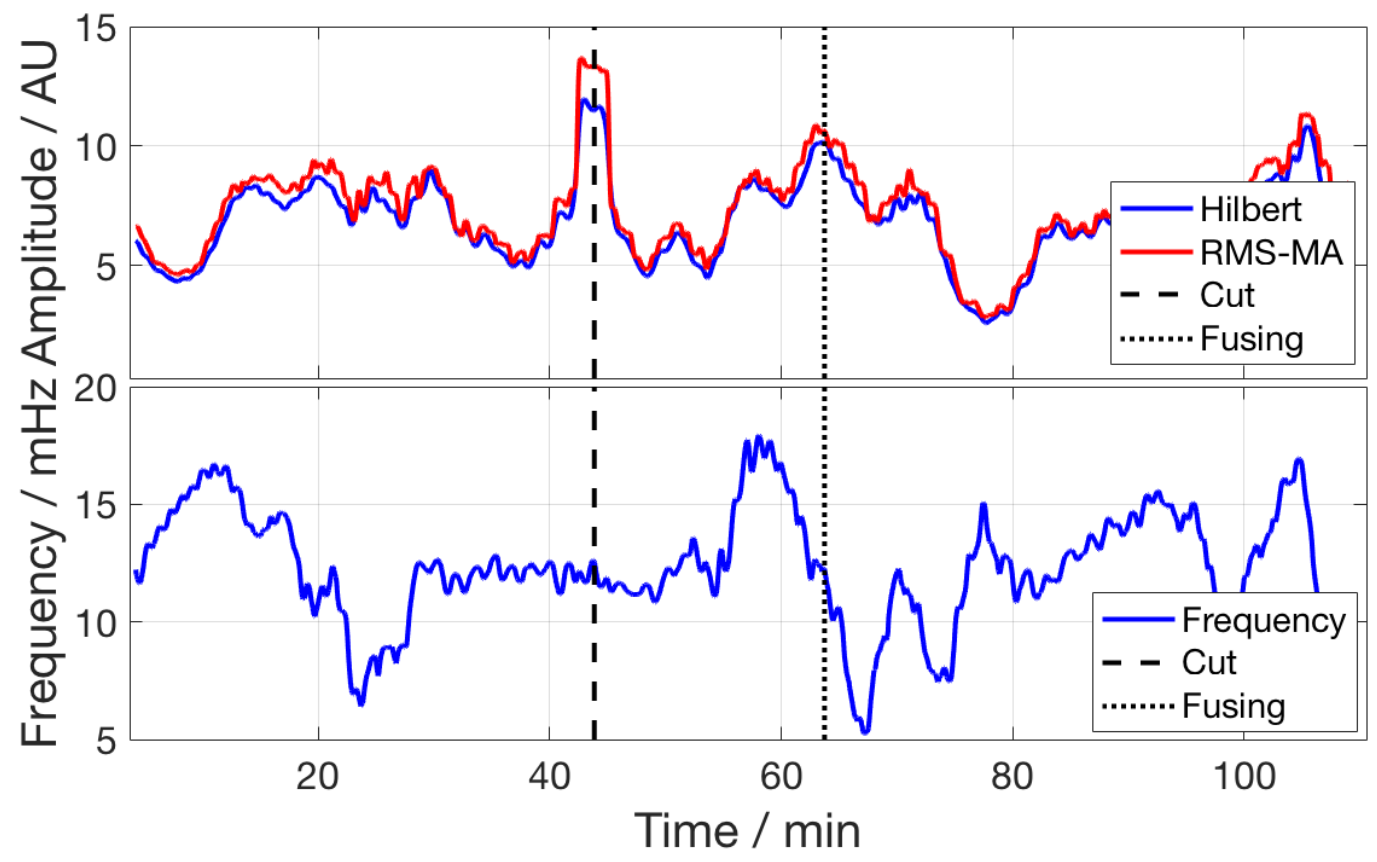

(b)

Figure E9: Here, four fan regions are initially growing and one of these is cut off at the only connection. The cut site is repaired fairly quickly with minimal fan growth. The amplitude and frequency hardly drop after the cut, yet fan growth in the periphery stalls and only resumes after flow is reinstated. After fusion the smaller sub-network is declining and fans are grown in the big sub-network. 


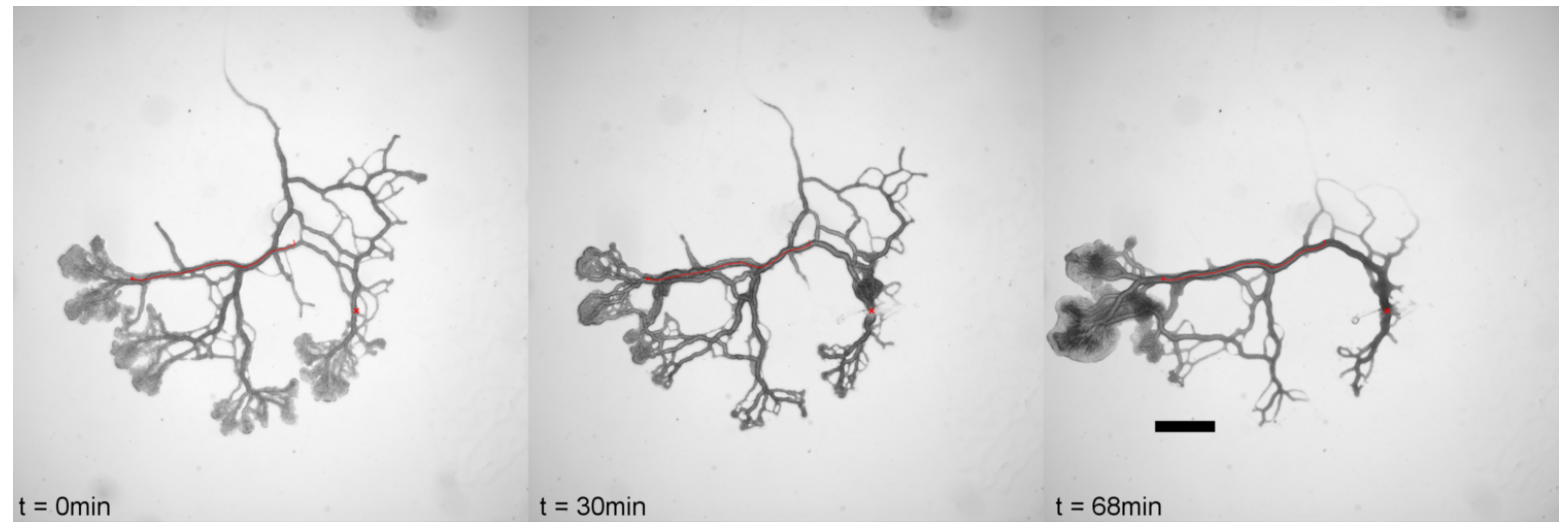

(a)

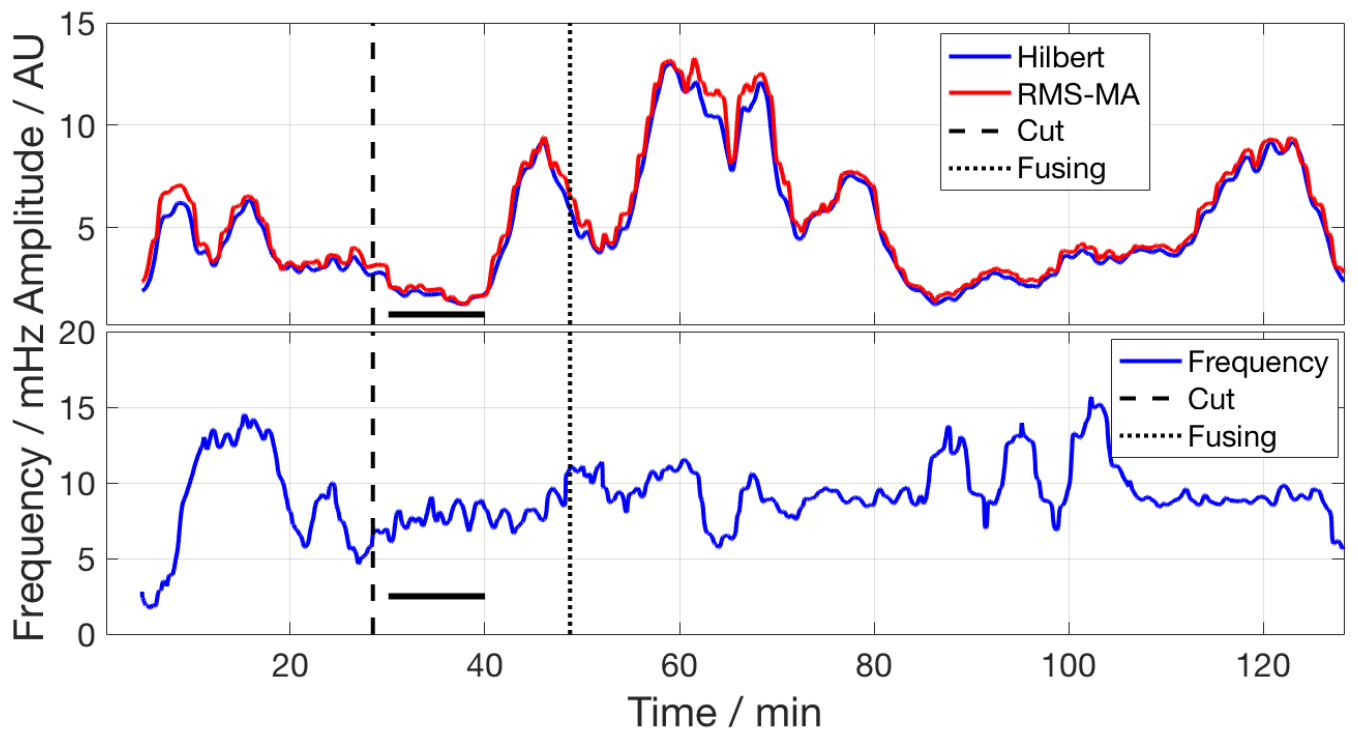

(b)

Figure E10: A cut is performed across one of the main tubes, distal from the region where most of the body mass is concentrated. The kymograph along the thickest tube shows stalling as a drop in the amplitude. A small fan grows at the site of the cut from both sub-networks. As the tubes refuse and the flow is established, the organism builds big fans and moves out of the imaging window. 


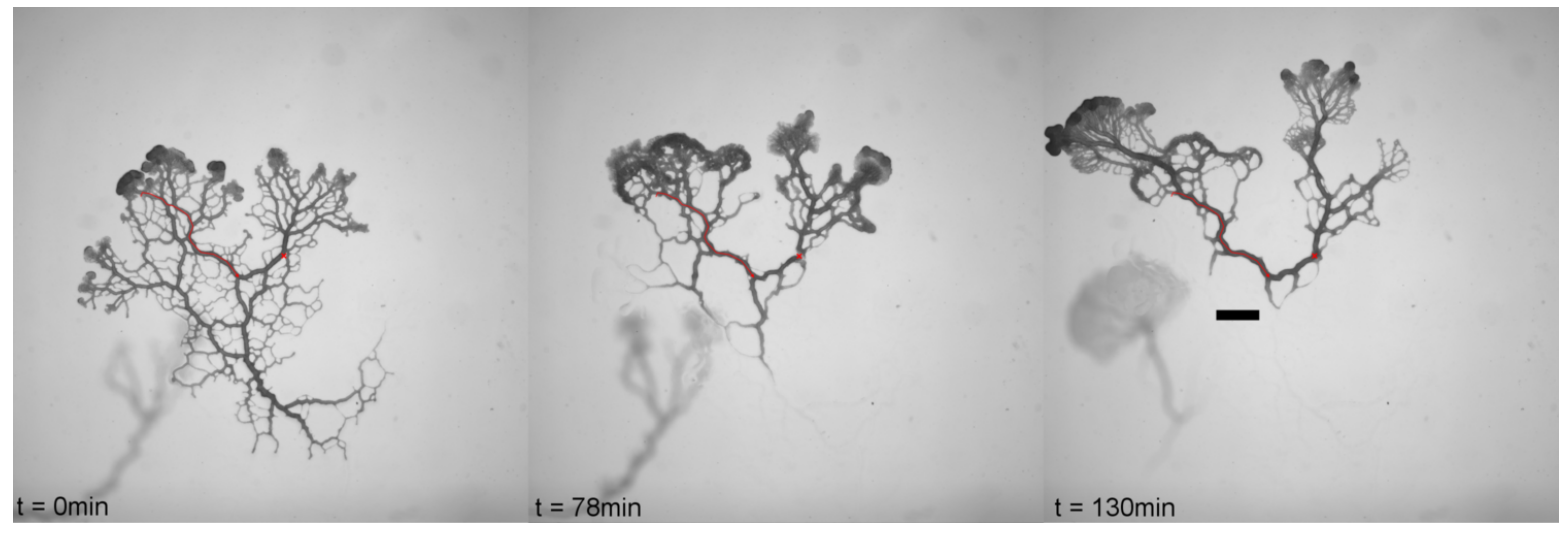

(a)

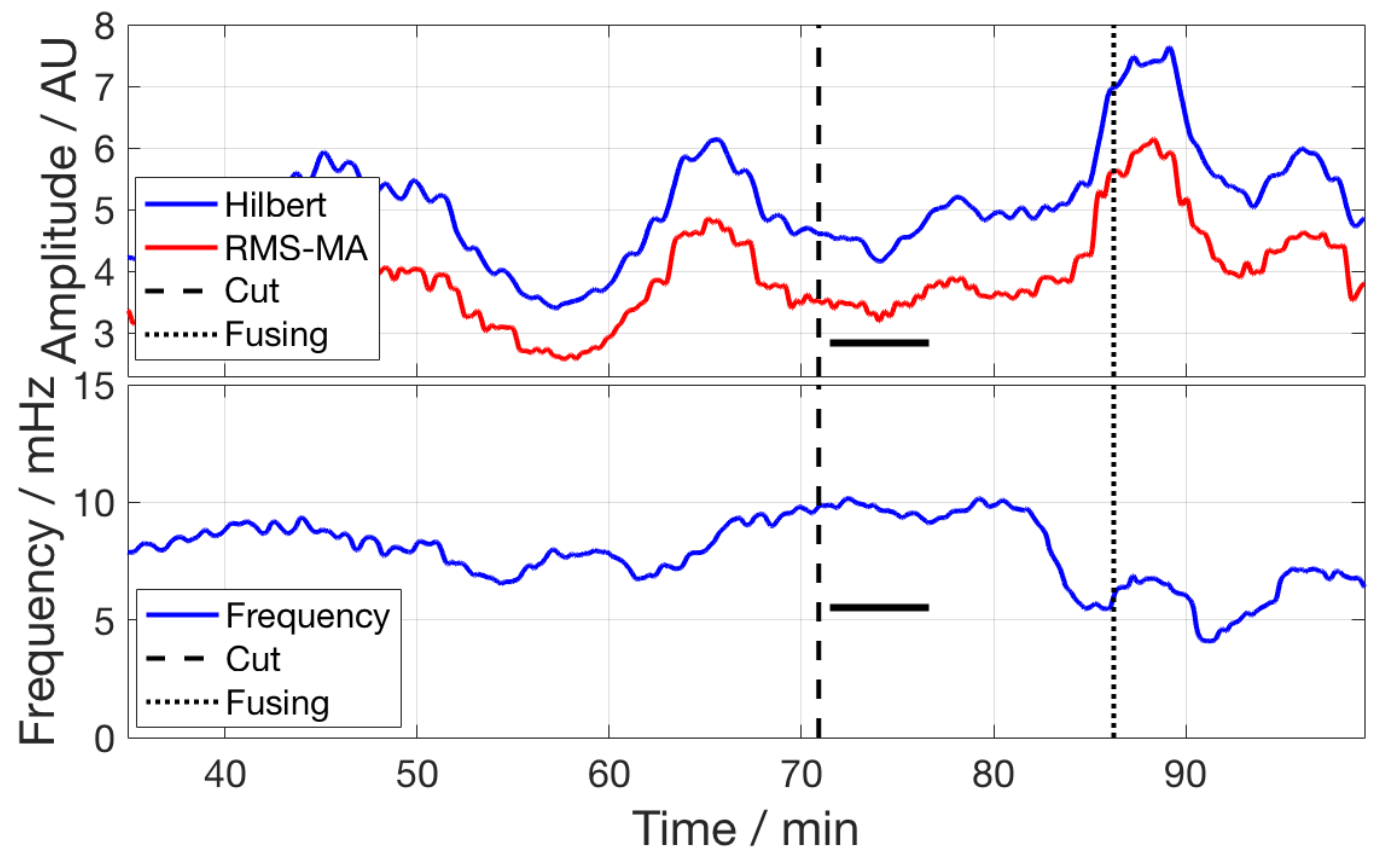

(b)

Figure E11: Initially the organism rapidly changes its morphology, likely as a response to light. A cut was performed across the biggest tube connecting the two fan regions, a site with no possibility of re-routing the flow through neighbouring tubes. The organism experiences slight stalling and quickly rejoins the cut tube ends. A small fan is built around the cut site. 


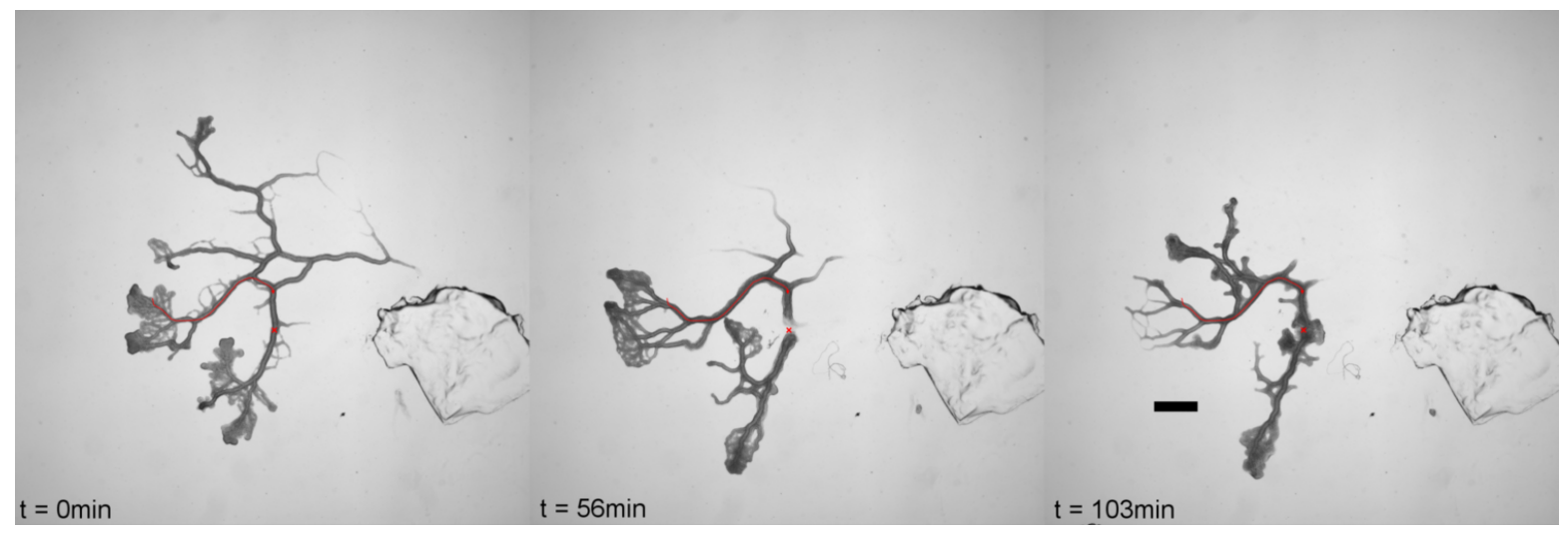

(a)

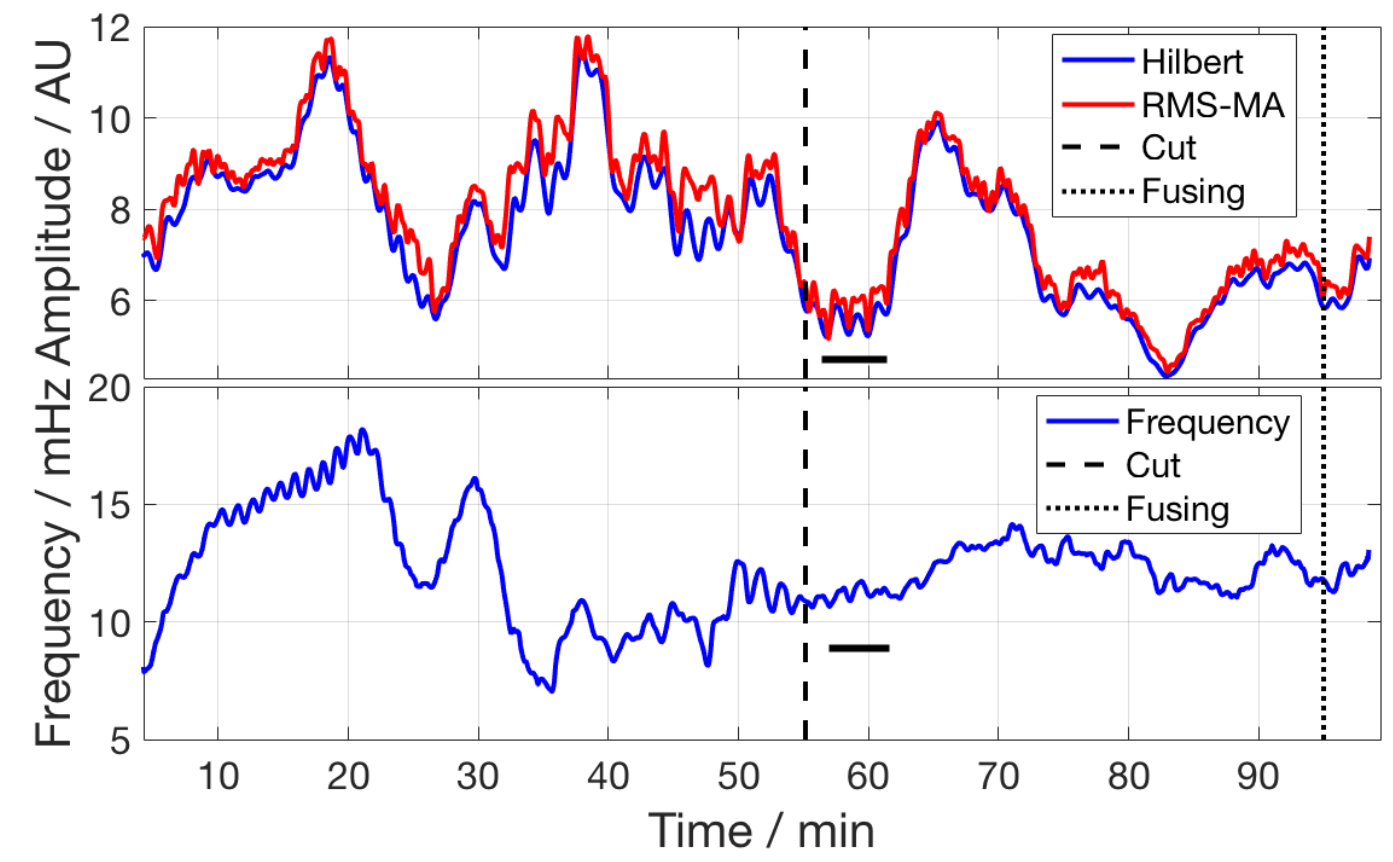

(b)

Figure E12: The organism experiences a putative light shock in the beginning indicated by the change in morphology. The cut is applied across a thick tube connecting two regions of comparable size and shape, separating them completely. The two subnetworks take a relatively long time to rejoin, meanwhile amassing new thick fans out of existing veins. Stalling is visible as a drop in amplitude. The general behaviour of this network suggests that the effects of the initial light shock persist throughout the whole experiment. 


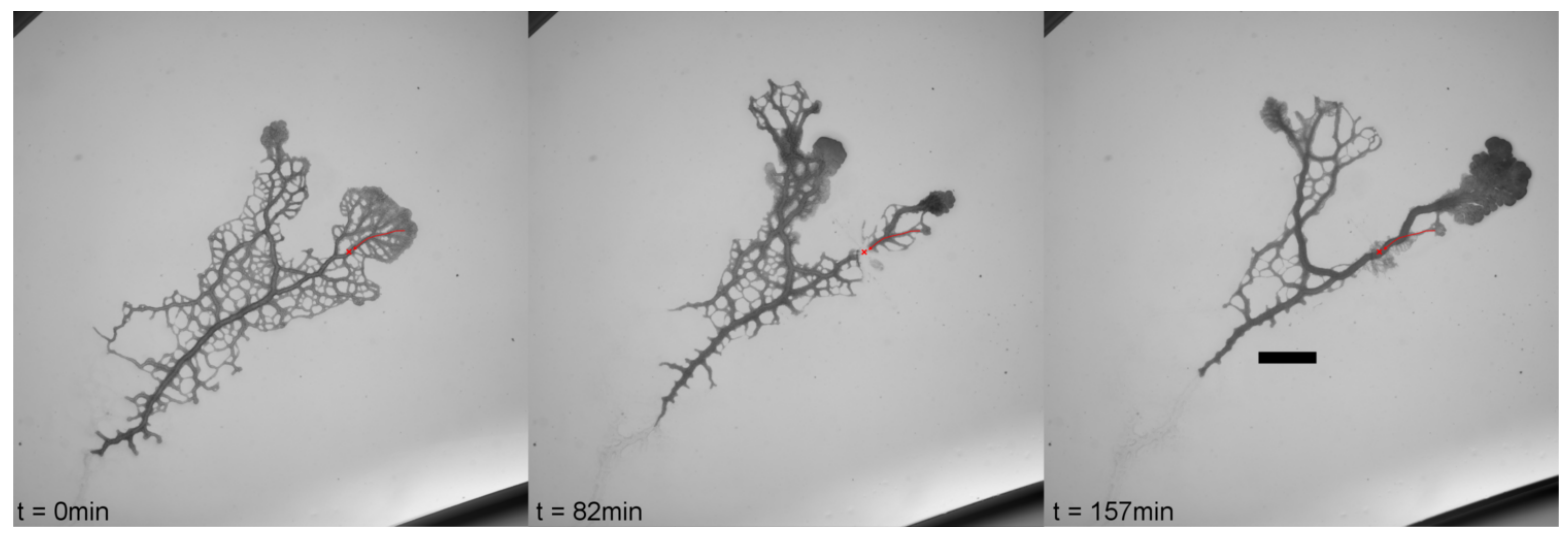

(a)

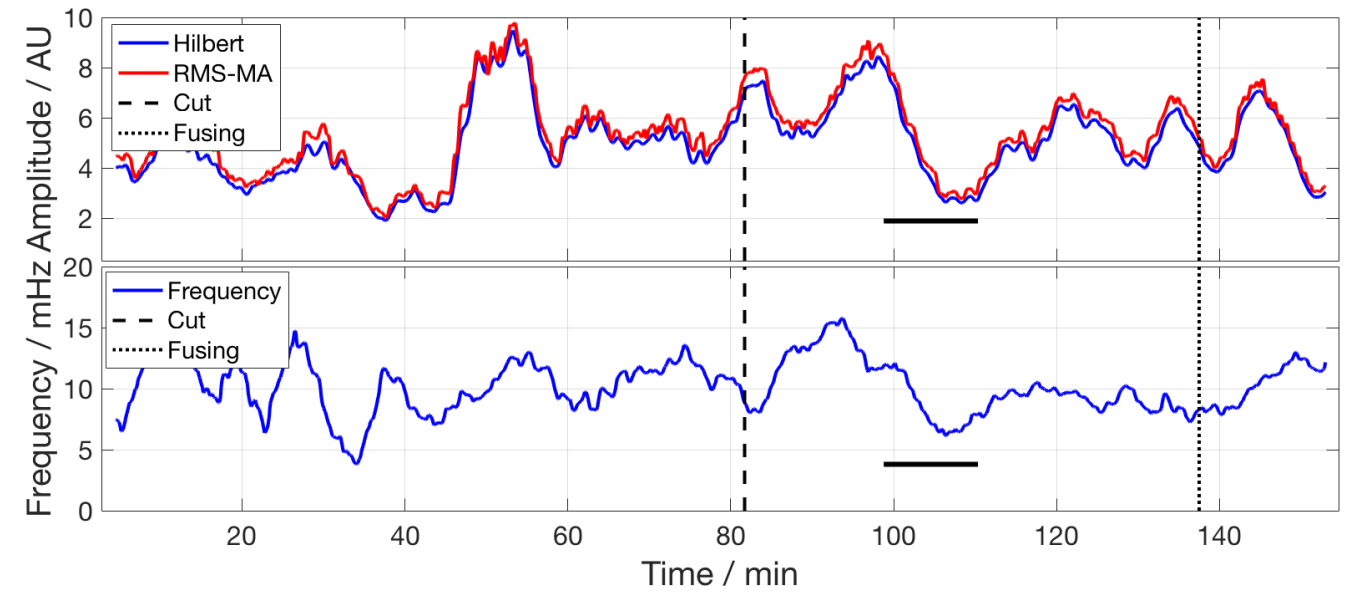

(b)

Figure E13: The cut is applied at the base of a smaller fan, followed by movement of the body mass in the fan towards the cut site. The fan in the larger sub-network retracts and the body mass moves towards the cut site. After the cut site is repaired and the sub-networks rejoin, the organism moves as a whole. The stalling occurs in both networks after the cut with a delay. 


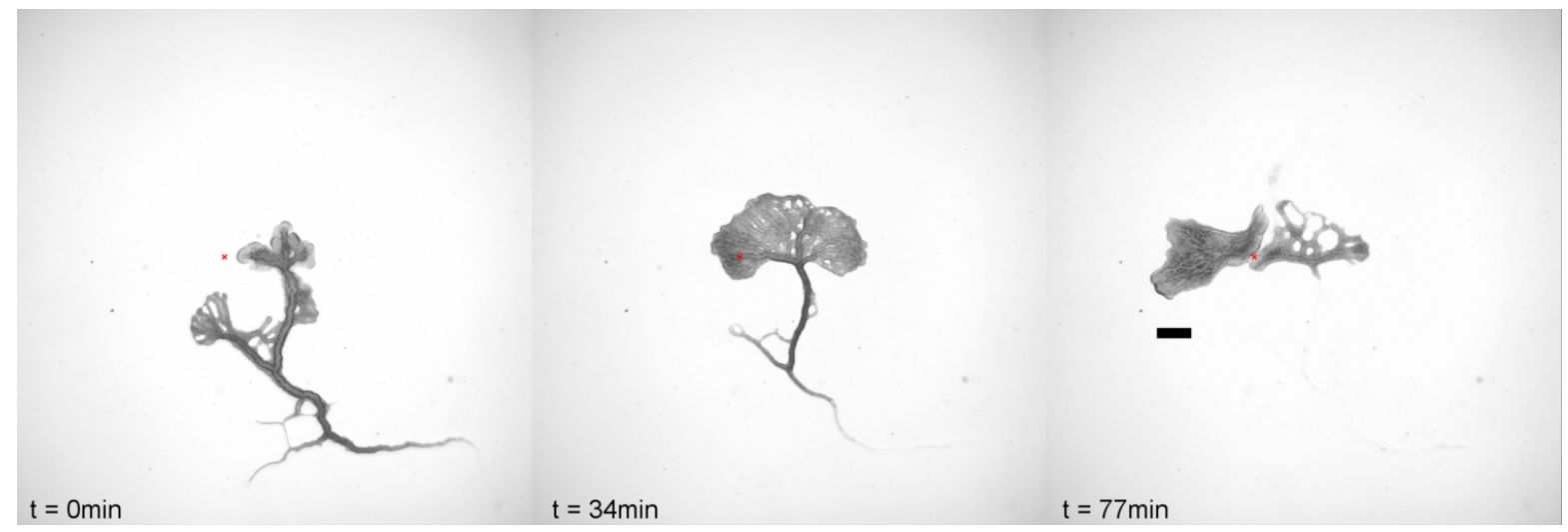

(a)

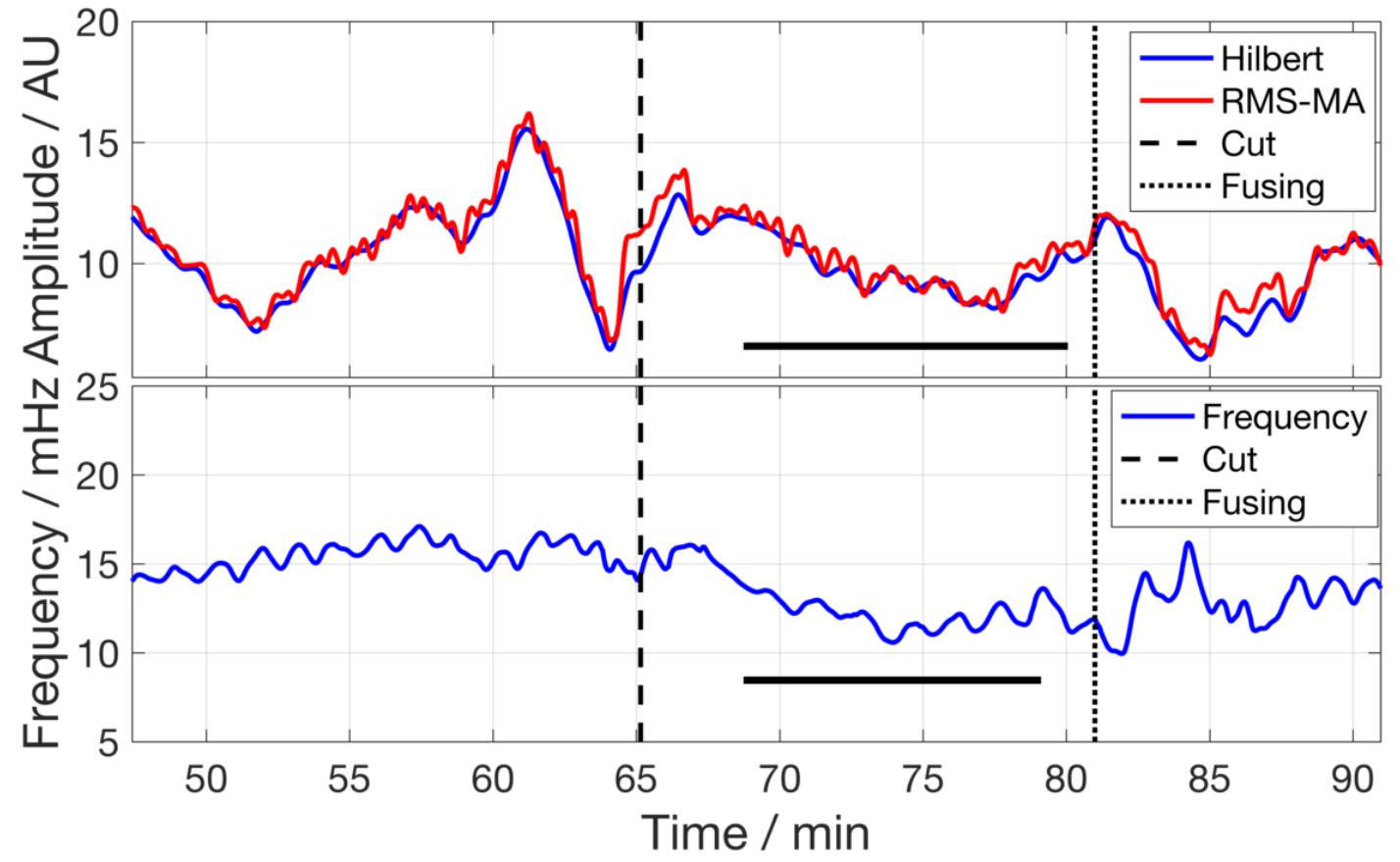

(b)

Figure E14: This plasmodium behaves rather like a very motile foraging fan than an extended network. There are hardly any hard distinctions between tube and fan in the main part of the plasmodium. The cut completely severs the specimen into two parts and stalling is evident, but it quickly returns to foraging behaviour. 


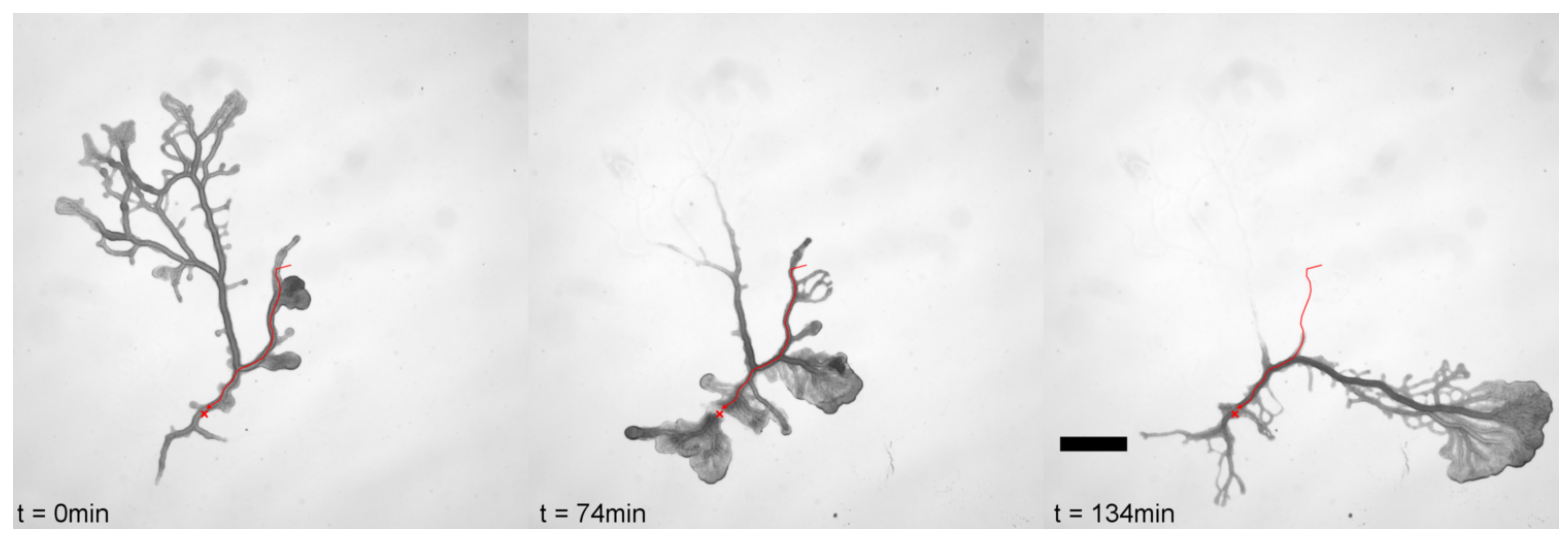

(a)

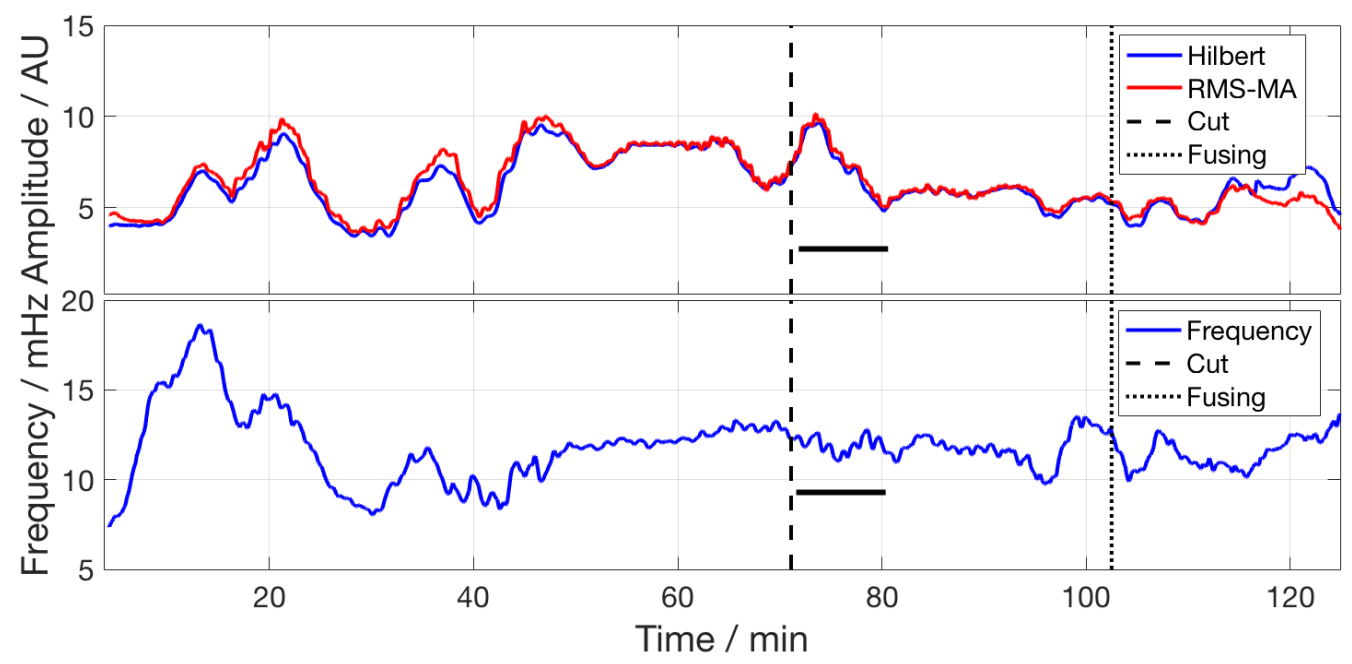

(b)

Figure E15: The network in this experiment initially shows a fast morphology change, possibly from illumination, and fan growth out of the sides of the tubes. A cut is performed along the base of a fan. The network experiences hardly any stalling, with a slight decrease in amplitude and frequency of the oscillations. The cut parts rejoin, the organism builds a large fan and moves in its direction. Is it possible that the initial effects persist during the whole experiment. 


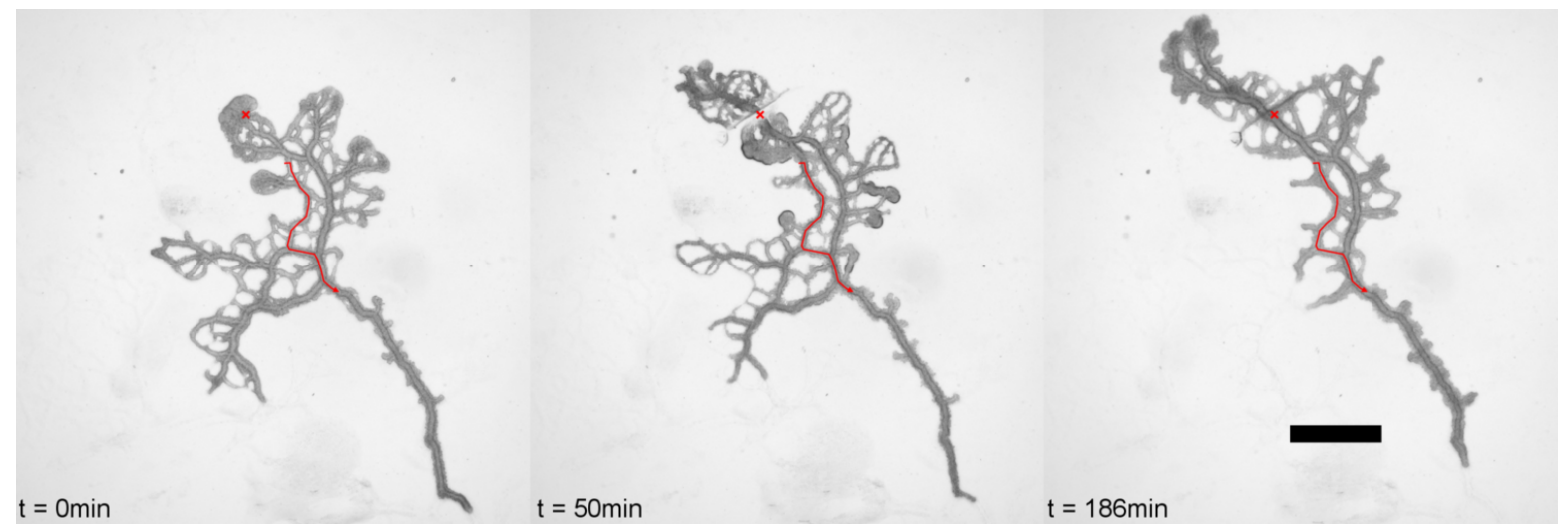

(a)

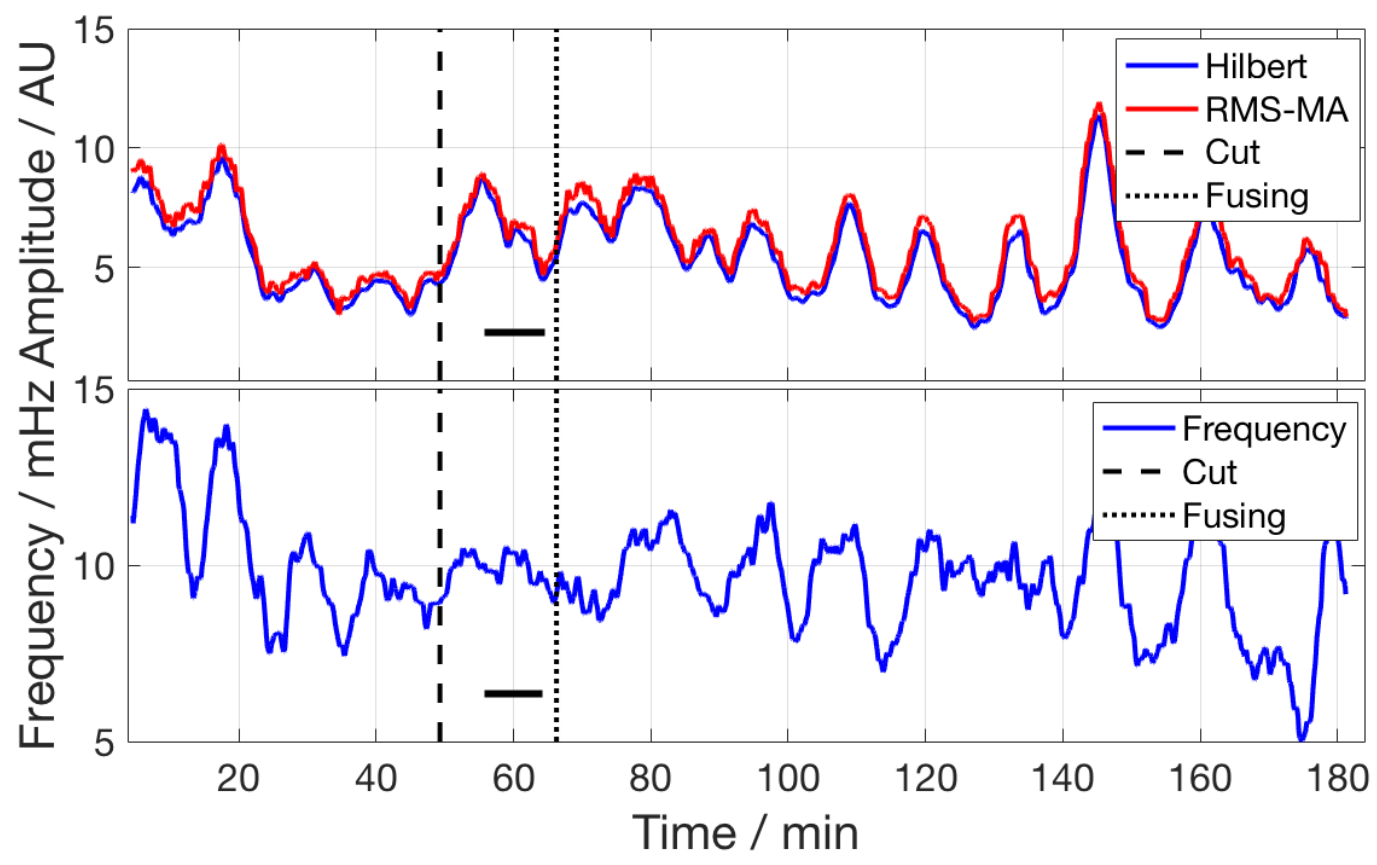

(b)

Figure E16: The plasmodium experiences a putative initial light shock which is indicated by a quick change of morphology, resulting in a network with a single fan. A cut was performed across the tube in the fan. The cut parts rejoin quickly. A thick fan is first grown at the cut site, then absorbed into the fan at the top of the image. No pronounced stalling is visible. 


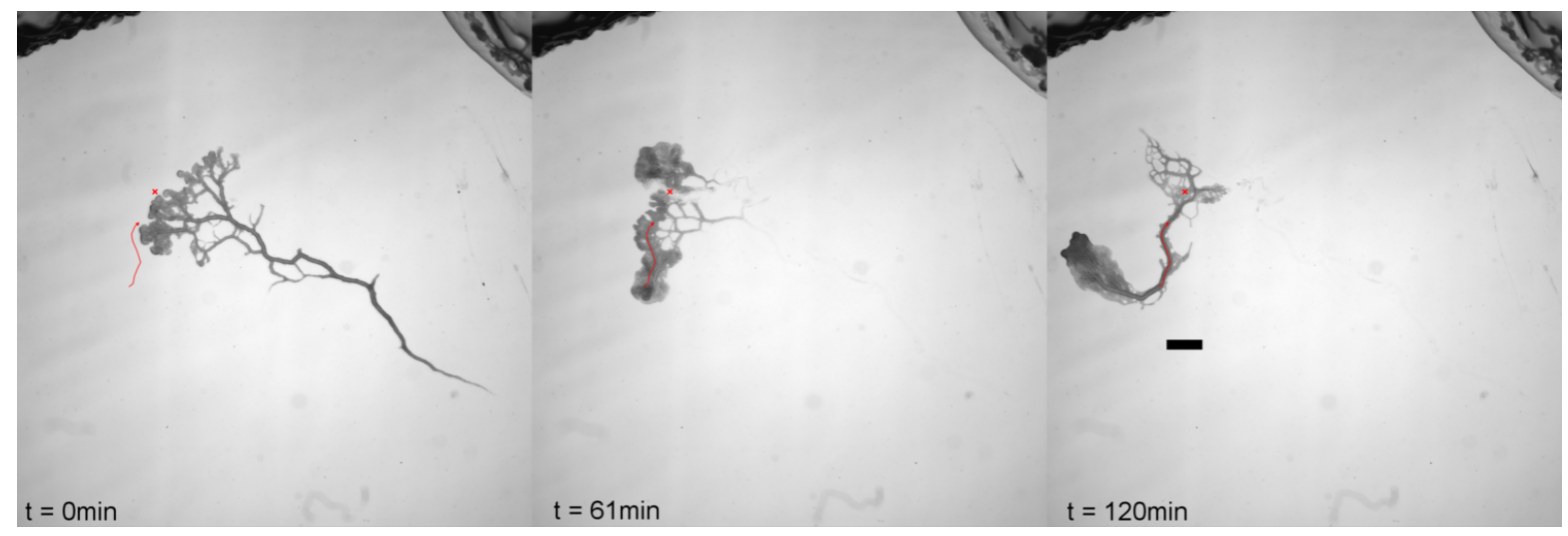

(a)

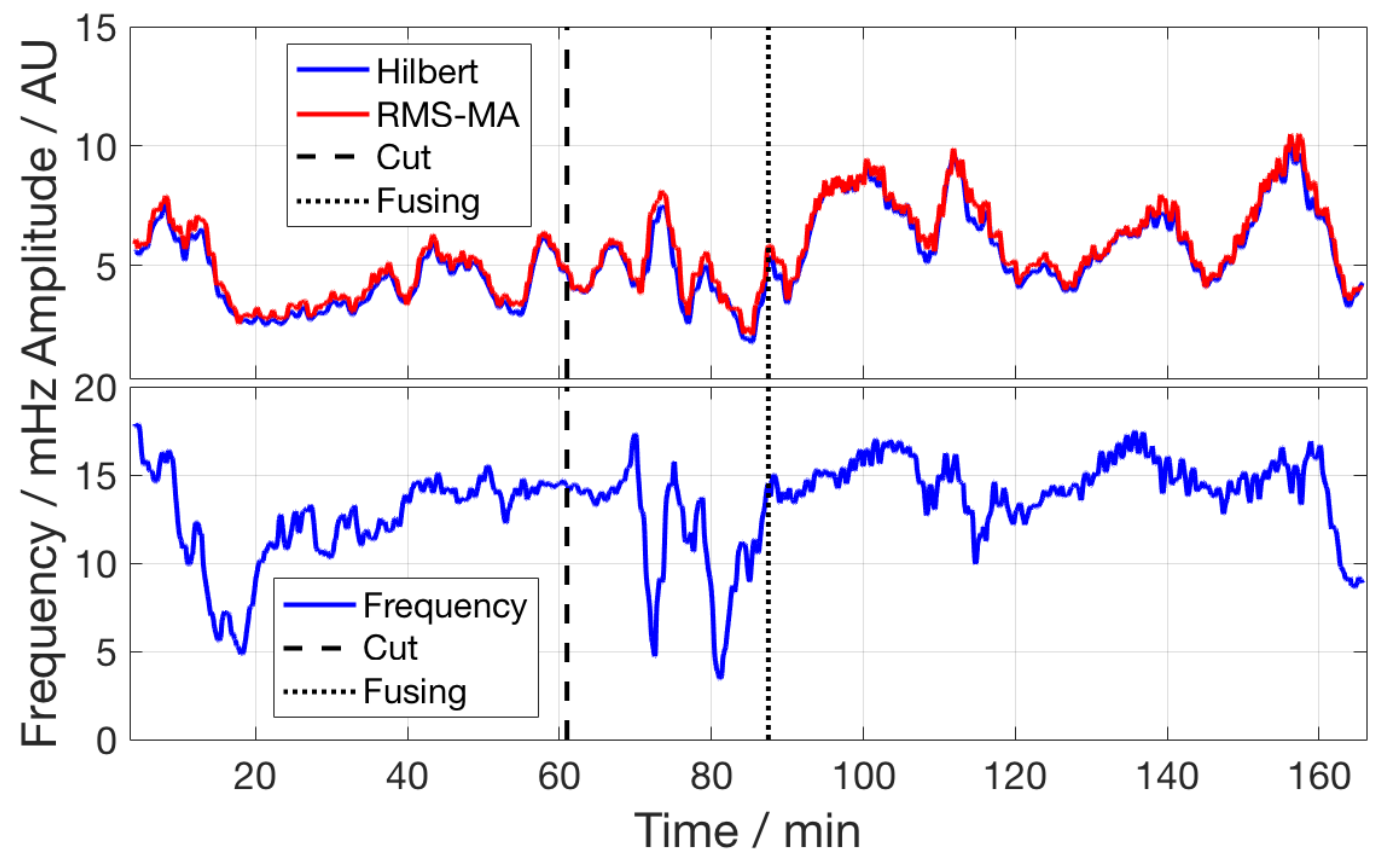

(b)

Figure E17: The plasmodium has high motility in this experiment. A cut is performed across a tube connecting two fan regions. After the cut, the material from the fan closer to the cut is moved towards the cut site and the cut parts rejoin. There is no obvious stalling. 


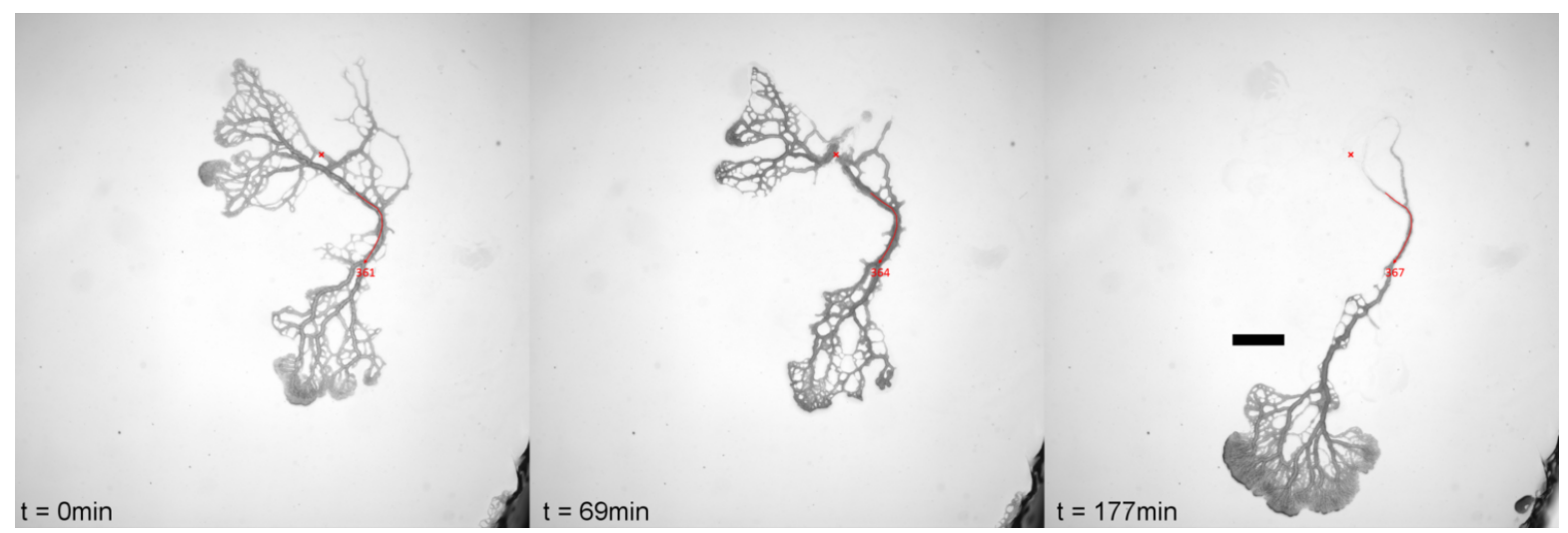

(a)

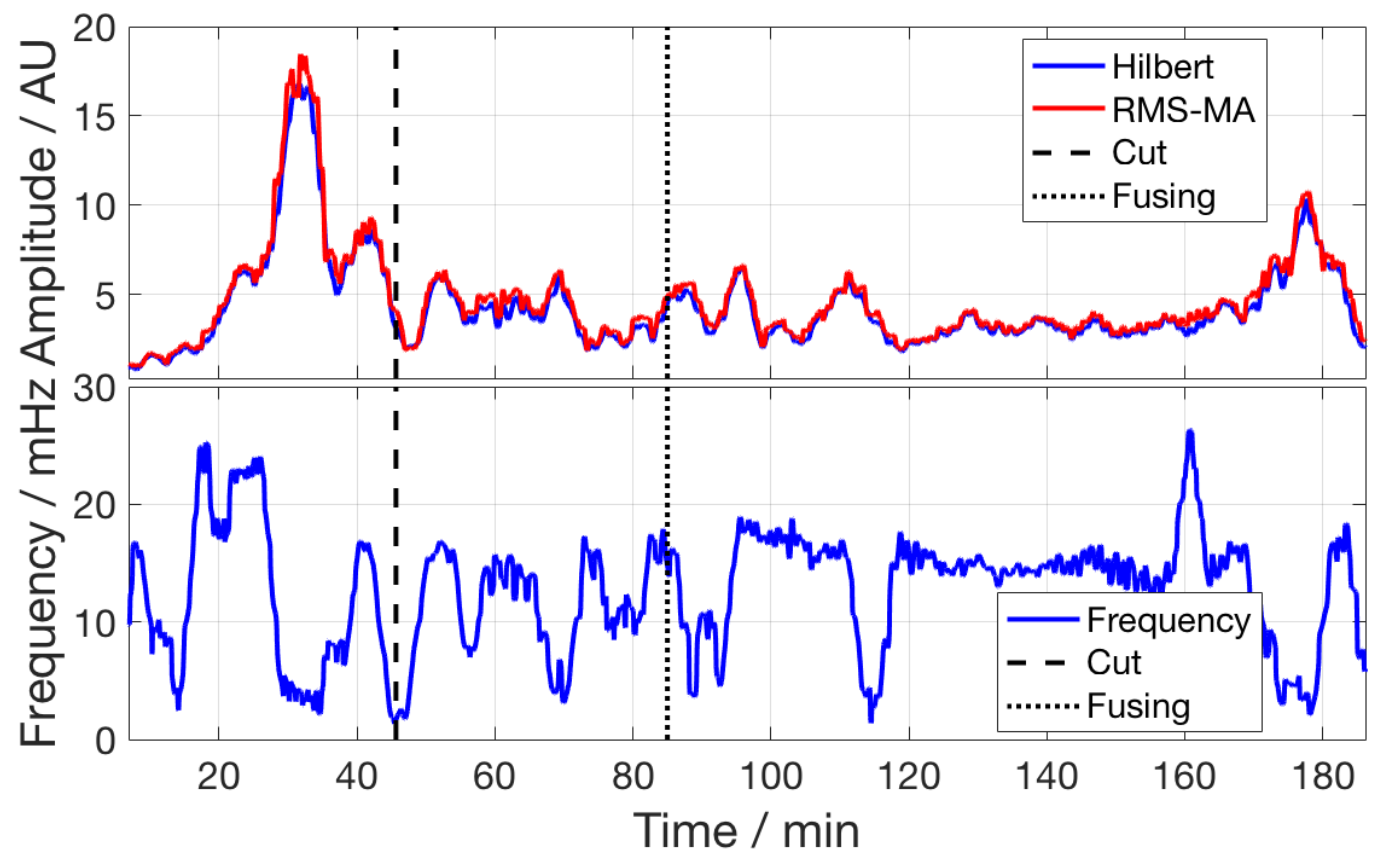

(b)

Figure E18: A cut is made across the thickest tube connecting two fan regions at the opposing ends of the organism. The organism builds a large, diffuse fan at the cut site and rejoins the cut parts. The network then moves as a whole. The variations in the frequency and amplitude of the osculations make the identification of stalling challenging. 


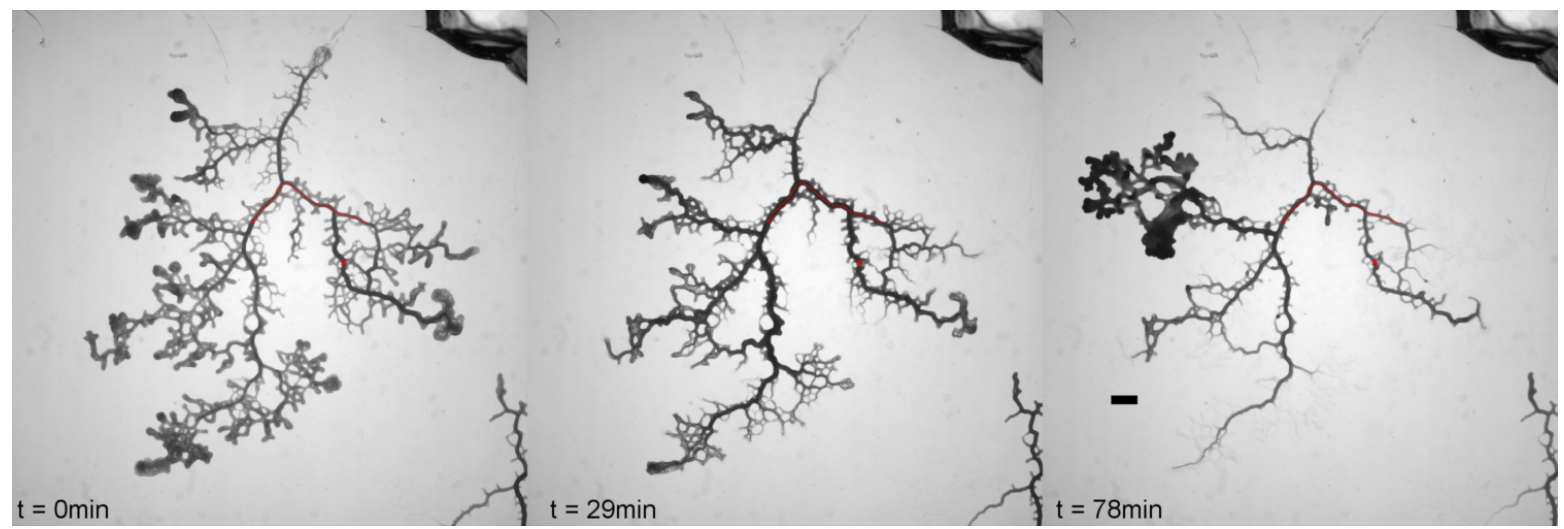

(a)

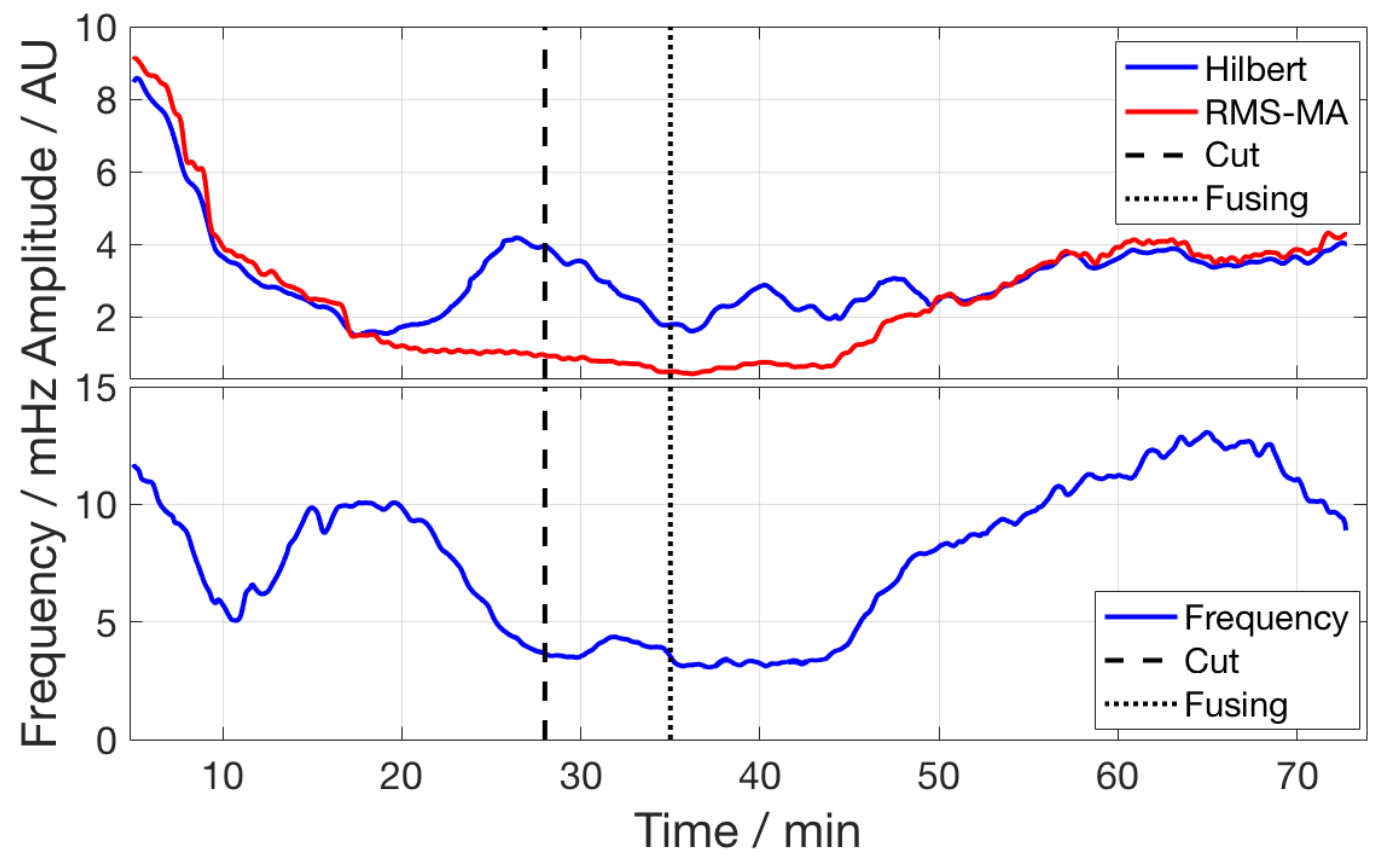

(b)

Figure E19: In this large branched network, a cut is applied across on of the thick veins, damaging it slightly. The cut parts re-join quickly and no stalling is observed. 


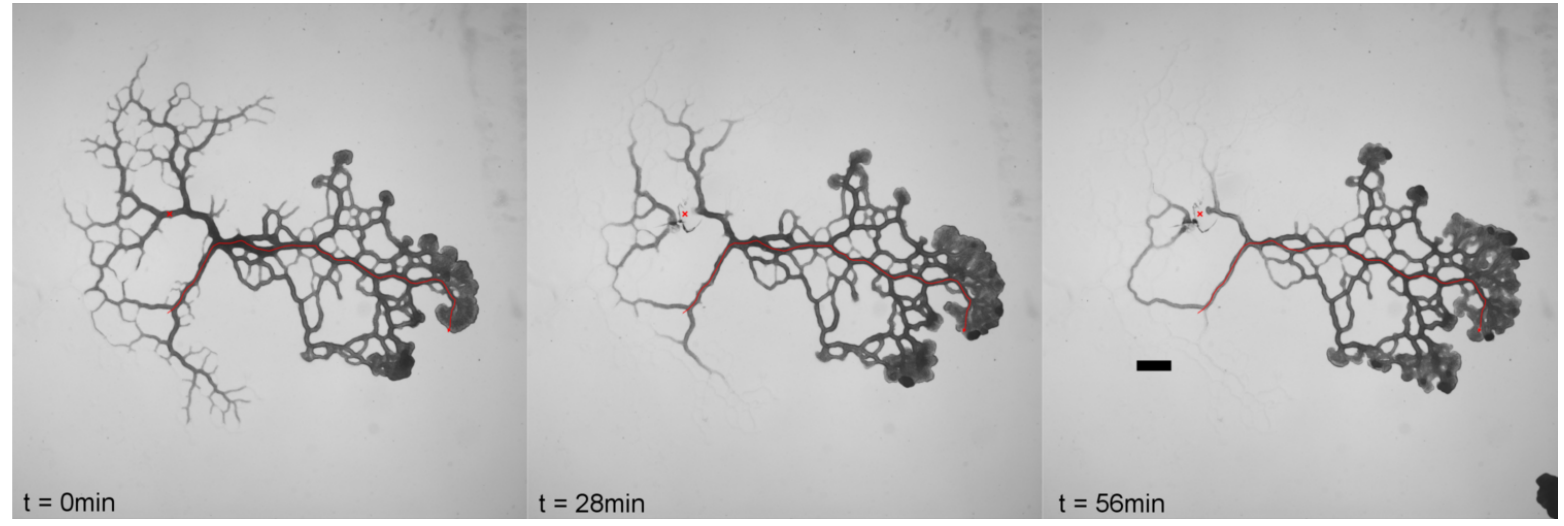

(a)

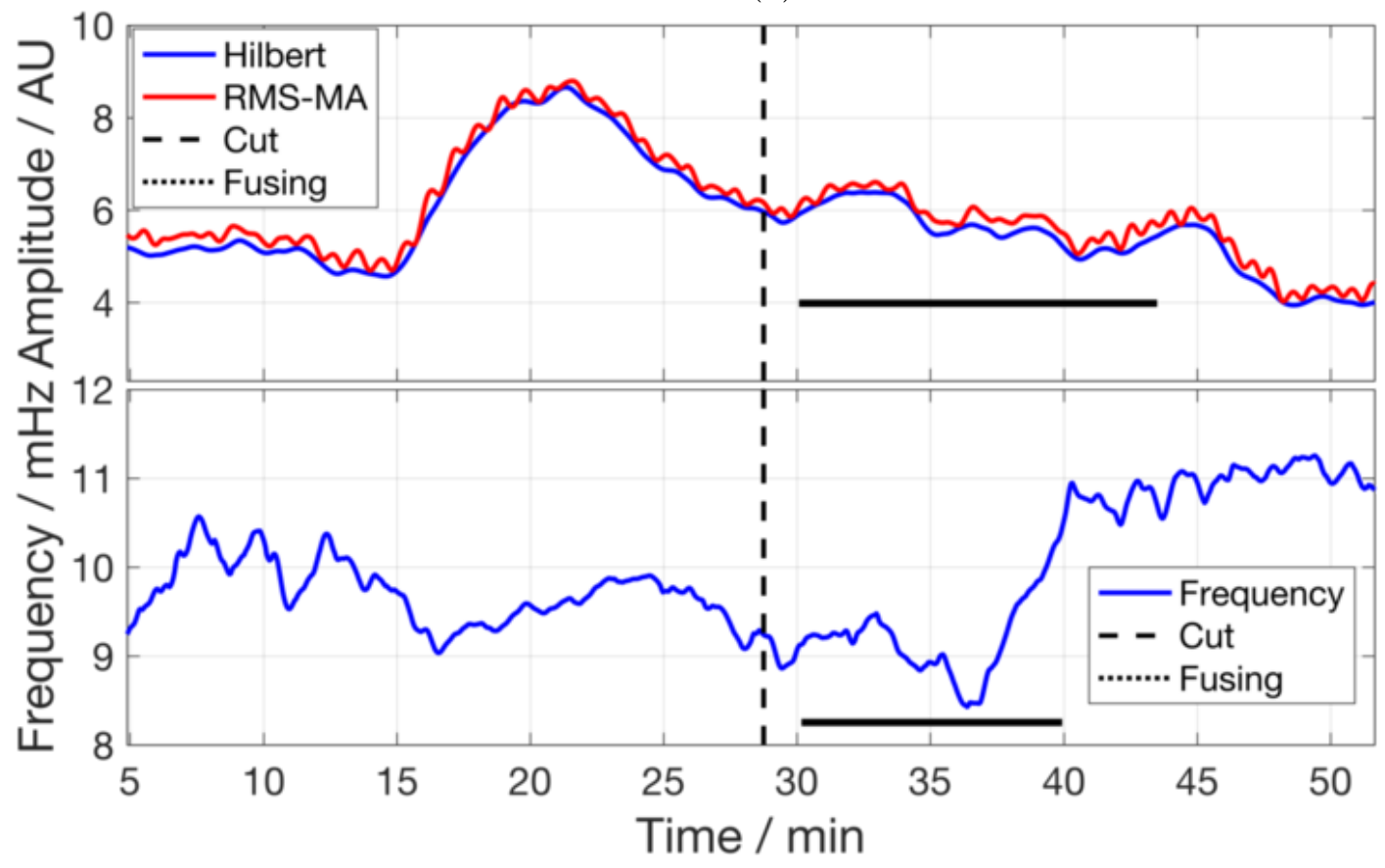

(b)

Figure E20: Here a section in the trailing part of the plasmodium is cut. Fan growth does occur, but hardly closes the gap as the neighbouring tubes are pruning to continue foraging in the distal fan region. Stalling is hardly noticeable and not evident from the amplitude graph. 


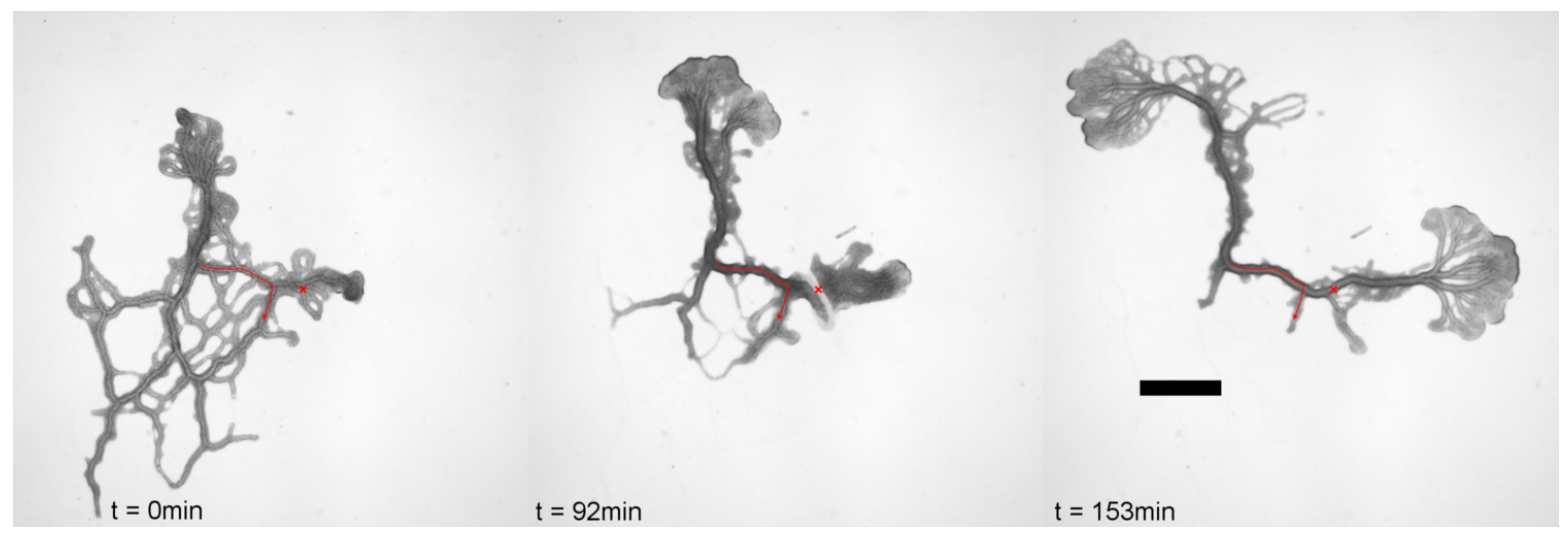

(a)

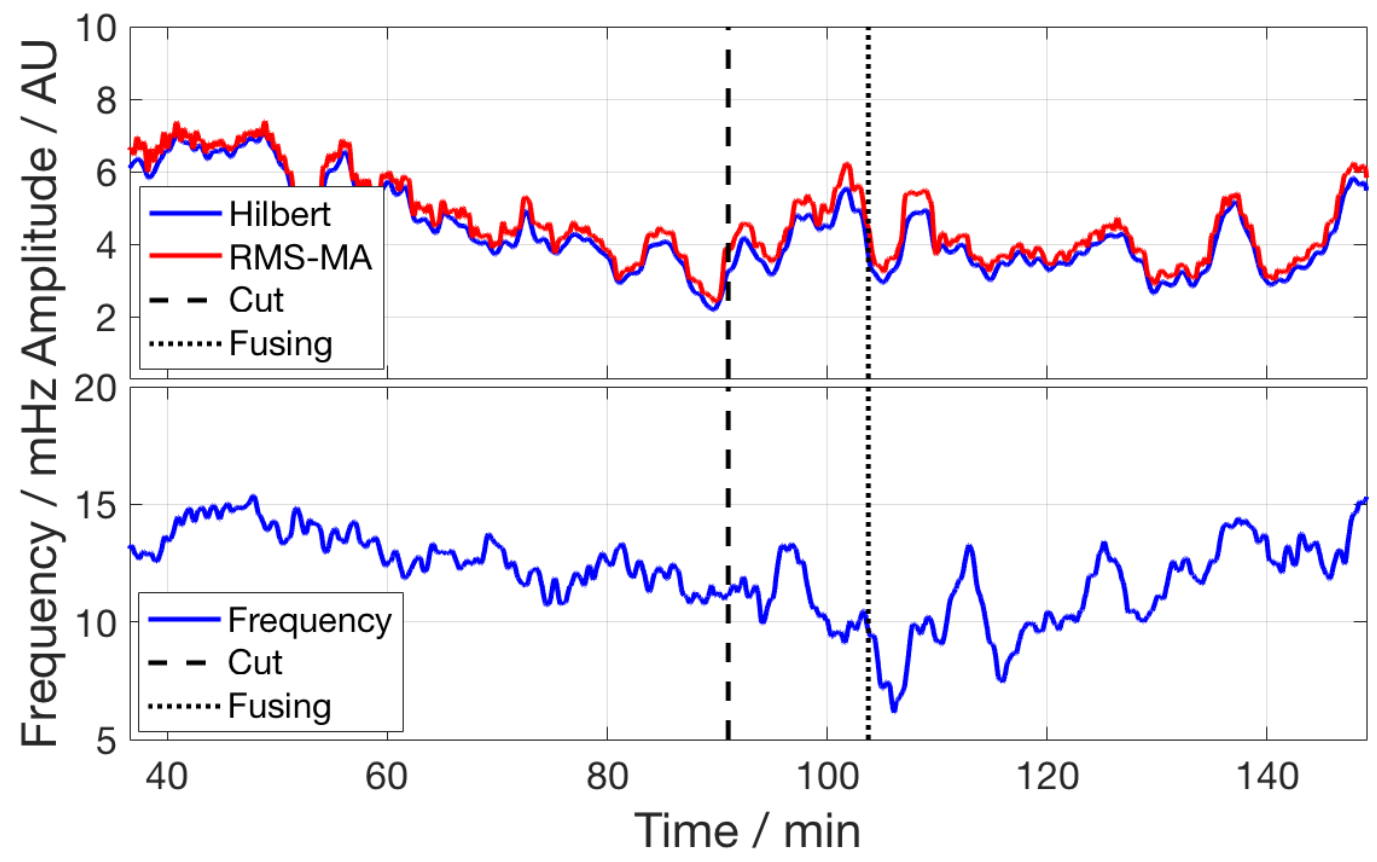

(b)

Figure E21: The small plasmodium in this experiment first undergoes a likely reaction to light, recovering into a structure made out of two fans connected by a thick tube. A cut is performed across the base of one of the fans. The tube, as well as the plasmodial sheet around it, rejoin quickly and the flow is re-established. There is no apparent stalling. 


\section{References}

[I] Achenbach, F. \& Wohlfarth-Bottermann, K. E. (I98 I). Morphogenesis and Disassembly of the Circular Plasmalemma Invagination System in Physarum polycephalum. Differentiation, I9(I-3), I79-1 88 .

[2] Achenbach, U. (1981). Synchronization and Signal Transmission in Protoplasmic Strands of Physarum. Planta, (pp. 574-583).

[3] Aldrich, H. (20 I 2). Cell biology of Physarum and Didymium VI: organisms, nucleus, and cell cycle. Elsevier.

[4] Alim, K., Amselem, G., Peaudecerf, F., Brenner, M. P., \& Pringle, A. (2013). Random network peristalsis in Physarum polycephalum organizes fluid flows across an individual. Proc. Natl. Acad. Sci., I IO(33), I3306-I 33 I I.

[5] Alim, K., Andrew, N., Pringle, A., \& Brenner, M. P. (20I7). Mechanism of signal propagation in Physarum polycephalum. Proc. Natl. Acad. Sci., I I 4(20), 5 I $36-5$ I 4 I.

[6] Avramova, Z. (20I7). The jasmonic acid-signalling and abscisic acid-signalling pathways cross talk during one, but not repeated, dehydration stress: a non-specific 'panicky' or a meaningful response? Plant Cell Environ., 40(9), I704-1710.

[7] Bailey, J., Anderson, R. W., \& Dee, J. ( I990). Cellular events during sexual development from amoeba to plasmodium in the slime mould Physarum polycephalum. $J$. Gen. Microbiol., I 36(4), 739-75 I.

[8] Baluška, F. \& Reber, A. (2019). Sentience and Consciousness in Single Cells: How the First Minds Emerged in Unicellular Species. BioEssays, 4I(3), I 800229.

[9] Banerjee, S., Gardel, M. L., \& Schwarz, U. S. (2020). The Actin Cytoskeleton as an Active Adaptive Material. Annu. Rev. Condens. Matter Phys., I I (I), 42 I-439. 
[ı。] Bäuerle, F. K., Karpitschka, S., \& Alim, K. (2020). Living System Adapts Harmonics of Peristaltic Wave for Cost-Efficient Optimization of Pumping Performance. Phys. Rev. Lett., I 24(9), 098 I02.

[I I] Bäuerle, F. K., Kramar, M., \& Alim, K. (20I7). Spatial mapping reveals multi-step pattern of wound healing in Physarum polycephalum. J. Phys. D. Appl. Phys., 5o(43).

[12] Beekman, M. \& Latty, T. (2015). Brainless but Multi-Headed: Decision Making by the Acellular Slime Mould Physarum polycephalum. J. Mol. Biol., 427(23), 37343743 .

[13] Bellon, L., Ciliberto, S., \& Laroche, C. (2000). Memory in the aging of a polymer glass. Europhys. Lett., 5 I (5), 55 I-556.

[I4] Berman, G. J., Bialek, W., \& Shaevitz, J. W. (20I6). Predictability and hierarchy in Drosophila behavior. Proc. Natl. Acad. Sci. U.S. A., I I 3 (42), I I 943 - I 1948.

[I s] Berman, G. J., Choi, D. M., Bialek, W., \& Shaevitz, J. W. (20 I 4). Mapping the stereotyped behaviour of freely moving fruit flies. J. R. Soc. Interface, I I (99).

[16] Berridge, M. J., Lipp, P., \& Bootman, M. D. (2000). The versatility and universality of calcium signalling. Nat. Rev. Mol. Cell Biol., I( I), I I-2 I.

[17] Boisseau, R. P., Vogel, D., Dussutour, A., \& Dussutour, A. (2016). Habituation in non-neural organisms : evidence from slime moulds.

[I8] Boulware, M. J. \& Marchant, J. S. (2008). Timing in Cellular Ca2+ Signaling. Curr. Biol., I 8( I7), 769-776.

[ı9] Brini, M. \& Carafoli, E. (2000). Calcium signalling: A historical account, recent developments and future perspectives. Cell. Mol. Life Sci., 57(3), 354-370.

[20] Brunet, T. \& Arendt, D. (2016). From damage response to action potentials: Early evolution of neural and contractile modules in stem eukaryotes. Philos. Trans. R. Soc. B Biol. Sci., 37 I ( 1685$)$.

[2 I] Bykov, A. V., Priezzhev, A. V., Lauri, J., \& Myllylä, R. (2009). Doppler OCT imaging of cytoplasm shuttle flow in Physarum polycephalum. J. Biophotonics, 2(8-9), 540547 .

[22] Carafoli, E. (2002). Calcium signaling: A tale for all seasons. Proc. Natl. Acad. Sci. U. S. A., 99(3), I I I 5-I I 22. 
[23] Carafoli, E. \& Krebs, J. (2016). Why calcium? How calcium became the best communicator. J. Biol. Chem., 29I(40), 20849-20857.

[24] Casadesús, J. \& D’Ari, R. (2002). Memory in bacteria and phage. BioEssays, 24(6), 5I2-5I 8 .

[25] Catterall, W. A., Leal, K., \& Nanou, E. (2013). Calcium channels and short-term synaptic plasticity. J. Biol. Chem., 288( I 5), 10742-10749.

[26] Chen, Q., Jiang, L., Li, C., Hu, D., wen Bu, J., Cai, D., \& lin Du, J. (2012). Haemodynamics-Driven Developmental Pruning of Brain Vasculature in Zebrafish. PLoS Biol., , п(8).

[27] Chen, X., Randi, F., Leifer, A. M., \& Bialek, W. (2019). Searching for collective behavior in a small brain. Phys. Rev. E, $99(5), \mathrm{I}-\mathrm{I} 3$.

[28] Cielawska, M. \& Koodziejczyk, J. (1984). The influence of dissection and of the contact with glass on the contractile activity of plasmodial strands. Cell Biol. Int. Rep., $8($ Iо), 8 I $3-82$ I.

[29] Claessen, D., Rozen, D. E., Kuipers, O. P., Søgaard-Andersen, L., \& Van Wezel, G. P. (20I4). Bacterial solutions to multicellularity: A tale of biofilms, filaments and fruiting bodies. Nat. Rev. Microbiol., I 2(2), I I 5-I 24.

[30] Clapham, D. E. (2007). Calcium Signaling. Cell, I 3 I(6), 1047-1058.

[3 I] Dumont, J. P. \& Robertson, R. M. (1986). Neuronal circuits: An evolutionary perspective. Science (80-. )., $233(4766), 849-853$.

[32] Dussutour, A., Latty, T., Beekman, M., \& Simpson, S. J. (20 I0). Amoeboid organism solves complex nutritional challenges. Proc. Natl. Acad. Sci., I07( Iо), 4607 LP - 46 I I.

[33] Edgar, B. A. \& O'Farrell, P. H. (1989). Genetic control of cell division patterns in the Drosophila embryo. Cell, 57(I), I77-1 87.

[34] Eisenstein, E. M., Eisenstein, D., \& Smith, J. C. (200I). The Evolutionary Significance of Habituation and Sensitization Across Phylogeny: A Behavioral Homeostasis Model. Integr. Physiol. Behav. Sci., 36(4), 25 I-265.

[35] Erickson, H. P. (2007). Evolution of the cytoskeleton. BioEssays, 29(7), 668-677.

[36] Ettienne, E. (1972). Subcellular localization of calcium repositories in plasmodia of the acellular slime mold Physarum polycephalum. J. Cell Biol., 54(I), I79-I84. 
[37] Farzadfard, F. \& Lu, T. K. (20I4). Genomically encoded analog memory with precise in vivo dna writing in living cell populations. Science (80-. )., 346 (62 I I).

[38] Feng, Z., Okada, S., Cai, G., Zhou, B., \& Bi, E. (2015). Myosin-II heavy chain and formin mediate the targeting of myosin essential light chain to the division site before and during cytokinesis. Mol. Biol. Cell, 26(7), I 2 I I-I 224.

[39] Fessel, A., Oettmeier, C., Wechsler, K., \& Döbereiner, H.-G. (2018). Indentation analysis of active viscoelastic microplasmodia of P. polycephalum. J. Phys. D. Appl. Phys., 5 I(2), 024005 .

[40] Fiore-Donno, A. M., Nikolaev, S. I., Nelson, M., Pawlowski, J., Cavalier-Smith, T., \& Baldauf, S. L. (2010). Deep Phylogeny and Evolution of Slime Moulds (Mycetozoa). Protist, I6I(I), 55-70.

[4I] Foxman, E. F., Kunkel, E. J., \& Butcher, E. C. (1 999). Integrating conflicting chemotactic signals: The role of memory in leukocyte navigation. J. Cell Biol., I 47(3), 577587.

[42] Gao, C., Liu, C., Schenz, D., Li, X., Zhang, Z., Jusup, M., Wang, Z., Beekman, M., \& Nakagaki, T. (2018). Does being multi-headed make you better at solving problems? A survey of Physarum-based models and computations. Phys. Life Rev., (ii), I-26.

[43] Glöckner, G., Golderer, G., Werner-Felmayer, G., Meyer, S., \& Marwan, W. (2008). A first glimpse at the transcriptome of Physarum polycephalum. BMC Genomics, 9, I-I I.

[44] Grigoriev, P. A., Matveeva, N. B., \& Teplov, V. A. (2016). The autowave electromechanical activity of the Physarum polycephalum plasmodium. Biophys. (Russian Fed., $6 \mathrm{I}(5), 748-754$.

[45] Guttes, E., Guttes, S., \& Rusch, H. P. (1961). Morphological observations on growth and differentiation of Physarum polycephalum grown in pure culture. Dev. Biol., 3(5), 588-6I4.

[46] Guy, R. D., Nakagaki, T., \& Wright, G. B. (20 I I). Flow-induced channel formation in the cytoplasm of motile cells. Phys. Rev. E-Stat. Nonlinear, Soft Matter Phys., $84(\mathrm{I})$.

[47] Halling, D. B., Liebeskind, B. J., Hall, A. W., \& Aldrich, R. W. (2016). Conserved properties of individual Ca2+-binding sites in calmodulin. Proc. Natl. Acad. Sci. U. S. A., I I3(9), EI 216-Ei225. 
[48] Hanukogle, I., Tanese, N., \& Fuchs, E. (1983). Complementary DNA sequence of a human cytoplasmic actin. J. Mol. Biol., I63(4), 673-678.

[49] Hato, M., Ueda, T., Kurihara, K., \& Kobatake, Y. (2009). Phototaxis in True Slime Mold Physarum polycephalum. Cell Struct. Funct., I(3), 269-278.

[50] Hepler, P. K. (2016). The cytoskeleton and its regulation by calcium and protons. Plant Physiol., I7O(I), 3-22.

[5 I] Hernández-Oñate, M. A. \& Herrera-Estrella, A. (20 I 5). Damage response involves mechanisms conserved across plants, animals and fungi. Curr. Genet., 6I(3), 359372.

[52] Hilker, M. \& Schmülling, T. (2019). Stress priming, memory, and signalling in plants. Plant Cell Environ., 42(3), 753-761.

[53] Hill-Eubanks, D. C., Werner, M. E., Heppner, T. J., \& Nelson, M. T. (20 I I). Calcium signaling in smooth muscle. Cold Spring Harb. Perspect. Biol., 3(9), I-20.

[54] Hu, S. G., Liu, Y., Liu, Z., Chen, T. P., Wang, J. J., Yu, Q., Deng, L. J., Yin, Y., \& Hosaka, S. (201 5). Associative memory realized by a reconfigurable memristive Hopfield neural network. Nat. Commun., 6(May), I-5.

[55] Huang, C.-H. \& Iglesias, P. A. (20I4). Cell memory and adaptation in chemotaxis. Proc. Natl. Acad. Sci. U. S. A., I I I (43), I $5287-$ I 5288.

[56] Ideses, Y., Erukhimovitch, V., Brand, R., Jourdain, D., Salmeron Hernandez, J., Gabinet, U. R., Safran, S. A., Kruse, K., \& Bernheim-Groswasser, A. (201 8). Spontaneous buckling of contractile poroelastic actomyosin sheets. Nat. Commun., 9(I).

[57] Ishigami, M., Kuroda, K., \& Hatano, S. (1987). Dynamic Aspects of the Contractile System in Physarum Plasmodium. III. Cyclic Contraction-Relaxation of the Plasmodial Fragment in Accordance with the Generation-Degeneration of Cytoplasmic Actomyosin Fibrils. ros(July), 38 I-386.

[58] Ito, K., Sumpter, D., \& Nakagaki, T. (2010). Risk management in spatio-temporally varying field by true slime mold. Nonlinear Theory Its Appl. IEICE, I(I), 26-36.

[59] Iwamura, T. (1949). Correlations between protoplasmic streaming and bioelectric potential of a slime mold, physarum polycephalum. Shokubutsugaku Zasshi, 62(735736), I26-I3I. 
[60] Jackman, S. L. \& Regehr, W. G. (20I7). The Mechanisms and Functions of Synaptic Facilitation. Neuron, 94(3), 447-464.

[6I] Joanny, J. \& Prost, J. (2009). Active gels as a description of the actin-myosin cytoskeleton. HFSPJ., 3(2), 94-I04.

[62] Johansen, T., Johansen, S., \& Haugh, F. B. (1988). Nucleotide sequence of the Physarum polycephalum small subunit ribosomal RNA as inferred from the gene sequence: secondary structure and evolutionary implications. Curr. Genet., I 4(3), 265273.

[63] Julien, J.-D. \& Alim, K. (2018). Oscillatory fluid flow drives scaling of contraction wave with system size. (pp. I-6).

[64] Jutras, M. J. \& Buffalo, E. A. (2010). Synchronous neural activity and memory formation. Curr. Opin. Neurobiol., 20(2), I 50-I 55.

[65] K. Natsume, Y. Miyake, M. Yano, H. S. (1992). Development of spatio-temporal pattern of $\mathrm{Ca}^{2+}$ on the chemotactic behaviour of Physarum polycephalum. Protoplasma, I66, $55-60$.

[66] Kamiya, N., Allen, R. D., \& Yoshimoto, Y. (1988). Dynamic organization of Physarum plasmodium. Cell Motil. Cytoskeleton, Iо( I-2), I07-ı I6.

[67] Kato, T. \& Tonomura, Y. (1975). Ca2+-sensitivity of actomyosin ATPase purified from physarum polycephalum I. J. Biochem., 77(6), I I 27-I I 34.

[68] Kawamichi, H., Nakamura, A., \& Kohama, K. (2000). Calcium Inhibition of Cytoplasmic Streaming. In Calcium Mol. Basis Calcium Action Biol. Med. (pp. 22 I-244). Dordrecht: Springer Netherlands.

[69] Keim, N. C., Paulsen, J. D., Zeravcic, Z., Sastry, S., \& Nagel, S. R. (2019). Memory formation in matter. Rev. Mod. Phys., 9I(3), 35002.

[70] Kessler, D., Eisenlohr, L. C., Lathwell, M. J., Huang, J., Taylor, H. C., Godfrey, S. D., \& Spady, M. L. ( I 980). Physarum myosin light chain binds calcium. Cell Motil., I(I), $63-7 \mathrm{I}$.

[7 I] Kessler, D., Nachmias, V. T., \& Loewy, A. G. (I976). Actomyosin content of Physarum plasmodia and detection of immunological cross-reactions with myosins from related species. J. Cell Biol., 69(2), 393-406. 
[72] Kim, S., Laschi, C., \& Trimmer, B. (2013). Soft robotics: A bioinspired evolution in robotics. Trends Biotechnol., 3 I (5), 287-294.

[73] Kincaid, R. L. \& Mansour, T. E. (1978). Measurement of chemotaxis in the slime mold Physarum polycephalum. Exp. Cell Res., I I6(2), 365-375.

[74] Kinoshita, T. \& Seki, M. (20 14). Epigenetic Memory for Stress Response and Adaptation in Plants. Plant Cell Physiol., 55 (I I), I 859-1 863.

[75] Klein, S. B., Cosmides, L., Tooby, J., \& Chance, S. (2002). Decisions and the evolution of memory: Multiple systems, multiple functions. Psychol. Rev., I09(2), 306-329.

[76] Kohama, K. (2016). Calcium inhibition as an intracellular signal for actin - myosin interaction. $92,478-498$.

[77] Kohama, K. \& Kendrick-jones, J. ( 1986 ). The inhibitory $\mathrm{Ca}^{2+}$-regulation of the actinactivated Mg-ATPase activity of myosin from Physarum polycephalum plasmodia. $J$. Biochem., 99(5), I 433-1446.

[78] Kohama, K., Kobayashi, K., \& Mitani, S. (1980). Effects of Ca Ion and ADP on superprecipitation of Myosin B from Slime Mold, Physarum polycephalum. Proc. Japan Acad. Ser. B Phys. Biol. Sci., 56(9), 591-596.

[79] Kristan, W. B. (2016). Early evolution of neurons. Curr. Biol., 26(20), R949-R954.

[80] Kulawiak, D. A., Löber, J., Bär, M., \& Engel, H. (2019). Active poroelastic two-phase model for the motion of physarum microplasmodia. PLoS One, I 4(8), I-I 9.

[8I] Kumar, S. (20I8). Epigenetic memory of stress responses in plants. J. Phytochem. Biochem, 2(I), ero2.

[82] Kuo, I. Y. \& Ehrlich, B. E. (201 5). Signaling in muscle contraction. Cold Spring Harb. Perspect. Biol., $7(2)$, I - I 4 .

[83] Kuroda, H. \& Kuroda, R. ( I98 Ia). Origin of the membrane potential in plasmodial droplets of Physarum polycephalum: Evidence for an electrogenic pump. J. Gen. Physiol., 78(6), 637-655.

[84] Kuroda, H. \& Kuroda, R. ( $198 \mathrm{Ib}$ ). Origin of the membrane potential in plasmodial droplets of Physarum polycephalum: Evidence for an electrogenic pump. J. Gen. Physiol., 78(6), 637-655. 
[85] Kuroda, R. \& Kuroda, H. (1980). Calcium accumulation in vacuoles of Physarum polycephalum following starvation. J. Cell Sci., VOL.44, 75-85.

[86] Kuroda, R. \& Kuroda, H. ( 1982 ). Relation of cytoplasmic calcium to contractility in Physarum polycephalum. J. Cell Sci., 53(1982), 37-48.

[87] Kuroda, S., Takagi, S., Nakagaki, T., \& Ueda, T. (20 I 5). Allometry in Physarum plasmodium during free locomotion: size versus shape, speed and rhythm. J Exp Biol, 2 I 8 (Pt 23), 3729-3738.

[88] Kurtz, J. (2005). Specific memory within innate immune systems. Trends Immunol., 26(4), I86-192.

[89] Lan, G. \& Tu, Y. (2016). Information processing in bacteria : memory, computation , and statistical physics : a key issues review. Reports Prog. Phys., 79, 052601.

[90] Latty, T. \& Beekman, M. (2010). Food quality and the risk of light exposure affect patch-choice decisions in the slime mold Physarum polycephalum. Ecology, 9I(I), $22-27$.

[9I] Latty, T. \& Beekman, M. (20I I). Speed-accuracy trade-offs during foraging decisions in the acellular slime mould Physarum polycephalum. Proc. R. Soc. B Biol. Sci., 278(1705), 539-545.

[92] Lewis, O. L., Zhang, S., Guy, R. D., \& del Álamo, J. C. (2015). Coordination of contractility, adhesion and flow in migrating Physarum amoebae. J. R. Soc. Interface, I2(106), 20I4I359-.

[93] Lorthois, S., Cassot, F., \& Lauwers, F. (20 I I). Simulation study of brain blood flow regulation by intra-cortical arterioles in an anatomically accurate large human vascular network. Part II: Flow variations induced by global or localized modifications of arteriolar diameters. Neuroimage, 54(4), 2840-2853.

[94] Luxardi, G., Reid, B., Maillard, P., \& Zhao, M. (2014). Single cell wound generates electric current circuit and cell membrane potential variations that requires calcium influx. Integr. Biol. (United Kingdom), 6(7), 662-672.

[95] Marbach, S., Alim, K., Andrew, N., Pringle, A., \& Brenner, M. P. (2016). Pruning to Increase Taylor Dispersion in Physarum polycephalum Networks. Phys. Rev. Lett., I I $7($ I 7$)$, I -5 . 
[96] Marom, S. \& Shahaf, G. (2002). Development, learning and memory in large random networks of cortical neurons: Lessons beyond anatomy. Q. Rev. Biophys., 35(I), 6387.

[97] Matsumoto, K., Takagi, S., \& Nakagaki, T. (2008). Locomotive mechanism of Physarum plasmodia based on spatiotemporal analysis of protoplasmic streaming. Biophys. J., 94(7), 2492-2504.

[98] Matsumoto, K., Ueda, T., \& Kobatake, Y. (1988). Reversal of thermotaxis with oscillatory stimulation in the plasmodium of Physarum polycephalum. J. Theor. Biol., I 3 I (2), I 75-I82.

[99] Meyer, B., Ansorge, C., \& Nakagaki, T. (2017). The role of noise in self-organized decision making by the true slime mold Physarum polycephalum. PLoS One, I 2(3), eoI72933.

[ıоo] Miao, Y., Bhattacharya, S., Edwards, M., Cai, H., Inoue, T., Iglesias, P. A., \& Devreotes, P. N. (2017). Altering the threshold of an excitable signal transduction network changes cell migratory modes. I9(4).

[ıо ] Micali, G., Aquino, G., Richards, D. M., \& Endres, R. G. (20 I 5). Accurate Encoding and Decoding by Single Cells: Amplitude Versus Frequency Modulation. PLoS Comput. Biol., I I(6), I-2 I.

[102] Moe, A. M., Golding, A. E., \& Bement, W. M. (201 5). Cell healing: Calcium, repair and regeneration. Semin. Cell Dev. Biol., 45, i8-23.

[103] Morisawa, M. \& Steinhardt, R. A. (1982). Changes in intracellular pH of Physarum plasmodium during the cell cycle and in response to starvation. Exp. Cell Res., I 4O(2), $34 \mathrm{I}-35 \mathrm{I}$.

[1 04] Nakagaki, T., Iima, M., Ueda, T., Nishiura, Y., Saigusa, T., Tero, A., Kobayashi, R., \& Showalter, K. (2007). Minimum-risk path finding by an adaptive amoebal network. Phys. Rev. Lett., 99(6), I-4.

[105] Nakagaki, T., Yamada, H., \& Tóth, Á. (2000a). Maze-solving by an amoeboid organism. Nature, $407(6803), 470$.

[106] Nakagaki, T., Yamada, H., \& Ueda, T. (200ob). Interaction between cell shape and contraction pattern in the Physarum plasmodium. Biophys. Chem., 84(3), I95-204. 
[107] Nakamura, M., Dominguez, A. N., Decker, J. R., Hull, A. J., Verboon, J. M., \& Parkhurst, S. M. (2018). Into the breach: How cells cope with wounds. Open Biol., 8(10).

[108] Natoli, G. \& Ostuni, R. (2019). Adaptation and memory in immune responses. Nat. Immunol., 20(7), 783-792.

[109] Natsume, K., Miyake, Y., Yano, M., \& Shimizu, H. (1993). Information propagation by spatio-temporal pattern change of Ca2 + concentration throughout Physarum polycephalum with repulsive stimulation. Cell Struct. Funct., I 8, I I I - I I 5 .

[ı ıо] Neftci, E. O. \& Averbeck, B. B. (2019). Reinforcement learning in artificial and biological systems. Nat. Mach. Intell., I(3), I 33-I 43.

[ I I I] Netea, M. G., Schlitzer, A., Placek, K., Joosten, L. A., \& Schultze, J. L. (20 I 9 ). Innate and Adaptive Immune Memory: an Evolutionary Continuum in the Host's Response to Pathogens. Cell Host Microbe, 25(I), I3-26.

[ I I 2] Nezvetsky, A. R., Orlova, T. G., Petrukhin, O. V., \& Orlov, N. Y. (2019). Control of chemotaxis in Physarum polycephalum. Biol. Membr., 36(I), 72-76.

[I I 3] Nguyen, J. P., Shipley, F. B., Linder, A. N., Plummer, G. S., Liu, M., Setru, S. U., Shaevitz, J. W., \& Leifer, A. M. (20I6). Whole-brain calcium imaging with cellular resolution in freely behaving Caenorhabditis elegans. Proc. Natl. Acad. Sci. U. S. A., I I 3 (8), Eio74-EIo8 I.

[I I 4] Nikita, V., Victor, S., Vladimirov, N., \& Sourjik, V. (2009). Chemotaxis: how bacteria use memory. Biol. Chem., 390( I I), I097.

[i I s] Noble, R. \& Noble, D. (2018). Harnessing stochasticity: How do organisms make choices? Chaos, 28(Io).

[I16] Oettmeier, C., Brix, K., \& Döbereiner, H. G. (2017). Physarum polycephalum - A new take on a classic model system. J. Phys. D. Appl. Phys., 5o(4I).

[ I I7] Oettmeier, C. \& Döbereiner, H. G. (2019). A lumped parameter model of endoplasm flow in Physarum polycephalum explains migration and polarization-induced asymmetry during the onset of locomotion. PLoS One, I 4(4), I-28.

[I I8] Oettmeier, C., Lee, J., \& Döbereiner, H. G. (2018). Form follows function: Ultrastructure of different morphotypes of Physarum polycephalum. J. Phys. D. Appl. Phys., 5 I(I3). 
[I I9] Paredes, R. M., Etzler, J. C., Watts, L. T., Zheng, W., \& Lechleiter, J. D. (2008). Chemical calcium indicators. Methods, 46(3), I 43-I 5 I.

[I 20] Pershin, Y. V., La Fontaine, S., \& Di Ventra, M. (2009). Memristive model of amoeba learning. Phys. Rev. E - Stat. Nonlinear, Soft Matter Phys., 8o(2), I-6.

[I2 I] Pfeifer, R., Iida, F., \& Lungarella, M. (20I4). Cognition from the bottom up: On biological inspiration, body morphology, and soft materials. Trends Cogn. Sci., I 8(8), 404-4I3.

[122] Quian Quiroga, R. \& Panzeri, S. (2009). Extracting information from neuronal populations: Information theory and decoding approaches. Nat. Rev. Neurosci., io(3), I73-185.

[ı23] Radszuweit, M., Engel, H., \& Bär, M. (20 I). A model for oscillations and pattern formation in protoplasmic droplets of Physarum polycephalum. Eur. Phys. J. Spec. Top., I9I(I), I 59-172.

[124] Radszuweit, M., Engel, H., \& Bär, M. (20I4). An active poroelastic model for mechanochemical patterns in protoplasmic droplets of Physarum polycephalum. PLoS One, 9(6).

[125] Rall, J. A. (2019). Calcium and muscle contraction: the triumph and tragedy of Lewis Victor Heilbrunn. Adv. Physiol. Educ., 43(4), 476-485.

[i 26] Reddy, A. S. N. (200 I). Calcium: Silver bullet in signaling. Plant Sci., I60(3), 38 I404 .

[1 27] Redish, D. A. (2015). Memory and decision making. Physiol. Bebav., I76(5), I39I 48 .

[I 28] Regehr, W. G. \& Abbott, L. F. (2004). Synaptic computation. Nature, 43 I(October), $796-803$.

[r 29] Reid, C. R., Latty, T., Dussutour, A., \& Beekman, M. (2012). Slime mold uses an externalized spatial “memory” to navigate in complex environments. Proc. Natl. Acad. Sci., I09(43), I7490 LP - I7494.

[r 30] Reid, C. R., MacDonald, H., Mann, R. P., Marshall, J. A., Latty, T., \& Garnier, S. (20I6). Decision-making without a brain: How an amoeboid organism solves the two-armed bandit. J. R. Soc. Interface, I 3 ( I I9). 
[I 3 I] Richards, T. A. \& Cavalier-Smith, T. (2005). Myosin domain evolution and the primary divergence of eukaryotes. Nature, $436(7054)$, I I I 3- I I 8 .

[132] Rieu, J. P., Delanoë-Ayari, H., Takagi, S., Tanaka, Y., \& Nakagaki, T. (201 5). Periodic traction in migrating large amoeba of Physarum polycephalum. J. R. Soc. Interface, I 2 (I06).

[133] Ringer, S. (I883). A further contribution regarding the influence of the different constituents of the blood on the contraction of the heart. J. Physiol., 4(I), 29-42.

[I 34] Rodiek, B. \& Hauser, M. J. (20 I 5). Migratory behaviour of Physarum polycephalum microplasmodia. Eur. Phys. J. Spec. Top., 224(7), I I99-I 2 I 4.

[135] Rose, J. K. (200I). Analyses of Habituation in Caenorhabditis elegans. Learn. Mem., $8(2), 63-69$.

[136] Sachsenmaier, W., Remy, U., \& Plattner-Schobel, R. (1972). Initiation of synchronous mitosis in Physarum polycephalum. A model of the control of cell division in eukariots. Exp. Cell Res., 73(I), 4I-48.

[1 37] Sanders, D., Brownlee, C., \& Harper, J. (1 999). Communicating with calcium. Plant Cell, I I (4), 69 I-706.

[138] Sato, M., Wong, T. Z., \& Allen, R. D. (1983). Rheological properties of living cytoplasm: Endoplasm of Physarum plasmodium. J. Cell Biol., 97(4), I089-1097.

[139] Sauer, H. (1982). Developmental Biology of Physarum. Developmental and Cell Biology Series. Cambridge University Press.

[140] Sauer, H. W. (1986). Introduction to Physarum, (pp. I-I7). Springer New York: Boston, MA.

[I4I] Schaap, P., Barrantes, I., Minx, P., Sasaki, N., Anderson, R. W., Bénard, M., Biggar, K. K., Buchler, N. E., Bundschuh, R., Chen, X., Fronick, C., Fulton, L., Golderer, G., Jahn, N., Knoop, V., Landweber, L. F., Maric, C., Miller, D., Noegel, A. A., Peace, R., Pierron, G., Sasaki, T., Schallenberg-Rüdinger, M., Schleicher, M., Singh, R., Spaller, T., Storey, K. B., Suzuki, T., Tomlinson, C., Tyson, J. J., Warren, W. C., Werner, E. R., Werner-Felmayer, G., Wilson, R. K., Winckler, T., Gott, J. M., Glöckner, G., \& Marwan, W. (20I6). The physarum polycephalum genome reveals extensive use of prokaryotic two-component and metazoan-type tyrosine kinase signaling. Genome Biol. Evol., 8(1), го9-I25. 
[142] Schenz, D., Shima, Y., Kuroda, S., Nakagaki, T., \& Ueda, K. I. (20I7). A mathematical model for adaptive vein formation during exploratory migration of Physarum polycephalum: routing while scouting. J. Phys. D. Appl. Phys., $50(43)$.

[143] Sebé-Pedrós, A., Grau-Bové, X., Richards, T. A., \& Ruiz-Trillo, I. (2014). Evolution and classification of myosins, a paneukaryotic whole-genome approach. Genome Biol. Evol., 6(2), 290-305.

[144] Sharrock, J. \& Sun, J. C. (2020). Innate immunological memory: from plants to animals. Curr. Opin. Immunol., 62, 69-78.

[145] Sherry, D. F. \& Schacter, D. L. (1987). The Evolution of Multiple Memory Systems. Psychol. Rev., 94(4), 439-454.

[ I 46] Shirakawa, T. \& Gunji, Y. P. (2007). Emergence of morphological order in the network formation of Physarum polycephalum. Biophys. Chem., I 28(2-3), 253-260.

[ 1 47] Skoge, M., Yue, H., Erickstad, M., Bae, A., Levine, H., Groisman, A., Loomis, W. F., \& Rappel, W. J. (20 I 4). Cellular memory in eukaryotic chemotaxis. Proc. Natl. Acad. Sci. U.S. A., I I I (40), I 4448-I 4453.

[ I 48] Smedler, E. \& Uhlén, P. (20I4). Frequency decoding of calcium oscillations. Biochim. Biophys. Acta - Gen. Subj., I 840(3), 964-969.

[ I 49] Smith, D. A. \& Saldana, R. (I 992). Model of the $\mathrm{Ca}^{2+}$ oscillator for shuttle streaming in Physarum polycephalum. Biophys. J., 6I(2), 368-80.

[ I 50] Stephens, G. J., Osborneb, L. C., \& Bialek, W. (201 I). Searching for simplicity in the analysis of neurons and behavior. Proc. Natl. Acad. Sci. U.S. A., Io8(SUPPL. 3), I $5565-1557$ I.

[ I 5 I] Tachikawa, M. \& Mochizuki, A. (20 I 5 ). Nonlinearity in cytoplasm viscosity can generate an essential symmetry breaking in cellular behaviors. J. Theor. Biol., 364, 260265 .

[1 52] Takagi, S. \& Ueda, T. (2008). Emergence and transitions of dynamic patterns of thickness oscillation of the plasmodium of the true slime mold Physarum polycephalum. Phys. D Nonlinear Phenom., 237(3), 420-427.

[1 53] Takagi, S. \& Ueda, T. (2010). Annihilation and creation of rotating waves by a local light pulse in a protoplasmic droplet of the Physarum plasmodium. Phys. D Nonlinear Phenom., 239(I I), 873-878. 
[ I 54] Takahashi, A., Camacho, P., Lechleiter, J. D., \& Herman, B. (I 999). Measurement of intracellular calcium. Physiol. Rev., 79(4), ro89-I I 25.

[I 55] Takamatsu, A., Tanaka, R., Yamada, H., Nakagaki, T., Fujii, T., \& Endo, I. (200 I). Spatiotemporal symmetry in rings of coupled biological oscillators of physarum plasmodial slime mold. Phys. Rev. Lett., 87(7), 78 I O2-1-78 I O2-4.

[ I 56] Takemoto-Kimura, S., Suzuki, K., Horigane, S. I., Kamijo, S., Inoue, M., Sakamoto, M., Fujii, H., \& Bito, H. (2017). Calmodulin kinases: essential regulators in health and disease. J. Neurochem., I 4I(6), 808-8 I 8.

[ I 57] Takeuchi, T., Duszkiewicz, A. J., \& Morris, R. G. (2014). The synaptic plasticity and memory hypothesis: Encoding, storage and persistence. Philos. Trans. R. Soc. B Biol. Sci., 369(1633).

[158] Tanaka, H.-A., Kondo, Y., \& Nei, H. (2015). What do amoebae look before they leap?- an efficient mechanism before sporulation in the true slime mold Physarum polycephalum. Nonlinear Theory Its Appl. IEICE, 6(2), 275-284.

[I59] Tang, S. K. Y. \& Marshall, W. F. (2017). Self-repairing cells: How single cells heal membrane ruptures and restore lost structures. Science (80-. )., 356(6342), 1022I025.

[160] Tapia, C. C., Tyukin, I., \& Makarov, V. A. (2019). Universal principles justify the existence of concept cells.

[16I] Teplov, V.A. (2010). Cytomechanics of oscillatory contractions. Modeling the longitudinal dynamics of Physarum polycephalum protoplasmic strands. Biophysics $(O x f)$., $55(6), 987-995$.

[162] Teplov, V. A. (2017). Role of mechanics in the appearance of oscillatory instability and standing waves of the mechanochemical activity in the Physarum polycephalum plasmodium. J. Phys. D. Appl. Phys., 50(2 I).

[163] Tero, A., Nakagaki, T., Toyabe, K., Yumiki, K., \& Kobayashi, R. (2oroa). A method inspired by physarum for solving the steiner problem. IJUC, 6, I09-I 23.

[i64] Tero, A., Takagi, S., Saigusa, T., Ito, K., Bebber, D. P., Fricker, M. D., Yumiki, K., Kobayashi, R., \& Nakagaki, T. (20 I Ob). Rules for biologically inspired adaptive network design. Science, 327(5964), 439-442. 
[165] Traynor, D. (2000). Ca2+ signalling is not required for chemotaxis in Dictyostelium. EMBOJ., I9(I7), 4846-4854.

[I66] Trivedi, D., Rahn, C. D., Kier, W. M., \& Walker, I. D. (2008). Soft robotics: Biological inspiration, state of the art, and future research. Appl. Bionics Biomech., 5(3), 99-I I 7.

[167] Tsai, F. C., Kuo, G. H., Chang, S. W., \& Tsai, P. J. (20 I 5). Ca2+ signaling in cytoskeletal reorganization, cell migration, and cancer metastasis. Biomed Res. Int., 2015.

[i68] Ueda, T., Muratsugu, M., Kurihara, K., \& Kobatake, Y. (I976). Chemotaxis in Physarum polycephalum. Effects of chemicals on isometric tension of the plasmodial strand in relation to chemotactic movement. Exp. Cell Res., Ioo(2), 337-344.

[169] Vandenboom, R., Gittings, W., Smith, I. C., Grange, R. W., \& Stull, J. T. (2013). Myosin phosphorylation and force potentiation in skeletal muscle: Evidence from animal models. J. Muscle Res. Cell Motil., 34(5-6), 317-332.

[170] Walker, S. I. \& Davies, P. C. W. (20 I 2). The Algorithmic Origins of Life. (December 20I2), I-34.

[I7I] White, P. J. \& Broadley, M. R. (2003). Calcium in plants. Ann. Bot., 92(4), 487-5 I I.

[172] Wilson, D. A. \& Linster, C. (2008). Neurobiology of a Simple Memory. J. Neurophysiol., IO0(I), 2-7.

[173] Wohlfarth-Bottermann, K. E. (1974). Plasmalemma invaginations as characteristic constituents of plasmodia of Physarum polycephalum. J. Cell Sci., I6(I), 23-37.

[174] Yoshimoto, Y. \& Kamiya, N. (1978). Studies on contraction rhythm of the plasmodial strand I. Synchronization of local rhythms. Protoplasma, 95( I-2), 89-99.

[I75] Yoshiyama, S., Ishigami, M., Nakamura, A., \& Kohama, K. (20 Io). Calcium wave for cytoplasmic streaming of Physarum polycephalum. Cell Biol. Int., 34(I), 35-40.

[176] Yumura, S., Furuya, K., \& Takeuchi, I. (I 996). Intracellular free calcium responses during chemotaxis of Dictyostelium cells. J. Cell Sci., I09( I I ), 2673-2678.

[177] Zhang, S., Guy, R. D., Lasheras, J. C., \& del Álamo, J. C. (20I7). Self-organized mechano-chemical dynamics in amoeboid locomotion of Physarum fragments. J. Phys. D. Appl. Phys., 50(20), 204004. 
[178] Zhang, S., Lasheras, J. C., \& Del Alamo, J. C. (2019). Symmetry breaking transition towards directional locomotion in Physarum microplasmodia. J. Phys. D. Appl. Phys., $52(49)$.

[179] Zhang, Y., Kawamichi, H., Kohama, K., \& Nakamura, A. (2016). Calcium-mediated regulation of recombinant hybrids of full-length Physarum myosin heavy chain with Physarum/scallop myosin light chains. Acta Biochim. Biophys. Sin. (Shanghai)., 48(6), $536-543$.

[I80] Zheng, Y., Jia, R., Qian, Y., Ye, Y., \& Liu, C. (2015). Correlation between electric potential and peristaltic behavior in Physarum polycephalum. BioSystems, I 32-I 33 , I3-I9.

[i 8 I] Zhu, L., Aono, M., Kim, S.-J., \& Hara, M. (2013). Amoeba-based computing for traveling salesman problem: Long-term correlations between spatially separated individual cells of Physarum polycephalum. BioSystems, I I 2( I), I - IO. 


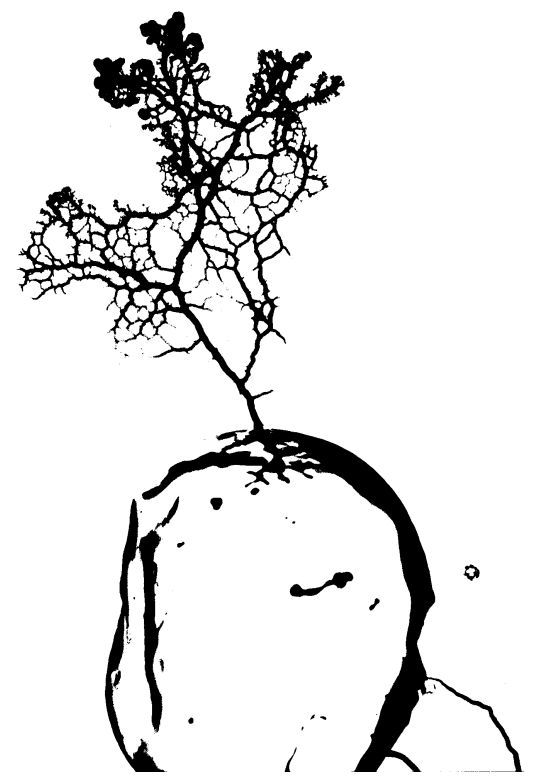

PHIS THESIS WAS TYPESET USING LTEX, originally developed by Leslie Lamport and based on Donald Knuth's TEX. The body text is set in I I point Egenolff-Berner Garamond, a revival of Claude Garamont's humanist typeface. A template that can be used to format a $\mathrm{PhD}$ thesis with this look and feel has been released under the permissive MIT (XII) license, and can be found online at github.com/suchow/Dissertate or from its author, Jordan Suchow, at suchow@post.harvard.edu. 